Prepared for the U.S. Nuclear Regulatory Commission

under an Interagency Agreement with the U.S. Department of Energy

Contract DE-AC05-76RL01830

\title{
Technical Basis for Physical Fidelity of NRC Control Room Training Simulators for Advanced Reactors
}
BS Minsk
MR Mitchell
KM Branch
BF Gore
EK Bates
DK Faris

October 2009

\section{Pacific Northwest}

NATIONAL LABORATORY

Proudly Operated by Battelle Since 1965 


\section{DISCLAIMER}

This report was prepared as an account of work sponsored by an agency of the United States Government. Neither the United States Government nor any agency thereof, nor Battelle Memorial Institute, nor any of their employees, makes any warranty, express or implied, or assumes any legal liability or responsibility for the accuracy, completeness, or usefulness of any information, apparatus, product, or process disclosed, or represents that its use would not infringe privately owned rights. Reference herein to any specific commercial product, process, or service by trade name, trademark, manufacturer, or otherwise does not necessarily constitute or imply its endorsement, recommendation, or favoring by the United States Government or any agency thereof, or Battelle Memorial Institute. The views and opinions of authors expressed herein do not necessarily state or reflect those of the United States Government or any agency thereof.

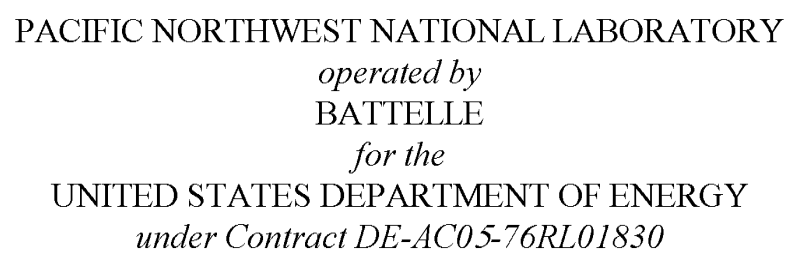

Printed in the United States of America

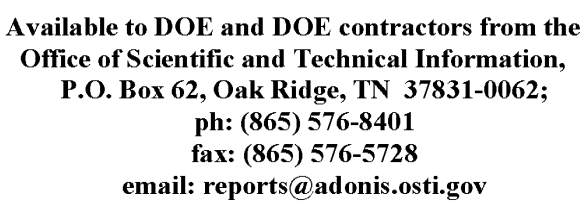

Available to the public from the National Technical Information Service, U.S. Department of Commerce, 5285 Port Royal Rd., Springfield, VA 22161 ph: (800) 553-6847 fax: $(703) 605-6900$ email: orders@ntis.fedworld.gov online ordering: http://www.ntis.gov/ordering.htm

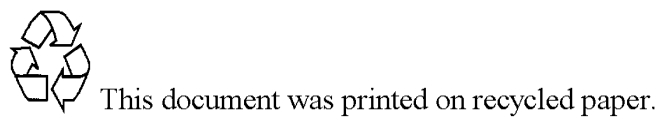




\title{
Technical Basis for Physical Fidelity of NRC Control Room Training Simulators for Advanced Reactors
}

\author{
BS Minsk MR Mitchell \\ KM Branch BF Gore \\ EK Bates DK Faris
}

October 2009

Prepared for the U.S. Nuclear Regulatory Commission under an Interagency Agreement with the U.S. Department of Energy Contract DE-AC05-76RL01830

Pacific Northwest National Laboratory

Richland, Washington 99352 


\begin{abstract}
This report provides the technical basis for physical fidelity requirements of simulator technology to train U.S. Nuclear Regulatory Commission (NRC) staff for their duties in upcoming nuclear power reactor control rooms (CRs). It assembles information from published research, expert opinion, review of next-generation CR designs, and a survey of $97 \mathrm{NRC}$ resident inspectors (RI) and operator licensing examiners (OLE) to evaluate training needs, past experience with CR simulator training, and the importance of human-system interface (HSI) systems to the tasks that comprise the CR duties of RIs and OLEs. To provide a foundation for prioritizing training and evaluating simulator physical fidelity needs, the project team developed a Task/Knowledge, Skills, Abilities (KSA) Inventory and analyzed the identified inventory items in terms of difficulty to perform, importance to safety, and frequency of performance.. The survey results provide an estimate of the degree and impact of differences in physical fidelity between the training simulator and the $\mathrm{CR} /$ simulator and assignment plants.
\end{abstract}




\section{Executive Summary}

The U.S. Nuclear Regulatory Commission's (NRC's) Technical Training Center (TCC) must develop training programs for NRC staff who will regulate advanced, or Generation III and III+, nuclear power reactors. Training in a control room (CR) simulator is an important part of the NRC training program. There is little information available on training needs or training approaches for individuals who will perform oversight tasks, and even less information on the specific needs of NRC oversight personnel. Training for jobs that involve 'hands-on' or supervisory operation/control and maintenance - aircraft pilots, medical personnel, mechanics, power plant operators, etc.- -rather than jobs that involve overseeing the work of others has been the focus of most research on simulator fidelity needs and impacts. In the absence of specific empirical data, the general assumption has often been made that, high levels of simulator fidelity are needed if simulator training is to be effective. This assumption can lead to unnecessarily expensive simulators with a level of fidelity that is not only unnecessary but detrimental to effective training. To address this lack of information, the TTC commissioned Pacific Northwest National Laboratory (PNNL) to evaluate the training needs of staff being prepared to regulate advanced reactors and the factors that affect simulator physical fidelity requirements to provide a stronger, morespecific technical basis for decisions about the fidelity of training simulators.

The fidelity of CR simulators is a multi-dimensional concept. This project's focus is on physical fidelity, specifically the level of physical fidelity needed to train both new-hire personnel, who may have no previous experience with any sort of nuclear power plant (NPP) CR, and experienced personnel, who will be assigned to advanced (Generation III and III + ) NNP reactors. The overall approach of this project was not research, in the sense of evaluating theory, but an investigation to answer the question: What does an analysis of training needs and experiences indicate about the physical fidelity requirements of a TTC training simulator for staff preparing to work at advanced NPP?

To support this investigation, the project team applied a training needs assessment approach. The

first steps of this approach are to determine what needs to be trained, rate these needs in terms of training priority, and characterize them in terms of attributes with implications for simulator fidelity needs. To establish the training needs, the project team reviewed pertinent published literature and guidance/ requirements documents; reviewed and characterized the CRs and simulators of advanced NNP reactors; assembled a series of expert panels that compiled, analyzed, and rated various aspects of the training needs; and conducted an on-line survey of current NRC resident inspectors (RIs) and operator licensing examiners (OLEs) to gather information about the level of physical fidelity in existing TTC simulator training and its impact on job performance and learning. To validate its findings, the project team also conducted focus groups and participated in workshops with experienced NRC staff. An important result of this work is the compilation and validation of an inventory of the tasks and knowledge, skills, abilities (KSA) that comprise the CR responsibilities of NRC RIs and OLEs.

An important determinant in the needs assessment and fidelity evaluation is the structure and duration of the NRC training for RIs and OLEs. The existing structure and duration is expected to apply to the training of staff for advanced reactors as well, as indicated in SECY-08-0096, "Training and Infrastructure Needs to Accomplish New Reactor Inspections and Operator Licensing," dated July 3, 2008:

“...These programs include a seven-week training regimen in either Westinghouse pressurized-water reactor or General Electric boiling-water reactor technology and use 
existing NRC simulators....The seven-week technology series includes three weeks of vendor-specific systems training, two weeks of training on transients and technical specifications, and two weeks of simulator training on normal and emergency operations [emphasis added]...."

Discussions with TTC managers, instructors, and trainees (both RIs and OLEs) revealed that the training focuses on having the trainees operate the simulator like operators. The intent is for the trainees to learn enough about the jobs so they can later evaluate the performance of operators. Furthermore, the simulator training period is only 2 weeks, so it is only long enough for teaching the concepts involved in integrating the large amount of knowledge trainees have been provided in their classroom training and for gaining an appreciation of the CR scope, feel, and the interactions between the members of the CR team. After undergoing this training, it is the responsibility of each trainee to use extensive on-the-job-training opportunities to adapt the concepts learned in the TTC training to the specific requirements of their own assigned plant CRs.

In addition, these discussions also revealed that this training policy was considered to be working well, and that the trainees accepted the need for subsequent on-the-job-training, performed it, and were satisfied with the results. Furthermore, experience indicates that their job performance was satisfactory, despite the fact the TTC simulators did not match the CRs for any of the plants to which the trainees were assigned. It is expected, and assumed for the purposes of this investigation, that this policy and practice will continue with the advent of advanced NNP reactors.

\section{Characteristics of Advanced Reactor Control Rooms and Simulators}

To gain an understanding of the attributes of the CRs and simulators for advanced reactors and how they compare with those of existing reactors, the project team reviewed available documentation, made observations, and drew upon one team member's experience in training operators at an advanced reactor in Japan. The description and comparison of the human-system interface systems (HISs) of the Advanced Boiling Water Reactor (ABWR) and the Advanced Pressurized Water Reactor (APWR) CRs and simulators provide a basis for evaluating how these differences would affect the jobs of NRC personnel responsible for regulating and overseeing these reactors. The review describes the well-recognized expansion of highly integrated HIS technology in the CR designs for the Generation III/ III+ reactors evaluated for this study. "Soft" displays and controls are used extensively in the CRs and simulators of these advanced reactors, making the HIS technology very different from that currently in use. However, the systems to be controlled and the parameters that have to be monitored to assure safe operation remain essentially the same in the advanced reactors as existing reactors. Some of the new reactor designs, such as the Advanced Passive (AP)-600 and the AP-1000, rely on passive heat removal systems, with consequent modifications of system parameters. However, details about the CR design for these plants were not available at the time this report was written. The basic designs of other advanced reactors are not different enough from current designs to require different control parameters. The jobs of the RIs and OLEs will be complicated in advanced reactor CRs because it will be harder for the RIs and OLEs to see, from a distance, what the operator is observing and what soft controls the operator is manipulating. Many control and indication systems important to safety in advanced reactors will be dedicated displays or switches that are similar to those in current use.

\footnotetext{
${ }^{1}$ Information about the passive design and its heat removal system as described by the World Nuclear Association is available at http://world-nuclear.org/info/inf08.html.
} 


\section{Task/KSA Inventory and Analysis}

To develop an inventory of operator tasks of regulatory significance, the project team drew primarily on NUREG-1122, Knowledge and Abilities Catalog for Nuclear Power Plant Operators: Pressurized Water Reactors and NUREG-1123, Knowledge and Abilities Catalog for Nuclear Power Plant Operators: Boiling Water Reactors (KA Catalogs). These NRC documents are the evolutionary outgrowth of a PNNL report, PNNL-5291, Knowledge and Abilities Required of Nuclear Power Plant Operators; Pressurized Water Reactors, which was prepared in 1984. The PNNL team determined that it needed to focus on knowledges and abilities, given that the skills required of regulators were different from those needed by operators, and that it needed to collect and aggregate the detailed operator KSA into a manageable inventory that clearly and completely spans the CR responsibilities of the operators and, from a regulatory perspective, are pertinent to NRC RI and OLE trainees. The PNNL team drew upon the stem statements used to organize the KSA catalog. The catalog provides stem statements for all systems, emergency and abnormal procedures, generic activities not specific to systems or procedures, conduct of operations, and radiological aspects of operation. In addition, the stem statements were formulated to be applicable to multiple systems and multiple reactor designs, which is an important consideration for this project. The project team used the stem statements and the KSAs listed in NUREG-1122 and NUREG-1123 to develop a preliminary task inventory of operator tasks of regulatory significance in the CR. To reduce the list into manageable size, the team then condensed it by grouping the KSA with similar intent. The goal was to have a short list that still captured all the dimensions of the CR activities of regulatory significance.

The project team then analyzed each item on the condensed list and converted the inventory items into corresponding regulatory/oversight tasks and KSAs applicable to RIs and OLEs. They drew upon the training and experience of the team members in developing content-valid licensing examinations and justifying performance evaluations of candidates, and also on their collective experience gained from working as members of NRC teams that performed special and enhanced inspections of utility operations. The team then validated the Task/KSA Inventory for each position in terms of its completeness and applicability to current and advanced reactors by comparing each inventory with documents specifying the job responsibilities of these positions, training course objectives, and review with current RI and OLE.

To better understand the training needs of RIs and OLEs, the PNNL team next convened an expert panel to develop and apply a framework of KSA types and levels to the Task/KSA Inventory items. The expert panel characterized the range and distribution of the types and levels of KSAs that RIs and OLEs would need to learn to demonstrate $\mathrm{CR}$ regulatory competence.

The team's analysis of available information about the CRs and simulators of advanced reactors indicates that, to a large extent, the Tasks/KSAs currently taught at the TTC will apply to the new reactors in the same way they apply to existing power plants. The overall licensing conditions of the Generation III/III + plants are expected to be the same as for current reactors. In addition, the regulatory goals of the RIs and OLEs are expected to be the same for the advanced reactors as for existing reactors. This analysis resulted in the compilation of a Task/KSA Inventory organized into four categories:

1) knowledge, 2) CR operations and indications (Ops), 3) operations management (Mgmt), and 4) plant license-related. 


\section{Training Priorities}

The team assembled two expert panels and performed an analysis of the Task/KSA Inventory items to rate the difficulty of performance, importance to safety, and frequency of performance of each of the Task/KSA Inventory items for each position. This analysis, referred to as the difficulty-importancefrequency (DIF) analysis, focuses on difficulty, importance to safety, and frequency in terms of performance of the task, not training. A knowledge set (K-1, K-2, K-3, K-6, and K-7) was rated in a different way; that is, as concepts required in order to successfully perform the tasks in the inventory. This analysis showed that:

- The DIF scores varied widely across the Task/KSA Inventory items and two positions

- The 15 items with the highest DIF scores are either knowledge or operations topics, the highest rated Licensing item was 16 th in rank

- Roughly half of the 20 items receiving a DIF score of 7 or more concerned system knowledge.

The same expert panels also rated each Task/KSA Inventory item in terms of the difficulty of attaining the KSAs necessary to competently perform the task (for knowledge items, the difficulty of attaining the knowledge). They also rated each Task/KSA Inventory item in terms of the necessity of using a simulator to learn how to perform the task competently (for knowledge items, to attain the knowledge). None of the Task/KSA Inventory items for RIs was rated "very difficult" to learn. For OLEs, the knowledge of plant design details and interactions between systems and components, including malfunctions of components were rated "very difficult" to learn.

The importance of using a simulator for learning varies little across the RI and OLE positions. The importance of using a simulator to train RIs and OLEs for competent job performance is high for nearly half the Task/KSA Inventory items and low for nearly one third of the items. Half of the items that rated high in importance of using a simulator for learning were among the items receiving the highest rating in terms of training priority. Simulators were rated as important for learning the Task/KSA Inventory items involving emergencies and automatic plant actuations (e.g., a reactor trip). These items require conceptual integration of large amounts of system and component operation and interaction information in real time to understand and maintain awareness of the evolving plant status. In addition, the raters also assigned this highest rating to several other OLE tasks involving responses to malfunctions, including use of normal and abnormal operating procedures and the assessment of CR command and control. The importance of using a simulator for learning addresses the desirability of using a simulator to train, but it does not address the need for any specific level of fidelity for the simulator. Training simulators need to provide enough fidelity to teach the trainees to recognize correct actions performed by operators or operator candidates in their jobs as OLEs and RIs. A few knowledge items need to be taught at the "essential to recall from memory" level, but most only need to be learned at the "understand" level. Knowledge of plant procedures was identified as particularly important, with a need to learn to execute the procedure, although not necessarily fluently. The trainees do not need to develop any specific psychomotor skills beyond auditory and visual familiarity because they do not actually manipulate controls on the job. 


\section{Characteristics and Importance of Control Room HSI Systems and System Elements}

The PNNL team drew upon the human factors and psychological literature; NUREG-0700, Rev. 2, Human-System Interface Design Review Guidelines; and the personal experience of team members with NPP CR systems and simulators to develop a framework for describing and categorizing the HSI systems and elements of current and advanced reactor CRs and simulators. The team then used this framework to define the information needed to describe and compare the CR HSI systems and simulators of the existing generation of reactor with those of the new advanced reactors. This analysis identified the following eight CR HSI systems:

- procedures

- instrumentation

- controls

- dedicated safety-related controls and displays

- displays of integrated information

- alarms/annunciators

- group-view displays

- workplace design.

An expert panel rated the importance of each of these eight CR HSI systems to each of the Task/KSA Inventory items. The results of these ratings are shown below.

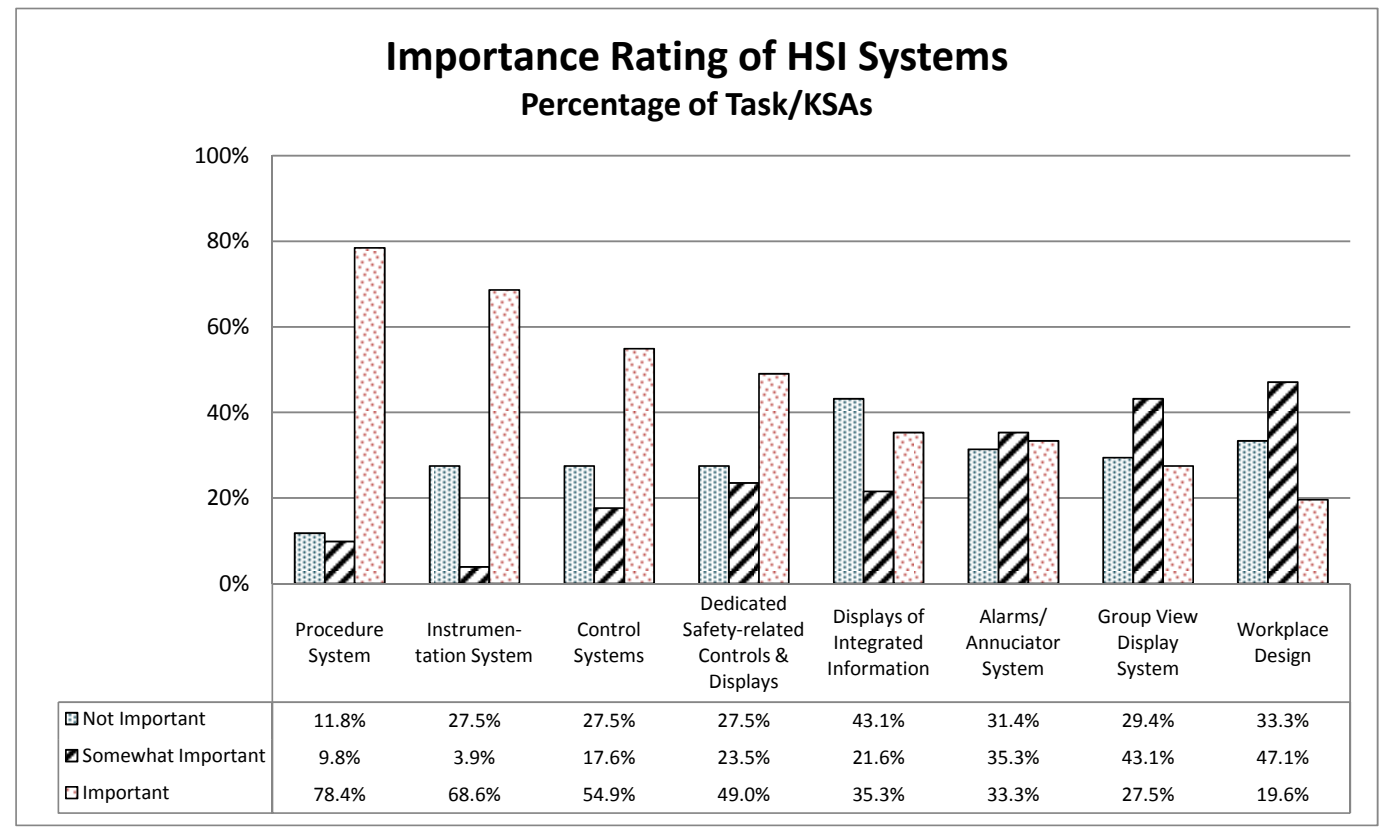


All eight HSI systems are important to the RI and OLE jobs, particularly for the operations (Ops) tasks and for one of the licensing (Lic) tasks. Procedure systems were rated important for the greatest number of Task/KSA Inventory items (78\%); workplace design was rated important for the fewest (20\%). Most of the HSI systems were rated important to tasks dealing with emergency operating procedures and abnormal operating procedures. This indicates that special attention to the physical fidelity of the HSI procedures systems of training simulators may be warranted and reinforces the finding that simulator training is particularly important in helping trainees integrate information from multiple systems.

\section{Physical Fidelity Differences and Impacts}

Seventy-four RIs and 23 OLEs who responded to an on-line rated the differences in physical fidelity between 1) a TTC simulator and 2) a corresponding CR or simulator of the same reactor vendor at a site to which they were currently assigned. These difference ratings were made for each of the eight HSI systems identified above. Respondents who reported a difference then rated the impact of that difference on job performance. As shown in the graphs below, few respondents reported "no difference" between the TTC simulator and their assigned CR or simulator. The CR environment received the highest percentage of "no difference" ratings from both RI and OLE respondents $(12.2 \%$ and $21.7 \%$, respectively), followed by procedures ( $10.8 \%$ for RIs and $8.7 \%$ for OLEs) and alarms/annunciators $(9.5 \%$ for RIs and $13.0 \%$ for OLEs). Computer displays, procedures, panels, and the CR environment received the highest "entirely different" ratings from RIs; computer displays, procedures, and alarms/annunciators were rated "entirely different" most often by OLEs. More than half of the RI respondents gave computer displays and panels a rating of " 4 " or " 5 " (very /entirely different), and more than half of the OLE respondents gave computer displays and procedures these high difference ratings.

Overall, respondents rated the impact of fidelity differences on job performance as low. Few RIs or OLEs indicated that the physical fidelity differences of any systems caused high impact (score of 5) or rated the impact as 4 , although $14.3 \%$ of the OLEs rated the impact of differences in procedures on job performance as either a 4 or 5 . Both RIs and OLEs indicated that the impact of physical fidelity differences in the CR environment were low (67\% of the RIs and $72 \%$ of the OLEs indicating no impact). These rating scores are shown in the graphs below.

Respondents who had received refresher training also rated the impact of fidelity differences on the difficulty of learning. Both RI and OLE respondents rated the impact of physical fidelity differences on difficulty of learning as low for all systems. The highest impacts were reported for procedures systems.

\section{Conclusions, Findings, and Recommendations}

The following conclusions were developed based on the analysis undertaken in this investigation. The project team also offers suggestions for consideration by NRC as training options.

1. Simulator training is imperative for nearly all tasks because:

- There is a need for integrated conceptual knowledge for nearly all tasks.

- Simulator training is effective for learning how to integrate conceptual knowledge.

- Training Suggestion. To the extent feasible, consider using simulators to train for all tasks that require integrated conceptual knowledge, using the technical basis provided in this report as a guide. 

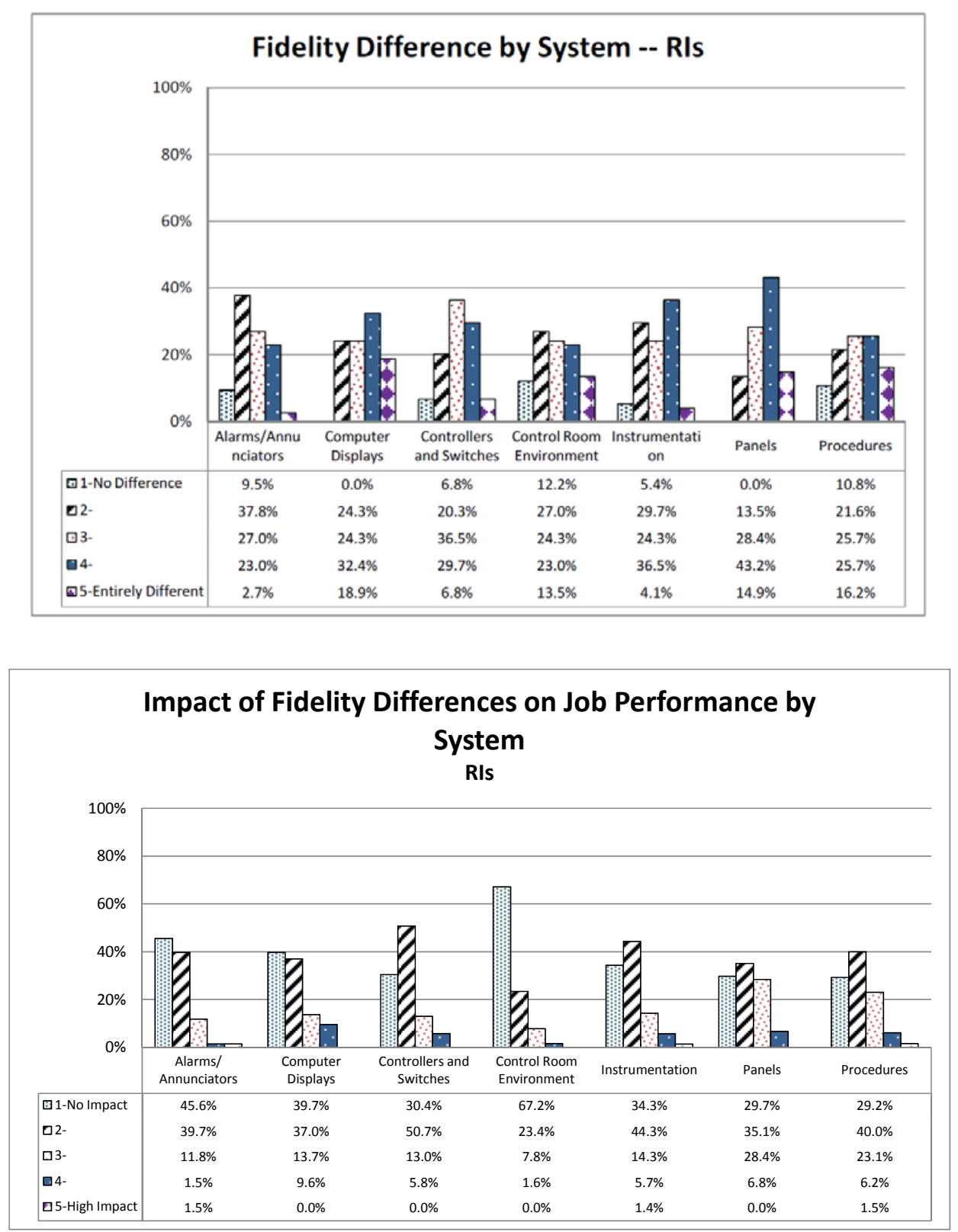

2. There is no need for simulators with full physical fidelity because:

- RIs and OLEs cannot be trained to respond automatically to tasks because in the amount of time available for simulator training is limited.

- A high level of physical fidelity can potentially interfere with learning and knowledge transfer because there probably will be a large percentage of NPP CR novices and existing trainees who will have little familiarity with new CR technology.

- A lack of simulator fidelity in current TTC training does not appear to impact the performance of existing RIs and OLEs. A lack of fidelity can be addressed through means other than simulator training. 

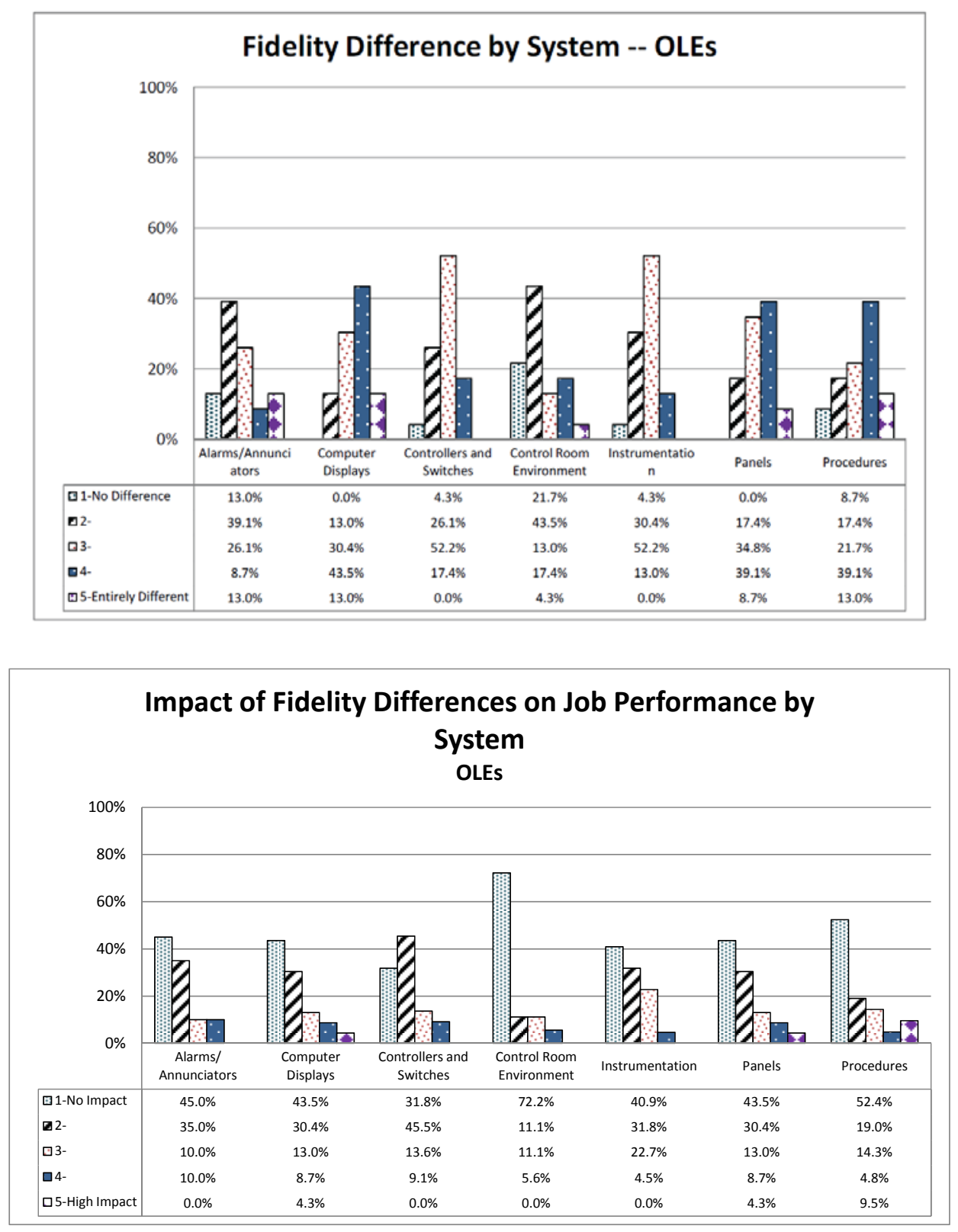

- RIs and OLEs are not responsible for operations and are not required to be trained to be able to operate an NPP. Though conventional wisdom in simulator training often points to the need for high levels of fidelity, such high fidelity levels are only required when a high degree of fluent performance of procedural and psychomotor skills is necessary. Examples are NPP operators, who must be able to react correctly and quickly during abnormal or emergency events, and Federal Aviation Administration Aviation Safety Inspectors, who must be trained to flight standards on tasks that require a high degree of psycho-motor control.

- Training Suggestion. Carefully assess whether any existing or future expectations for high levels of physical fidelity in training simulators are necessary. 
3. The full scope of CR HSI systems is required because:

- RIs and OLEs have tasks that address emergency operating procedures.

- These tasks require the full scope of CR HSI systems for competent performance.

- These tasks are among the highest importance for training.

- These tasks benefit from simulator training because they require integration of a large amount of conceptual knowledge of different types.

- Training Suggestion. Different reactors designs may require different suites of HSI systems for their CRs. Consider making sure that their simulators include the full scope of HSI systems for all new CR designs that will be operated in the United States.

- Training Suggestion. The full scope of HSI systems for a single CR can conceivably be covered in multiple simulators including multiple 'full-scope' simulators and multiple 'part-task' simulators. If multiple simulators are used for training a task for a single CR design, assess the effects of using multiple simulators on the ability to train integrated conceptual knowledge. A potential option is to use part task simulators with high fidelity for some HSI systems, where the technical basis supports this, combined with full-scope simulators that can use relatively low fidelity for HSI systems trained using higher fidelity simulators elsewhere.

- Training Suggestion. Functional HSI systems will likely have some level of integration between different HSI systems. The integration could range from having different HSI functions on the same display pages, to information flow between physically separate HSI systems, to different HSI systems being located within the same field of view. Any simulator solution considering omission of HSI integration should be evaluated for how necessary the omitted HSI integration would be for supporting training tasks effectively.

4. The potential for problems with negative transfer from simulator training exists because:

- Many staff needing simulator training will likely have no experience or familiarity with NPP CRs (F-6) or new CR HSI technology.

- Novice learners often learn to rely on perceptual cues inappropriately when there is a physical match between cues in the training context and in the performance context.

- Training Suggestion. Indicate to trainees how the training simulator is different from the CR or site-specific simulators the trainee is assigned to, with emphasis on where there is a physical match between aspects of the HSIs but the functions are different.

- Training Suggestion. To 'un-train' improper fixation on specific perceptual cues, consider training operators on the same tasks using multiple simulators with overlapping scopes, but with differences in physical fidelity. The technical basis can be used to determine the scope of HSIs needed for training on particular tasks.

5. It would be worthwhile to consider part-task, high-fidelity simulators for training interface management tasks and for supplementing TTC simulator training.

- Display interface systems are likely to be standardized within a reactor design.

- Display interface systems are likely to be the primary method for accessing plant information, managing alarms and procedures, and implementing controls. These tasks are inherent in the vast 
majority of RI and OLE tasks. CR regulatory personnel will likely need to understand interface management tasks so they can evaluate operator behavior.

- Learning interface management tasks is difficult through observation alone.

- Interface management tasks are likely to be problematic not only for operator performance, but they also may detract from NRC simulator training.

- Training Suggestion. Consider using part-task, high-fidelity simulators for training interface management tasks. Interface management tasks could conceivably be trained using simulators running on commonly available computers and operating systems and need not be hosted at a central training location.

6. Because the task and knowledge inventory for RIs and OLEs developed by this project is limited in detail and the analyses of physical fidelity needs and impacts are based on indirect measures (i.e., user and instructor reports), the investigation could not specifically determine the fidelity of the simulator HSI systems necessary for training each CR regulatory task for either currently used or advanced reactors. However, the study team is confident that the information assembled by this study is valid and will be useful for informing decisions about the relative minimum physical fidelity of the HSI systems, as well as for the importance of each of the HSI system for teaching the tasks and the training priority of the tasks.

- The current expectation is that TTC simulator training is sufficient to prepare trainees to competently train themselves onsite, and trainees can use means other than simulation training to adapt to sitespecific differences. Because of ANSI/ANS-3.5-1998 requirements, RIs and OLEs will be observing operations in full-fidelity, full-scope CRs or CR simulators onsite.

- The current expectation for TTC simulator training is that it provides the means for trainees to learn to integrate knowledge learned elsewhere and that the trainees will learn general concepts that can be applied across reactors in the same family.

- Training Suggestion. Carry out a detailed analysis of each task to be trained using a simulator to precisely determine the physical fidelity needed for each HSI system.

- Training Suggestion. Precise physical fidelity is dependent on a complete design of CR HSIs, procedures, technical specifications, etc. To date, there are no completed designs for new CRs. A detailed analysis of each CR design should be carried out to determine its physical fidelity for each HSI system.

- Training Suggestion. The goals and expectations of current TTC simulator training have evolved based on a number of factors (e.g., the variety of CR designs in operating reactors, even within the same reactor design, the availability and cost of NPP CR simulators, time constraints for simulator training, etc.). Because there is expected to be much more CR standardization within a reactor design and it may be more feasible to have simulators with higher levels of fidelity, consider if it is feasible to train the site-specific aspects of tasks, given available time and resource limitations and training priorities.

- Training Suggestion. Because precise fidelity requirements cannot currently be determined and CR HSI technology typically changes more frequently than other technology in a reactor, consider purchasing training simulator technologies that can be easily adapted to approximate the precise fidelity requirements derived from specific CR designs. 
The project team believes that the information developed and presented in this report is new, and will be useful to the NRC staff as they proceed with their analyses and decision-making concerning the training of staff for both current and next generation reactors and the acquisition of simulators to support this training. 


\section{Acknowledgements}

The PNNL project team gratefully acknowledges assistance received in conducting this research from our NRC project managers, Mark Miller and Steve Cochrum and the many NRC staff members who participated in the survey, focus group, and workshop who generated the raw data upon which this report is based. Our work was greatly enhanced by your participation. We also thank Brian Dunlop, Course Manager, Technologically Advanced Aircraft Program (Federal Aviation Administration/Flight Standards Service/Training Division/Plans and Programs Branch) for providing information about practice in the aviation sector. We also thank Oksana Elkhamri, who assisted with the first summary of the study results

and Timm Bettendorf, Nancy McGuire-Moffitt, Robert Orton, Ray Pugh, and Kenneth Erickson for rating control room human-system interface systems by importance as shown in Appendix D. Christopher Toomey provided analytical and editorial assistance in assembling the final report and Sadie Johnson assisted with formatting and graphics. 


\section{Glossary}

Auditory skill - Includes auditory memory and auditory analysis (with the same attributes as visual skill).

Basic Principles Simulator - A simulator that illustrates general concepts and demonstrates and displays the fundamental physical process of a plant.

Distributed Control System (DCS) - A DCS refers to a control system in which the controller elements are not centrally located but are distributed throughout the system with each component sub-system controlled by one or more controllers. The entire system of controllers is connected by a network for communication and monitoring.

DIF Analysis - An analysis of the difficulty of performing a task or applying knowledge, the importance of the task or knowledge to safety, and the frequency of performance or application of the task or knowledge in a job or position.

Fidelity - The degree to which one thing represents another.

Full-Scope Simulator - A simulator incorporating detailed modeling of those systems of the referenced plant with which the operator interfaces in the actual control room (CR) environment. Replica CR operating consoles are included.

Functional Fidelity - Functional fidelity refers to the similarity in perceived operation of the simulated and real situations (i.e., the simulator behaves like the real thing).

Human-System Interface (HSI) - The means of communication between a human user and a computer system, referring in particular to the use of input/output devices with supporting software. These include graphics devices, touch-sensitive devices, and voice-input devices. They have to be configured in a way that will facilitate an efficient and desirable interaction between a person and the system.

Motor skill - Movements of hand/fingers involving voluntary movement to complete a task.

Other-Than-Full-Scope Control Room Simulator (OTFSCRS) - A simulator that does not provide the same human-machine interface as does the plant to which it is referenced.

Part-Task Simulator - A simulator that incorporates detailed modeling of a referenced plant but of only some systems or portions of systems, enabling a trainee to train on only parts of a job or task.

Physical Fidelity - The simulator or simulated situation looks and feels like the real thing (i.e., the simulator looks and feels like the CR or the CR simulator).

Training Priority - A relative measure of the importance of training a task or knowledge derived from consideration of the difficulty of performing the task or applying the knowledge, the importance to safety, and the inverse of the frequency of with which the individual will be called upon to perform the task or apply the knowledge on the job for which the individual is being trained. 
Transfer - The application of knowledge skills and abilities acquired during training to the performance context that is the target of the training.

Visual Skill - Includes visual memory, which is the ability to recall visual information (including objects and scenes, temporal and spatial aspects); visual analysis, which is the ability to visually match, discriminate, or identify; and visualization, which is 'virtual' manipulation of a physical object in one's thoughts. 


\section{Acronyms}

\begin{tabular}{|c|c|}
\hline ABWR & advanced boiling water reactor \\
\hline ANS & American Nuclear Society \\
\hline ANSI & American National Standards Institute \\
\hline $\mathrm{AP}-1000$ & Advanced Passive -1000 design (Westinghouse) \\
\hline APWR & advanced pressurized water reactor \\
\hline AOP & abnormal operating procedures \\
\hline BW & Babcock and Wilcox \\
\hline $\mathrm{CE}$ & combustion engineering \\
\hline $\mathrm{CD}$ & compact disk \\
\hline CFR & Code of Federal Regulations \\
\hline COL & combined construction and operating license \\
\hline $\mathrm{CR}$ & (nuclear power plant) control room \\
\hline $\mathrm{CV}$ & containment vessel \\
\hline DCD & design control documents \\
\hline DCS & distributed control system \\
\hline DIF Analysis & difficulty-importance-frequency analysis \\
\hline DOT & U.S. Department of Transportation \\
\hline ECCS & emergency core cooling system \\
\hline EOP & emergency operating procedure \\
\hline E. Plan & emergency plan \\
\hline EPR & European Pressurized Reactor \\
\hline ESBWR & economic simplified boiling water reactor \\
\hline ESF & emergency safety function \\
\hline FAA & Federal Aviation Administration \\
\hline GAO & U.S. General Accounting Office \\
\hline GE & General Electric \\
\hline Gen III/Gen III+ & Generation Three and Three Plus Nuclear Reactor Designs/Plants \\
\hline HICR & highly integrated control room \\
\hline HSI & Human-System Interface \\
\hline HVAC & heating, ventilation, and air conditioning \\
\hline IAEA & International Atomic Energy Agency \\
\hline IMC & inspection manual chapter \\
\hline $\mathrm{I} \& \mathrm{C}$ & instrumentation and controls \\
\hline KA Catalog & Knowledge Ability Catalog \\
\hline KSA & knowledge, skills, abilities \\
\hline $\mathrm{MCC}$ & main control console \\
\hline
\end{tabular}




$\begin{array}{ll}\text { NPP } & \text { nuclear power plant } \\ \text { NRC } & \text { U.S. Nuclear Regulatory Commission } \\ \text { OCC } & \text { operator control console } \\ \text { OECD } & \text { Organisation for Economic Co-operation and Development } \\ \text { OJT } & \text { on-the-job training } \\ \text { OLE } & \text { operator licensing examiner } \\ \text { PCS } & \text { plant control system } \\ \text { PGCS } & \text { plant generation control system } \\ \text { PMCS } & \text { performance monitoring control system } \\ \text { PNNL } & \text { Pacific Northwest National Laboratory } \\ \text { PSMS } & \text { protection and safety monitoring system } \\ \text { RCIS } & \text { reactor coolant injection system } \\ \text { RCS } & \text { reactor cooling system } \\ \text { RHR } & \text { residual heat removal } \\ \text { RI } & \text { resident inspector } \\ \text { RO } & \text { reactor operator } \\ \text { RPV } & \text { reactor pressure vessel } \\ \text { SAT } & \text { systematic approach to training } \\ \text { SC } & \text { supervisor console } \\ \text { SDCV } & \text { spatially dedicated continuously visible } \\ \text { SPDS } & \text { safety parameter display system } \\ \text { SRO } & \text { senior reactor operator } \\ \text { STA } & \text { shift technical advisor } \\ \text { SS } & \text { shift supervisor } \\ \text { TS } & \text { technical specifications } \\ \text { TTC } & \text { technical training center } \\ \text { VDU } & \text { visual display unit } \\ \text { West } & \text { Westinghouse } \\ & \end{array}$




\section{Contents}

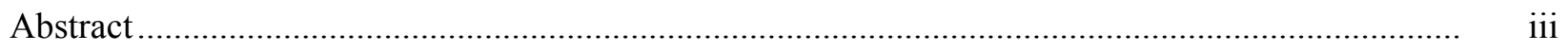

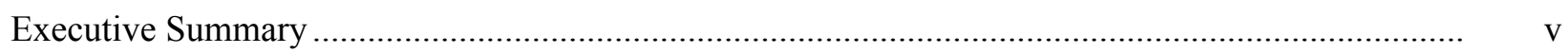

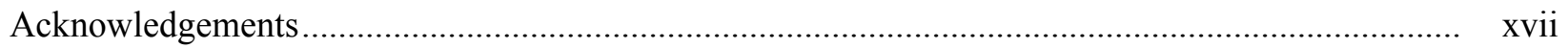

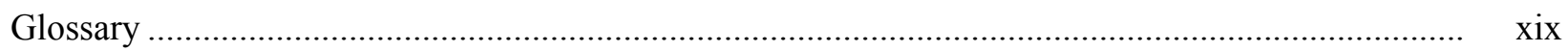

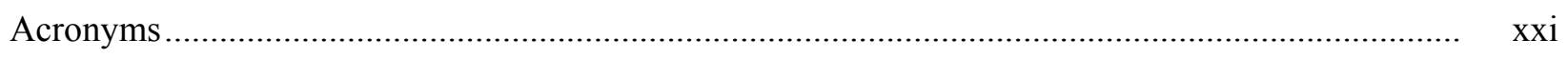

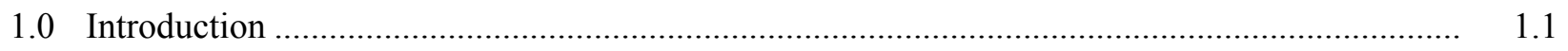

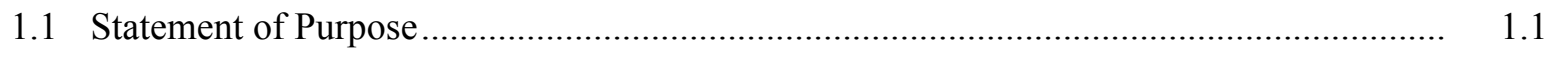

1.2 Evolution of the Project Focus and Approach............................................................. 1.2

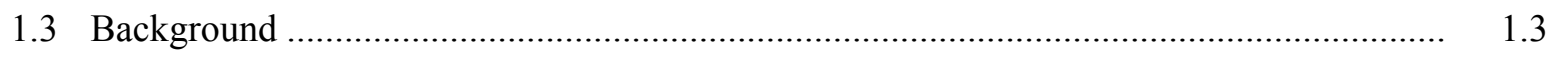

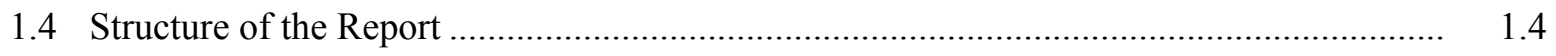

2.0 Summary of Key Literature and Practice ....................................................................... 2.1

2.1 Literature and Practice Included in the Review............................................................ 2.1

2.2 Simulator Physical Fidelity Concepts and Definitions .................................................. 2.1

2.3 Knowledge, Skills, and Abilities Frameworks ............................................................. 2.4

2.3.1 Taxonomies of the Cognitive Domain............................................................. 2.4

2.3.2 Taxonomies of Skills and Abilities .............................................................. 2.5

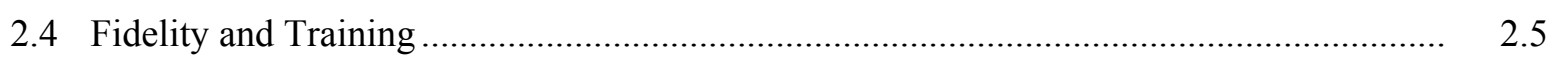

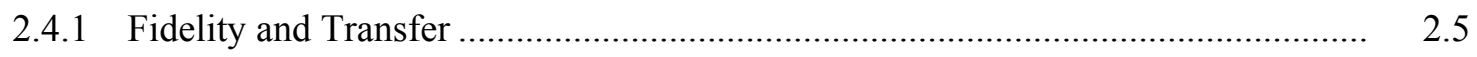

2.4.2 Simulator Use in Training ........................................................................... 2.8

2.4.3 Impacts of Simulator Physical Fidelity on Training Outcomes............................ 2.9

2.5 Representation of Knowledge and Expert vs. Novice Performance................................ 2.10

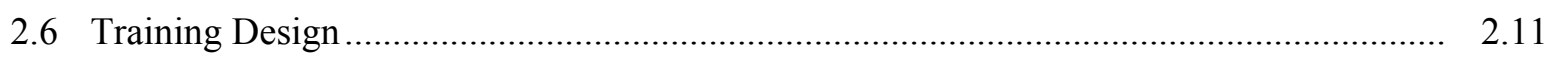

2.6.1 Systematic Approach to Training ................................................................... 2.11

2.6.2 DIF Analysis to Identify Priority Items for Training ....................................... 2.12

2.7 Experience Gained from Practice: Simulator Training of Oversight Personnel................ 2.13

2.7.1 FAA Flight Safety Inspectors ....................................................................... 2.13

2.7.2 Nuclear Power Regulatory Personnel Outside of the United States...................... 2.14

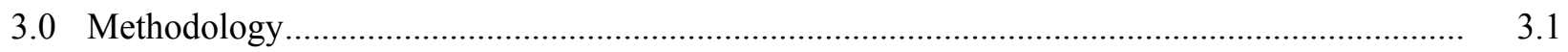

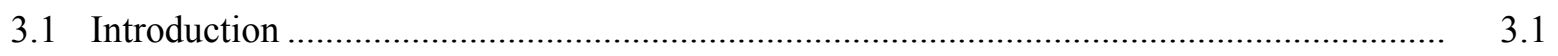

3.2 Control Room Task and KSA Inventory ...............................................................

3.2.1 Inventory of Operator Tasks of Regulatory Significance in the Control Room..... 3.2

3.2.2 Conversion of Operator Tasks/KSAs into Regulatory/Oversight Tasks and KSAs .................................................................................................. $\quad 3.4$

3.2.3 Validation of the Task/KSA Inventory for RI and OLE Positions ......................... 3.4

3.2.4 Taxonomy of the Tasks/KSA Inventory Items .................................................. 3.5

3.2.5 Discussions with NRC Staff and TTC Trainees ................................................. 3.5 
3.3 Priority of Task/KSA Items for Training .................................................................. 3.7

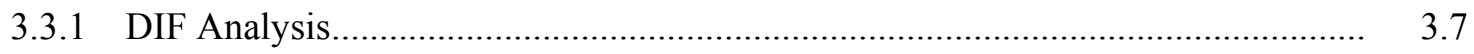

3.3.2 Difficulty of Learning and the Importance of Using a Simulator to Learn the Task/KSA Inventory Items .............................................................................. 3.8

3.4 The Type and Level of Knowledge, Skill, and Ability Needed to Perform the Task/KSA Inventory Items ............................................................................... 3.8

3.5 Role of Simulators and CR HSI Systems in Performing and Learning Task/KSA Inventory Items.

3.5.1 Dimensions of Physical Fidelity: CR HSI Systems and System Elements

3.5.2 The Importance of CR HSI Systems and Simulator Dimensions for Each Task/KSA Inventory Item ....................................................................... 3.11

3.6 Online Survey of Current RIs and OLEs.................................................................... 3.11

3.6.1 Questionnaire Design and Pretesting.......................................................... 3.12

3.6.2 Survey Distribution, Data Collection, and Analysis of Results............................ 3.12

3.7 Advanced Reactor Control Room Characteristics and Differences/Similarities with Existing Control Rooms

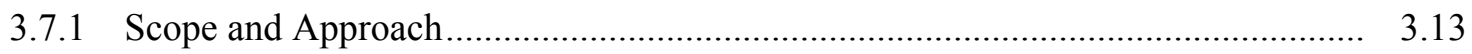

3.7.2 Attributes Evaluated and Described .............................................................. 3.13

3.8 Review of NRC Training Policies, Constraints, and Workforce Characteristics ............... 3.15

3.9 Analysis and Integration of Results......................................................................... 3.16

4.0 Advanced Reactor Control Room Characteristics ............................................................ 4.1

4.1 Overview of Major Features of Advanced Reactor Control Room Designs ..................... 4.1

4.1.1 Panel, Workstation, and Workplace Design ...................................................... 4.1

4.1.2 Hard Controllers and Switches .................................................................... 4.2

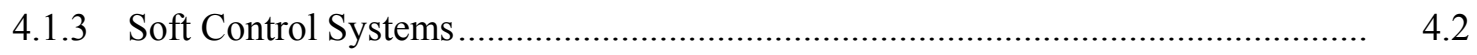

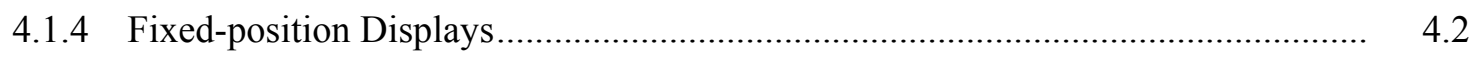

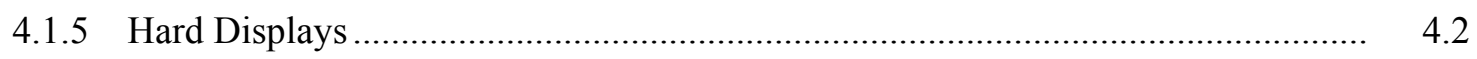

4.1.6 Single-Function (single-page) Visual Display Unit-based Displays ...................... 4.2

4.1.7 Page-Based, Single-operator Displays ........................................................... 4.2

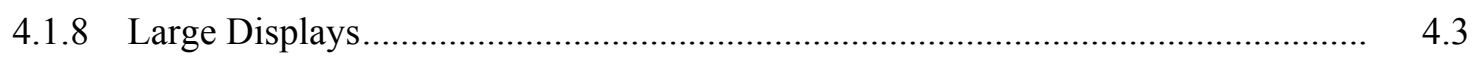

4.1.9 Computerized Operator Support Systems ….................................................... 4.3

4.1.10 Displays of Integrated Plant Information ......................................................... 4.3

4.1.11 Dedicated Safety-related Controls and Display System ..................................... 4.3

4.1.12 Alarm Display and Processing …................................................................... 4.3

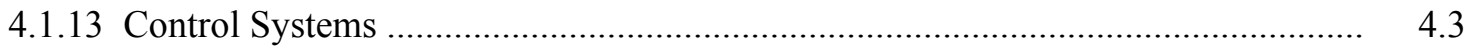

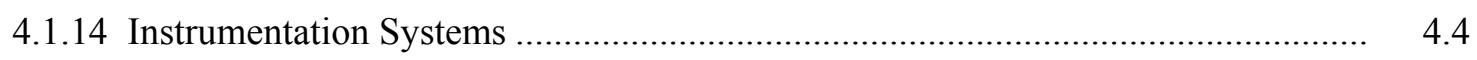

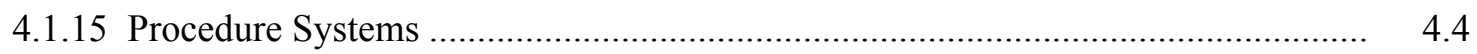

4.1.16 Group-view Display Systems ....................................................................... 4.4

4.1.17 Integration of Information Displays and Control ............................................ 4.4 
4.2 Overview of the Major Differences Between the ABWR and APWR Main Control Room HSI Technologies ................................................................................... 4.5

4.3 APWR Main Control Room .................................................................................... 4.6

4.3.1 Basic Characteristics ................................................................................. 4.6

4.3.2 Detailed Descriptions of Specific Features of the APWR ................................... 4.7

4.4 ABWR Main Control Room................................................................................... 4.11

4.4.1 Basic Characteristics of the ABWR Main Control Room .................................... 4.11

4.4.2 Detailed Descriptions of Specific Features of the ABWR .................................. 4.12

4.5 Implications for Physical Fidelity of TTC Simulators in Advanced Reactor Training ....... 4.16

5.0 RI and OLE Training Needs and Priorities..................................................................... 5.1

5.1 What RIs and OLEs Need to Know: Task/KSA Inventory .......................................... 5.1

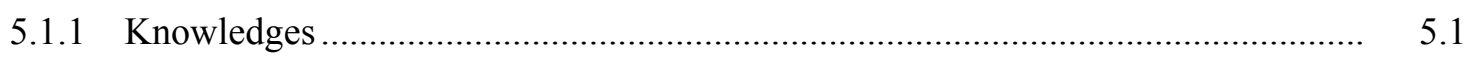

5.1.2 Control Room Operations and Indications Tasks (Ops) .................................... 5.1

5.1.3 Operations Management Tasks (Mgmt) .......................................................... 5.4

5.1.4 Plant License-Related Tasks (Lic) ............................................................... 5.4

5.2 Results of the DIF Analysis to Identify Priorities for Training RIs and OLEs ................. 5.5

5.3 Difficulty of Learning and Importance of Using a Simulator to Learn Task/KSA Inventory Items

5.4 Types and Levels of KSAs of the Task/KSA Inventory Items........................................ 5.8

5.5 Role of Simulator/HSI Systems in Training the Task/KSA Inventory Items ..................... 5.11

5.5.1 Importance of HSI Systems to Task/KSA Inventory Items................................ 5.11

5.6 Implications for Physical Fidelity of TTC Simulators in Advanced Reactor Training ....... 5.13

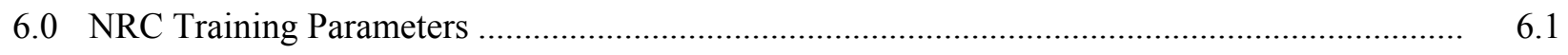

6.1 NRC Program Descriptions and Policies................................................................. 6.1

6.2 Focus Group Characterizations of Training ............................................................ 6.2

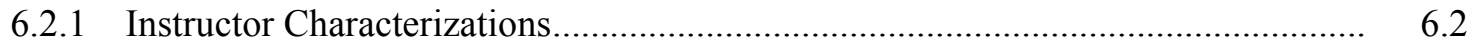

6.2.2 The Role of On-the-Job Training ..................................................................... 6.2

6.2.3 Importance of Trainee Interactions with the Plant ............................................ 6.4

6.2.4 Experience and Background of Refresher Trainees .......................................... 6.4

6.2.5 Overall Training Objectives ........................................................................ 6.4

6.3 Demographics of NRC Staff that Affect Training....................................................... 6.5

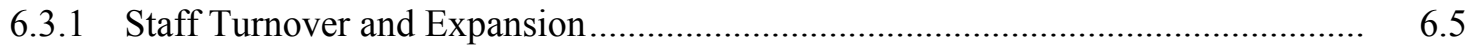

6.3.2 Years and Type of Experience of On-Line Survey Respondents .......................... 6.5

6.3.3 TTC Training Experience of On-Line Survey Respondents ................................ 6.8

6.3.4 Familiarity with New Control Room Technologies ............................................ 6.11

6.3.5 On-Line Survey Respondents' Evaluation of Current TTC Simulator Training.... 6.11

6.4 Implications for Physical Fidelity of TTC Simulators in Advanced Reactor Training ....... 6.13 
7.0 Physical Fidelity Differences between the TCC Simulator and Work Location Control

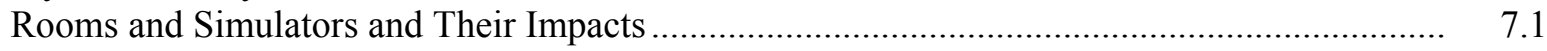

7.1 Physical Fidelity Differences of HSI Systems ..............................................................

7.2 Physical Fidelity Difference Impacts on Job Performance.................................................. 7.1

7.3 Impact of Physical Fidelity Differences on Difficulty of Learning ................................... 7.4

7.4 System by System Ratings of Differences and Impacts .................................................

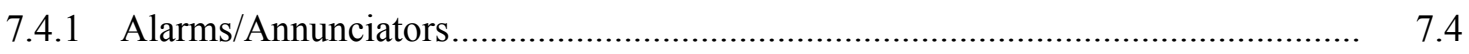

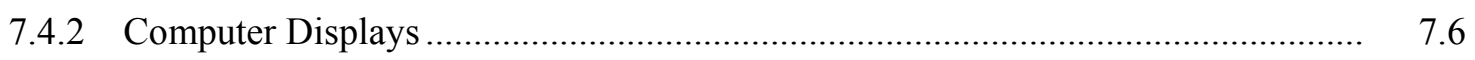

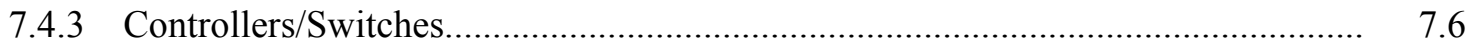

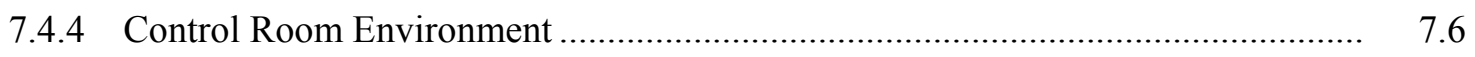

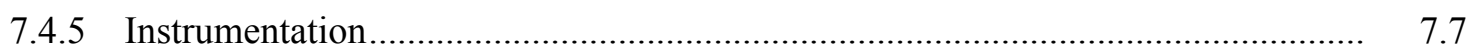

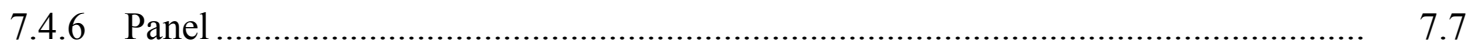

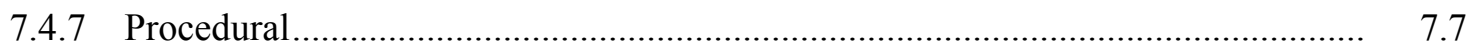

7.5 Summary of On-Line Survey Volunteered Comments about Physical Fidelity

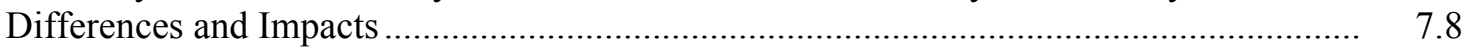

7.6 How TTC Training Addressed Physical Fidelity Differences........................................... 7.10

7.7 Implications for Physical Fidelity of TTC Simulators in Advanced Reactor Training ....... 7.11

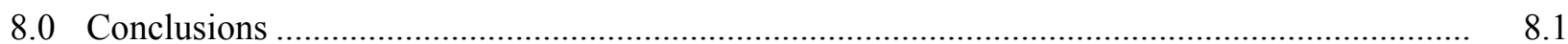

8.1 Summary of Factors Influencing Simulator Physical Fidelity Training Priorities and Needs: Current and Future............................................................................................ $\quad 8.1$

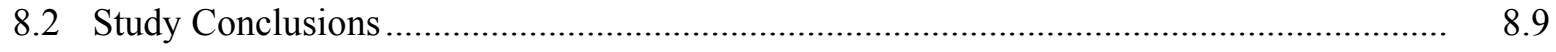

8.2.1 Summary of Findings ........................................................................ 8.10

8.3 Conclusions, Findings, and Recommendations ......................................................... 8.13

8.3.1 Suggested Framework for Applying Study Findings to Training Simulator Physical Fidelity Decisions ........................................................................ 8.16

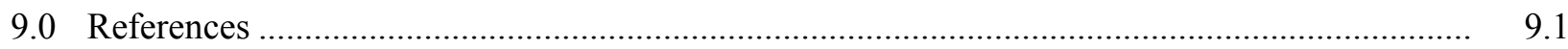

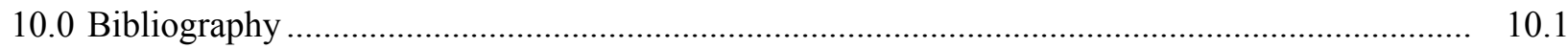

Appendix A - TTC Focus Group Discussion …........................................................................ A.1

Appendix B - DIF Analysis Rating Scales and Results ......................................................... B. 1

Appendix C - Task/KSA Types and Levels Rating............................................................... C.1

Appendix D - Reactor and Simulator CR Systems and Elements and Their Importance to Task/KSA Inventory Items............................................................................... D. 1

Appendix E - The On-Line Survey ................................................................................... E. 1

Appendix F - Draft NRC Advanced Reactor Simulator Fidelity Requirements .............................. F.1 


\section{Figures}

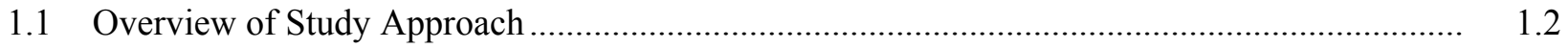

2.1 Understanding the Relevant Literature and Practice .......................................................... 2.2

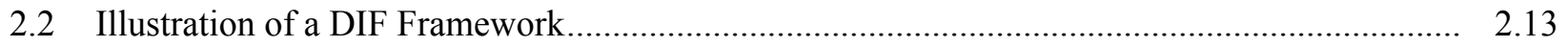

3.1 Outline of Overall Study Elements ................................................................................. 3.2

3.2 Steps in the Task Inventory Development Process................................................................. 3.3

3.3 Steps in Characterizing and Comparing Control Rooms of New Reactor Designs................... 3.14

3.4 How the Study Components Were Integrated: The Combined Training Analysis................... 3.16

4.1 Equipment Arrangement of Operator Control Console ....................................................... 4.7

4.2 Typical Layout of APWR Control Room ........................................................................ 4.11

4.3 Equipment Arrangement of Supervisor Console and STA Console........................................ 4.12

4.4 Arrangement of Large Display Panel ............................................................................ 4.12

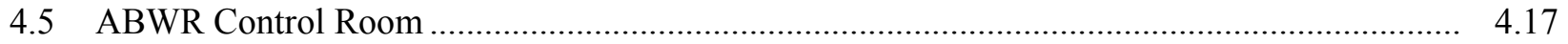

4.6 ABWR Control Room Wide Display Panel Highlighting Fixed-Display Information .............. 4.17

4.7 ABWR Control Room Wide Display Panel Highlighting Large Variable Display.................... 4.18

5.1 Percentage of Tasks/KSAs Inventory Items for Which Each HSI Systems is Important............ 5.12

5.2 Number of Tasks/KSAs Inventory Items for Which Each HSI System is Important................ 5.12

5.3 Number of CR HSI Systems Rated of High, Medium, and Low Importance to Tasks/KSA

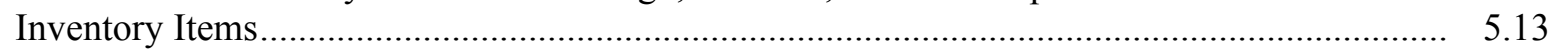

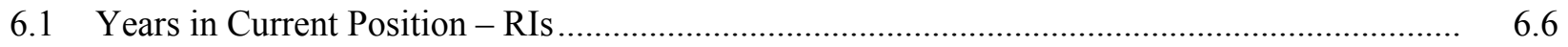

6.2 Years in Current Position - OLEs .................................................................................. 6.6

6.3 Previous Experience - All Respondents, RIs and OLEs ................................................... 6.7

6.4 Average Years of Experience in Different Positions - RIs and OLEs ..................................... 6.7

6.5 Types of Reactors of Current Assignment and Classroom and Simulator Training - RIs .......... $\quad 6.8$

6.6 Types of Reactors of Current Assignment and Classroom and Simulator Training - OLEs ...... $\quad 6.9$

6.7 Proportion of RIs and OLEs Who Have Had TTC Refresher Training .................................... 6.9

6.8 Number of Simulator Refresher Courses by Reactor Type - RIs and OLEs ........................... 6.10

6.9 Years Since Last TTC Simulator Training - RIs and OLEs................................................. 6.10

6.10 Familiarity with New Technologies - RIs and OLEs..................................................... 6.11

6.11 Evaluation of Simulator Training Effectiveness - RIs and OLEs ......................................... 6.12

7.1 Fidelity Difference Ratings for All Systems: All Respondents .............................................. 7.2

7.2 Fidelity Difference Ratings for All Systems: RIs ............................................................. 7.2

7.3 Fidelity Difference Ratings for All Systems: OLEs …........................................................

7.4 Fidelity Difference Impacts on Job Performance by System - RIs ........................................

7.5 Fidelity Difference Impacts on Performance by System - OLEs.............................................

7.6 Fidelity Difference Impacts on Difficulty of Learning by System - RIs ................................. 7.5

7.7 Fidelity Difference Impacts on Difficulty of Learning by System - OLEs.............................. 7.5

7.8 How Training Addressed Fidelity Differences - RIs and OLEs ......................................... 7.10 
8.1 Flow Chart of Fidelity Needs Assessment (Part 1) …..................................................... 8.17

8.2 Flow Chart of Fidelity Needs Assessment (Part 2) ............................................................. 8.18

\section{Tables}

2.1 Simulator Fidelity Terminology: A Sampling of Publications ............................................... 2.2

2.2 Knowledge Types and Cognitive Processes ....................................................................... 2.5

3.1 DIF Criteria for Rating the Task/Inventory Items …............................................................ 3.8

3.2 Framework of Types and Levels of KSAs........................................................................ 3.9

3.3 Comparison of the Project and NUREG-0700, Rev. 2 System Taxonomies.............................. 3.10

3.4 CR HSI System Characterization Strategies ..................................................................... 3.14

5.1 Task/KSA Inventory Items Receiving the Highest and Lowest DIF Scores ............................ 5.5

5.2 Highest and Lowest Scores for Difficulty and Importance................................................... 5.6

5.3 Items Receiving the Highest and Lowest Scores for Frequency .............................................. 5.7

5.4 Task/KSA Inventory Items Most Difficult to Learn and for Which Simulator Use is Most

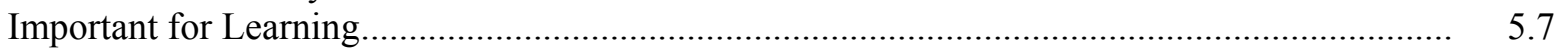

5.5 Ratings of Task/KSAs on Levels of Knowledges and Skills Needed......................................... 5.9

6.1 Example Scope of TTC PWR Simulator Training Course (\#624) ............................................. 6.3

6.2 Range of Years of Experience in Current and Previous Positions ........................................... 6.8

8.1 Ratings on Factors Influencing Fidelity Needs for Training Task/KSA Inventory Items ........... $\quad 8.3$

8.2 Top-Rated Task/KSA Inventory Items in Terms of Training Priority and Other Factors ........... $\quad 8.6$

8.3 Text of Task/KSA Inventory Items Included in Table 8.2 …................................................ 8.7

8.4 Importance of HSI Systems to Task/KSA Items from Table 8.2 ….......................................... 


\subsection{Introduction}

\subsection{Statement of Purpose}

The objective of this study is to determine how simulator physical fidelity influences the effectiveness of training the regulatory personnel responsible for examination and oversight of operating personnel and inspection of technical systems at nuclear power reactors. It seeks to contribute to the U.S. Nuclear Regulatory Commission's (NRC's) understanding of the physical fidelity requirements of training simulators. This study will provide an analytic framework, data, and analyses that inform NRC decisions about the physical fidelity requirements of the simulators it will need to train its staff for assignment at advanced reactors. These staff are expected to come from increasingly diverse educational and experiential backgrounds.

The project activities included a literature review, inventory and characterization of the tasks resident inspectors (RI) and operator licensing examiners (OLE) perform, analysis of the knowledge and skills these staff need to perform their tasks competently, and a training needs assessment, which includes information gathered from a survey of NRC RIs and OLEs and interviews with NRC staff and personnel at other facilities conducting simulator training of examiners and regulators. A series of expert panels reviewed the characteristics of advanced reactor control rooms (CRs) and simulators, rated the importance of the various human-system interface (HSI) systems to the tasks, and assessed the importance of a simulator in RI and OLE training. Figure 1.1 shows the overall structure of the study.

The NRC has an interest in determining the level to which simulator physical fidelity is necessary to support effective regulator training. The effect of training simulators' physical fidelity on regulator performance is currently unknown. Moreover, a simulation's level of fidelity can have a large effect on its cost because the more a simulation approximates the reality of the simulated system, the more it costs to develop, configure, install, operate, and maintain. Because it is expected that advanced reactor CRs will predominately contain digital displays and controls, it is likely that many aspects of the human interaction with the plants can be effectively simulated on computers commonly available outside the CR. With expected new opportunities for simulation-based training of inspectors comes a need to understand the trade-off between simulator training effectiveness and cost.

Research on training has shown that simulation fidelity can sometimes have a positive effect on transfer to the operational environment in various workplaces. However, it has also shown that in some instances there are no significant differences in training effectiveness between high- and low-fidelity simulators, and that high physical fidelity may sometimes impede learning. The conditions in which there are no differences between high- and low-fidelity simulation are often not clear. Moreover, it is likely that some aspects of fidelity are more important than others. In some industries, a continuum of simulation fidelity is used, with low-fidelity part-task simulations used for initial training sessions and fully immersive simulations used for later sessions.

The study seeks to provide information useful to the NRC in developing training programs for staff whose assignments will include advanced nuclear power plants (NPPs). This report deals only with the question of simulator physical fidelity in the training needed to prepare NRC staff for their responsibilities in advanced reactor CRs. The study analysis assumes that the structure and approach of the NRC training will remain as it is now and that role of the NRC Technical Training Center (TTC) training 
simulator in future training will also remain unchanged. The study does not deal with the overall training design for RIs and OLEs, although some of the information generated by the study may be useful to the NRC from a curriculum development perspective. As noted by SECY-08-0096, final development of a training program depends upon completed plant designs, including procedures that have not been finalized and are therefore not yet available.

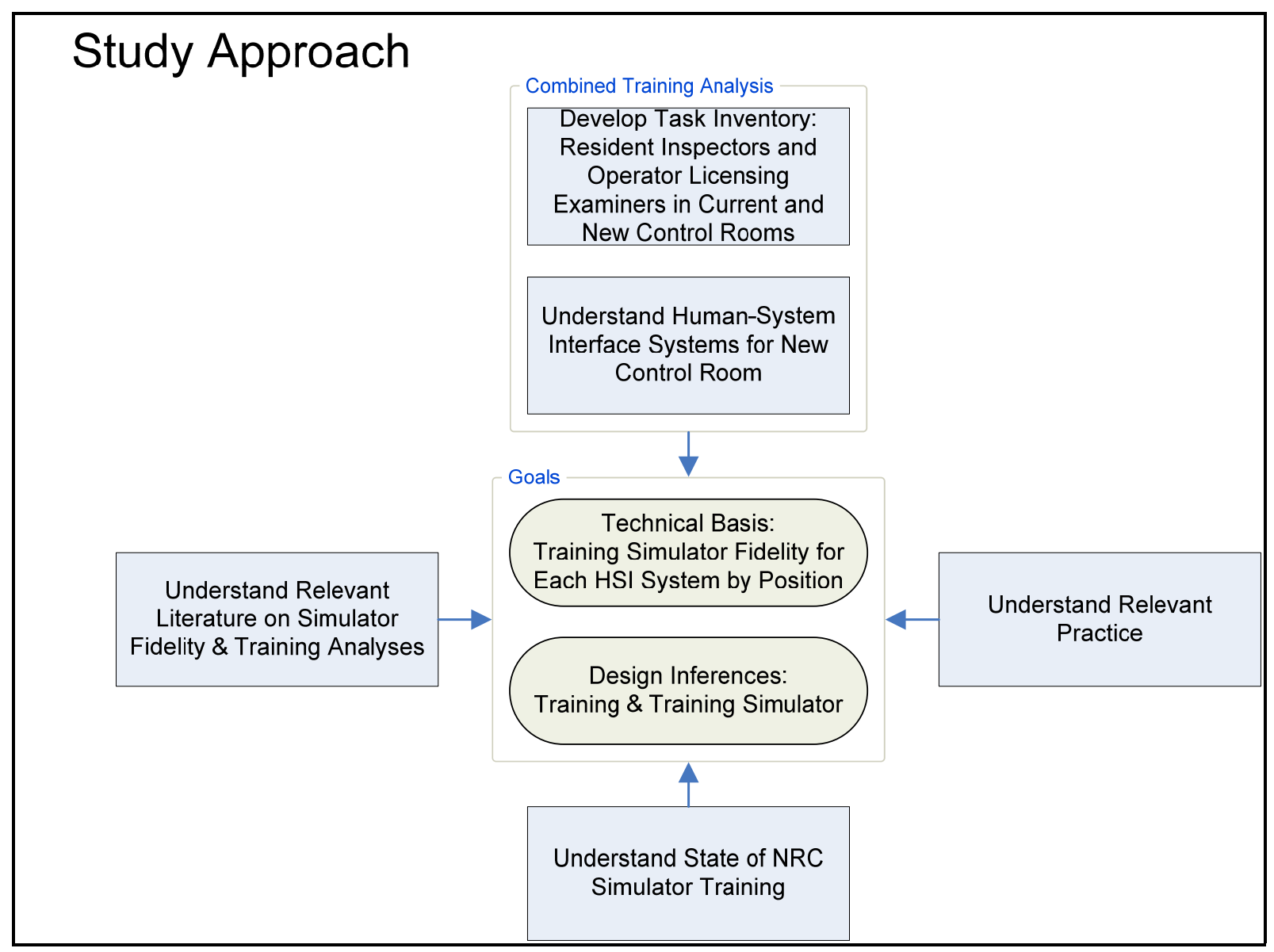

Figure 1.1. Overview of Study Approach

\subsection{Evolution of the Project Focus and Approach}

This project was originally formulated as an academic evaluation of the simulator fidelity requirements necessary for teaching regulatory evaluators to understand the activities and tasks performed by CR personnel. The intent was then to apply the results of this evaluation to the training needs of the TTC for use in determining the type and scope of simulators needed for training regulators for work with the coming generation of advanced reactor types.

Consequently, the initial project activities focused on review of the literature, study of expectations about the characteristics of the new CR human-system interface (HSI) systems, and development of dimensions of physical fidelity pertinent to this problem. In addition, as part of its due diligence, the project team also sought information about other instances in which regulator training utilized simulators. 
Literature on the systematic approach to training (SAT) ${ }^{1}$ persuaded the team that this problem was best addressed by focusing first on delineation of the tasks and knowledges, skills, and abilities (KSAs) to be taught through the training, and only then addressing the technologies needed to support student learning. In addition, the team's review of the literature and discussions with trainers and managers of training simulation facilities worldwide confirmed that the current state of information on simulators in training regulators was inadequate to support the originally envisioned approach.

Consequently, the PNNL team, in consultation with the NRC project manager, refocused its efforts on delineating the learning needs of NRC regulators, particularly RIs and OLEs. This involved designing a process to inventory the tasks and KSAs that RIs and OLEs needed in order to fulfill their job duties, characterize the role that simulator training played in the acquisition of these capabilities, and obtain information from current RIs and OLEs about the impact of physical fidelity differences on job performance and learning. The specific methods used to implement this approach are described in Chapter 3.

\subsection{Background}

Simulator fidelity, the degree of similarity between a simulator and a reference unit; and scope, the amount of systems, subsystems, and components of the reference unit that are simulated; have been the key concerns in the training and testing of NPP operators. Simulator fidelity can be separated into two concepts: "Physical fidelity," the degree to which a simulator's physical characteristics (e.g., look, feel, sound, layout, location, operator interface, etc.) match those of a reference unit; and "functional fidelity," the degree to which the simulator's informational characteristics, including plant parameters during both steady and dynamic states, match those of the reference unit. American National Standards Institute/ American Nuclear Society (ANSI/ANS)-3.5-1998, endorsed by NRC Regulatory Guide 1.149 (which specifies compliance methods for 10 CFR Part 55.46 on the use of simulation facilities) generally requires that operators be trained and examined using plant-referenced simulators of full scope and high fidelity. NRC regulatory staff must understand nuclear facility operations and operators' roles in the CR. Although there is some overlap, control-room related regulatory functions are significantly different from operator tasks. Control-room regulatory functions include, for example, inspection, monitoring, assessment, and reporting on operator and CR crew performance; evaluating the integrity and operability of plant systems and equipment; gathering information and communicating with the NRC during offnormal events. There are a large number of operating reactors in the United States, as well as differences between their CRs, even within the same reactor design. The NRC has previously determined that plantreferenced simulators for training NRC regulatory staff are neither necessary nor feasible.

The NRC's TTC provides reactor technology training that covers the existing span of commercial power reactors currently deployed in the United States. The reactor training provided by the TTC currently focuses on the four vendor designs found in the United States: the Westinghouse family of pressurized water reactors (PWRs), the Combustion Engineering family of PWRs, the Babcock and Wilcox family of PWRs, and the General Electric family of boiling water reactors (BWRs). The CRs for each of these designs rely principally on analog instrumentation and controls (I\&C). The TTC employs four full-scope simulators, one for each of the four families of power reactors in the United States. The TTC simulators are generally divergent in physical fidelity from the CRs and simulators at operating

\footnotetext{
${ }^{1}$ See Chapter 2 for a discussion of the literature on systematic approach to training.
} 
plants, even within the same reactor family. The new reactors for which the TTC must train regulatory staff, such as the Westinghouse-AP-1000, the Toshiba ABWR, the General Electric ESBWR, the Areva EPR, and the Mitsubishi US-APWR, are expected to be substantially more reliant on digital I\&C when compared with currently operating designs, whose CRs are predominated by analog I\&C. They are also expected to be highly standardized. RIs and OLEs, the focal TTC simulator trainee population, are required to attend one two week simulator training course every three years for each family of reactors to which the RI or OLE is assigned.

The HSIs of new CRs will be significantly different from those of existing CRs in the United States. Although highly standardized within each design, the HSIs of the advanced reactors are expected to differ considerably across designs. The use of digital I\&C enables a level of flexibility and integration not possible in existing CRs. Because the new designs use visual display units (VDUs) in place of fixed, spatially separated controls and instrumentation, the design of panels and workstations, the layout of the CR workplace, the overall look of the CR, and how the operating crews interact with the HSIs and each other within the CR area will be much different than in existing CRs. Most controls in advanced reactor CRs will be soft controls on VDUs. Use of hard controllers and switches will be limited exclusively for use as backup in the event there is a common mode failure of the computer or digital network that would render the soft controls inoperative. While there will be fixed-position displays of key parameters in the new designs (some on hard panels, others in a large, group-view display system), operator interaction with HSIs in new CRs will be dominated by the use of single-operator displays, each with numerous pages of information and controls.

A much higher degree of integration of information and controls will be characteristic of new designs: many combinations of information and controls will be accessed both in large, control-room-wide groupview displays and within single pages (and, in some cases, the same visual image) of a many-page, singleoperator VDU. With some designs, I\&C VDUs, alarm VDUs, procedure VDUs, and, potentially other operator support systems will be linked. VDUs will be direct-manipulation graphical user interfaces (GUIs), with human input via mouse and, in some cases, touch-screen. For all new designs, there are expected to be dedicated safety-related VDUs and dedicated hard switches for safety-related controls. The new HSIs will likely include a higher level of computerized operator support including various automatic alarm processing schemes and computer based procedure systems. These changes in CRs and simulators raise the questions of physical fidelity impacts of training simulators that are addressed in this study.

\subsection{Structure of the Report}

Following this introductory chapter, Chapter 2 summarizes the key findings from the literature review and examination of practices at other organizations that train regulators/inspectors/examiners (as opposed to those being regulated/inspected/examined, such as operators or pilots). Chapter 3 describes the methodology of the study. Chapter 4 describes and compares the CRs and human-system interfaces, respectively of the Gen III and Gen III+ CRs for which information was available to the study team. Chapter 5 describes the results of the analysis of the NRC RI and OLE positions and the development of the Task/KSA (knowledges, skills, and abilities) Inventory. Chapter 6 provides a brief summary of the NRC training parameters, with a special focus on simulator training. Chapter 7 presents the results of the on-line survey of current RIs and OLEs and their judgments about physical fidelity differences between the simulator on which they trained and the CR or simulator at the plants to which they are assigned, as well as the impacts of those differences on learning and job performance. Chapter 8 presents findings and 
conclusions regarding training priorities and needs and the implications of the data collected for TTC training simulator physical fidelity. This is followed by a list of references cited in the study. A series of appendices present data-collection instruments, methodological details, data tables, and supporting analyses. 


\subsection{Summary of Key Literature and Practice}

\subsection{Literature and Practice Included in the Review}

This review covers a wide variety of source types including journal papers; conference proceedings; technical reports on research and practice from government, industry, and academic sources; books; book chapters in edited volumes; and interviews with those involved in decision-making with respect to the use of simulators in training oversight tasks. Work domains included not only NPP operations and oversight, but also flight safety inspection, flight training, and a limited number of other domains in which simulator training plays a key role. A large majority of the literature and practice on simulator fidelity is focused on flight training. Sources come from broad topical areas and include a large scope of publications on human factors, psychology, training, educational theory, computer science, and engineering. Simulator training involves a combination of technical and behavioral concerns, as well as their interaction, and necessarily spans a broad topical area.

Sources were initially identified through searches in on-line bibliographic databases, such as Engineering Index, Academic Search Complete, and Web of Science, web-based searches using the various search engines, and reference tracking.

Figure 2.1 shows the steps involved in gaining an understanding of the relevant literature and practice.

\subsection{Simulator Physical Fidelity Concepts and Definitions}

Fidelity in simulation has been addressed in a number of different ways over the years. It has sometimes been categorized into types, with different names applied to the same types of fidelity; with similar, but not identical, descriptions of fidelity types; and with a focus on a particular type or types of fidelity. It has also sometimes been treated as a generic concept without differentiation into different subcategories. A sampling of the various terms and categorizations applied to simulator fidelity is shown in Table 2.1. This small sample of the literature illustrates the variety of these terms and categories and the general lack of agreement on both specifics and concepts among those studying and utilizing simulators. Review of this literature also shows a lack of clear trends or convergence over time. Robert T. Hays's meta-review (1980) of the terms and their usage attempts a definition of simulator fidelity focused on training needs. The concepts of fidelity presented by Hays \& Singer (1989) are among the most frequently cited. Hays and Singer (1989) break fidelity into two-dimensions, both of which concern the degree of similarity between the simulator and that which is being simulated, otherwise called the "simuland" or "reference unit." One of these dimensions is "physical fidelity," which is based on similarity between corresponding physical characteristics of the simulator and the reference unit. Physical characteristics include shape, size, location, look, sound, feel, smell, and taste. The second dimension is "functional fidelity," which is based on similarity between the way a simulation reacts to tasks executed by an operator (or crew of operators) and the way the reference unit reacts to such tasks. This report, which is focused on the use of simulators as training devices, adopts the Hays and Singer (1989) two-dimensional definition. 


\section{Understand Relevant Literature and Practice On Training Fidelity \& Training Analyses}

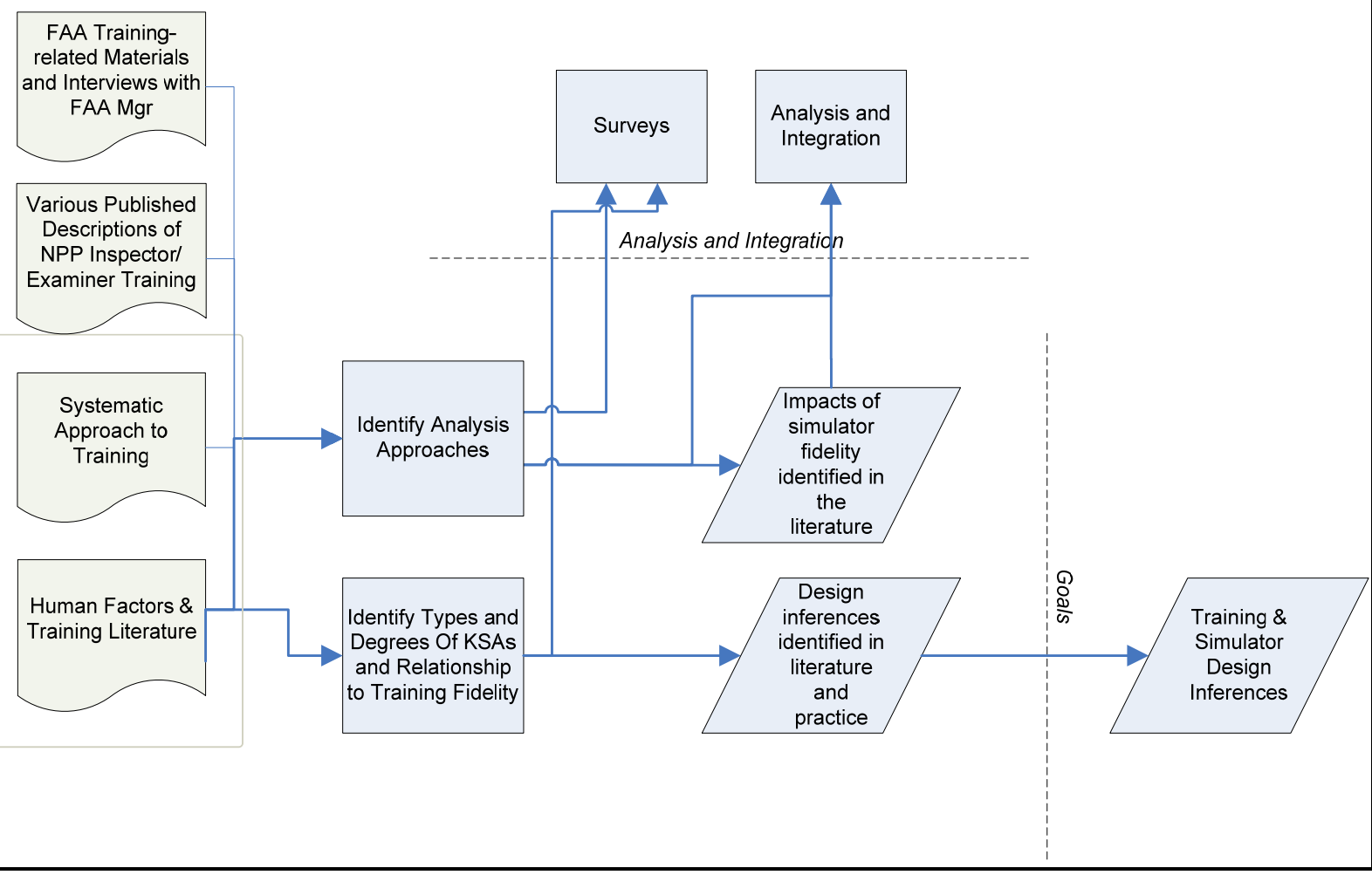

Figure 2.1. Understanding the Relevant Literature and Practice

Table 2.1. Simulator Fidelity Terminology: A Sampling of Publications

\begin{tabular}{|l|l|l|l|l|l|l|}
\hline \multicolumn{1}{|c|}{ Term } & $\begin{array}{c}\text { "Fidelity" } \\
\text { (Generic) }\end{array}$ & "Physical" & "Equipment" & "Functional" & "Environmental" & "Psychological" \\
\hline $\begin{array}{l}\text { Gerathewohl } \\
(1969)\end{array}$ & $\begin{array}{l}\text { "Degree to } \\
\text { which a device } \\
\text { accurately } \\
\text { reproduces a } \\
\text { specific effect" }\end{array}$ & & & & & \\
\hline $\begin{array}{l}\text { Kinkade and } \\
\text { Wheaton } \\
(1972)\end{array}$ & & $\begin{array}{l}\text { Degree to which } \\
\text { the simulator } \\
\text { duplicates the } \\
\text { appearance and } \\
\text { "feel" of the } \\
\text { operational } \\
\text { equipment }\end{array}$ & & $\begin{array}{l}\text { Degree to which } \\
\text { the simulator } \\
\text { duplicates the } \\
\text { sensory } \\
\text { stimulation } \\
\text { (excluding control } \\
\text { feel) which is } \\
\text { received from the } \\
\text { task situation }\end{array}$ & $\begin{array}{l}\text { Degree to which } \\
\text { the simulator is } \\
\text { perceived by the } \\
\text { trainee as being a } \\
\text { duplicate of the } \\
\text { operational } \\
\text { equipment and } \\
\text { the task situation }\end{array}$ \\
\hline $\begin{array}{l}\text { Slenker and } \\
\text { Cream (1977) }\end{array}$ & & & $\begin{array}{l}\text { Attempt to } \\
\text { duplicate all of } \\
\text { the stimulus } \\
\text { conditions of } \\
\text { the actual } \\
\text { equipment }\end{array}$ & & \\
\hline
\end{tabular}


Table 2.1. (contd)

\begin{tabular}{|c|c|c|c|c|c|c|}
\hline Term & $\begin{array}{l}\text { "Fidelity" } \\
\text { (Generic) }\end{array}$ & "Physical" & "Equipment" & "Functional" & "Environmental" & "Psychological" \\
\hline $\begin{array}{l}\text { Fink and } \\
\text { Shriver } \\
(1978)\end{array}$ & & $\begin{array}{l}\text { "Extent to } \\
\text { which training } \\
\text { equipments } \\
\text { duplicate the } \\
\text { appearance and } \\
\text { feel of their real } \\
\text { equipment } \\
\text { counterparts" }\end{array}$ & Same as Physical & $\begin{array}{l}\text { "Extent to } \\
\text { which training } \\
\text { equipments } \\
\text { duplicate } \\
\text { stimuli which } \\
\text { are present in } \\
\text { the operational } \\
\text { environment } \\
\text { and provide an } \\
\text { opportunity for } \\
\text { responding } \\
\text { realistically to } \\
\text { these stimuli" }\end{array}$ & $\begin{array}{l}\text { Same as } \\
\text { Functional }\end{array}$ & $\begin{array}{l}\text { "The extent to } \\
\text { which trainees } \\
\text { perceive the } \\
\text { training } \\
\text { equipment as } \\
\text { being a duplicate } \\
\text { of the operational } \\
\text { equipment and } \\
\text { the task } \\
\text { situation" }\end{array}$ \\
\hline $\begin{array}{l}\text { Freda and } \\
\text { Ozkaptan } \\
(1980)\end{array}$ & $\begin{array}{l}\text { Amount/kind of } \\
\text { similarity } \\
\text { between the } \\
\text { training device } \\
\text { (setting) and the } \\
\text { operational } \\
\text { equipment } \\
\text { (setting) }\end{array}$ & $\begin{array}{l}\text { "Engineering } \\
\text { (hardware) } \\
\text { representation } \\
\text { of features in } \\
\text { the operational } \\
\text { equipment" }\end{array}$ & & & & $\begin{array}{l}\text { "Behavioral } \\
\text { (functional) } \\
\text { representation of } \\
\text { the information } \\
\text { processing } \\
\text { demands of the } \\
\text { operational } \\
\text { equipment" }\end{array}$ \\
\hline Hays (1980) & $\begin{array}{l}\text { "Degree of } \\
\text { similarity } \\
\text { between the } \\
\text { simulator and the } \\
\text { equipment which } \\
\text { is simulated" }\end{array}$ & $\begin{array}{l}\text { "Measurement } \\
\text { of the physical } \\
\text { characteristics } \\
\text { of the } \\
\text { simulator" }\end{array}$ & & $\begin{array}{l}\text { "Measurement } \\
\text { of ... the } \\
\text { informational } \\
\text { or stimulus and } \\
\text { response } \\
\text { options of the } \\
\text { equipment" }\end{array}$ & & \\
\hline $\begin{array}{l}\text { Hays and } \\
\text { Singer (1989) }\end{array}$ & $\begin{array}{l}\text { "Degree of } \\
\text { similarity } \\
\text { between the } \\
\text { training situation } \\
\text { and the } \\
\text { operational } \\
\text { situation which } \\
\text { is simulated" }\end{array}$ & $\begin{array}{l}\text { One of two } \\
\text { dimensions of } \\
\text { the measure of } \\
\text { fidelity, "for } \\
\text { example visual, } \\
\text { spatial, } \\
\text { kinesthetic, } \\
\text { etc." }\end{array}$ & & $\begin{array}{l}\text { One of two } \\
\text { dimensions of } \\
\text { the measure of } \\
\text { fidelity, "for } \\
\text { example the } \\
\text { informational, } \\
\text { stimulus, and } \\
\text { response } \\
\text { options of the } \\
\text { training } \\
\text { situation." }\end{array}$ & & \\
\hline $\begin{array}{l}\text { ANSI/ANS- } \\
3.5-(1998)\end{array}$ & & $\begin{array}{l}\text { "Degree of } \\
\text { similarity } \\
\text { between the } \\
\text { simulator and } \\
\text { the reference } \\
\text { unity, such as } \\
\text { physical } \\
\text { location of } \\
\text { panels, } \\
\text { equipment, } \\
\text { instruments, } \\
\text { controls, labels, } \\
\text { and related form } \\
\text { and function" }\end{array}$ & & & & \\
\hline
\end{tabular}


Table 2.1. (contd)

\begin{tabular}{|l|l|l|l|l|l|l|}
\hline \multicolumn{1}{|c|}{ Term } & $\begin{array}{l}\text { "Fidelity" } \\
\text { (Generic) }\end{array}$ & "Physical" & "Equipment" & "Functional" & "Environmental" & "Psychological" \\
\hline Gross (1999) & $\begin{array}{l}\text { "Degree to } \\
\text { which ... a } \\
\text { simulation } \\
\text { reproduces the } \\
\text { state and } \\
\text { behavior of a } \\
\text { real world } \\
\text { object, feature, } \\
\text { condition, or } \\
\text { chosen standard } \\
\text { in a measurable } \\
\text { or perceivable } \\
\text { manner" }\end{array}$ & & & & & \\
\hline $\begin{array}{l}\text { Feinstein and } \\
\text { Cannon } \\
\text { (2001) }\end{array}$ & $\begin{array}{l}\text { "Level of } \\
\text { realism that a } \\
\text { simulation } \\
\text { presents to the } \\
\text { learner" }\end{array}$ & & & & & \\
\hline
\end{tabular}

\subsection{Knowledge, Skills, and Abilities Frameworks}

\subsubsection{Taxonomies of the Cognitive Domain}

Oversight and regulatory tasks require different types and degrees of KSAs. For example, the type of knowledge needed to understand the process by which a control rod drive system is started up (a procedure) may be different from the type of knowledge needed to understand startup accidents (facts and concepts). Also, the degree to which an inspector needs to remember, comprehend, apply, evaluate, or create critical operator procedures may be different from the degree to which non-critical procedures are understood.

Anderson and Krathwohl's (2001) revision of Bloom's taxonomy has become the cornerstone of curriculum development. This taxonomy is used to guide curriculum development and evaluation, i.e., to help structure or evaluate what is being taught. As shown in Table 2.2, the taxonomy identifies four types of knowledge and six dimensions of cognitive processes that act upon these types of knowledge. While these two dimensions are conceptually independent, they are often crossed (types by cognitive processes) to label specific learning outcomes in designing a training or educational program. For instance, the methods used to effectively train the application (a cognitive process) of a procedure (a knowledge type) can be quite different from the most effective methods for training memory (a cognitive process) of the same procedure. Bloom's original taxonomy and Anderson and Krathwohl's revision are commonly used and cited by training designers and researchers. While there are alternate taxonomies of cognitive activity, such as Biggs's SOLO taxonomy (Biggs \& Collis, 1982) and Porter (2002), none have had an impact on training equal to that of Bloom, Anderson, and Krathwohl. 
Table 2.2. Knowledge Types and Cognitive Processes

\begin{tabular}{|l|l|}
\hline \multicolumn{2}{|c|}{ Knowledge Types } \\
\hline Knowledge Type & \multicolumn{1}{c|}{ Definition } \\
\hline Factual & Basic, accepted information elements or details; terminology; definitions. \\
\hline Conceptual & $\begin{array}{l}\text { Interrelationships among elements; structure; classification; categories; } \\
\text { generalizations; theories; models. }\end{array}$ \\
\hline Procedural & $\begin{array}{l}\text { Knowledge of how to do something; methods; techniques; knowledge of criteria for } \\
\text { selecting procedures }\end{array}$ \\
\hline Meta-cognitive & $\begin{array}{l}\text { Knowledge about how to go about cognitive tasks such as problem solving, } \\
\text { understanding, planning; awareness of one's cognitive competency or level of } \\
\text { knowledge }\end{array}$ \\
\hline Cognitive Process & \multicolumn{2}{|c|}{ Cognitive Processes } \\
\hline Remember & Recognize or recall knowledge from memory \\
\hline Understand & $\begin{array}{l}\text { grasping meaning as exemplified by the ability to explain, interpret, summarize, } \\
\text { compare, infer, etc }\end{array}$ \\
\hline Apply & Use knowledge in a given situation \\
\hline Analyze & $\begin{array}{l}\text { distinguish or differentiate knowledge into constituent parts, distinguish the } \\
\text { relationships between parts, organize parts into a previously understood structure }\end{array}$ \\
\hline Evaluate & Make judgments based on criteria \\
\hline Create & $\begin{array}{l}\text { Organizing parts of knowledge into a new or different structure or whole; } \\
\text { predicting }\end{array}$ \\
\hline
\end{tabular}

\subsubsection{Taxonomies of Skills and Abilities}

Several taxonomies in the psycho-motor domain list the various perceptual modalities that are the subject of training. Visual, auditory, haptic, and motor modalities are the most commonly considered modalities. Because RIs and OLEs do not touch controls in CRs, visual and auditory modalities are most pertinent in the present work. Schneider's model of controlled/automatic processing (Shiffrin and Schneider 1977) is the most commonly used model for specifying levels of psycho-motor ability. Controlled processing is characterized by effortful, inefficient, and inaccurate performance. Automatic processing is characterized by low awareness of the psycho-motor process, effortlessness, efficiency, and accuracy, though skills at the level of automaticity are highly adapted to a particular task and break down when the task inputs or outputs change.

\subsection{Fidelity and Training}

\subsubsection{Fidelity and Transfer}

The focal concern of training, both in practice and in research, is transfer. "Transfer," in training, is when knowledge and skills acquired in one context can be used in a different context. It refers to the extent that existing or acquired skills influence the acquisition, relearning, or performance of a knowledge, skill, or ability. Implicit in most uses of the term is that the knowledge and skills in the learning context will be helpful for performance in the context to which the knowledge and skills are 
transferred. "Positive transfer" occurs when previously learned knowledge and skills help performance. Conversely, "negative transfer" occurs when previously learned knowledge and skills impede performance. Ideally, training system designers manipulate the training context so that the training factors such as environment, lessons, lesson sequencing, materials, objectives, and delivery produce positive transfer in the performance environment (see for example, Gerathewohl 1969; Adams 1987; Reder and Klatzky 1994; Carretta and Dunlap 1998; Alexander et al. 2005; Schwartz and Nasir 2008). Transfer can be measured in terms of the reduction (or increase) in the amount of time a trainee needs to learn in the job situation as a result of training. This is a measure often used to assess the transfer achieved from simulator training in aviation (Patrick 2003:416).

A common supposition is that the greater the similarity of the training context and the performance context, the more likely it is that positive transfer will occur. Taken at face value, such a supposition is reasonable - taken to an extreme, if the training environment actually is the performance environment, it can be inferred that there may be no need for transfer, since there is no need for a trainee to take what is learned "to" any other environment. However, this supposition ignores findings from both research and practice that the training conditions in which learning occurs effectively are often different from the performance conditions (Gerathewohl 1969; Su 1984; Hays and Singer 1989; Roscoe 1991; Lever 2004).

The effort to understand transfer led to the notion of "identical elements," defined by Anderson (1987) as cognitive elements that are the rules that underpin procedural knowledge (i.e., knowing how to do something) and research to understand the attributes that determine whether an element is perceived as identical or not. Subsequent research has shown that it is important for the trainee to perceive the similarity between elements, and therefore that it is important for training to point out the nature of the similarity. Reder and Klatzky (1994) refer to research implying that for training many types of tasks it is sufficient that physical fidelity be only high enough that the trainee can recognize that the "cue" provided in the training simulator is the same "cue" in the performance environment. If the visual context surrounding the cue is similar enough, this can help in matching cues between the training environment and the performance environment, but this recognition is not dependent on high degrees of physical similarity. To prevent negative transfer, this implies that it is also advantageous to point out differences, while clarifying the types of similarities that exist and their level of abstraction (Glick and Holyoak 1987). The recommendation is that during training, the instructor makes the students as aware as possible of the range of tasks and situations in which the new knowledge/skills are relevant. As skills are learned, and repeated, they become more specific and tuned to particular contexts, more automatic, and less demanding of attention. This tends to reduce positive transfer. However, transfer may also be adversely affected if the knowledge/shills are too fragile to withstand the distractions and stress associated with application in the job setting.

Experiments conducted by Goldstone and Son (2005) illustrate the complexity of the transfer process. They describe an experiment involving transfer between two dissimilar computer simulations that are related by the scientific principle of "competitive specialization." Competitive specialization is a concept in complex adaptive systems theory. The simulation in one learning context depicted ants selecting food in a visually realistic way, while the other depicted the ants and food using dots and blobs. The transfer simulation depicted an unsupervised neural network learning to categorize patterns of letters, another illustration of the competitive specialization principle. The participants could manipulate the ant/food simulation, most critically by changing the rate at which each ant moves toward the food and observe how it illustrated competitive specialization. The participants could also manipulate the categorization simulation by modifying how quickly each category becomes similar to the input patterns. Those who 
trained on both versions of the ant/food simulation, with the visually realistic version first, manipulated the rate of category adaption in the neural net simulation to reach the "solution" significantly faster than those who trained only on the visually realistic ant/food simulation, those who trained only on the abstract version, and those who trained on the abstract version first and the visually realistic version second. Those who trained on the two versions of the ant/food simulation, but on the abstract version first, did second best. Interestingly, those who trained only on the visually realistic version of the ant/food simulation performed best when "retested" on the same simulation. For this cognitive learning task, performance within the same simulation was best with the visually realistic version, while in the "transfer" situation, the perceptually realistic version followed by the more abstract version was best. These findings suggest that for abstract conceptual learning, exposing a trainee to multiple types of physical representations can be advantageous for transfer and that more visually realistic representations followed by more abstract representations, i.e., exposing a trainee to more concrete representations followed by more abstract representations can also improve transfer.

In experiments using the same simulations, Goldstone \& Sakamoto (2002) found that those who poorly understood the first simulation had greater positive transfer when the second simulation was not visually similar to the first. They also found that those who poorly understood the first simulation had greater positive transfer when the first simulation used abstract visual representations and the second used concrete visual representation. Their interpretation of these outcomes is that those who poorly understand the initial simulation are more likely to be misled by the superficial visual similarities and aspects of the simulations. This finding is consistent with the transfer findings related to expert/novice differences found by Gick \& Holyoak (1983).

In an experiment on transfer with undergraduate students learning math concepts, Kaminski et al. (2006) found that students learning the concept from examples with abstract representations were able to transfer their knowledge but that those learning the concept from examples with concrete representations relevant to the mathematical concept were hindered in their transfer. Interestingly, students whose learning examples used concrete representations that were not relevant to the concept were able to transfer their knowledge. This suggests that physical fidelity, when associated with the relevant aspects of the concept, may hinder transfer, while physical fidelity that is not associated with the concept may not interfere. Gick and Holyoak (1983) found that, for a number of different types of problems, diagrammatic representation of the problems did not affect transfer. Showing learners two types of examples illustrating the same general principle did have a positive effect on transfer, however. Gick and Holyoak theorize that learners are able to infer a general, abstract "schema" that can be successfully used to solve other problems involving the same principle. Moreover, when combined with diagrammatic representations, multiple examples that are analogs of the same general principle are associated with even higher levels of transfer. However, a verbal statement of the abstract underlying principle of a problem did not transfer well. The implication of this research is that multiple, concrete analogs of the problem provide the basis for learning abstract schemas that can be successfully transferred to other problems.

In experiments using a water purification plant simulator, Gonzalez and Quesada (2003) found that those who learned the relationships between visible aspects of the simulation were able to transfer knowledge to performance tasks better than those who had not learned these relationships. While the relationships relied on visually similar components of the simulator, the relationships themselves were abstract "conceptual" relationships, significant for successful transfer of knowledge. In other research using a thermal-hydraulic process simulator, Quesada et al. (2003) theorize that such learning of relationships occurs implicitly through exposure to these relationships over time. 
The assumption has been made throughout the history of simulator use for training that higher fidelity between the training and performance environments will naturally lead to a higher probability of positive transfer and more effective performance, as demonstrated in the literature describing the early use of flight simulators. Questions about the necessity of high fidelity training simulators began to appear in published literature in 1949 (Jones et al. 1985) and continue to the present (Stewart et al. 2008). Other assumptions are that training simulators are necessary only when training in the actual performance environment is not safe and that the fidelity of training simulators should be limited only by technology and cost. Those responsible for the design of NPP CR simulators to train operators today are not immune to these assumptions. The U.S. nuclear power industry has adopted ANSI/ANS-3.5-1998, with endorsement by the NRC, which requires high levels of fidelity between training simulators and the CRs referenced by them. ${ }^{1}$

\subsubsection{Simulator Use in Training}

Simulators are used in training for a number of reasons. For example, simulators often have a practical reason for use: training in actual performance contexts such as aircraft, surgery, NPPs, satelliteground control, and so on, is prohibitive because untrained performance in such environments creates too much risk with regard to safety and cost. In such cases, simulators can provide a reasonable facsimile that is safe and, with sufficient training, allow transition to the actual performance environment. Another reason simulators are used is that simulators can be controlled for instruction. For instance, lessons can be standardized -simulators can be stopped, slowed down, sped up, etc., and specific scenarios can be run on demand - all allowing support of a specific training program. Also, simulators can be designed to explicitly support training by relaxing the need for the full fidelity and scope of the performance environment. For instance, simulator design can be focused to train a particular task or set of tasks, as with partial-scope simulators, or simulators can gradually build in complexity, allowing the complexity of the learning environment to be adaptive to the level of the trainees.

High fidelity is often associated with higher levels of complexity. Research on training and human performance suggests that more complexity in a training simulator often result in higher cognitive load, which can interfere with learning, especially for novice-level learners. In general, the research suggests that tasks that require a great deal of perceptual process have cognitive demands that may make training too complex for novice-level learners. In this case, training research and practice suggests that, when a task is too complex to be trained well and it can be effective decomposed in to sub-tasks, it is often effective to train sub-tasks using a part-task simulator. Other research findings regarding simulator use in training are shown in the following examples:

- Research on training and human performance suggests that simulator training is not an effective means for memorizing facts. Memorization is best accomplished through rote learning or mnemonic techniques, such as repetition, interactive imagery, and spatial imagery (Kyllonen and Alluisi 1987).

- Research on training and human performance suggests that simulator training is not an effective means of learning individual concepts. However, research on training and human performance and the opinions of RIs, OLEs, and TTC instructors suggests that simulator training is an effective means of learning how to integrate different conceptual knowledge to support real-world tasks. Much contemporary educational theory such as constructivism, experiential learning, problem-based

\footnotetext{
${ }^{1}$ ANSI/ANS-3.5-1998 does not dismiss the supplemental use of low fidelity simulators for training, however.
} 
learning, and project-based learning are based on the observation that such high-level learning often occurs in realistic learning environments such as simulations (De Jong and Van Joolingen 1998; Gerjets and Hesse 1994; Gredler 2004)

- Decades of research and practice on training and human performance, particularly in the aviation domain, suggests that simulator training is effective for learning procedural knowledge and psychomotor skills. Moreover, practice of procedural and psychomotor skills in a perceptually and functionally rich context (such as that provided by high-fidelity simulators) is necessary for learning a skill to automaticity. However, a high number of practice repetitions is also necessary for learning a skill to automaticity.

Though there was not strict agreement in the published literature on the levels of simulator physical fidelity needed for training, this study and empirically-based studies in the published literature seem to converge on some relevant findings:

1. Much of the literature on the practice of simulator training suggests that higher fidelity is almost always better than lower levels of fidelity for training, but does not specify why higher fidelity is better or the conditions under which higher fidelity is better. Current NRC regulations generally require full-fidelity, full-scope simulators for training and licensing operators.

2. Research on training and human performance suggests that high levels of fidelity are generally required for training psychomotor skills and procedural skills to fluidity/automaticity. Training psychomotor skills and procedural skills to the point of fluidity/automaticity using a simulator is possible if there is either a single performance setting or a set of performance settings that has a high degree of consistency of and correspondence between perceptual cues and 'correct' behavioral responses.

3. Research on training and human performance suggests that, for training skills that require a reliance on a consistent mapping between perceptual cues and behavioral responses for competent performance, high levels of simulator fidelity to one reference unit can impair transfer of skills learned in simulator training to other systems, even of the same type, if there is different information display and control.

4. Research on training and human performance suggests that novices will often implicitly learn relationships between perceptual cues and system states if relationships appear consistent. If not corrected, this knowledge will often transfer to the performance environment and can result in undesirable actions if there are perceptually similar cues between the training context and the performance context, even when recall and application of relevant conceptual knowledge would more likely result in desirable actions.

\subsubsection{Impacts of Simulator Physical Fidelity on Training Outcomes}

Numerous studies, both in research and practice, have demonstrated that there is not a clear relationship between high levels of physical fidelity and high positive transfer. Some of these studies indicate that high levels of fidelity can result in negative transfer. Rankin et al. (1984a, 1984b), Su (1984), Jones et al. (1985), Alessi (1988), Hays et al. (1992), Stewart et al. (2008), and several other sources document many instances where either no transfer or negative transfer results from high levels of fidelity. Moreover, the literature also documents many instances in which low fidelity simulators result in higher levels of positive transfer than result from high level fidelity simulators when training the same tasks. 
The factors governing how the level of physical fidelity affects transfer are not straightforward or well understood. Empirical evidence that there is a direct relationship between physical fidelity and transfer is lacking: there is currently no reliable source of guidance for training or simulator designers concerning either the optimal or minimal level of fidelity needed to train for specific tasks. Much of the research suggests that physical fidelity interacts with other aspects of training, including the type of task to be trained (e.g., motor control, following procedures, decision-making, or analysis), the performance level the training is targeting, the nature of the perceptual cues or inputs required for the task, the prior level of proficiency of the trainee (e.g., novice, experienced, expert), and the complexity of the performance environment and training program.

There is evidence that training for psycho-motor tasks can benefit from simulators with high levels of fidelity, often mediated by the simulator's scope (part-task simulators with high fidelity are often effective for training psycho-motor skills). Psycho-motor skills that need to be performed quickly and accurately are typically the subject of training. Acquisition of such skills requires training that provides 5high repetition with clear mappings between perceptual inputs and psycho-motor responses (Shiffrin and Schneider 1977; Schneider 1985; Adams 1987). While clear mappings between perceptual input and response imply high levels of physical fidelity, recent research and practice in the training of surgical skills show that novices can benefit from training programs that start out with lower levels of fidelity and increase the level of fidelity as skill is acquired (Scerbo and Dawson 2007).

As discussed in Chapter 5 of the report, RIs and OLEs need the psycho-motor skills they employ in their jobs at a level of familiarity, but there is no evidence that these skills need to be learned to the level of automaticity. Thus there seems to be no need for high fidelity when training these skills to RIs and OLEs. The findings that show a positive relationship between physical fidelity and transfer in training are chiefly in domains that require a high level of psycho-motor skill for proficiency, such as flight training and surgery. When performance proficiency is required for cognitive skill, physical fidelity often seems to have negative or no effect on transfer-functional fidelity, which is necessary to perform the tasks to be learned in the training environment, seems to be much more highly related to positive transfer. Later sub-sections of this review discuss the positive effects of functional fidelity and negative effects of physical fidelity in learning cognitive skills.

\subsection{Representation of Knowledge and Expert vs. Novice Performance}

One focus of cognitive science and educational research is the difference between experts and novices and how people learn to represent their knowledge so that they can solve problems effectively. Many of those the NRC will be training with simulators will have little or no experience in a CR, but may have good knowledge of the underlying systems in NPPs. There will also be many who have a great deal of experience in existing CRs, good knowledge of the underlying systems in NPPs, but little experience with new CRs based on digital instrumentation and controls (I\&C). Significant changes are anticipated in the demographic and experiential characteristics of future staff compared to current NRC staff. Differences between experts and novices have implications for training goals, content, and strategy, which must be understood and matched with the characteristics of the trainee population.

The extensive literature on differences between novices and experts points to the importance of understanding and considering these differences when planning and implementing training programs. 
Also highlighted is the fact that the same training techniques and equipment may be differently effective with learners at different places along the novice-expert continuum. While not all the studies in this review use a simulator for training or are even focused on training, they address the importance, and complexity, of understanding that which students already know and that which they need to know and be able to do in order to perform their tasks proficiently. For example, when solving physics problems involving classical mechanics, Chi et al. (1981) found that experts represented physics problems according to their abstract, underlying physics principles while novices focused on the 'literal, superficial features' of the given problems. In understanding complex systems Hmelo-Sliver and Pfeffer (2004) found that novices focused on perceptually available and static components of the system, while experts integrated structural (system components), functional, and behavioral (mechanisms) aspects of the system.

\subsection{Training Design}

\subsubsection{Systematic Approach to Training}

Systematic approaches to training ${ }^{1}$ (SAT) typically have an initial analysis component which can be a basis for further development of a training program (IAEA 2000; DOE 1995). A systems approach ensures a comprehensive training process that remains focused on the needs of the organization. The SAT process typically includes the following phases (DOE 1994; Piskurich et al. 2000; Buckley and Caple 2004):

1. Analyze the organization's needs and identify training goals which, when reached, will equip learner's with the knowledge and skills to meet the organization's needs. Usually this phase also includes identifying when training should occur and who should attend as learners.

2. Design a training system that learners and trainers can implement to meet the learning goals; typically, this includes identifying learning objectives (which culminate in reaching learning goals), necessary facilities, necessary funding, course content, lessons and sequence of lessons.

3. Develop a training "package" of resources and materials, including, for example, developing audiovisuals, graphics, and manuals.

4. Implement the training package, including delivering the training, support group feedback, clarifying training materials, administering tests, and conducting the final evaluation. This phase can include administrative activities, such as copying, scheduling facilities, taking attendance data, billing learners, and so on.

5. Evaluate training, including before, during and after implementation of training.

The analysis component involves identifying the KSAs and corresponding tasks that are used in the job addressed by a training program and, once identified, selecting the tasks that will be trained. Some items may require formal training, while others may not-moreover, organizations have limited resources for training and must choose which tasks are most valuable to train. While there are different methods for prioritizing the numerous tasks of a given position, perhaps the most common formal method is called "DIF" analysis, described below.

\footnotetext{
${ }^{1}$ Alternatively called "systems approach to training."
} 


\subsubsection{DIF Analysis to Identify Priority Items for Training}

DIF is an acronym that stands for "Difficulty, Importance, and Frequency," and refers to the difficulty of performing or learning a task, the importance of the task, ${ }^{1}$ and the frequency of task performance. Subject matter experts (SMEs) then rate each of the tasks identified for a job on each of these three dimensions. Since tasks that are difficult to perform tend to require training, tasks rated high in difficulty tend to be selected for training. Similarly, since tasks that are relatively high in Importance are critical for job goals, tasks that rate high in Importance tend to be selected for training as well. Although subject to more frequent modification in rating, the frequency of a task is generally considered to have an inverse relation to priority in training. The rationale is that tasks that are performed infrequently tend to be more critical to train because there are fewer opportunities for learning and retraining infrequent tasks on the job and that they are therefore more likely forgotten. Consequently, tasks that are important but performed infrequently benefit more from training and therefore tend to be given a higher priority for training than those more commonly performed. In DIF analyses, the three ratings are combined to create a single priority measure for each task, typically using a decision tree.

In establishing the priority of tasks/KSAs for training, a scale is developed for each of the three dimensions (difficulty, importance, and frequency). Typically, 2-5 point scales are used (e.g., high-low; high-medium-low), although finer distinctions can be made if a large number of tasks/KSAs are being rated and considerable information about each Task/KSA is available. Numerical values are often used in these scales so that the resulting rating scores can be combined, normalized, and sorted.

Each Task/KSA is then rated on each dimension, according to these scales. Tasks that receive the highest "difficulty" rating, the highest "importance" rating, and the lowest "frequency" rating would receive the highest priority for training. Similarly, tasks that have the lowest "difficulty" rating, the lowest "importance" rating, and the highest "frequency" rating would receive the lowest priority for training. Traditionally, the particular branching points of a decision tree in curriculum development are decided based on reference DIF analyses in an organization's materials on training approaches.

Alternatively, training analysts may adjust the weights given to the three dimensions depending on their relative value in rating the job's contribution toward organizational goals. An example of a DIF analysis framework is shown in Figure 2.2.

Once the rating scores have been assigned to the tasks/KSAs, they can be sorted using Euclidean length of the vector (D, I, Inverse-F), where D, I, and F can range from 1 to 5 (integers). The results can be scaled such that the minimum length vector is scaled to 0.5 and the maximum length vector is scaled to 10.49. The scaled values can be rounded to integers so a DIF value between " 1 " (lowest training priority) and " 10 " (highest training priority) can be assigned to each Task/KSA. While a strictly mathematical approach to an integrated DIF score is atypical, this scale has the advantage of having desirable psychometric properties. $^{2}$ Alternatively, this same treatment can be applied to the range of possible rating scores, and that value applied directly to the Task/KSA (i.e., instead of assigning the rating scores to a task, translate the rating scores into the scaled score table, and assign that value to the task).

\footnotetext{
${ }^{1}$ In the case of NRC regulatory oversight, 'importance' means 'significance for safety'.

${ }^{2}$ As discussed in the DIF analysis section of Chapter 3, this scaling was done with the scale, i.e., using all the possible combinations of DIF ratings $(5 * 5 * 5$ combinations), calculating the Euclidean length, scaling the lengths, and rounding to create a scale that can be represented as a decision tree.
} 


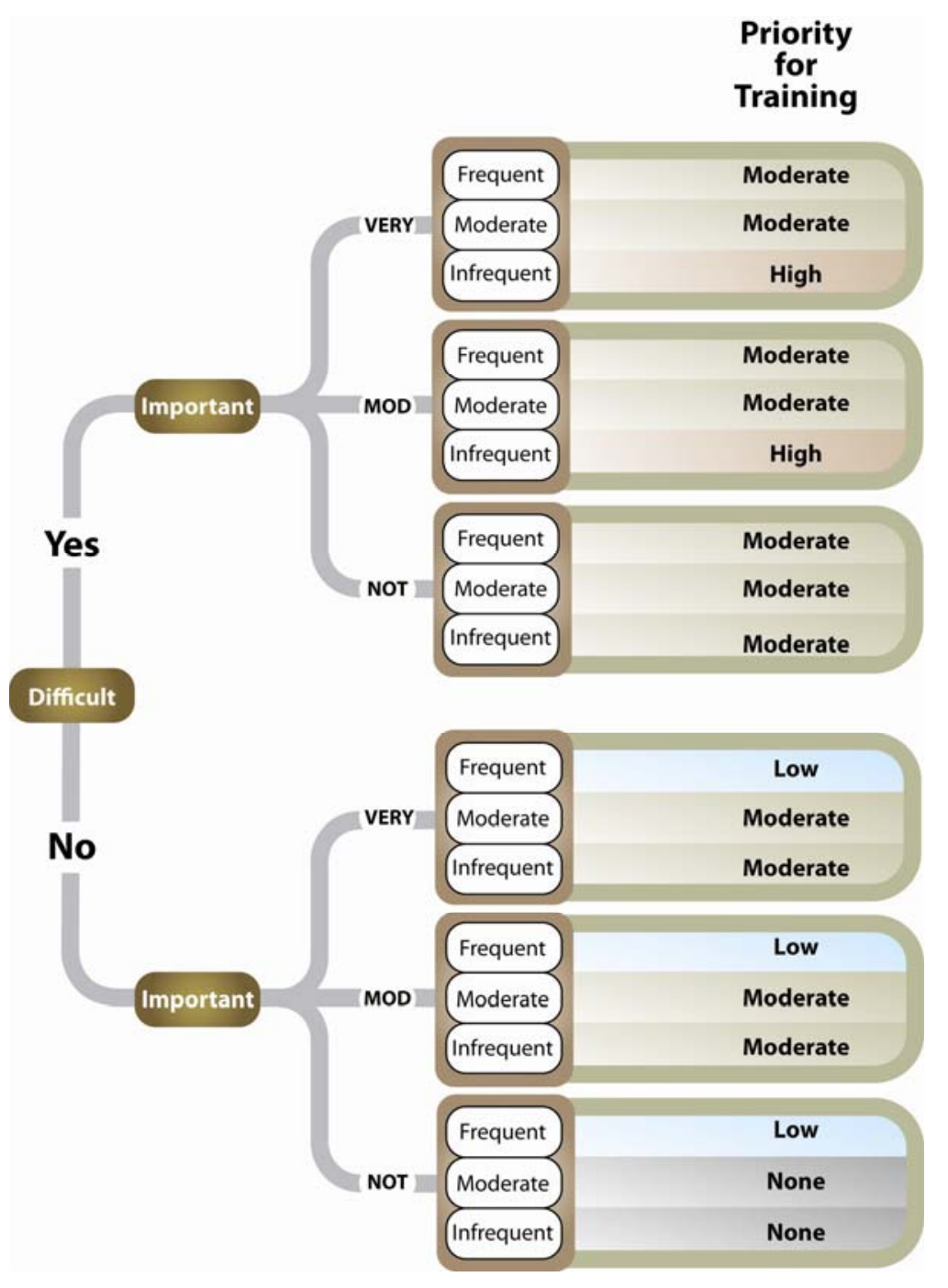

Figure 2.2. Illustration of a DIF Framework

\subsection{Experience Gained from Practice: Simulator Training of Oversight Personnel}

\subsubsection{FAA Flight Safety Inspectors}

Interviews with FAA personnel responsible for simulator training of Aviation Safety Inspectors (ASIs) for the FAA's Flight Standards Service revealed that simulators with varying levels of fidelity are used in their training. Typically, ASIs come to their job with flight experience. The FAA requires that ASIs maintain "flight standards" and that the ASIs be familiar enough with the technology in a particular cockpit that they can competently give practical tests and check rides. Such tests include, but are not limited to, initial certification of general aviation pilots, evaluations for continuing competence, initial flight instructor certificates, and airline transport pilot ratings. Because some of the tests are administered in the air with a single, often inexperienced, pilot, ASIs have to be able to take control of the aircraft and return it safely to an airport to prevent an accident or incident. 
As with forthcoming CRs in NPPs, HSI systems in cockpits have changed from primarily analog displays and controls to "flat-panel glass" and "fly by wire" displays and controls. Currently, both new commercial and general aviation aircraft are typically delivered with flat-panel displays or, for commercial aircraft, configured with flat-panel displays by the airline. Current ASIs who do practical tests of pilots typically have many years of experience and thousands of hours in aircraft with analog avionics. In general aviation there are currently three major suppliers of digital avionics for cockpits, each of which is quite unique. ASIs in commercial inspections typically see a smaller variety of aircraft over an extended time and typically can maintain flight competence by logging sufficient time in the high-fidelity simulators the airlines use to train their own pilots - simulator time contributed by the airlines. Otherwise, the opportunities and resources for training ASIs for proficiency with new cockpit technologies are few. A flight aviation inspector in general aviation often does inspections in many types of aircraft.

The FAA currently contracts with different flight schools around the country to provide training to ASIs in general aviation. Each flight school typically has a focus on one type of digital avionics system, with high- and low-fidelity simulators applicable to the avionics system in which the flight school specializes. High-fidelity simulators are typically limited to a particular aircraft, while in the field, ASIs may see the same avionics systems, but in different aircraft. Also, each flight school can expose the ASIs to the other types of digital avionics systems typically used in general aviation and course materials to point out the differences between the different avionics systems, but ASIs will typically get high-fidelity training on only one digital avionics systems. They will not be able to get training on another system in a high-fidelity simulator until their next scheduled training session, which are typically given at intervals of 2 years.

\subsubsection{Nuclear Power Regulatory Personnel Outside of the United States}

The responsibilities of NPP CR personnel inspection and licensing vary greatly in different countries. In 1998, when the NEA carried out a comparison of inspection and licensing practices for CR operators and shift supervisors in fourteen Organisation for Economic Co-operation and Development (OECD) countries, one of these countries was the United States (NEA/CNRA/R(98)1 1998). In seven non-U.S. countries, inspections and licensing were carried out by a regulatory body or another government body in consultation with a regulatory organization. In one country shift supervisors were licensed by a regulatory body while operators were licensed by the operating organization. In the remaining five countries, the operating organization is responsible for licensing. In Sweden, no specific licenses for CR personnel were needed. Simulator examinations were required for licensing in nine non-U.S. countries; in five of these countries the simulator examinations were carried out by the regulatory or other government organization. With respect to inspections of CR operator training and competence, four non-U.S. countries reported on-site inspections of CR personnel competence; the remaining countries either did not report that on-site inspections were carried out or were unspecific about their practice of inspecting the competence of CR personnel. While operator training on full-scope, replica or relatively high-fidelity simulators was generally either required or used, few had on-site simulators.

In 2001, the International Atomic Energy Agency (IAEA) issued a report describing "good practices" and practical examples for developing training programs for regulatory staff (IAEA-TECDOC-1254 2001). The report did not include any recommendations with respect to simulator training. The report 
did present some example training programs currently being delivered in various countries. For non-U.S. countries, Finland and France reported the use of simulator training for their inspectors.

Finland reported on the period for 1991-1997, during which an inspector training program was designed and implemented. During this period one simulator course was given in each year between 1993 and 1996, each course focusing on a different topic. The courses in 1993-1994 were each 21 hours and those in 1995-1996 were each 14 hours. Two courses used BWR simulators; the other two used VVER (Soviet-developed PWRs) simulators. Between 14 and 16 inspectors participated in each simulator course.

France requires that Inspectors have 2 weeks of full-scope simulator training and 1 week in an "engineering" simulator in order to advance to senior inspector. No simulator training is required for inspectors other than that needed to advance to senior inspector. After becoming senior inspector, France recommends an additional week of training on an "engineering" simulator.

Project staff interviewed management staff at nuclear power training centers in Germany and Spain. Germany conducts all operator and regulator simulator training at a central facility. This facility has sitespecific replica simulators for all NPP CRs in Germany. German regulatory staff receive simulator training at the simulator center, but not necessarily on simulators that reference sites where they work. Spain, which has relatively few power reactors, has recently moved its site-specific simulators from a training center to the reference sites. Regulators currently receive on-site training at these site-specific simulators. 


\subsection{Methodology}

\subsection{Introduction}

This chapter describes the research and analytical methods PNNL used to acquire, analyze, and integrate the information presented in this report. The major methodological foci were on the design and conduct of a training needs assessment for NRC staff being prepared to regulate the next generation of NPPs, the description and comparison of the CR and simulator HSI physical characteristics of current and third generation power reactors, and the impact of differences in physical fidelity on training effectiveness. Together, the analyses are designed to answer the following pertinent question: what attributes must a new reactor plant simulator have to prepare students for their respective duties?

Throughout the project, the PNNL team drew upon the knowledge and first-hand experience of the project team members with TTC training and NPP simulators. The project team consisted of three PNNL staff experienced as OLEs and inspectors, one Human Factors expert, one educational expert, and one expert operator trainer for current U.S. boiling water reactors (BWR) and the upcoming Lungmen Advanced BWR (ABWR) Plant, who is also familiar with new plant CRs and simulators. Other internal PNNL experts on human factors and simulators were consulted as necessary.

The primary sources of information for this project include the following:

- Published human factors and training research relevant for training technology

- Published research and descriptions of practice on the physical fidelity of training simulators

- Documents from the NRC and other agencies with interest in the training needs of nuclear reactor oversight personnel describing the tasks of RIs and OLEs or similar positions

- Documents from the NRC on the knowledge and abilities needed by operators

- Various information on CR HSI technology, both current and new

- Current NRC RIs and OLEs

- Current trainers and other staff at the NRC TTC

- A U.S. Federal Aviation Administration manager responsible for the simulator training program for Aviation Safety Inspectors.

The major elements of the study are illustrated in Figure 3.1. Because some of these elements were undertaken in parallel, the sequence of presentation below does not necessarily represent temporal chronology.

\subsection{Control Room Task and KSA Inventory}

To support the evaluation of the physical fidelity needs for new training simulators, the project team applied a training needs assessment approach. The first step in assessing training needs is to determine that which needs to be trained. After reviewing available information, PNNL determined that it needed to develop this information as part of the project. Discussions with the NRC project manager led to agreement that the tasks performed by RIs and OLEs encompassed the vast majority of regulatory tasks 
performed in the CR and reflected the large majority of NRC personnel who receive CR simulator training. PNNL therefore focused the training needs assessment on these two positions.

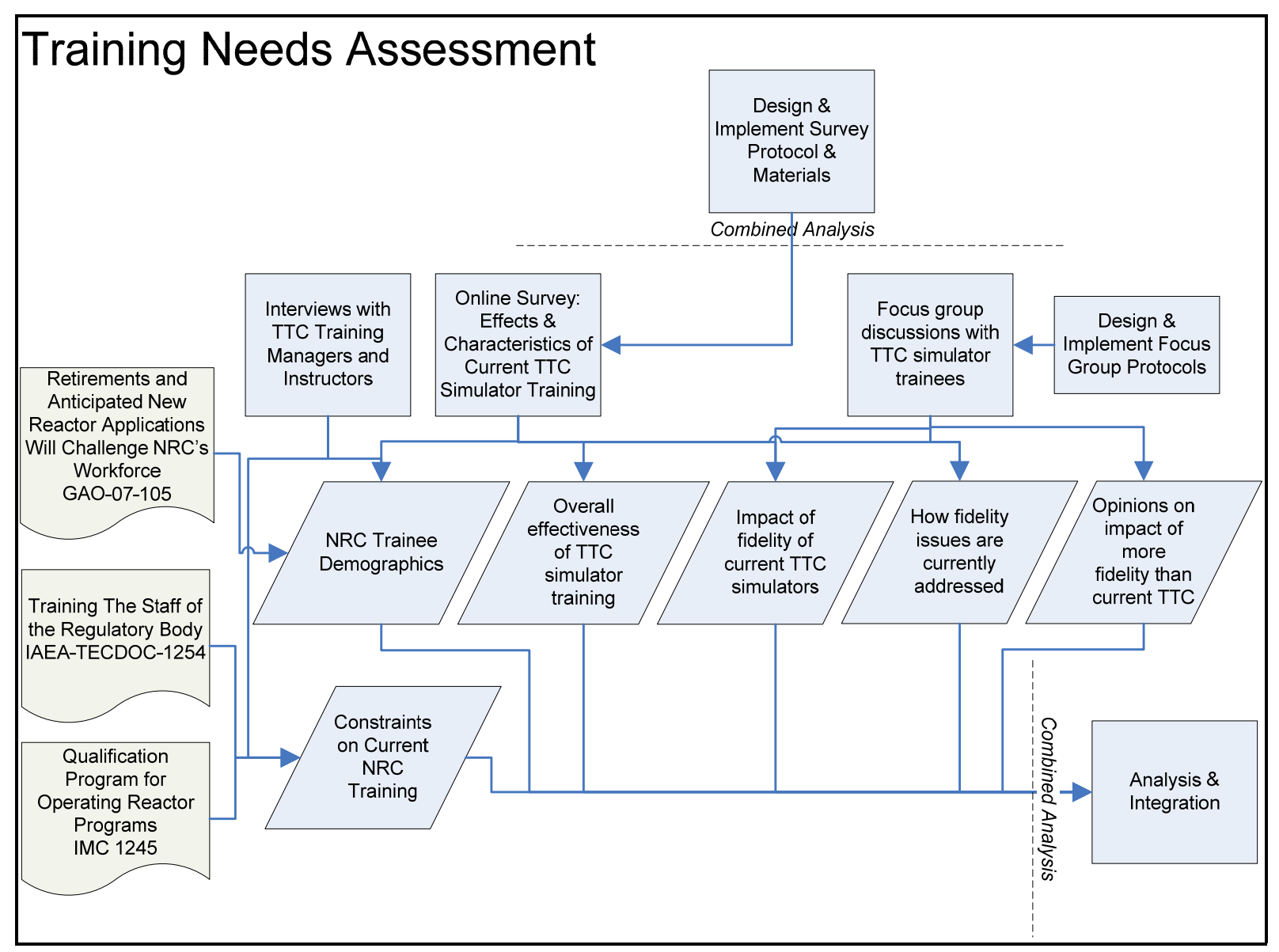

Figure 3.1. Outline of Overall Study Elements

The project scope and budget did not allow a rigorous job-task analysis of the RI and OLE positions. Therefore, the project team devised an alternative approach that drew on the detailed job-task analyses of the reactor operator (RO) and senior reactor operator (SRO) positions and the knowledge and abilities catalogs (KA catalogs) developed for reactor operators of BWR and PWR plants. The initial steps in this analysis are independent of the technology or approaches possible for training these tasks. The later steps address the role of simulators and simulator training in the acquisition of these competencies.

Figure 3.2 shows the steps involved in the task inventory and KSA development process.

\subsubsection{Inventory of Operator Tasks of Regulatory Significance in the Control Room}

To develop the inventory of operator tasks of regulatory significance, the project team drew primarily on NUREG-1122, Knowledge and Abilities Catalog for Nuclear Power Plant Operators: Pressurized Water Reactors and NUREG-1123, Knowledges and Abilities Catalog for Nuclear Power Plant Operators: Boiling Water Reactors (KA Catalogs). These NRC documents are the evolutionary 
outgrowth of a PNNL report, PNNL-5291, Knowledges and Abilities Required of Nuclear Power Plant Operators; Pressurized Water Reactors, prepared in 1984 (Gore et al. 1984a, 1984b). PNNL-5291 represented the results of an extensive job-task analysis of the RO and SRO responsibilities, and research performed by INPO on these positions. Members of the project team previously worked on the projects leading to these publications and, drawing on this experience, along with extensive experience employing these documents to create content-valid operator licensing examinations, developed an inventory, taxonomy, and the KSA inventory of CR-based operator tasks of regulatory significance.

\section{Task Inventory Development}

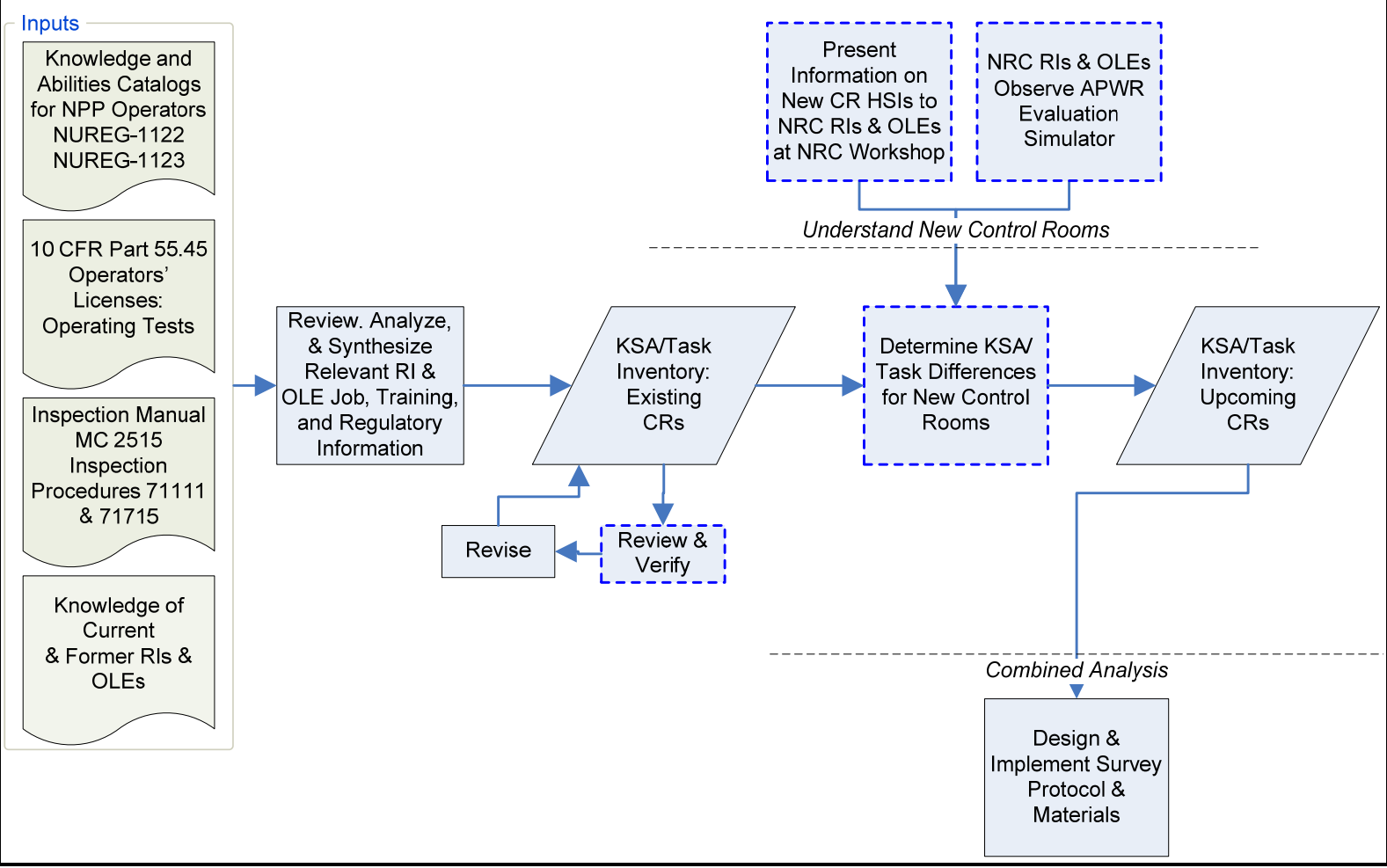

Figure 3.2. Steps in the Task Inventory Development Process

NUREG-1122 and NUREG-1123 list over 5000 specific knowledges, skills, and abilities. The PNNL team determined that this was a much too detailed articulation for the purposes of this project. The level of detail in the lists reflects their development as guidance for licensed operator training. Consequently, the PNNL team determined that it needed to focus on knowledges and abilities, given that the skills required of regulators were different from those required by operators, and that it needed to collect and aggregate the detailed operator KSAs into an exhaustive, manageable inventory of CR responsibilities for operators, and therefore, from a regulatory perspective, are pertinent to NRC RI and OLE trainees. The PNNL team drew upon the "stem statements" used to organize the KSA catalog. The catalog provides "stem statements" for all systems, emergency and abnormal procedures, generic activities not specific to systems or procedures, conduct of operations, and radiological aspects of operation. In addition, the stem statements were formulated to be applicable to multiple systems and multiple reactor designs, an important consideration for this project. The project team used these stem statements and the KSAs listed in NUREG-1122 and NUREG-1123 to develop a preliminary task inventory of operator tasks of 
regulatory significance in the $\mathrm{CR}$. To condense the list into a manageable size, the team grouped the knowledges, skills, and abilities together with respect to intent. The goal was to have a parsimonious list that still captured all the dimensions of the CR activities of regulatory significance.

\subsubsection{Conversion of Operator Tasks/KSAs into Regulatory/Oversight Tasks and KSAS}

The project team then analyzed each item on the condensed list of operator tasks of regulatory significance in the $\mathrm{CR}$ and converted the inventory items into corresponding regulatory/oversight tasks and KSAs applicable to RIs and OLEs. They drew upon the training and experiences of the team members in developing content-valid licensing examinations and justifying performance evaluations of candidates, and also on experiences working as members of NRC teams performing special and enhanced inspections of utility operations.

\subsubsection{Validation of the Task/KSA Inventory for RI and OLE Positions}

The project team compared the converted Task/KSA Inventory to the inspection responsibilities of RIs, as they are described in the NRC Inspection Manual, MC 2515, including Attachments 1, General Overview of the Inspector Training and Qualification Program; 2, Inspector Competencies; and 3, General Orientation Topics. They also compared the inventory items against the more detailed information in Inspection Procedures, including IP 7.1111, Reactor Safety - Initiating Events, Mitigating Systems, Barrier Integrity; IP 71111.11, Licensed Operator Requalification Program; IP 7.1111.22, Surveillance Testing; and IP 71715, Sustained Control Room and Plant Observation. Lastly, they compared the items against 10 CFR 55.45(a), which presents the requirements for operating tests for reactor operators (ROs) and senior reactor operators (SROs) to ensure that each of the test requirements of 10 CFR 55.45(a) was represented in at least one of the tasks in the inventory.

To validate the completeness of the task inventory, a very detailed comparison was conducted with the training objectives of the TTC PWR Course \#624P, which is presented in Appendix D, and with NUREG-0700, Rev. 2, Human-System Interface Review Guidelines. This and other cross-checking, also shown in Appendix D, identified no tasks that were not in the inventory, and no tasks for which a corresponding responsibility was identified.

As an additional validation, the PNNL team then conducted a series of focus group discussions at TTC with a class of OLEs and RIs, including the instructor. In the focus groups, the participants discussed the inventory of tasks/KSAs, evaluated its completeness and the validity of the items, and suggested how it could be improved (see description of the focus group discussions in Section 3.2.4.1 and Appendix A). In the discussion of each Task/KSA Inventory item the participants suggested several wording adjustments and recommended broadening the scope of several Tasks/KSAs. PNNL then asked the participants to review the entire inventory to verify its completeness and ensure that it completely encompassed RI and OLE regulatory responsibilities in the CR. The participants recommended adding a task to address the relatively new risk assessment activities required of the plant. With these changes, the participants agreed that the inventory was valid and complete.

In another analysis that served both to validate the Task/KSA Inventory and provide information about the role of CR HSI systems, the project team assembled an expert panel to review the Task/KSA 
Inventory items in relationship to the elements of current CR HSI systems and elements, and potential new features of advanced reactors. This review helped develop the HSI system taxonomy (discussed in Section 3.5.3) and also served as an additional validation that all the Tasks/KSAs necessary to use the information provided by the various HSI system elements were addressed in the inventory. The results of this analysis are discussed in Chapter 5 and shown in Appendix D.

The team further validated the applicability of the Task/KSA Inventory items to both current and new reactors designs by reviewing the advanced reactor designs (as described in Section 3.7), discussing regulatory responsibilities with NRC personnel and trainers familiar with the reactor and proposed CR designs, and reviewing the inventory items with NRC staff at an NRC workshop, as described in Section 3.2.4.2. By this process, the team concluded that the inventory spanned all of the CR inspection requirements of the RIs and responsibilities of the OLE, and that each task in the inventory is germane to the jobs of the RIs and OLEs. The team concluded that this validation process confirmed that the Task/KSA Inventory provided a valid characterization of what RIs and OLEs need to know for assignments at both current and advanced reactors.

\subsubsection{Taxonomy of the Tasks/KSA Inventory Items}

The PNNL team then analyzed the Task/KSA Inventory items to develop a taxonomy to group them into categories. Examination of the inventory identified four types of items:

- knowledges: understanding the design and operation of the plant, recognizing and addressing malfunctions and upsets, and complying with regulatory requirements ${ }^{1}$

- operation of the plant and its systems

- management of staff resources, personnel, and communications between personnel

- licensing and compliance with regulatory requirements.

Once the taxonomy was developed, the PNNL team categorized the items in the task inventory into these four groups. By this process, the team determined the final inventory of Tasks/KSAs, which is presented in Section 5.1.

\subsubsection{Discussions with NRC Staff and TTC Trainees}

The NRC has a great deal of experience using simulators to train their regulatory and oversight personnel, and NRC staff consequently have experience using simulators to learn these tasks and KSAs. The PNNL team drew upon this experience through a meeting with instructors and two classes of trainees (one BWR and one PWR class), an online survey of current NRC RIs and OLEs, a discussion with NRC staff at an NRC workshop on Gen III and Gen III+ CRs and simulators, and by enlisting NRC experts to participate in expert panel ratings.

\footnotetext{
${ }^{1}$ It was later recognized that five of these knowledges had to do with the condition of the plant and were therefore in a somewhat different category than the remaining knowledge items.
} 


\subsubsection{Focus Group and Informal Discussions with TTC Instructors and Trainees to Validate Inventory, Taxonomy, and Training Approach}

Focus Group Discussions. To obtain first-hand information about the TTC training and its simulators, discuss NRC staff experience and views with simulator training, and pretest a paper copy of the online survey instrument, three members of the PNNL team travelled to the TTC in Chattanooga, Tennessee, for discussions with two classes of NRC staff trainees (RIs and OLEs) and TTC instructors. The focus group meetings were scheduled with the assistance of the NRC project manager. The team also held informal discussions with individuals and small groups of trainees and instructors about the evolution of the training curriculum, observed a class doing simulator training, and made presentations to the instructional staff on the Lungmen reactor and to the groups on HSI technologies that may be used in new reactors. The PNNL team asked the trainees and instructors to discuss the following topic areas at length:

- The instructors were asked to validate each item in the Task/KSA Inventory, in terms of its validity as a Task/KSA, scope, wording, and completeness.

- The instructors were asked to identify any additional Tasks/KSAs that should be added to the inventory.

- The entire group was asked to discuss the nature and impact of physical fidelity differences between the TTC simulators and those at the NPPs to which the RIs were assigned or on which the OLEs administered operating exams.

- One class of trainees was asked to pre-test the online survey by completing a paper copy of the draft survey addressing the impact of the physical fidelity differences between the NRC TTC training simulator and those at the NPPs to which they were assigned. ${ }^{1}$

- The entire group was asked to discuss the TTC training structure and content and its role in preparing them for their job duties.

The PNNL team used the information and insights gained from the focus group discussions to modify the Task/KSA Inventory and revise the online survey instrument.

The team made an audio recording of portions of these discussions. A transcript of portions of these discussions is included in Appendix A.

\subsubsection{Discussions with NRC Inspectors and OLEs to Validate Task/KSA Inventory and Its Applicability to New Reactor Designs}

Three members of the PNNL team attended the Pittsburgh workshop on new reactors to discuss Gen III and Gen III+ CRs and simulators with NRC RIs and OLEs. Participants in this workshop had the opportunity to visit a CR simulator and participate in discussions with the NRC staff about issues the highly digital CR might raise for inspectors. Presentations and discussions at the workshop focused on RI and OLE roles and responsibilities for the Gen III and Gen III+ plants and the challenges they posed to regulatory staff. The project team also discussed the applicability of the Task/KSA Inventory items

\footnotetext{
${ }^{1}$ The pretest required only minor revisions in the questionnaire. For this reason, the pretest responses were considered valid and were included as completed questionnaires in the analysis of online survey results.
} 
developed for current generation plants to Gen III/III+ plants and whether the PNNL-developed inventory captured all the Task/KSAs needed for the new generation reactors. After viewing the CR simulator, the discussion focused on how RIs and OLEs might be affected by the extensive use of digital I\&C in the CR and simulators.

\subsection{Priority of Task/KSA Items for Training}

\subsubsection{DIF Analysis}

In the next step of the analysis of learning needs, PNNL applied a method, appropriately called difficulty-importance-frequency, or DIF, analysis to the Task/KSA Inventory items. As discussed in Section 2.6.2, DIF analyses are frequently used in curriculum development to prioritize Tasks/KSAs for training. An additional benefit of a DIF analysis is a more thorough understanding of Task/KSA importance to job performance. This was useful in the assessment of the role and importance of simulators in TTC training of RIs and OLEs.

It also revealed that several Task/KSA Inventory items combined elements that had very different difficulty, importance, or frequency attributes and that therefore needed to be disaggregated into subitems. In conducting this disaggregation, the project team retained the general activity statement of the Task/KSA Inventory item, but reframed it so that the DIF analysis could focus on the specific instance in which it would occur (e.g., normal procedures, emergency operating procedures (EOPs), abnormal operating procedures (AOPs), emergency plan, etc.). This process increased the number of items in the Task/KSA Inventory for the DIF analysis to 61. The final Task/KSA Inventory reflects these modifications.

The project team developed a 5-point rating scale for each of the three following three dimensions:

- difficulty to perform (in job)

- importance to safety

- frequency of occurrence in the position.

The rating scale is shown in Table 3.1. The rating protocol is shown in Appendix B. The team then identified four qualified PNNL staff (former OLEs) and four qualified NRC staff members (current or former RIs) to serve as expert panels to conduct the DIF analysis for the two positions, respectively. The team provided each expert panel with the rating and recording protocol and discussed the process with them. The expert panels conducted the rating as a consensus process, where the panel as a whole reached agreement on the ratings to be assigned.

To establish the priority training rating of each Task/KSA Inventory item, PNNL calculated the Euclidean length of all the possible combinations of DIF ratings ( $5 * 5 * 5 *$ combinations), scaled the lengths as described in Chapter 2, rounded the lengths to integers, and assigned the integer corresponding to the score pattern to the item. The results of the DIF analysis are discussed in Section 6.3. The complete DIF analysis data are presented in Appendix B. 


\subsubsection{Difficulty of Learning and the Importance of Using a Simulator to Learn the Task/KSA Inventory Items}

The project team asked the same two expert panels conducting the DIF analysis ratings to also rate each Task/KSA on the difficulty of learning the knowledge, skills, and abilities necessary to competently perform the task using a 5 -point scale $(1=$ very low to $5=$ very high). They also asked them to use the same process to rate each Task/KSA on the necessity of using a simulator for learning to perform the task competently. For knowledge items, they rated the necessity of using a simulator to learn the knowledge.

Table 3.1. DIF Criteria for Rating the Task/Inventory Items

\begin{tabular}{|c|c|c|}
\hline \multirow[b]{2}{*}{ DIF Dimension } & \multicolumn{2}{|c|}{ Scale Definitions } \\
\hline & RI & OLE \\
\hline $\begin{array}{l}\text { Difficulty of task performance in } \\
\text { current job assignment } \\
\text { Knowledge items: Difficulty of } \\
\text { recalling and applying the } \\
\text { knowledge in current job } \\
\text { assignment }\end{array}$ & $\begin{array}{l}\begin{array}{l}1=\text { Very Low } \\
\text { to }\end{array} \\
5=\text { Very High }\end{array}$ & $\begin{array}{l}1=\text { Very Low } \\
\text { to } \\
5=\text { Very High }\end{array}$ \\
\hline $\begin{array}{l}\text { Importance of task to safety } \\
\text { Knowledge items: Importance of } \\
\text { knowledge to safety }\end{array}$ & $\begin{array}{c}1=\text { Very Low } \\
\text { to } \\
5=\text { Very High }\end{array}$ & $\begin{array}{c}1=\text { Very Low } \\
\text { to } \\
5=\text { Very High }\end{array}$ \\
\hline $\begin{array}{l}\text { Maximum frequency of } \\
\text { performing task in current job } \\
\text { assignment } \\
\text { Knowledge items: Maximum } \\
\text { frequency knowledge is used in } \\
\text { current job assignment }\end{array}$ & $\begin{array}{l}1=\text { Once a year or less } \\
2=\text { Multiple times a year } \\
3=\text { Multiple times a month } \\
4=\text { Multiple times a week } \\
5=\text { Multiple times a day }\end{array}$ & $\begin{array}{l}1=\text { Seldom if ever evaluated } \\
2=\text { Evaluated in some but not al } \\
\text { simulator exams } \\
3=\text { Evaluated at least once in } \\
\text { every simulator exam } \\
4=\text { Evaluated in most scenarios } \\
\text { of every exam } \\
5=\text { Evaluated in all scenarios of } \\
\text { every exam }\end{array}$ \\
\hline
\end{tabular}

\subsection{The Type and Level of Knowledge, Skill, and Ability Needed to Perform the Task/KSA Inventory Items}

To better understand the training needs of RIs and OLEs, the PNNL team next convened an expert panel to develop and apply a framework of knowledge, skill, and ability types and levels to the Task/KSA Inventory items. The expert panel characterized the range and distribution of the types and levels of knowledges, skills, and abilities that RIs and OLEs would need to learn in order to demonstrate CR regulatory competence. The literature on taxonomies reviewed in Chapter 2, particularly Anderson and Krathwohl's (2001) taxonomy, provided the framework for this analysis. The expert panel developed a scale for rating each Task/KSA Inventory item in terms of the level of KSA the item demands. The framework for this rating is shown in Table 3.2; the rating protocol, adapted for applicability to these 
items, is shown in Appendix C. To illustrate, the ratings for levels of internal knowledge ranged from "no recollection or recognition of facts needed..." to "essential to recall or recognize basic facts from memory quickly."

Table 3.2. Framework of Types and Levels of KSAs

\begin{tabular}{|c|c|c|c|c|c|c|}
\hline \multirow{2}{*}{$\begin{array}{l}\text { Knowledge } \\
\text { Needed }\end{array}$} & \multicolumn{6}{|c|}{ Level } \\
\hline & Remember & Understand & Apply & Analyze & Evaluate & Create \\
\hline \multicolumn{7}{|l|}{$\begin{array}{l}\text { Internal } \\
\text { Knowledge of } \\
\text { Facts }\end{array}$} \\
\hline \multicolumn{7}{|l|}{$\begin{array}{l}\text { External } \\
\text { Information } \\
\text { about Facts }\end{array}$} \\
\hline \multicolumn{7}{|l|}{ Conceptual } \\
\hline \multicolumn{7}{|l|}{ Procedural } \\
\hline \multirow[b]{2}{*}{ Skills Needed } & \multicolumn{6}{|c|}{ Level } \\
\hline & & Controlled & & & Automatic & \\
\hline \multicolumn{7}{|l|}{$\begin{array}{l}\text { Learned } \\
\text { psychomotor } \\
\text { acts }\end{array}$} \\
\hline \multicolumn{7}{|l|}{$\begin{array}{l}\text { Manual } \\
\text { movement }\end{array}$} \\
\hline \multicolumn{7}{|l|}{$\begin{array}{l}\text { Visual } \\
\text { perception }\end{array}$} \\
\hline \multicolumn{7}{|l|}{$\begin{array}{l}\text { Auditory } \\
\text { perception }\end{array}$} \\
\hline \multicolumn{7}{|l|}{$\begin{array}{l}\text { Tactile } \\
\text { perception }\end{array}$} \\
\hline \multirow{2}{*}{$\begin{array}{l}\text { Abilities } \\
\text { Needed }\end{array}$} & \multicolumn{6}{|c|}{ Level } \\
\hline & Basic F & iiliarity & & ncy & & \\
\hline $\begin{array}{l}\text { Non-knowledge } \\
\text { abilities } \\
\text { identified in the } \\
\text { inventory }\end{array}$ & & & & & & \\
\hline
\end{tabular}

In addition, the team realized that five of the Task/KSA Inventory items (K1, K2, K3, K6, and K7) represented knowledge about the plant rather than about operator CR behavior, and were therefore better addressed as a type of knowledge against which the remainder of the Task/KSA Inventory items should be rated. The rating form is presented in Appendix C. An expert panel with individuals experienced as RIs and OLEs discussed each Task/KSA Inventory item and assigned a consensus level rating for each Task/KSA Inventory item. Because the ratings for RIs and OLEs were the same, the ratings presented in Appendix C represent the results for both RIs and OLEs. The results are discussed in Section 6.4. 


\subsection{Role of Simulators and CR HSI Systems in Performing and Learning Task/KSA Inventory Items}

\subsubsection{Dimensions of Physical Fidelity: CR HSI Systems and System Elements}

The PNNL team drew upon the human factors and psychological literature, NUREG-0700, Rev. 2, and the personal experience of team members with CR systems and simulators to develop a framework for describing and categorizing the HSI systems and elements of current and advanced reactor CRs and simulators. The team then used this framework to define the information needed to describe and compare the CR HSI systems and simulators of the existing generation of reactor with those of the new reactors. This analysis identified the following eight CR HSI systems:

- procedures

- instrumentation

- controls

- dedicated safety-related controls and displays

- displays of integrated information

- alarms/annunciators

- group-view displays

- workplace design.

A comparison between this taxonomy of systems and those delineated in NUREG-0700, Rev. 2 is shown in Table 3.3. The NRC expects that the taxonomy in NUREG-0700, Rev. 2 is pertinent to both current and advanced reactor CR HSI systems. ${ }^{1}$

Table 3.3. Comparison of the Project and NUREG-0700, Rev. 2 System Taxonomies

\begin{tabular}{|l|l|}
\hline \multicolumn{1}{|c|}{ Project HSI System Taxonomy } & \multicolumn{1}{c|}{ NUREG 0700 Rev. 2 System Taxonomy } \\
\hline Procedure System & Computer-Based Procedure System \\
\hline Instrumentation System & $\begin{array}{l}\text { Parts of instrumentation are in any system that involves } \\
\text { information display }\end{array}$ \\
\hline Control Systems & Soft Control System \\
\hline Dedicated Safety-related Controls \& Displays & Safety Function and Parameter Monitoring System \\
\hline Alarms/Annunciator System & Alarm System \\
\hline
\end{tabular}

The team noted that information displays can potentially be part of a number of systems, e.g., alarm systems, safety functions and parameter monitoring systems, group-view display systems, soft control

\footnotetext{
${ }^{1}$ NUREG-0700, Rev. 2 also includes Computerized Operator Support Systems. These were not included in the taxonomy for this project because it is unclear what operator functions such systems will support. In addition, Communication Systems, also included in the NUREG-0700, Rev. 2 taxonomy, are omitted from the project taxonomy of systems because they were not considered to contribute to RI or OLE task performance.
} 
systems, computer-based procedure systems (as well as the computerized operator support system included in the NUREG-0700, Rev. 2 taxonomy). This was taken into consideration when rating the importance of the system to a Task/KSA Inventory item.

\subsubsection{The Importance of CR HSI Systems and Simulator Dimensions for Each Task/KSA Inventory Item}

To understand which of the HSI systems are most important in RI and OLE training and job performance, an expert panel rated the importance of each of the eight CR HSI systems (described in Section 3.2.3.2 above) to each of the Task/KSA Inventory items. The expert panel consisted of three PNNL staff who had previously been certified as operator licensing examiners and one consultant trainer who presently administers training on the Lungmen ABWR simulator. Two members of the expert panel had also previously worked in the CRs of U.S. reactor plants, and one had been a CR shift supervisor.

The four raters discussed each Task/KSA item and concluded that five of the knowledge items (K1, $\mathrm{K} 2, \mathrm{~K} 3, \mathrm{~K} 6$, and K7) should be omitted from the HSI systems analysis because they did not address the CR specifically, but the plant as a whole. In addition, they divided item RI-Ops-4 into two components. For each of the resulting 51 Task/KSA items, the expert panel developed a consensus judgment regarding the importance of each of the eight HSI systems to learning and performing the item, assigning a value to that system (high importance $=3$, medium $=2$, low $=1$ ) for the item. The resulting ratings are presented in Appendix D. The team analyzed the results of this rating process by examining the histograms of ratings by Task/KSA item (the number of systems receiving a rating of high, medium, and low) and by system (the number of Task/KSA items for which it was rated high, medium, and low).

\subsection{Online Survey of Current RIs and OLEs}

To conduct the next step in the training needs analysis, the PNNL team designed and implemented an online survey of current NRC RIs and OLEs, using the CR Task/KSA Inventory and taxonomy as a central element. Paper versions of the on-line surveys, one for RIs and one for OLEs, is included in Appendix E. The principal information gathering objectives of the online survey were to elicit RI and OLE opinion regarding the following:

- the physical fidelity of various aspects of the CR simulators used for training at the TTC at the time of the survey compared to the specific CRs and CR simulators at their assigned reactors

- the impacts of the fidelity of the TTC simulator on their ability to perform their job duties and to learn course lessons

- whether or not physical fidelity differences between the CRs at their assignments and those at the TTC training simulators were addressed during training and, if so, how the differences were addressed

- the value of the current TTC simulator training to the development of regulatory skills, knowledge of plant operations, and knowledge or operator roles.

The survey also collected demographic information about the respondents' experience in their current assignments and related, previous positions, the types of TTC training they had already received, and their familiarity with various technologies expected to be used in advanced reactor CRs. 


\subsubsection{Questionnaire Design and Pretesting}

In developing the questionnaire, the PNNL team drew upon the literature review and consultation with staff who had taken the TTC training and had experience as NRC OLEs.

The first section of the questionnaire asked respondents to rate the degree to which the TTC training simulator differed in physical fidelity from the corresponding plants or site-specific simulators to which they were or had recently been assigned. It also asked respondents to rate the impact these differences had on both the difficulty of the training and their performance on the job. The second section asked respondents to indicate how their TTC training had addressed differences in physical fidelity between the training simulator and CR at the plant(s) to which the students would be assigned as well as their assessment of that training experience. The third section asked respondents about their overall satisfaction with the TTC training. The final section obtained demographic and experience information about the respondents. Respondents were asked to describe their experience in their present assignment, previous experience, and reactor types used. They were also asked to describe their familiarity with new CR HSI technologies including graphical and group-view displays, soft control systems, computerized procedure and operator support systems, and electronic messaging.

The survey included specific questions examining the differences between the TTC training simulator and the CR panels, controllers, instrumentation, procedures, environment, and alarms of the plant at which the respondent had most recently been assigned. The appraisal of TTC training focused on the value of training by asking for responses ranging from "essential to job performance" to "counterproductive," and requested the respondent to describe counterproductive or unnecessary training aspects.

The survey instrument was reviewed for clarity, flow, and ease of completion by NRC staff and PNNL staff not otherwise working on the project. Feedback from this review was incorporated into the survey instrument. A paper version of the survey was then pre-tested by 14 students attending simulator training at the TTC, confirming that the survey instrument was understandable, elicited responses appropriately, and could be completed within a reasonable amount of time.

\subsubsection{Survey Distribution, Data Collection, and Analysis of Results}

PNNL posted the survey to a website for completion online and, on June 25, 2008, with the assistance of the NRC project manager, the region office sent instructions to RIs and OLEs for accessing the survey. Respondents were asked to complete the on-line survey by July 28, 2008, though responses were accepted through August 5, 2008. The on-line survey was distributed to all current NRC RIs and OLE. PPNL staff periodically checked questionnaires submitted by the respondents to confirm that problems were not encountered in completing the questionnaire. Ninety-seven respondents submitted completed questionnaires: 74 current RIs and 23 current OLEs. A copy of the on-line survey, survey results, and a summary of the characteristics of the respondents will be submitted to the NRC as a CD to enable specific analysis, should that be desired. Responses to the survey were collected and stored locally at PNNL's site and converted to a format amenable to analysis using various software tools. The project team examined the survey data for responses with large variation between respondents, particularly variation that could not be readily explained, as part of its quality assurance process. No such unexpected large response variations were found. 


\subsection{Advanced Reactor Control Room Characteristics and Differences/Similarities with Existing Control Rooms}

\subsubsection{Scope and Approach}

In order to answer the questions, "What are the properties of new reactor CRs (i.e., what will be the targets to be simulated)?" and "What approaches are available for new reactor simulators?", the PNNL team assembled and analyzed the best available design information for the five reactor designs for which the NRC expects license applications in the United States:

- ABWR (advanced boiling water reactor)

- APWR (advanced pressurized water reactor)

- AP 1000 (advanced passive 1000)

- ESBWR

- EPR.

The team assembled information found in design control document (DCD) and combined construction and operating license (COL) applications to the NRC, publicly available internal NRC documentation, publicly available literature, as well as insight from subject matter experts. Based on the extent and quality of this information, the team focused its detailed review on the ABWR and APWR. The steps in this process are illustrated in Figure 3.3. Delays in receiving the KA industry catalogs for the new highly integrated CRs (HICRs) limited the information available for this review.

\subsubsection{Attributes Evaluated and Described}

The team evaluated the different ways to categorize the CR HSI systems, given the different purposes and activities of the study. Table 3.4 presents the characterizations used in different aspects of the project, and how the elements relate to one another.

PNNL structured the review to identify new training simulator configurations and the technologies and arrangements new training simulators are likely to present. In particular, the PNNL team used this information to summarize the HSIs of the new reactor CRs and the range of approaches to physical fidelity likely to be represented in the training simulators as a basis for assessing the suitability of these approaches to teach CR-specific Task/KSAs. The team also reviewed available information on alternative approaches, such as desktop simulations and larger digital simulators, to identify and evaluate the range of feasible simulator fidelity alternatives that might be available to the NRC. The documents reviewed are included in the bibliography. 


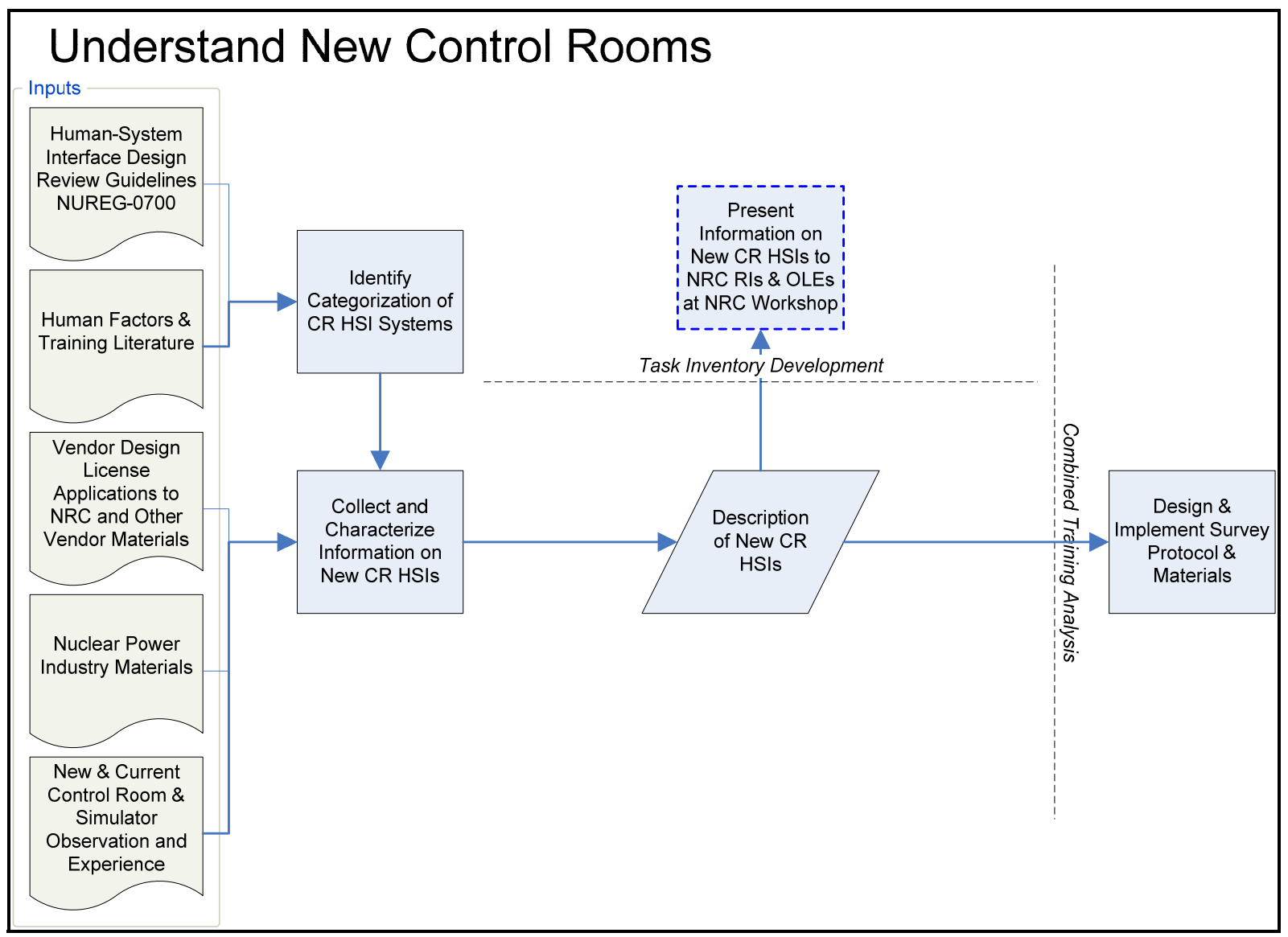

Figure 3.3. Steps in Characterizing and Comparing Control Rooms of New Reactor Designs

Table 3.4. CR HSI System Characterization Strategies

\begin{tabular}{|l|l|l|}
\hline $\begin{array}{c}\text { Analysis of Information on } \\
\text { Advanced Reactors: } \\
\text { "Elements of Control } \\
\text { Room Fidelity" }\end{array}$ & $\begin{array}{c}\text { HSI Systems Used in the Online } \\
\text { Survey (current designs) }\end{array}$ & $\begin{array}{l}\text { HSI Systems Used in Evaluating Use } \\
\text { of HSI System Elements (all designs) }\end{array}$ \\
\hline Displays & Instrumentation; Computer Displays & $\begin{array}{l}\text { Instrumentation System; Dedicated } \\
\text { Safety-related Controls \& Display; } \\
\text { Displays of Integrated Information; } \\
\text { Group-view Display Systems }\end{array}$ \\
\hline Operator Input Devices & Controllers and Switches & $\begin{array}{l}\text { Control Systems; Dedicated Safety- } \\
\text { related Controls \& Display }\end{array}$ \\
\hline Alarms & Alarms/Annunciators & Alarms/Annunciator System \\
\hline Decision Aides & & Workplace Design \\
\hline Communication Equipment & & Procedure System \\
\hline Workplace Design & Panels; Control Room Environment & \\
\hline & Procedures & \\
\hline
\end{tabular}




\subsection{Review of NRC Training Policies, Constraints, and Workforce Characteristics}

To characterize the training parameters that might affect the fidelity requirements of RI and OLE training, the PNNL team reviewed NRC available training and staff development materials. In general, the team was attempting to answer the following questions:

- What are the NRC training requirements for RIs and OLEs?

- Who takes the TTC training? What are the demographic characteristics of the RI and OLE trainees, including previous experience with nuclear reactors?

- What prerequisites are required before the RI or OLE starts this training?

- Are these training requirements stable, or rapidly changing? If they are changing, what aspects are changing and why?

- How is the training for RIs and OLEs structured in terms of training modules and type of training (self-study, face-to-face classroom instruction; training on simulator, etc)?

- Where does the training occur?

- How is the training curriculum organized? What are the learning objectives, especially of the simulator training course?

- What supplementary activities contribute to RI and OLE learning?

- What portion of the preparation for certification as RI and OLE is provided by training (vs. for example, on the job training, previous experience, etc.)?

- Who are the instructors?

- What is NRC policy about training for RIs and OLEs for the new generation of power reactors?

The PNNL team included inquiries about the NRC training policies, constraints, and workforce characteristics in the focus group discussions, described above, and reviewed available documentation of NRC TTC training parameters pertinent to the training of RIs and OLEs including:

- policy statements (e.g., training requirements, NRC Commission Paper SECY-08-0096)

- requirement specifications (e.g., Inspection Manual 1245; Training and Qualifying Procedures, including NRC 2009-ADM 109

- course descriptions, including learning objectives, curricula, reference materials, etc.)

- student profiles

- TTC facility descriptions.

In addition, the PNNL team reviewed available data characterizing the NRC workforce and expectations of change and drew upon personal experience with the training and on the discussions with trainees, instructors, and other staff at the TTC, described elsewhere. 


\subsection{Analysis and Integration of Results}

Figure 3.4 illustrates the relationship among the different information gathering and analytic elements of the study and how they apply to the overall question of physical fidelity needs of TTC training simulators.

\section{Combined Training Analysis}

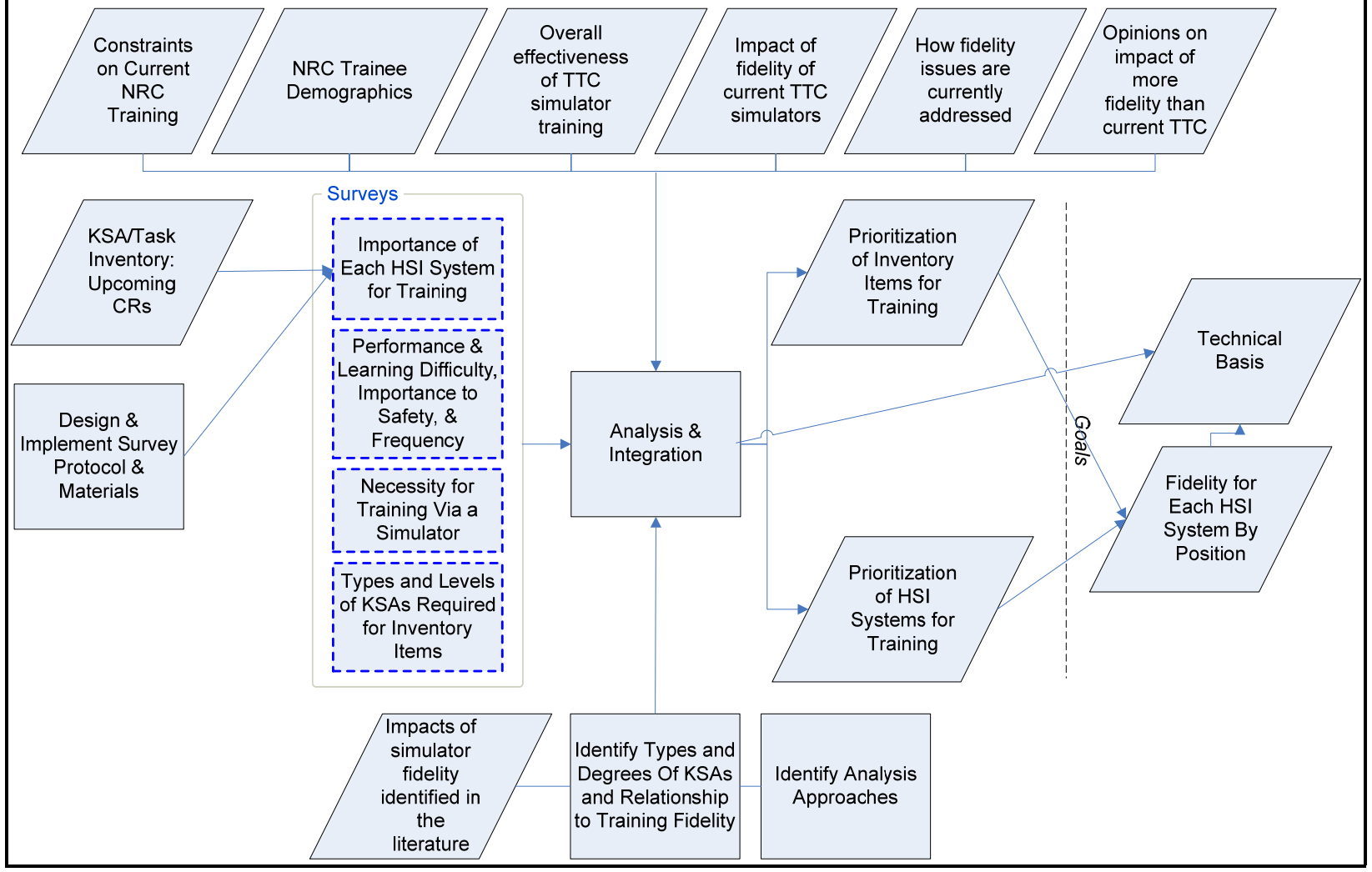

Figure 3.4. How the Study Components Were Integrated: The Combined Training Analysis 


\subsection{Advanced Reactor Control Room Characteristics}

The PNNL team evaluated two main CR HSI designs in some depth for this report - the advanced boiling water reactor (ABWR) $\mathrm{CR}$ and the advanced pressurized water reactor (APWR) CR. The team selected these two designs based on their potential use in the United States and the amount of public information available describing them, along with the availability of operational simulators that demonstrate the HSI design for these CRs. Members of the team were able to observe these simulators and used this opportunity to validate information from the design documents.

The lack of availability of final CR designs and simulators for other new reactor designs has delayed the applicants' completion of the task analyses they must conduct as part of their Human Factors Engineering Program, and which would have served as the basis for identifying specific KAs for operators of those designs. This, in turn, has delayed the availability of the KA Catalogs the NRC will use to update the RI and OLE training. The nuclear industry has indicated that it now plans to provide the new KAs to the NRC to "permit publication of the catalogs in 2011" (NRC 2008 [SECY-08-0096]; NWI 2008).

The new NPPs that are being built in the throughout the world and planned for the United States use a highly integrated, digital CR (HICR) design. This allows for a much different approach to information display and controls (the HSI) than is currently employed in the operating NPP CRs found in the United States today.

Based on review of NRC requirements, applicant materials, and personal experience and observation, the PNNL team concluded that the HSI systems that exist in current next-generation NPP design documents would also exist in the completed designs, although their specific characteristics may differ.

\subsection{Overview of Major Features of Advanced Reactor Control Room Designs}

Two main CR HSI designs were evaluated in some depth for this report- the ABWR CR and the APWR CR. These two were selected because of the amount of public information available describing the designs, and simulators that demonstrate the HSI design are operational.

The significant differences between the current HSI used in CRs, and those used in the main CRs of advanced reactors relates almost entirely to the use of digital circuitry. This enables a level of flexibility that was not previously possible.

\subsubsection{Panel, Workstation, and Workplace Design}

Panels are no longer restricted by the need to hold a minimum number of controllers, indicators and recording devices necessary to operate the plant. Some of the new designs have moved away from the large, fixed-panel concept and use a number of workstations. Other plants maintain large, fixed panels to contain visual display units (VDUs) and fixed displays. All designs use a number of VDUs in place of hard (conventional) controls. 
Use of VDUs and the absence of fixed control switches that are spatially separated from one another make possible a function-based approach instead of a systems based approach to controlling the plant or a transient. An example of this would be reactor pressure vessel (RPV) water level control in a boiling water reactor. With side-by-side VDUs, a single operator would be able to use the feedwater and condensate system as well as various emergency core-cooling systems to attempt to maintain RPV water level.

\subsubsection{Hard Controllers and Switches}

The use of hard controllers and switches is almost exclusively restricted to use as a backup in the event there is a common mode failure of the computer or digital network that would render the soft controls on the VDUs inoperative. This is true for emergency safety features, and in some cases other major plant equipment such as condensate and feedwater pumps. There are a few special cases of using exclusively hard control switches, especially in the ABWR design, for controls such as the reactor mode switch, manual reactor SCRAM (trip), and the control rod insertion and withdrawal pushbuttons.

\subsubsection{Soft Control Systems}

Widespread use of soft controls that are VDU-based is employed in all designs that have been evaluated. This is a fundamental difference between the new HSI design and the current CR designs.

\subsubsection{Fixed-position Displays}

All designs use some type of fixed-position display of key plant parameters, especially those that are required to satisfy Regulatory Guide 1.97 requirements and for use of emergency operating procedures. In some cases, these fixed-position displays are hard panels, in other cases it is a large screen or projection.

\subsubsection{Hard Displays}

Hard fixed position displays are used in both designs that were evaluated in detail. The displays are used for certain key parameters in the event there is a common mode failure of the computer or digital network that would render the plant computer system driven indications unavailable. In the new designs, these are the exception, rather than the majority of the displays found in the current CRs.

\subsubsection{Single-Function (single-page) Visual Display Unit-based Displays}

There appears to be no significant use of single function VDU-based displays in the new designs.

\subsubsection{Page-Based, Single-operator Displays}

As described above in the CR design with use of VDUs, numerous page-based, single-operator displays are used. 


\subsubsection{Large Displays}

Large displays intended for displaying information that the entire CR staff can view, are essentially a new concept in the CR design. There are examples of limited information that can be displayed for CR viewing with current design CRs, however this is usually limited to a single parameter or a small group of parameters. Using either a fixed display or a variable (i.e., changeable assigned values or other aids) display, the new HSI designs allow for an integrated information display.

\subsubsection{Computerized Operator Support Systems}

The full scope of computerized operator support systems is not finalized yet in many of the new CR designs. Some have the capability for automated plant operation under the control of the licensed operator.

\subsubsection{Displays of Integrated Plant Information}

See discussion on large displays (Section 4.1.8).

\subsubsection{Dedicated Safety-related Controls and Display System}

As partially described in previous sections above, the HSI design of the new CRs employs both VDUs for soft-screen control and display, and hard switches as a backup feature for designated engineered safety features and other key components.

All plant designs evaluated use dedicated safety-related VDUs and dedicated hard switches for safetyrelated controls. Also, there are dedicated safety-related parameters displays. This level of redundancy typically does not exist in current plant CR designs.

\subsubsection{Alarm Display and Processing}

A significant difference in the new plant designs is the minimum number of fixed alarm tiles or displays. Typically, only system level alarms, with individual alarming conditions indicated on a VDU based alarm screen, or dedicated VDU-based alarms, with some type of indication on the display to alert the operator to an alarming condition, are included in new plant designs.

\subsubsection{Control Systems}

The HSI control system designs of the plants evaluated rely primarily on operator interface through the use of touch screen (or mouse-driven) VDUs. There appear to be two major approaches taken: 1) the use of VDUs that allow operation and monitoring of either safety-related and non-safety-related systems and components with backup dedicated safety-related VDUs that are exclusively for the control of safetyrelated systems; or 2) separate safety-related VDUs for control and monitoring of safety-related systems and non-safety-related VDUs for control of non-safety-related systems and components only (with monitoring capability of both non-safety-related and safety-related systems). 
In all cases, there are redundant hard switches for designated engineered safety feature systems in the event of a common mode failure that would render the VDUs inoperative. Current CR designs use a varying amount of computer screens for monitoring only. Typically, there are no redundant means of control other than the installed conventional control devices.

\subsubsection{Instrumentation Systems}

In the current CR designs there are fixed position indications of key plant parameters that are often spatially separated from one another (e.g., normal RPV water level or steam generator water level indications in one location, accident indications in another). These indications are backed up by nonsafety-related computer screens that can typically display a wide range of the available indicators. The number of screens varies considerably among the operating plant main CRs.

The new CR HSI designs use large displays (both fixed and variable) and a number of VDUs that are located throughout the $\mathrm{CR}$, allowing all available ranges or types of relevant information to be viewed by any operator or senior operator for plant control and monitoring.

\subsubsection{Procedure Systems}

Current plant CR designs use hard copies of procedures almost exclusively. This practice requires the operator to locate the correct procedure, retrieve it from the storage area, and find the correct location within the procedure prior to using it. Some plants have a computer-based annunciator response procedure system that can be displayed on a computer screen.

The new CR designs evaluated have the capability to provide a dynamic on-line procedure system. Typically, this involves procedures that can be accessed from a VDU (or certain dedicated procedure VDUs), and have links to the systems being operated on-screen. The full development of these systems is not complete, so only this overview of capabilities is provided.

\subsubsection{Group-view Display Systems}

Current CR design has examples of limited group-view displays, most being contained on a plant computer VDU screen with limited readability by everyone in the CR. Most displayed information is provided by the fixed-position instrumentation that is distributed throughout the $\mathrm{CR}$ on various $\mathrm{CR}$ panels.

The new HSI designs provide specifically for integrated group-view displays, both fixed and variable. The variable display allows viewing of information other than what was chosen for the fixed displays, or in many cases, allows the display of dynamic charts or graphs. These displays are specifically designed for full CR staff viewing.

\subsubsection{Integration of Information Displays and Control}

The current CR designs typically have some instrumentation associated with the system or component controls located in close to the fixed controls. Examples include motor current, pump discharge pressure, and pump or system flow. 
The new HSI designs use schematic representations of the system to be operated on the VDU. Control of the components is performed by touch screen operation (or mouse) on the VDU, and system parameters are displayed on the same VDU. In many cases, side-by-side VDUs can be used to display all available information relating to system or component operation.

\subsection{Overview of the Major Differences Between the ABWR and APWR Main Control Room HSI Technologies}

Analysis of the ABWR and APWR, as discussed in Chapter 3, identified the following significant differences between current and new main CR HIS technologies.

1. Panel, workstation, and workplace design: The ABWR CR uses large, fixed panels to contain VDUs and fixed displays. The APWR design uses one main control console to use VDUs, and a large display panel.

2. Hard controllers and switches: The ABWR CR distributes backup "hard" or conventional control switches on various panels. It also uses the hard switches for certain special functions (e.g., reactor mode switch, and control rod insertion and withdrawal). The APWR design has segregated areas for the hard controls: 1) the Diverse HSI Panel and 2) the System Level Hardwired Switch Area on the Operator Control Console (OCC).

3. Soft control systems: Both designs use touch screen VDUs. The APWR also has provision for operation on screen with a mouse.

4. Fixed-position displays: Both designs have fixed position displays. The ABWR has the display integrated into an operating console; the APWR has just a front display (the large display panel).

5. Hard displays: Both designs have a small group of safety-related hard displays.

6. Single-function (single-page) VDU-based displays: There appears to be no significant use of this made in the new designs.

7. Page-based, single-operator displays: Both designs have multiple VDUs with page based, singleoperator displays for control of systems and components.

8. Large displays: Both designs have large displays, both fixed and variable. The ABWR design has the fixed display as an integral part of an operating console (the wide display panel), and a large variable display on the wide display panel. The APWR has an integral large display panel with both a fixed and variable display section.

9. Computerized operator support systems: The full scope of computerized operator support systems is not finalized yet in many of the new CR designs. Some have the capability for automated plant operation, under the control of the licensed operator.

10. Displays of integrated plant information: See large displays.

11. Dedicated safety-related controls and display system: Both designs have dedicated safety-related VDUs for control and monitoring of safety-related systems. Both designs have dedicated backup hard switches for operation of selected engineered safety feature systems.

12. Alarm display and processing: The ABWR design has fixed position system and plant level alarm tiles. Individual alarming conditions are found on the alarm page that is accessible from any VDU. 
The APWR design has two alarm summary areas on the large display panel. There are alarm VDUs on the operator control console, the supervisor console, and the shift technical advisor (STA) console.

13. Control systems: The ABWR design uses separate VDUs for operation of safety-related systems and non-safety-related systems, each only controlled from their respective type VDU. The APWR allows operation of both safety-related and non-safety-related systems from the non-safety-related operational VDUs, and has dedicated safety-related VDUs for control of the safety-related systems should the operational VDUs fail for whatever reason.

14. Instrumentation systems: Both designs use VDU and display based instrumentation readouts. Both designs have certain safety-related hard fixed position instrumentation displays in the event of a common mode failure of the digital displays.

15. Procedure systems: There is insufficient information to compare the procedure systems.

16. Group-view display systems: Both designs have a group-view display arrangement. The ABWR refers to this as the wide display panel, which includes a fixed display and a large variable display VDU. The APWR has the large display panel which contains both a fixed display and a variable display area.

17. Integration of information displays and control: Both designs integrate system information and controls on VDU displays.

\subsection{APWR Main Control Room}

\subsubsection{Basic Characteristics}

The following list summarizes the basic characteristics and key features of the APWR main CR:

- a single, integrated operator control console

- dedicated function switches (i.e., "conventional switches") for operation of safety systems in the event of common (computer) failure

- non-safety VDUs, which allow monitoring and control of both non-safety and safety functions

- a non-safety large display panel, which provides spatially dedicated continuously visible (SDCV) information important to plant operability and safety

- dedicated safety-related VDUs, which allow monitoring and control of safety functions

- safety-related SDCV conventional controls for system level actuation of reactor trip and engineered safety feature actuation systems

- the ability to monitor and control critical safety functions through systems that are diverse from the HSI and supporting systems described above. 


\subsubsection{Detailed Descriptions of Specific Features of the APWR}

The following four particular areas or features are described below:

- panel, workstation, and workplace design

- HSI technology

- HSI systems

- Integration of information displays and control.

\subsubsection{Panel, Workstation, and Workplace Design: the Physical Location and Layout of Different CR Equipment}

The main CR panels comprise four main panels that house control, display, and alarm equipment. The four panels consist of a console-style OCC where the licensed operators would normally sit, a large display panel that is the main CR group-view display, a console-style supervisor console, and a consolestyle shift technical advisor (STA) console.

Figure 4.1 shows the main CR layout and the relative position of the panels.

The OCC houses the system level hard-wired switch area, alarm VDU, operating procedure VDU, the non-safety-related operational VDUs from which both non-safety-related systems and safety-related systems can be operated and monitored, and the safety VDUs from which only safety-related systems can be operated.

Both safety-related and non-safety-related VDUs contained on the OCC are active touch screen devices for monitoring and control of plant equipment. The VDUs contained on the supervisor console and STA console are for monitoring only.

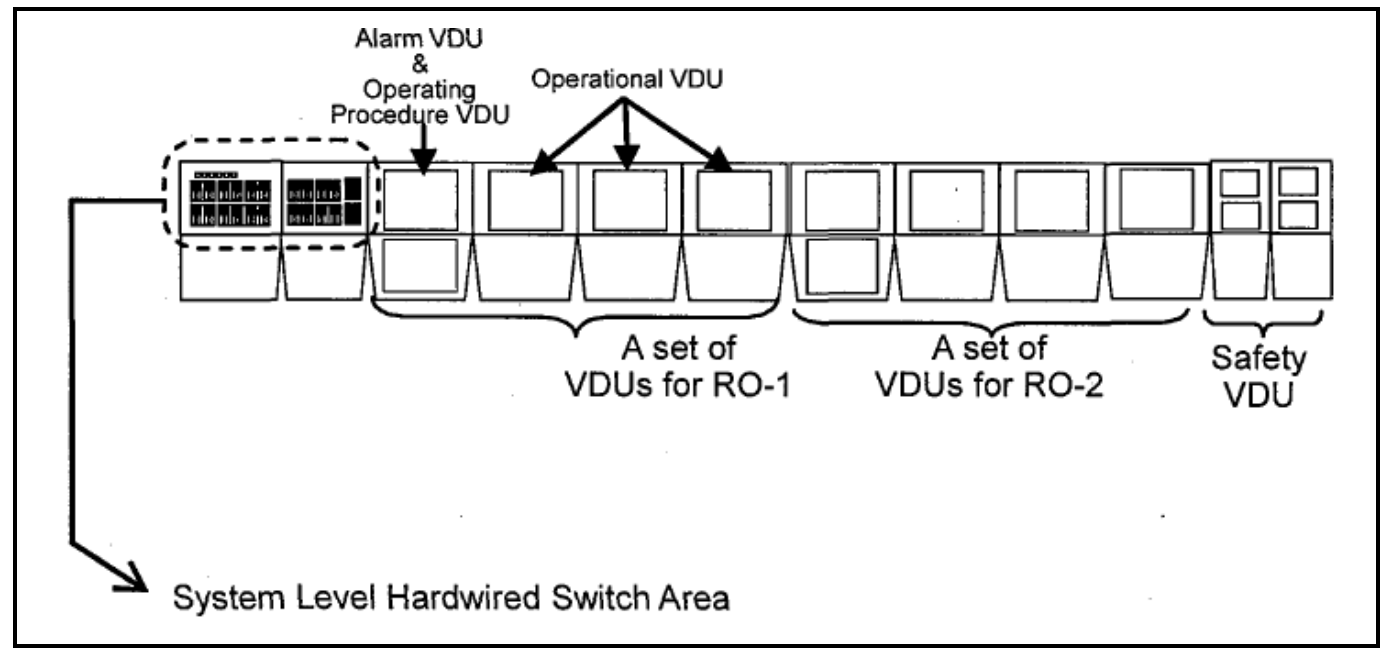

Figure 4.1. Equipment Arrangement of Operator Control Console 


\subsubsection{HSI Technology}

\section{Controls}

Hard Controllers and Switches: System level operation switches to be used by operators in the event of an emergency are provided. The functions are realized by conventional hard-wired Class 1E module switches that accessible to the operator. The functions at the system level are:

- reactor trip

- actuation of emergency core cooling system (ECCS)

- containment vessel isolation phase A

- main steam flow isolation

- emergency feedwater flow isolation

- actuation of emergency feedwater flow

- actuation of containment vessel spray and containment vessel isolation phase B

- main CR heating, ventilation, and air conditioning (HVAC) isolation

- charging water flow isolation

- the diverse HSI panel contains conventional switches and indicators for safety operation (for backup in the event of common cause failure).

Soft Control Systems: Soft control interface is via the touch screen VDUs by either touch screen interaction or use of a mouse. As described above, only the VDUs on the OCC are active control devices.

\section{Information Displays (display devices and user-interaction)}

Fixed-Position Displays (Instrumentation): Fixed position display of key plant parameters and major equipment status is provided on the fixed display area of the large panel display.

- Hard displays (meters, gauges, plotters, etc.) - Safety-related fixed-position indicators are included on the diverse HSI panel. These are to support safety system operation in the event of a common mode failure that would affect VDU operation.

- Single-function (i.e., single-page) VDU-based displays - Insufficient information is available to describe these displays.

- Page-based, single-operator displays (multiple pages or windows; typically VDU-based; can also be projected; also called display networks) - The safety-related and the non-safety-related operational VDUs contain pages for operation safety-related and non-safety-related systems. Control of safetyrelated systems can be performed from the operational VDUs or from the safety-related VDUs, whereas control of non-safety-related systems can be performed from the non-safety-related VDUs.

- Large displays (which can include a mix of fixed position and VDUs with multiple pages) - Large display format is provided by the fixed display area on the large display panel, and by the variable display area, which is also on the large display panel. The contents of the variable display area can be selected from the operator console and from the supervisor console. The variable display area can also automatically display pre-selected screens. 
Computerized Operator Support Systems: Computerized operator support systems are still in the development stage. There is insufficient information or existing technology to describe this system.

\subsubsection{HSI Systems (Implementations Using HSI Technology)}

\section{Displays of Integrated Plant Information (Safety Function and Parameter Monitoring System - includes safety parameter display system [SPDS])}

The large display panel provides real-time plant status information during all phases of plant operation. The large display panel provides an integrated overview of plant conditions and major equipment status. The two functional portions of the large display panel related to integrated plant information display are:

- Fixed display area, which displays the same information at all times, has the following sections:

- Shared alarm summary display (for primary system)

- Plant output parameter summary display

- Shared alarm summary display (for turbine generator and electrical system)

- Variable display area, which is described above in Section 4.1.8.

\section{Dedicated Safety-related Controls and Display Systems}

Dedicated safety VDUs are used to monitor and control the safety-related equipment. The primary control interface is designed to be from the non-safety-related operational VDUs, but the safety VDUs are installed in the event of failure of the operational VDUs.

\section{Alarms/Annunciators}

Alarm Display - The large display panel has grouped alarm displays as described in Section 4.3.1. More detailed information concerning the alarm is available on the Alarm VDUs.

- Alarm processing systems (alarm reduction/filtering, prioritization, integration/derivation) - The grouped alarm displays on the large display panel provide dynamic alarm prioritization, as well as on the alarm VDU displays. This prioritization is event specific, and will display the most important alarms at the time at the highest priority by a color coded scheme.

\section{Control Systems}

Control of plant systems and components is accomplished either through fixed-position conventional control switch devices (found on the system-level hardwired switch area or the diverse HSI panel, or touch screen VDUs.

- The conventional switches on the system-level hardwired switch area and the diverse HSI panel are for defense against a potential common mode failure. Normally, system control is implemented using the touch-sensitive VDUs. 
- Non-safety-related operational VDUs are contained on the OCC for control of both non-safety-related systems and safety-related systems. Normally, control of both categories of systems is to be accomplished with the operational VDUs.

- Safety-related VDUs (physically separate and functionally independent) are installed on the OCC. These VDUs are used for control of individual safety-related systems when the Operational VDUs are not functioning.

\section{Instrumentation Systems}

The large display panel shows signals from accident monitoring instrumentation of all Regulatory Guide 1.97 variable types. In addition, the Protection and Safety Monitoring System (PSMS) information contained on the large display panel shows signals for Type A and B variables.

The group of operational VDU display formats also provides the safety parameter display system (SPDS) functions.

\section{Procedure Systems}

- Procedure information display. Information not currently available.

- Computer-based procedure systems. The operating procedure VDUs provide computer-based operation procedure displays near the operational VDUs and the alarm VDUs. When a procedure is displayed on the operational procedure VDU, a link can be made to relevant display on the operational VDU next to it.

\section{Group-view Display Systems/Displays for Shared Information}

The large display panel has two group-view display systems. The fixed display area and the variable display area discussed above.

\subsubsection{Integration of Information Displays and Control (across displays with different functions; integration of soft controls into mimic screens)}

The non-safety-related operational VDUs and the safety-related VDUs integrate display information and controls on the same screen. Typically, when operating a system, there are one or more screens that contain system diagrams with the system components and key parameters both displayed.

\subsubsection{Illustrations of the APWR Main Control Room}

Figure 4.2 is an overhead view of the APWR main CR. It shows the orientation of the various operator control and monitoring consoles and the display panels. Figure 4.1 shows the equipment arrangement contained on the OCC and Figure 4.3 shows the equipment arrangement on the supervisor console and on the STA console. Figure 4.4 shows the arrangement of the displays on the fixed display area and on the variable display area. 


\subsection{ABWR Main Control Room}

\subsubsection{Basic Characteristics of the ABWR Main Control Room}

The following list summarizes the basic characteristics and key features of the ABWR main CR:

- a single, integrated main control console (MCC)

- non-safety-related VDUs for non-safety system control and monitoring and safety system monitoring

- separate set of on-screen control VDUs for safety system control and monitoring. The operation of this set of VDUs is independent of the plant computer system, and all equipment associated with their functions of safety system control and monitoring are divisionally separate and qualified to standards for safety-related, Class 1E equipment

- dedicated function switches (i.e., "hard switches") on the control consoles

- operator selectable automation of predefined plant operational sequences

- operator selectable semi-automated mode of plant operations, which provide procedural guidance on the control console VDUs

- the capability to conduct all plant operations in an operator manual mode

- wide display panel (WDP) which presents information for use by the entire CR operating staff

- the inclusion on the wide display panel of fixed-position displays of key plant parameters and major equipment status. The fixed-position displays are independent from the plant computer system equipment that performs the calculations for process monitoring functions

- the inclusion in the fixed-position display of environmentally qualified displays.

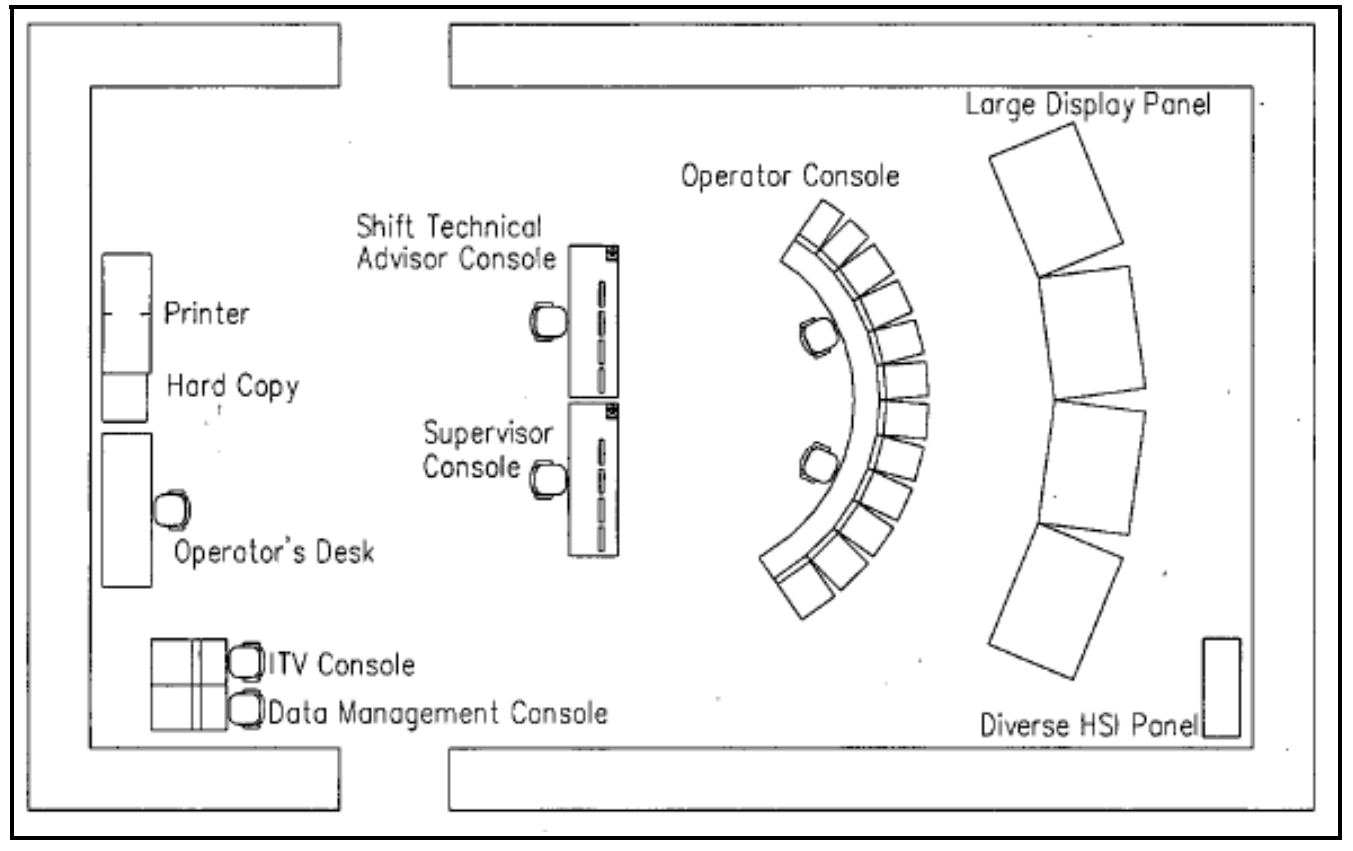

Figure 4.2. Typical Layout of APWR Control Room 


\subsubsection{Detailed Descriptions of Specific Features of the ABWR}

The following nine particular areas or features are described in this section:

- panels

- controllers and switches

- soft control systems

- instrumentation

- alarms/annunciators

- computer displays

- group-view display systems

- procedures

- computerized operator support systems.

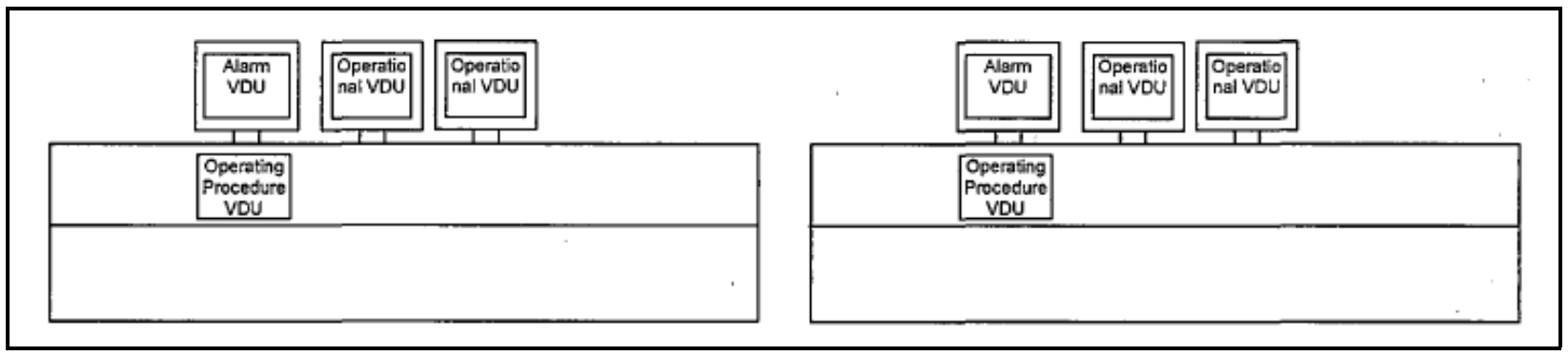

Figure 4.3. Equipment Arrangement of Supervisor Console and STA Console

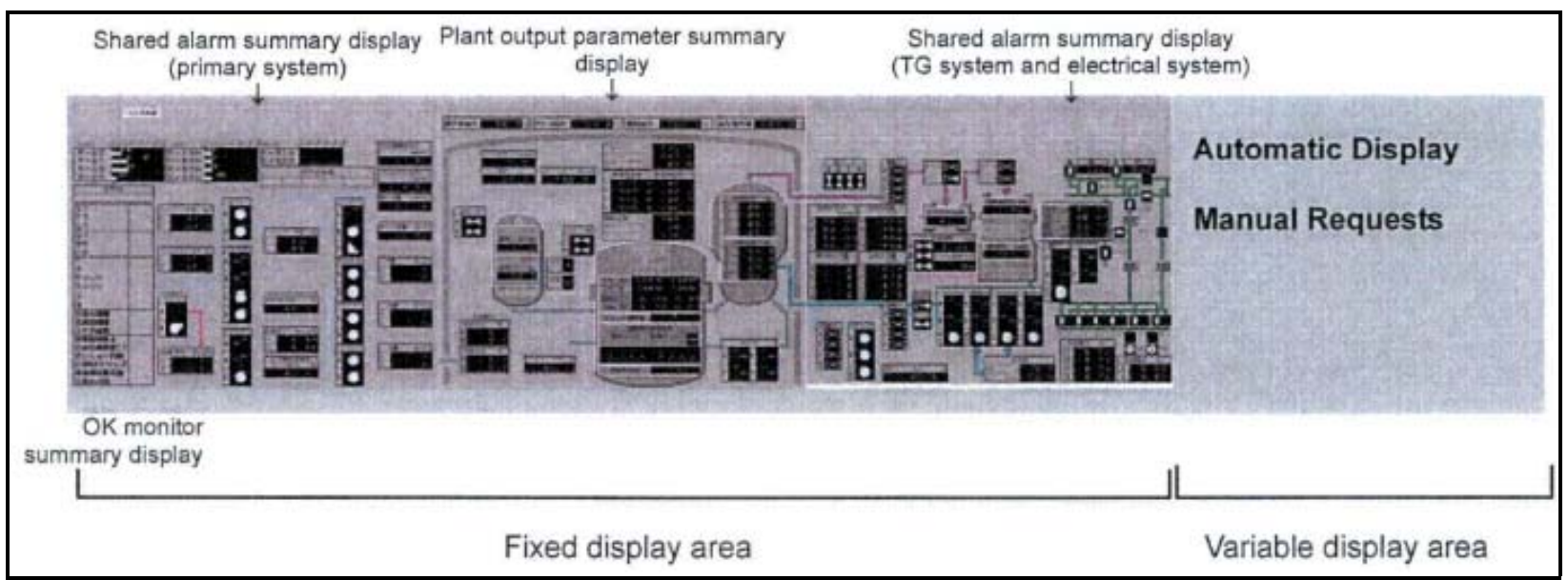

Figure 4.4. Arrangement of Large Display Panel 


\subsubsection{Panels}

The main CR panels are composed of three panels that house control, display, alarm, and communication equipment. The three panels consist of a console-style MCC where the licensed operators would normally sit, a benchboard-style WDP, and a console-style shift supervisor/manager console.

Control, display, alarm, and communication equipment is physically integrated within the main CR panels. The types of equipment that are installed in each panel are VDUs, fixed-position switches, fixedposition displays, fixed-position alarms, large video display units, and communications equipment (voice and alarm generation). Operator tasks requiring control, display, alarm, and communication functions are accomplished using this equipment.

The VDUs display operator information such as operating conditions, alarms, diagnostics, and procedures. They also provide clearly identified locations on screen surfaces that, when touched, allow the operator to perform control actions such as changing a system operating mode or operating major components such as valves and pumps. Fixed-position switches provide operators' access and visual feedback for certain control actions that are more immediate compared to that provided by the VDUs. Fixed-position displays are dedicated to the continuous display of single information items (e.g., one plant parameter). Similarly, fixed-position alarms are indicators dedicated to annunciating single information items concerning plant-level or system-level conditions. The large video display unit allows for group viewing (e.g., alarm summaries, power-to-flow map, or EOP curves) in a practical manner.

\subsubsection{Controllers and Switches}

Some circuits for fixed position controls and displays are individually wired to the WDP and MCC for defense against a potential common mode failure of the safety system logic control (SSLC) or the multiplexing system coincident with certain accident conditions. Special performance requirements make it necessary that some control and display equipment be operationally dedicated to a specific system (e.g., RCIS or RHR). Other than these special cases, there are relatively few plant systems requiring mechanical interfacing of system controls and displays with the main CR panels. Most system controls and displays are implemented using the touch-sensitive VDUs.

Fixed-position, dedicated function switches are used for certain operator control actions. The switches are divisionally separated according to system at fixed locations on the MCC and the WDP. The switches initiate safety systems and control system component functions (e.g., valve open/close).

\subsubsection{Soft Control Systems}

Safety-related VDUs (physically separate and functionally independent of the plant computer system) are installed on the MCC and the WDP. These VDUs are used for control and display of individual safety-related systems when a system component must be operated manually. The VDUs are divisionally separated devices qualified, along with their supporting display processing equipment, to Class $1 \mathrm{E}$ standards. These VDUs are only used for monitoring and control of equipment within a given safety division (that is, there are divisional panels for each of the Class $1 \mathrm{E}$ safety-related divisions). 
Non-safety-related VDUs are installed for the following functions and tasks:

- monitoring of plant systems, both safety and non-safety

- control of non-safety system components

- presentation of system-level and equipment-level alarm, diagnostic and procedure information.

These VDUs, along with their supporting display processing equipment, operate independent of the PCS processors that perform calculations for process monitoring functions. Data collected by the PCS are available for monitoring on these VDUs. All available display formats can be displayed on any of these VDUs.

\subsubsection{Instrumentation}

The available instrumentation in the main CR basically falls into one of the following categories:

- information available on the safety-related and non-safety-related VDUs

- fixed position displays that are incorporated into the WDP

- certain key parameter fixed position displays that are hard wired to the WDP and MCC for defense against a potential common mode failure of the SSLC

- the safety parameter display system (SPDS) function is part of the plant status summary information that is continuously displayed on the fixed-position displays on the WDP.

A subset of the parameters shown on the WDP comprises the minimum set of safety parameters that are necessary for compliance with NUREG-0737, or committed to be fixed-position.

\subsubsection{Alarms/Annunciators}

PCS alarm processing units drive dedicated alarm tiles on the WDP (plant-level alarms, system-level alarms, and alarms for conditions related to emergency operating procedures). The alarms are controlled by alarm processing controller units that operate independently of the PCS processors that perform calculations for process monitoring functions.

Alarm processing units also present non-safety equipment alarm information on non-safety-related VDUs. The PCS performs the function of supervisory processing of plant alarms for operator presentation (alarm filtering and prioritization). The alarms displayed (based on a pre-defined priority) are selectable by the operator.

\subsubsection{Computer Displays}

In addition to the information described above for soft control systems, the following information about computer displays was obtained:

- A large variable display panel that is contained on the WDP. The large variable display is driven by the PCS. Any display image available through the PCS (e.g., images from VDUs at the MCC) can be shown on this large variable display. 
- Display only screens on the SSC. The SSC is a workstation for the main CR shift supervisor/shift manager to monitor the plant. Control of plant processes and plant equipment cannot be performed from the SSC. During any operating mode, the shift supervisor/shift manager can view the same information that is also presented on MCC and WDP VDUs.

\subsubsection{Group-view Display Systems}

The CR is designed with two display systems that are viewable by the entire main CR staff: the large variable display described in Section 5.4.2.6, and the information contained on the WDP.

The WDP is a large benchboard that provides real-time plant status information during all phases of plant operation. The WDP provides an integrated overview of plant conditions and major equipment status. The four functional portions of the WDP related to group display are

- a fixed-position, plant-level alarm display

- a fixed position mimic

- a fixed-position, system-level alarm display

- a large variable display.

The fixed position mimic provides indication for

- ECCS status and parameters

- RPV parameters

- primary containment parameters

- feedwater system parameters

- main steam system parameters

- other safety-related and select non-safety-related information.

\subsubsection{Procedures}

The PCS provides operator aids such as on-line electronic procedures for plant normal, abnormal and emergency operations. Plant alarm response procedures are also provided. A workstation, also part of the PCS and connected to the PCS site computer network, is used for switching and tagging activities.

\subsubsection{Computerized Operator Support Systems}

The PCS has two sub-systems that supply operator support. They are the Performance Monitoring Control System (PMCS) and the Plant Generation Control System (PGCS). 
The PMCS obtains both safety-related and non-safety-related data from the plant-wide multiplexing data network to support all PCS functions:

- monitoring plant conditions

- recording plant data for historical analyses

- performing core nuclear steam supply, equipment monitoring, and plant thermal performance and prediction calculations

- performing alarm processing to support CR alarm annunciation

- providing for updates of all non-safety dynamic display elements in the MCR.

The PGCS is a top-level controller that monitors the plant conditions, issues control commands to non-safety-related systems, and adjusts setpoints of lower level non-safety-related controllers. PGCS supports the automation of the plant startup, power range, and normal shutdown operations. The operator interfaces with PGCS through a series of breakpoint controls to initiate automated sequences. The PGS has three levels or modes of operation:

- Automatic. In automatic, the PGCS issues commands to system-level controllers. Command signals for setpoint adjustments of lower-level controllers and for startup/shutdown of other systems are executed by PGCS with the operators' execution commands. Operator interfaces with PGCS through a series of breakpoint controls to initiate automated sequences.

- Semi-automatic. In semi-automatic, the PGCS monitors progression of plant operations. It automatically provides prompts and guidance to operator (procedural steps displayed on screen).

- Manual. In manual, no operator guidance or prompts are provided by PGCS.

\subsubsection{Illustrations of the ABWR Main Control Room}

Figure 4.5 shows the ABWR CR at the Lungmen plant. Figure 4.6 shows the wide display panel at Lungmen, with the fixed-display (Mimic) information highlighted. Figure 4.7 shows the ABWR CR wide display panel, with the large variable display highlighted.

\subsection{Implications for Physical Fidelity of TTC Simulators in Advanced Reactor Training}

The CR designs for Gen III/Gen III+ reactors evaluated for this study ${ }^{1}$ use more "soft" displays and controls than current CRs, making the HSI technology in the new reactor CRs and simulators very different from those currently in use. However, the systems to be controlled, and the parameters that have to be monitored to verify safe operation are essentially the same in the advanced as in the current CRs. Some of the new designs, such as the Advanced Passive (AP)-600 and the AP-1000, place greater reliance on passive heat removal systems, with consequent modification of system parameters. However, details about the CR design for these plants were not available at the time this report was written. The basic reactor design of the other advanced reactors is not different enough from current designs to require

\footnotetext{
${ }^{1}$ Information about the passive design and its heat removal system as described by the World Nuclear Association is available at http://world-nuclear.org/info/inf08.html.
} 
different control parameters. Because of the screen size, the jobs of the RI and OLE will be somewhat complicated because it will be harder for them to see, from a distance, what the operator is observing and what soft controls they are manipulating. Many control and indication systems that are important to safety will be dedicated displays or switches similar to those in current use.

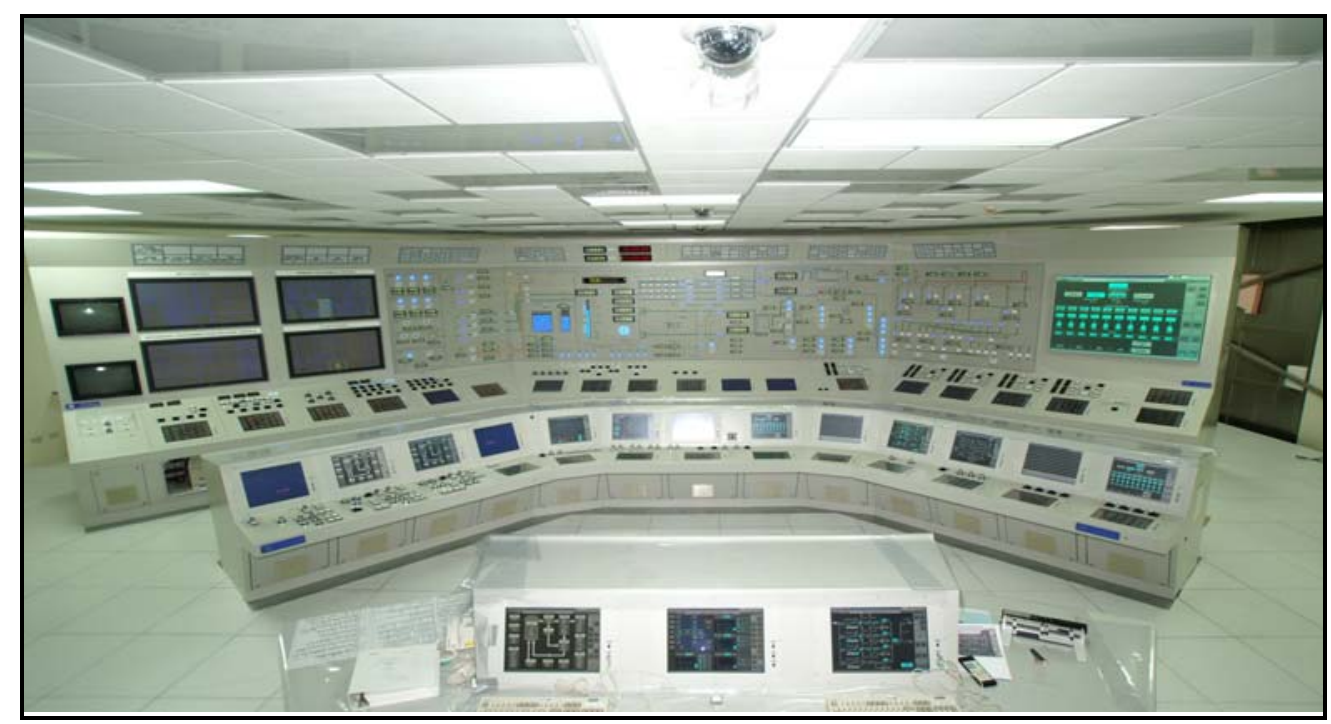

Figure 4.5. ABWR Control Room (Lungmen)

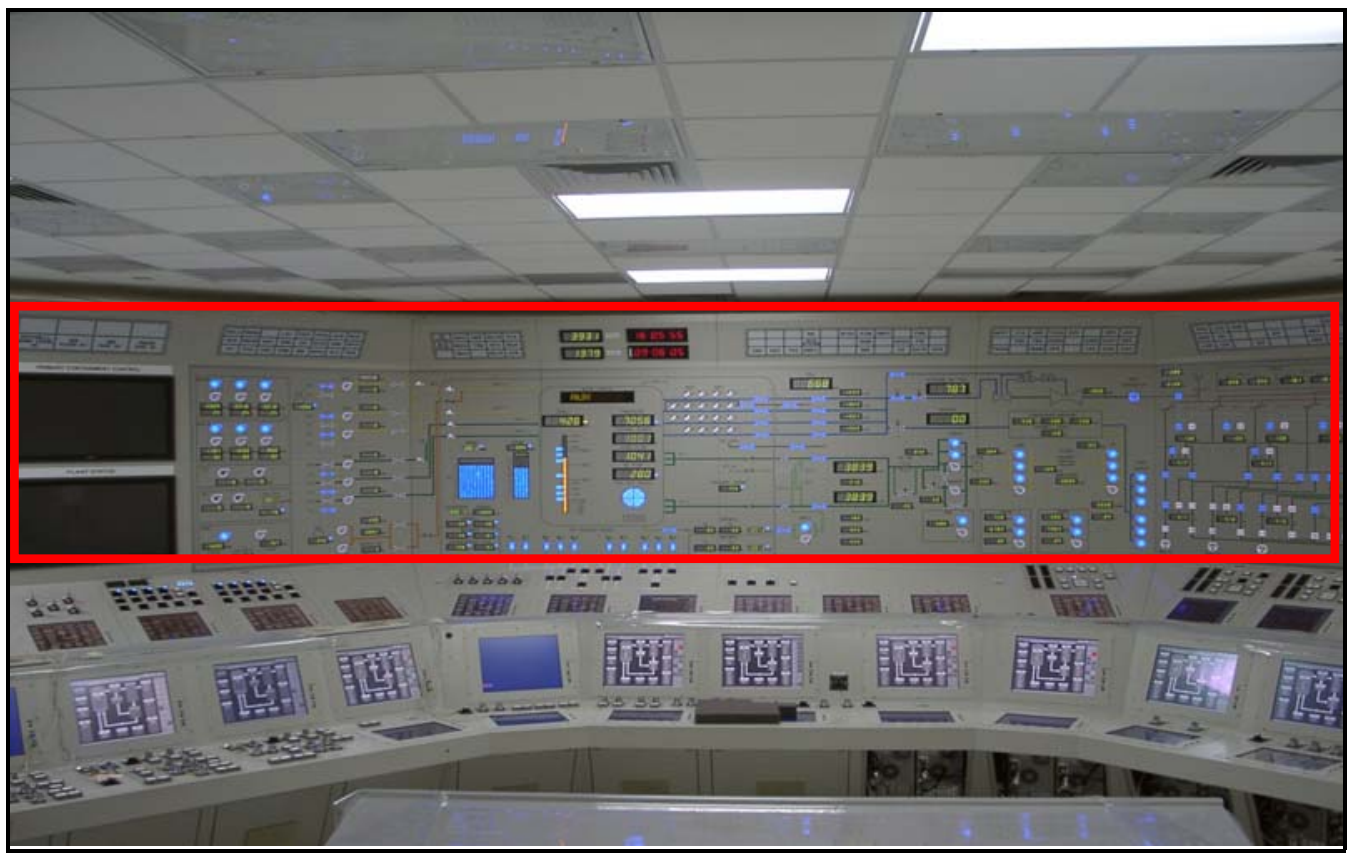

Figure 4.6. ABWR Control Room (Lungmen) Wide Display Panel Highlighting Fixed-Display (Mimic) Information

The team's analysis indicates of available information about advanced reactors' CRs and simulators is that, to a large extent, the Tasks/KSAs currently taught at TTC will apply to the new reactors in the same 
way they apply to existing power plants. The overall licensing conditions of these Gen III/Gen III+ plants are expected to be the same as current reactors. In addition, the regulatory goals of the RI and OLE are expected to be the same for the advanced reactors as existing ones. Consequently, the level of fidelity provided by the current TTC simulators is expected to be sufficient to train RIs and OLEs for jobs at advanced reactors - the simulator needs to represent a typical reactor CR of each advanced reactor design, but it does not need to represent the particular plant at which the RI or OLE candidate will work.

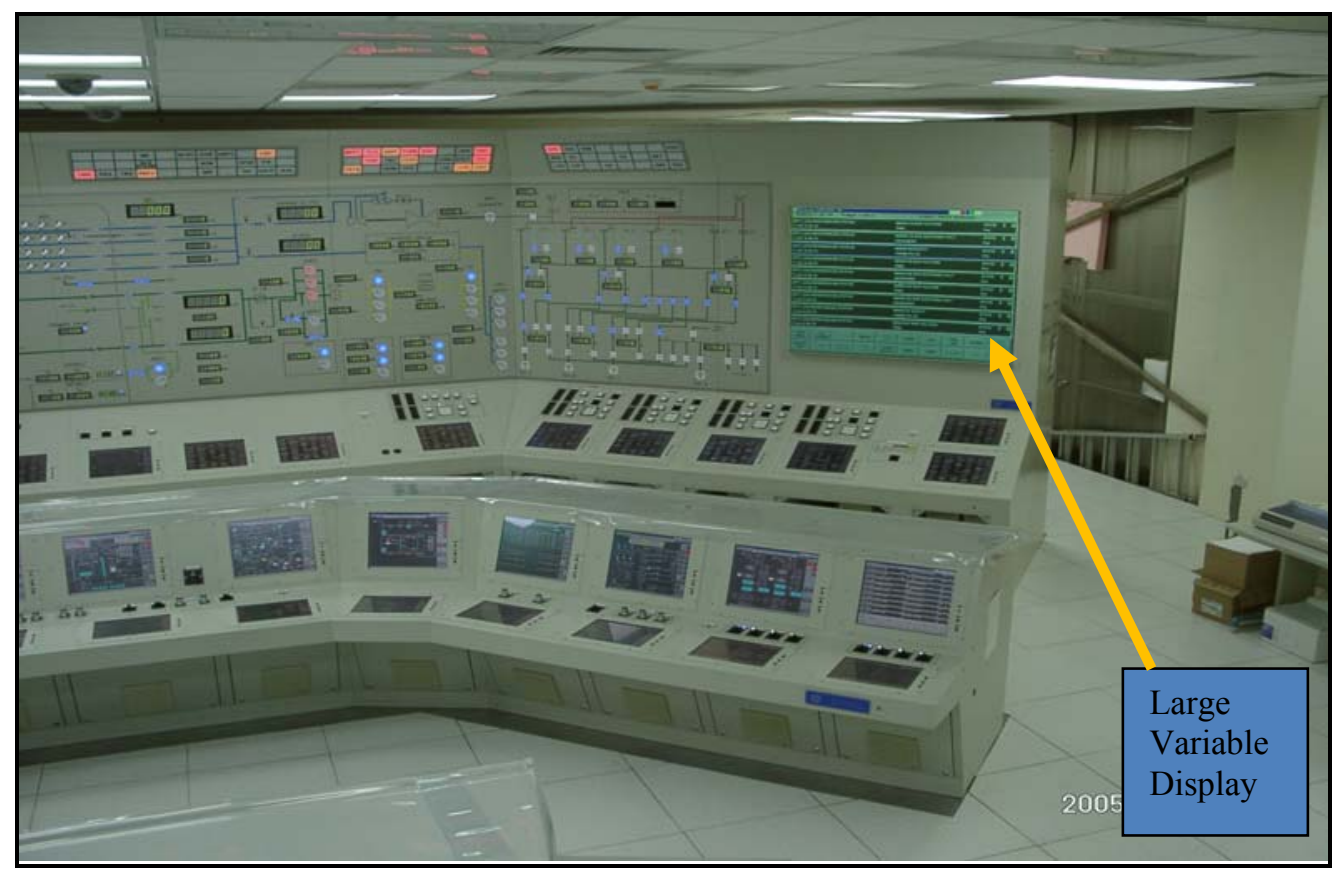

Figure 4.7. ABWR Control Room (Lungmen) Wide Display Panel Highlighting Large Variable Display 


\subsection{RI and OLE Training Needs and Priorities}

\subsection{What RIs and OLEs Need to Know: Task/KSA Inventory}

The final Task/KSA Inventory contains 54 specific items split 31 for RIs (including the 7 Ks that do not differ between positions and are counted only once in the overall inventory) and 30 for OLEs (also including the $7 \mathrm{Ks}$ ). The final list of tasks and knowledges required by RIs and OLEs is presented and discussed below, organized into the four taxonomy categories: knowledges $(\mathrm{K})$; operations and indications (Ops); management (Mgmt); and license-related (Lic). The first seven items represent broad categories of required knowledges, which are essentially the same for the two jobs. The remaining tasks are separated by job because RIs evaluate and report on operator performance in the actual operation of the plant, whereas OLEs evaluate and report on operator performance on examinations conducted on simulators, and then recommend licensing actions for the examinees. There are 16 pairs of operations tasks, plus one extra subtask for the RIs, resulting in a total of 33. There are 5 pairs of operations management tasks, for a total of 10 . There are 2 pairs of license-related tasks, for a total of 4 . Summing, then, there are 47 individual, job-specific tasks, plus 7 knowledges in the inventory, for a total of 54 items.

\subsubsection{Knowledges}

(K-1) knowledge of system function, design, and operation

(K-2) knowledge of the components in the system and the effects a malfunction of system component(s) would have on system performance, operation of the system, and the consequences (risk) associated with the malfunction

(K-3) knowledge of the physical connections and cause-effect relations between the system and other systems, including knowledge of the effect that loss or malfunction of the system would have on other systems

(K-4) knowledge of plant procedures, including administrative, normal, abnormal, and emergency procedures, and the site Emergency Plan

(K-5) knowledge of technical specifications and other regulatory requirements for operation and maintenance of the plant

(K-6) knowledge of the physical, chemical, thermal, and electrical properties and processes of the plant and their impact on plant behavior

(K-7) knowledge of reactivity effects associated with various plant evolutions and the impact the effects have on plant operations.

\subsubsection{Control Room Operations and Indications Tasks (Ops)}

(Ops-1) (RI) Inspect, monitor, assess, and report on operator performance in the use of CR indications to monitor and evaluate plant status and to identify trends. 
(OLE) Administer, document, and justify a licensing evaluation of operator performance on the use of CR indications to monitor and evaluate plant status and to identify trends.

(Ops-2) (RI) Inspect, monitor, assess, and report on operator performance in the use of procedures to operate and monitor plant systems and equipment.

(OLE) Administer, document, and justify a licensing evaluation of operator performance on the use of procedures to operate and monitor plant systems and equipment.

(Ops-3) (RI) Inspect, monitor, assess, and report on operator performance in reporting and responding to automatic plant actuations.

(OLE) Administer, document, and justify a licensing evaluation of operator performance in reporting and responding to automatic plant actuations.

(Ops-4) (RI) Determine/verify operability, functionality, and availability of plant systems and equipment and report findings to NRC.

(RI) Inspect, monitor, assess, and report on operator performance in the determination/ verification of operability, functionality, and availability of plant systems and equipment.

(OLE) Administer, document, and justify a licensing evaluation of operator performance in the determination/verification of operability, functionality, and availability of plant systems and equipment.

(Ops-5) (RI) Inspect, monitor, assess, and report on operator performance in monitoring and crosschecking diverse indications to verify and/or correctly understand plant conditions and to identify potential instrument or equipment malfunctions.

(OLE) Administer, document, and justify a licensing evaluation of operator performance in monitoring and cross-checking diverse indications to verify and/or correctly understand plant conditions and to identify potential instrument or equipment malfunctions.

(Ops-6) (RI) Inspect, monitor, assess, and report on operator performance in the use of plant procedures to respond to instrument or equipment malfunctions.

(OLE) Administer, document, and justify a licensing evaluation of operator performance in the use of plant procedures to respond to instrument or equipment malfunctions.

(Ops-7) (RI) Inspect, monitor, assess, and report on operator performance in obtaining, interpreting, and properly using normal operating procedures.

(OLE) Administer, document, and justify a licensing evaluation of operator performance in obtaining, interpreting, and properly using normal operating procedures.

(Ops-8) (RI) Inspect, monitor, assess, and report on operator performance in obtaining, interpreting, and properly using abnormal operating procedures. 
(OLE) Administer, document, and justify a licensing evaluation of operator performance in obtaining, interpreting, and properly using abnormal operating procedures.

(Ops-9) (RI) Inspect, monitor, assess, and report on operator performance in obtaining, interpreting, and properly using emergency operating procedures.

(OLE) Administer, document, and justify a licensing evaluation of operator performance in obtaining, interpreting, and properly using emergency operating procedures.

(Ops-10) (RI) Inspect, monitor, assess, and report on operator performance in obtaining, interpreting, and properly implementing the emergency classification guidelines and Emergency Plan.

(OLE) Administer, document, and justify a licensing evaluation of operator performance in obtaining, interpreting, and properly implementing the emergency classification guidelines and Emergency Plan.

(Ops-11) (RI) Inspect, monitor, assess, and report on operator performance in obtaining, interpreting, and properly using $\mathrm{CR}$ reference materials such as drawings, charts, plots and other operator aides.

(OLE) Administer, document, and justify a licensing evaluation of operator performance in obtaining, interpreting, and properly using CR reference materials such as drawings, charts, plots and other operator aides.

(Ops-12) (RI) Inspect, monitor, assess, and report on operator performance in the use of plant guidelines and procedures to respond to alarms.

(OLE) Administer, document, and justify a licensing evaluation of operator performance in the use of plant guidelines and procedures to respond to alarms.

(Ops-13) (RI) Inspect, monitor, assess, and report on operator performance in the use of procedures for monitoring and operating systems for plant radiation control and release.

(OLE) Administer, document, and justify a licensing evaluation of operator performance in the use of procedures for monitoring and operating systems for plant radiation control and release.

(Ops-14) (RI) Inspect, monitor, assess, and report on operator performance in the use of the plant computer to display additional plant status, alarms, and trend information beyond that available on panel instrumentation.

(OLE) Administer, document, and justify a licensing evaluation of operator performance in the use of the plant computer to display additional plant status, alarms, and trend information beyond that available on panel instrumentation.

(Ops-15) (RI) Inspect, monitor, assess, and report on operator performance in using administrative procedures to provide shift turnover information. 
(OLE) Administer, document, and justify a licensing evaluation of operator performance in using administrative procedures to provide shift turnover information.

(Ops-16) (RI) Gather information and communicate with the NRC during declared emergencies.

(OLE) Gather information and communicate with the NRC during declared emergencies.

\subsubsection{Operations Management Tasks (Mgmt)}

(Mgmt-1) (RI) Inspect, monitor, assess, and report on CR command and control performance.

(OLE) Administer, document, and justify a licensing evaluation of CR command and control performance.

(Mgmt-2) (RI) Inspect, monitor, assess, and report on CR performance in the use of plant procedures, $\mathrm{CR}$ indications, and the communications system to monitor and control tasks performed outside the CR.

(OLE) Administer, document, and justify a licensing evaluation of CR performance in the use of plant procedures, CR indications, and the communications system to monitor and control tasks performed outside the CR.

(Mgmt-3) (RI) Inspect, monitor, assess, and report on operator performance in verbally communicating with the crew, plant staff, supervisory personnel, and management.

(OLE) Administer, document, and justify a licensing evaluation of operator performance in verbally communicating with the crew, plant staff, supervisory personnel, and management.

(Mgmt-4) (RI) Inspect, monitor, assess, and report on operator performance in completing and archiving logs, records, or other required documentation.

(OLE) Administer, document, and justify a licensing evaluation of operator performance in completing and archiving logs, records, or other required documentation.

(Mgmt-5) (RI) Inspect, monitor, assess, and report on operator performance in the use of administrative procedures associated with risk-assessment activities.

(OLE) Administer, document, and justify a licensing evaluation of operator performance in the use of administrative procedures associated with risk assessment activities.

\subsubsection{Plant License-Related Tasks (Lic)}

(Lic-1) (RI) Inspect, monitor, assess, and report on the Licensee's compliance with NRC regulatory requirements for operator performance in recognizing, documenting, tracking, and resolving Technical Specifications and other reportable conditions. 
(OLE) Administer, document, and justify a licensing evaluation of operator performance in recognizing, documenting, tracking, and resolving technical specifications and other reportable conditions.

(Lic-2) (RI) Inspect, monitor, assess, and report on the licensee's compliance with NRC regulatory requirements for operator performance in determining and implementing the desired operational path forward based on plant status information and administrative procedures and directives.

(OLE) Administer, document, and justify a licensing evaluation of operator performance in determining and implementing the desired operational path forward based on plant status information and administrative procedures and directives.

\subsection{Results of the DIF Analysis to Identify Priorities for Training RIs and OLES}

The team performed an analysis of the Task/KSA Inventory items to rate the difficulty of performance, importance to safety, and frequency of performance of each of the 61 selected KSA items, as described in Section 3.2.2. The DIF analysis focuses on difficulty, importance to safety, and frequency in terms of performance of the task, not training. A set of the knowledges (K-1, K-2, K-3, K-6 and K-7) were rated in a different way, as concepts required in order to successfully perform the tasks in the inventory. The complete set of DIF ratings is presented in Chapter 8, Table 8.2. Table 5.1 shows the Task/KSA Inventory items receiving the highest and lowest DIF (i.e., combined) scores in this analysis. Based on these ratings, these items are considered to have the highest and lowest training priority.

Table 5.1. Task/KSA Inventory Items Receiving the Highest and Lowest DIF Scores

\begin{tabular}{|c|l|c|l|c|l|c|c|l|c|}
\hline Rank & $\begin{array}{c}\text { Top 10 } \\
\text { RI }\end{array}$ & $\begin{array}{c}\text { DIF } \\
\text { Score }\end{array}$ & $\begin{array}{c}\text { Top 10 } \\
\text { OLE }\end{array}$ & $\begin{array}{c}\text { DIF } \\
\text { Score }\end{array}$ & $\begin{array}{c}\text { Top 10 } \\
\text { All }\end{array}$ & $\begin{array}{c}\text { DIF } \\
\text { Score }\end{array}$ & Rank & $\begin{array}{c}\text { Lowest 10 } \\
\text { All }\end{array}$ & $\begin{array}{c}\text { DIF } \\
\text { Score }\end{array}$ \\
\hline 1 & Ops-16 & 10 & K-2 & 8 & Ops-16r & 10 & 61 & Mgmt-4o & 1 \\
\hline 2 & Ops-13 & 8 & K-3 & 8 & Ops-13r & 8 & 60 & Ops-15r & 2 \\
\hline 3 & Ops-10 & 8 & K-6 & 8 & K-2o & 8 & 59 & Mgmt-3o & 2 \\
\hline 4 & Ops-3 & 8 & K-7 & 8 & K-3o & 8 & 58 & Ops-14r & 3 \\
\hline 5 & Ops-9 & 8 & K-1 & 7 & K-6o & 8 & 57 & Ops-7o & 4 \\
\hline 6 & K-2 & 7 & K-4 & 7 & K-7o & 8 & 56 & Ops-2o & 4 \\
\hline 7 & K-4 & 7 & Ops-5 & 7 & Ops-10r & 8 & 55 & Ops-15o & 4 \\
\hline 8 & K-6 & 7 & Ops-9 & 7 & Ops-3r & 8 & 54 & Ops-14o & 4 \\
\hline 9 & K-7 & 7 & K-5 & 6 & Ops-9r & 8 & 53 & Ops-12o & 4 \\
\hline 10 & Lic-2 & 7 & Mgmt-1 & 6 & K-1o & 7 & 52 & Ops-8o & 5 \\
\hline $\begin{array}{l}\text { r }=\text { RI. } \\
\text { o }=\text { OLE. }\end{array}$
\end{tabular}

For RIs, five of the top ten priority items are (Ops), which are rated as a higher priority than the four (K) and one (Lic) item that compose the rest of the top priority items. For OLEs, the six highest priority 
items are (K), followed by two (Ops), one more (K), and one (Mgmt) item. Only one item, Ops-16 for RIs (gather information and communicate with the NRC during declared emergencies), received the highest priority rating among the items, with a DIF score of "10." The DIF scores varied widely across the Task/KSA Inventory items and two positions. Other conclusions from this analysis of the Task/KSA Inventory items include the following:

- The 15 items with the highest DIF scores are either knowledge or operations topics.

- The highest rated Licensing item was 16 th in rank.

- Roughly half of the 20 items receiving a DIF score of 7 or more concerned System Knowledge.

Table 5.2 shows the items receiving the highest and lowest difficulty and importance scores. For RIs, no items were rated " 5 " in difficulty to perform. Seventeen items were rated "4," eight of which were Ops items. For OLEs, the items rated most difficult to perform were all knowledge items - those dealing with the overall plant - which were each rated "5." The items rated most important to safety for RIs were all Ops items; for OLEs, 11 items were rated as most important for safety (score " 5 "), 5 of which were $(\mathrm{K}), 5$ were (Ops), and 1 was (Mgmt). The complete scoring tabulations are shown in Appendix B.

Table 5.2. Highest and Lowest Scores for Difficulty and Importance

\begin{tabular}{|c|c|c|c|c|c|c|c|}
\hline \multicolumn{4}{|c|}{ Highest Difficulty to Perform } & \multicolumn{4}{|c|}{ Highest Importance to Safety } \\
\hline RI & Score & OLE & Score & RI & Score & OLE & Score \\
\hline 17 items & 4 & $\mathrm{~K}-1$ & 5 & Ops-3 & 5 & 11 items & 5 \\
\hline $8 \mathrm{Ops}$ & & $\mathrm{K}-2$ & 5 & Ops-4 & 5 & $5 \mathrm{~K}$ & \\
\hline $6 \mathrm{~K}$ & & K-3 & 5 & Ops-5 & 5 & 5 Ops & \\
\hline $2 \mathrm{Lic}$ & & K-6 & 5 & Ops-9 & 5 & $1 \mathrm{Mgmt}$ & \\
\hline $1 \mathrm{Mgmt}$ & & $\mathrm{K}-7$ & 5 & Ops-16 & 5 & & \\
\hline \multicolumn{4}{|c|}{$\begin{array}{l}\text { Lowest Difficulty to Perform } \\
\end{array}$} & \multicolumn{4}{|c|}{ Lowest Importance to Safety } \\
\hline Ops-14 & 2 & Ops-15 & 1 & Ops-15 & 2 & Mgmt-3 & 2 \\
\hline Ops-15 & 2 & Ops-16 & 1 & 6 items & 3 & Mgmt-4 & 2 \\
\hline 11 items & 3 & Mgmt-3 & 1 & $5 \mathrm{Mgmt}$ & & 4 items & 3 \\
\hline & & Mgmt-4 & 1 & $1 \mathrm{Ops}$ & & $4 \mathrm{Ops}$ & \\
\hline & & 6 items & 2 & & & & \\
\hline
\end{tabular}

Table 5.3 shows the items receiving the highest and lowest frequency scores. Note that low frequency of performance creates a higher priority for training. RIs reported more items being performed with low frequency (Ops-16 at the lowest; three [Ops] and one [Mgmt] task at the next lowest frequency) than OLEs, who reported no tasks being performed at the lowest frequency. OLEs reported performing 18 tasks at the highest frequency, of which 11 were (Ops) and 6 were (K). 
Table 5.3. Items Receiving the Highest and Lowest Scores for Frequency

\begin{tabular}{|l|c|c|c|c|c|c|c|}
\hline \multicolumn{4}{|c|}{ Lowest Frequency of Performance } & \multicolumn{4}{c|}{ Highest Frequency of Performance } \\
\hline \multicolumn{1}{|c|}{ RI } & Score & OLE & Score & RI & Score & OLE & Score \\
\hline Ops-16 & 1 & Mgmt-4 & 2 & Ops-1 & 5 & 18 items & 5 \\
\hline Ops-13 & 2 & 6 items & 3 & 12 items & 4 & 11 Ops & \\
\hline Ops-10 & 2 & 4 Ops & & 7 Ops & & $6 \mathrm{~K}$ & \\
\hline Ops-9 & 2 & $2 \mathrm{Mgmt}$ & & $3 \mathrm{~K}$ & & $1 \mathrm{Mgmt}$ & \\
\hline Mgmt-2 & 2 & & & $\begin{array}{l}1 \mathrm{Lic} ; \\
1 \mathrm{Mgmt}\end{array}$ & & & \\
\hline
\end{tabular}

\subsection{Difficulty of Learning and Importance of Using a Simulator to Learn Task/KSA Inventory Items}

The expert panels conducting the DIF analysis also rated the Task/KSA Inventory items with respect to the difficulty of learning them. They also rated each item with respect to the importance of using a simulator to learn the item. Table 5.4 presents the highest rated Task/KSA Inventory items with respect to these two dimensions.

Table 5.4. Task/KSA Inventory Items Most Difficult to Learn and for Which Simulator Use is Most Important for Learning

\begin{tabular}{|c|c|l|c|c|c|c|c|}
\hline \multicolumn{4}{|c|}{ Most Difficult to Learn } & \multicolumn{3}{c|}{ Simulator Most Important to Learning } \\
\hline RI & Score & OLE & Score & RI & Score & OLE & Score \\
\hline 18 items & 4 & K-2 & 5 & Ops-10 & 5 & Ops-9 & 5 \\
\hline 11 Ops & & K-3 & 5 & Ops-3 & 5 & Mgmt-1 & 5 \\
\hline 6 K & & K-6 & 5 & Ops-9 & 5 & Ops-1 & 5 \\
\hline 1 Lic & & K-7 & 5 & 12 items & 4 & Ops-6 & 5 \\
\hline & & K-1 & 5 & 9 Ops & & Ops-3 & 5 \\
\hline & & & & 2 K & & Ops-8 & 5 \\
\hline & & & & 1 Mgmt & & Ops-12 & 5 \\
\hline & & & & & & Ops-7 & 5 \\
\hline
\end{tabular}

None of the RI's Task/KSA Inventory items were rated "very difficult" to learn. For OLEs, the knowledges of plant design details and interactions between systems and components, including malfunctions of components were rated "very difficult" to learn. This may represent the immediacy of the OLE role in understanding the integrated operations of the plant and designing simulations to appropriately exercise and evaluate the performance of license candidates in real time.

Simulators were rated as important for learning the Task/KSA Inventory items involving emergencies and automatic plant actuations (e.g., reactor trip). These items require conceptual integration of large amounts of system and component operation and interaction information in real time to understand and keep abreast of the evolving plant status. In addition, the raters also assigned this highest rating to several 
other OLE tasks involving responses to malfunctions, including use of normal and abnormal operating procedures and the assessment of CR command and control.

Analysis of these ratings yielded the following conclusions:

- The importance of using a simulator to train RIs and OLEs for competent job performance is high for nearly half the Task/KSA Inventory items and low for nearly one third of the items. The importance of using a simulator addresses the desirability of using a simulator for training, but it does not address the need for any specific level of fidelity.

- The importance of using a simulator for learning varies little across the two positions.

- There is a moderate degree of correspondence between training priority and importance of using a simulator to train (e.g., experts say a simulator is of high importance for learning on roughly half the 20 items that have a high priority for training).

- The average difficulty rating of learning for the 20 items rated "high priority" is 4.2 (out of 5).

- The average importance rating of using a simulator for learning the 20 items rated "high priority" is 3.8 (out of 5).

\subsection{Types and Levels of KSAs of the Task/KSA Inventory Items}

The types and levels of knowledge required for RIs and OLEs to perform each of the tasks in the task inventory were rated by consensus discussions of a panel of subject matter experts, as described in Section 3.2, where the types and levels of KSAs and the coding scale are described. The framework and coding scale are also presented in Appendix C.

Because of the broad scope of each of the tasks in the inventory, and the similarities of the RI and OLE jobs, the panel concluded that it was not possible to differentiate between the two jobs in terms of the KSA levels needed. Thus, the expert panel assigned the same ratings to RI and OLE needs for factual and procedural knowledges, concepts, and psychomotor skills. Similarly, the panel was unable to differentiate between the two jobs with respect to the need for internal facts (from memory) and external facts (from reference materials).

For operations tasks (Ops-1 through Ops-16) the need for factual knowledge was rated in all cases as "essential to recall basic facts from memory." For 10 of the 16 tasks a higher rating was given, specifying the need to recall facts "quickly." Most of the tasks requiring quick application of factual knowledge had to do with operator actions when responding to equipment malfunctions or emergencies. Details of this differentiation are presented in Table 5.5.

For management tasks (Mgmt-1 through Mgmt-5), the need for factual knowledge was rated as "essential to recall basic facts from memory" for the tasks assessing the control of tasks performed outside the CR and for risk assessment activities. The other three management tasks, addressing CR command and control, communications, and completing logs and records, received the lesser rating of "essential to recognize that observed information accurately represents something known to be true." 
Table 5.5. Ratings of Task/KSAs on Levels of Knowledges and Skills Needed

\begin{tabular}{|c|c|c|c|c|c|}
\hline \multirow[b]{2}{*}{ Type } & \multirow{2}{*}{\begin{tabular}{|c|}
$\begin{array}{c}\text { Level Required for Successful } \\
\text { Performance of Task }\end{array}$ \\
\end{tabular}} & \multicolumn{4}{|c|}{ Task/KSA Inventory Items } \\
\hline & & Knowledges & Ops & Mgt & Lic \\
\hline \multicolumn{6}{|c|}{ FACTS-Internal knowledge of facts } \\
\hline 3 & $\begin{array}{l}\text { Essential to recognize that } \\
\text { observed information } \\
\text { accurately represents } \\
\text { something known to be true. }\end{array}$ & & & $\begin{array}{l}\text { Mgmt-1; } \\
\text { Mgmt-3; } \\
\text { Mgmt-4 }\end{array}$ & \\
\hline 4 & $\begin{array}{l}\text { Essential to recall basic facts from } \\
\text { memory. }\end{array}$ & $\mathrm{K}-4 ; \mathrm{K}-5$ & $\begin{array}{l}\text { Ops-1; Ops-4b; Ops-7; Ops-10; } \\
\text { Ops-11; Ops-14; Ops-15 }\end{array}$ & $\begin{array}{l}\text { Mgmt-2; } \\
\text { Mgmt-5 }\end{array}$ & $\begin{array}{l}\text { Lic-1; } \\
\text { Lic-2 }\end{array}$ \\
\hline 5 & $\begin{array}{l}\text { Essential to recall or recognize } \\
\text { basic facts from memory } \\
\text { quickly. }\end{array}$ & & $\begin{array}{l}\text { Ops-2; Ops-3; Ops-4a; Ops-5; } \\
\text { Ops-6; Ops-8; Ops-9; Ops-12; } \\
\text { Ops-13; Ops-16 }\end{array}$ & & \\
\hline \multicolumn{6}{|c|}{ FACTS-External knowledge of facts } \\
\hline 3 & $\begin{array}{l}\text { Essential to use external } \\
\text { information to verify that } \\
\text { observed information } \\
\text { accurately represents } \\
\text { something known to be true. }\end{array}$ & & & $\begin{array}{l}\text { Mgmt-1; } \\
\text { Mgmt-3; } \\
\text { Mgmt-4 }\end{array}$ & \\
\hline 4 & $\begin{array}{c}\text { Essential to use external resources } \\
\text { to access factual information. }\end{array}$ & $\mathrm{K}-4 ; \mathrm{K}-5$ & $\begin{array}{l}\text { Ops-1; Ops-4b; Ops-7; Ops-10; } \\
\text { Ops-11; Ops-14; Ops-15 }\end{array}$ & $\begin{array}{l}\text { Mgmt-2; } \\
\text { Mgmt-5 }\end{array}$ & $\begin{array}{l}\text { Lic-1; } \\
\text { Lic-2 }\end{array}$ \\
\hline 5 & $\begin{array}{l}\text { Essential to use external resources } \\
\text { to quickly access factual } \\
\text { information or verify observed } \\
\text { information. }\end{array}$ & & $\begin{array}{l}\text { Ops-2; Ops-3; Ops-4a; Ops-5; } \\
\text { Ops-6; Ops-8; Ops-9; Ops-12; } \\
\text { Ops-13; Ops-16 }\end{array}$ & & \\
\hline \multicolumn{6}{|c|}{ K-1; K-2; K-3; K-6; and K-7 (Plant Knowledge) [From the Task/KSA Inventory] } \\
\hline 1 & None (No knowledge needed) & & & & \\
\hline 2 & Understand & $\mathrm{K}-4 ; \mathrm{K}-5$ & All Ops-items & $\begin{array}{l}\text { Mgmt-1; } \\
\text { Mgmt-2; } \\
\text { Mgmt-5 }\end{array}$ & $\begin{array}{l}\text { Lic-1; } \\
\text { Lic-2 }\end{array}$ \\
\hline \multicolumn{6}{|c|}{ PROCEDURAL Knowledge } \\
\hline 2 & $\begin{array}{l}\text { Only need to know the purpose of } \\
\text { the thing being done }\end{array}$ & & & Mgmt-1 & \\
\hline 3 & $\begin{array}{l}\text { Need to know how it is done, but } \\
\text { not how to execute it. }\end{array}$ & $\mathrm{K}-4 ; \mathrm{K}-5$ & $\begin{array}{l}\text { Ops-1; Ops-2; Ops-3; Ops-4a; } \\
\text { Ops-4b; Ops-5; Ops-6; Ops-8; } \\
\text { Ops-11; Ops-12; Ops-13; } \\
\text { Ops-14; Ops-15 }\end{array}$ & $\begin{array}{l}\text { Mgmt-2; } \\
\text { Mgmt-3; } \\
\text { Mgmt-4 }\end{array}$ & $\begin{array}{l}\text { Lic-1; } \\
\text { Lic-2 }\end{array}$ \\
\hline 4 & $\begin{array}{l}\text { Need to know how to execute it, } \\
\text { but effortful, awkward, or } \\
\text { slow. }\end{array}$ & & Ops-7; Ops-9; Ops-10; Ops-16 & Mgmt-5 & \\
\hline \multicolumn{6}{|c|}{ PSYCHOMOTOR Skills Motor } \\
\hline 1 & Not needed & $\mathrm{K}-4 ; \mathrm{K}-5$ & All & All & $\begin{array}{l}\text { Lic-1; } \\
\text { Lic-2 }\end{array}$ \\
\hline 2 & Familiarity Needed & & & & \\
\hline \multicolumn{6}{|c|}{ PSYCHOMOTOR Skills Visual } \\
\hline 1 & Not needed & $\mathrm{K}-4 ; \mathrm{K}-5$ & & Mgmt-3 & \\
\hline 2 & Familiarity Needed & & All & $\begin{array}{l}\text { Mgmt-1; } \\
\text { Mgmt-2; } \\
\text { Mgmt-4; } \\
\text { Mgmt-5 }\end{array}$ & $\begin{array}{l}\text { Lic-1; } \\
\text { Lic-2 }\end{array}$ \\
\hline
\end{tabular}


Table 5.5. (contd)

\begin{tabular}{|c|c|c|c|c|c|}
\hline \multirow[b]{2}{*}{ Type } & \multirow{2}{*}{$\begin{array}{c}\text { Level Required for Successful } \\
\text { Performance of Task }\end{array}$} & \multicolumn{4}{|c|}{ Task/KSA Inventory Items } \\
\hline & & Knowledges & Ops & Mgt & Lic \\
\hline \multicolumn{6}{|c|}{ PSYCHOMOTOR Skills Auditory } \\
\hline 1 & Not needed & K-4; K-5 & & $\begin{array}{l}\text { Mgmt-4; } \\
\text { Mgmt-5 }\end{array}$ & \\
\hline 2 & Familiarity Needed & & All & $\begin{array}{l}\text { Mgmt-1; } \\
\text { Mgmt-2; } \\
\text { Mgmt-3 }\end{array}$ & $\begin{array}{l}\text { Lic-1; } \\
\text { Lic-2 }\end{array}$ \\
\hline \multicolumn{6}{|c|}{ PSYCHOMOTOR Skills Tactile } \\
\hline 1 & Not needed & $\mathrm{K}-4 ; \mathrm{K}-5$ & All & All & $\begin{array}{l}\text { Lic-1; } \\
\text { Lic-2 }\end{array}$ \\
\hline 2 & Familiarity Needed & & & & \\
\hline
\end{tabular}

The need for factual knowledge in both licensing tasks (Lic-1 and Lic-2) received the rating "essential to recall basic facts from memory."

The two knowledge items that involved knowledges applied inside the CR, including knowledge of procedures (K-4, all procedure types including EOPs) and knowledge of regulatory requirements (K-5, technical specifications and all others), received the rating "essential to recall basic facts from memory." The remaining knowledges (K-1, K-2, K-3, K-6, and K-7) were rated differently-as concepts required in order to successfully perform the tasks in the inventory. The panel rated the need for these concepts at the level of "understand" for all tasks. They felt that there was no need to further analyze or evaluate the facts or to create new concepts (the possible higher ratings that were not assigned).

The importance of procedures to plant operations is reflected in the importance of procedural knowledge, which was rated separately for each item in the inventory. The higher rating of "need to know how to execute but not fluently," was given for the operations tasks involving prompt actions, (including Ops-7, normal operations; Ops-9, EOPs; Ops-10, emergency plan; and Ops-16, communication with NRC during declared emergencies). The lesser rating of "need to know how it is done, but not how to execute it" was given to all other Ops tasks.

The ratings of procedural knowledges for the management tasks showed the greatest variation, spanning three of the six possible rating categories. The higher rating of "need to know how to execute it but not fluently" was given to Mgmt-5 (assessing the use of administrative procedures associated with risk assessment activities). The lesser rating of "need to know how it is done, but not how to execute it" was given to three other Mgmt activities (Mgmt-2, addressing the monitoring and control of tasks performed outside the CR; Mgmt-3, addressing verbal communication by operators; and Mgmt-4, addressing completion of logs, records and other required documentation). The even lower rating of "only need to know the purpose" was given to the procedural knowledge required for Mgmt-1 (evaluation of CR command and control).

For the two licensing tasks, the ratings of procedural knowledge were "need to know how it is done, but not how to execute it" for both (Lic-1, documenting, tracking and resolution of technical specifications and other reportable conditions; and Lic-2, determination of the desired operational path forward). 
For the two procedural knowledge tasks the ratings of procedural knowledge were "need to know how it is done, but not how to execute it" for both (K-4, knowledge of all plant procedures; and K-5, knowledge of technical specifications and other regulatory requirements).

The ratings of psychomotor skills required for all tasks were uniformly low - regulators do not perform plant operations. Motor skill and tactile skill were rated as "not needed" for all tasks. "Familiarity needed" was given for visual skill to all of the tasks except Mgmt-3, addressing verbal communications; the two licensing tasks (Lic-1 addressing reportable conditions; and Lic-2 addressing determination of the desired operational path forward), and the two knowledge tasks (K-4, addressing procedural knowledges; and K-5, addressing knowledge of technical specifications and other regulatory requirements). For these, visual skill was rated as "not needed." Similarly, "familiarity needed" was given for auditory skill to all items except Mgmt-4, addressing logs and records; and Mgmt-5, addressing risk-assessment activities, and the two knowledge tasks (K-4, addressing procedural knowledges, and $\mathrm{K}-5$, addressing knowledge of technical specifications and other regulatory requirements). For these, auditory skill requirement was rated as "not needed."

The expert panel that rated the Task/KSA Inventory items in terms of the type and level of knowledge, skill or ability required to perform the item competently determined that there was a high degree of similarity between the two positions and for items within each of the four categories of Task/KSAs.

The implication of the above KSA discussion for simulator fidelity is that the simulator needs to provide sufficient fidelity for OLE and RI trainees to learn to recognize correct actions performed by operators or operator candidates. The incumbents identified a few knowledge items that need to be taught at the "essential to recall from memory level, but most knowledge items only need to be learned at the "understand" level. Knowledge of plant procedures was identified as particularly important. Trainees must learn to execute the procedures, although not necessarily fluently. The trainees do not need to develop any specific psychomotor skills beyond auditory and visual familiarity, because they do not actually manipulate controls on the job.

\subsection{Role of Simulator/HSI Systems in Training the Task/KSA Inventory Items}

\subsubsection{Importance of HSI Systems to Task/KSA Inventory Items}

Using the rating process described in Section 3.2, the expert panel found a high degree of similarity in the importance of HSI systems to Task/KSAs for the two positions (RI and OLE), with a few exceptions.

Figures 5.1, 5.2, and 5.3 present the results of the expert panel ratings of the importance of each of the eight CR HSI systems to the performance of each Task/KSA Inventory item. Figures 5.1 and 5.2 present the same information in different formats, organized by the overall importance of HSI systems to the task. Specifically, Figure 5.1 shows the percentage of Task/KSA Inventory items for which each HSI system is of high, medium, or low importance, while Figure 5.2 shows this information in terms of the number of Task/KSA Inventory items. Figure 5.3 shows the pattern of importance ratings for the eight HSI systems by inventory item. 


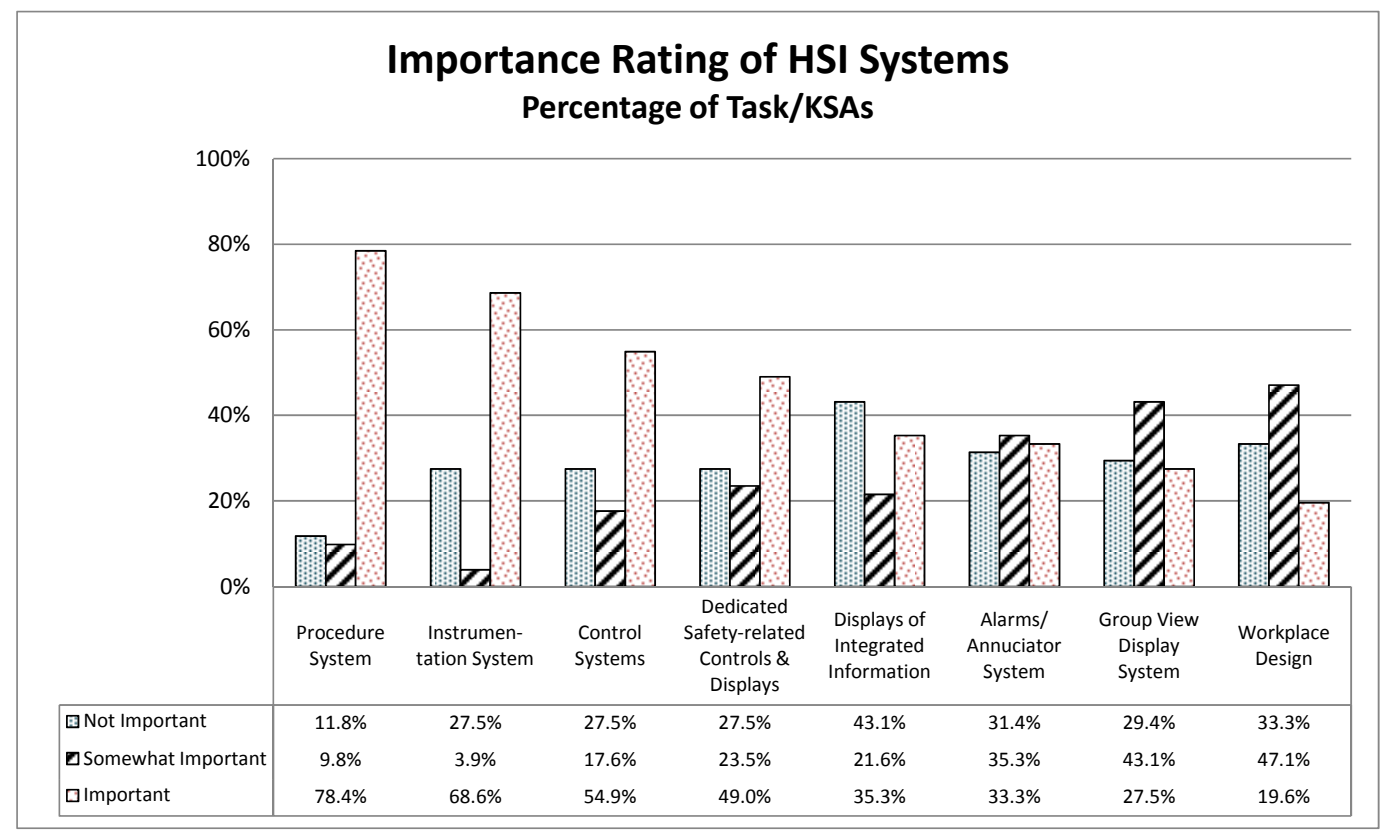

Figure 5.1. Percentage of Tasks/KSAs Inventory Items for Which Each HSI Systems is Important

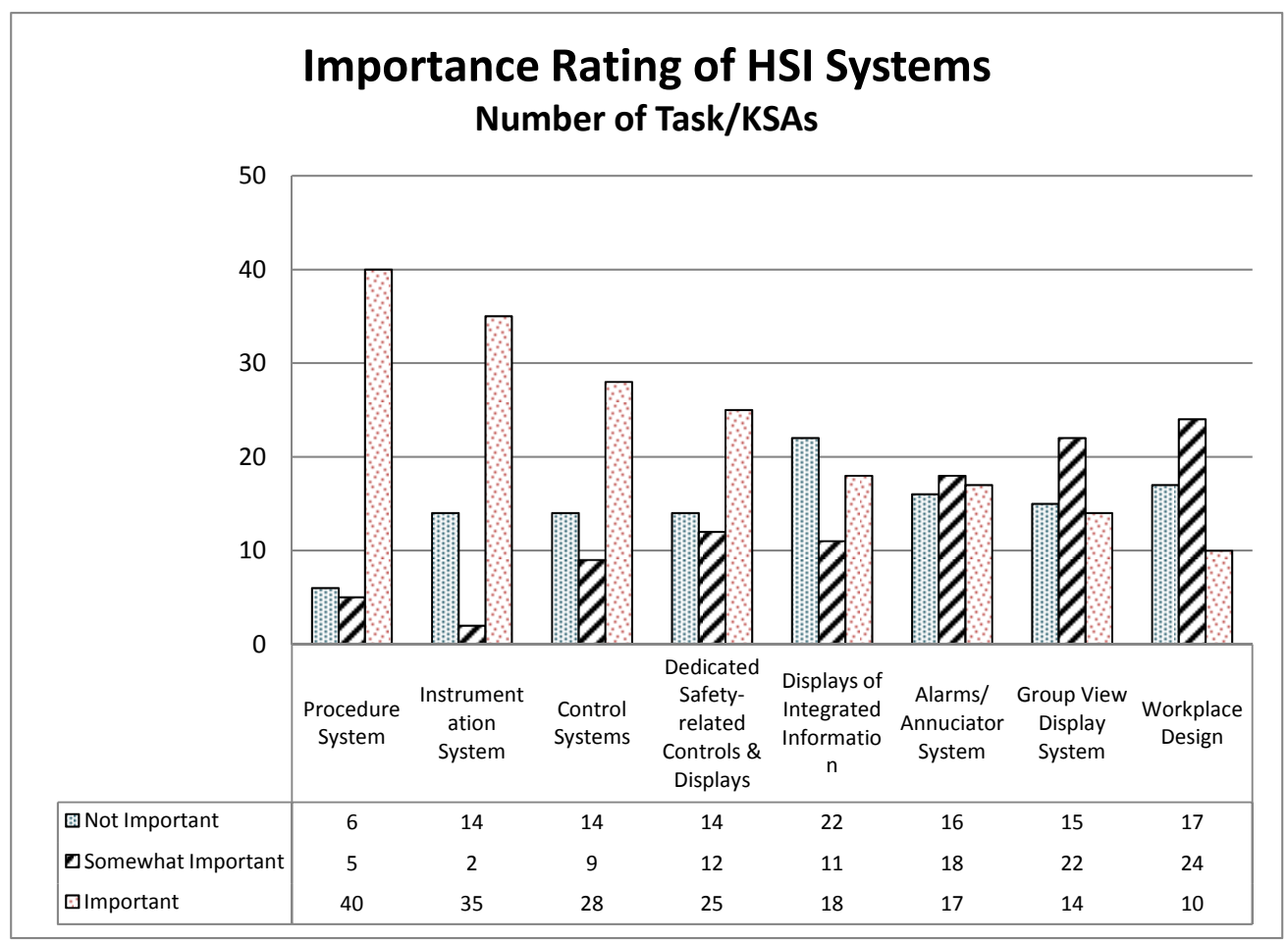

Figure 5.2. Number of Tasks/KSAs Inventory Items for Which Each HSI System is Important 


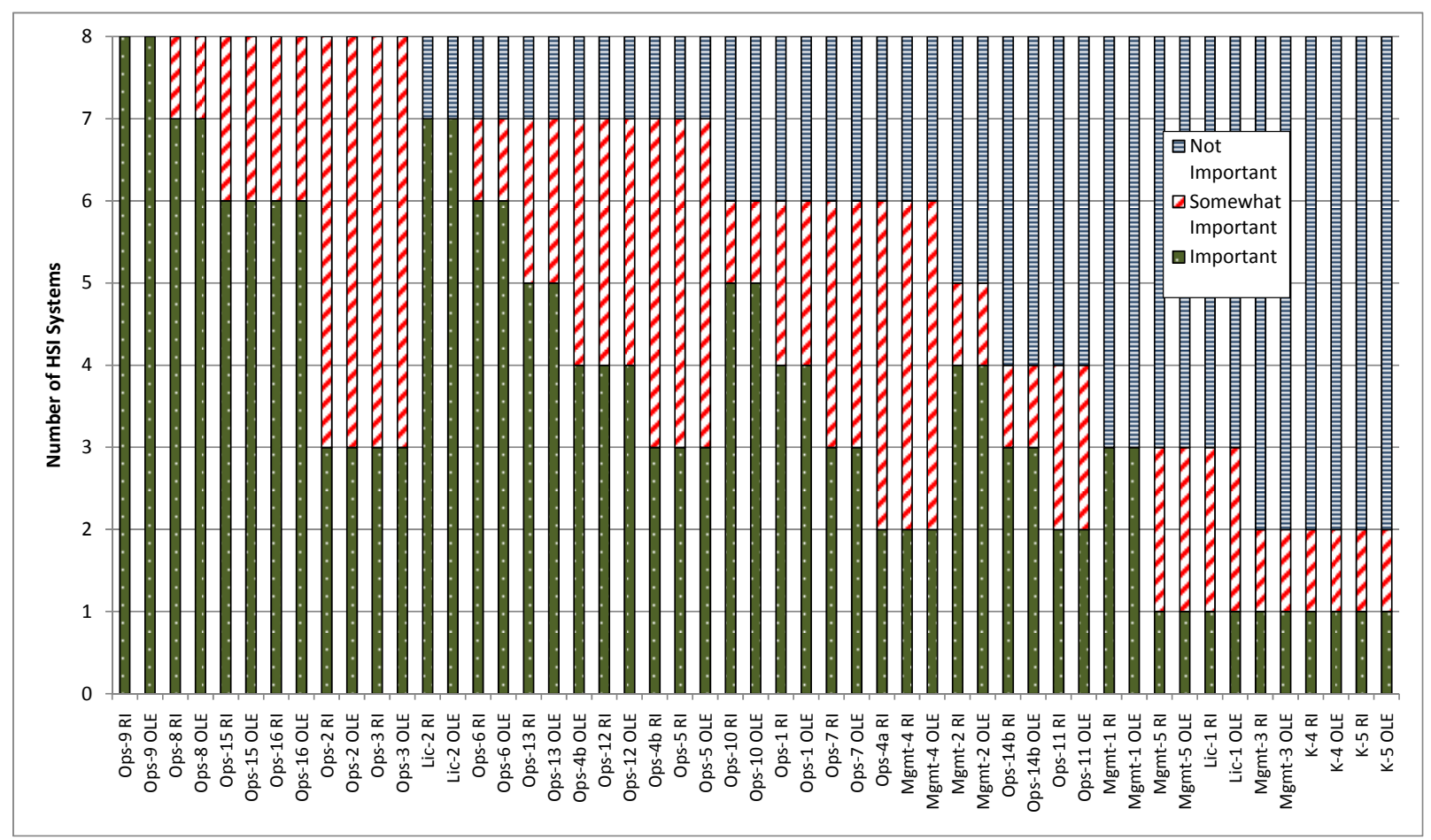

Figure 5.3. Number of CR HSI Systems Rated of High, Medium, and Low Importance to Tasks/KSA Inventory Items

From Figures 5.1 and 5.2, it is immediately apparent that the procedure system is highly important to the greatest number of tasks in the inventory (78\%), followed by the instrumentation system (69\%), the control systems (55\%), and the dedicated safety-related controls and displays (49\%). Overall, these three figures illustrate that each of the systems is important to some Task/KSA Inventory items, and that all inventory items rely on a number of HSI systems. No HSI system was rated as important to fewer than 10 inventory items.

As seen in Figure 5.3, all eight HSI systems are highly important to performance of Ops-9 (addressing operator performance in obtaining, interpreting and properly using emergency operating procedures). This is true for both RIs and OLEs. HSI systems are generally of high importance for most of the Ops tasks. Lic-2 (addressing operator performance in determining and implementing the desired operational path forward based on plant status information and administrative procedures and directives) is the only non-Ops task that has seven HSI systems important to task performance. The ratings for RIs and OLEs are very similar for all Tasks/KSAs except Ops-4b (addressing operator performance in the determination/verification of operability, functionality, and availability of plant systems and equipment).

\subsection{Implications for Physical Fidelity of TTC Simulators in Advanced Reactor Training}

The Task/KSA Inventory for RI and OLE positions, which was developed and validated with current NRC RIs and OLEs, provides a basis for examining training priorities and the role of simulator training in preparing staff to regulate and oversee current and new reactors. Although developed for the RI and OLE 
positions relative to current generation reactors, the inventory is also expected to apply to these positions for Gen III and III+ reactors. The majority of RI inventory items involve inspecting, monitoring, assessing, and reporting on operator performance, while the majority of OLE inventory items involve administering, documenting, and justifying a licensing evaluation of an operator candidate's performance on the same operator tasks. The high priority training items identified through the DIF analysis differed by position, with five operations (Ops) items among the top ten for RIs and six knowledges (K) items among the top ten for OLEs.

All eight HSI systems are important to the RI and OLE jobs, particularly for the operations (Ops) tasks and for one of the licensing (Lic) tasks. Procedure systems were rated important for the greatest number of Task/KSA Inventory items (78\%); workplace design was rated important for the fewest (20\%). This indicates that special attention to the physical fidelity of procedures of training simulators may be warranted.

Simulator training is important for nearly half of the Task/KSA Inventory items, and the items for which it is important are similar for RI and OLE positions. Half of the items rated high in importance of using a simulator for learning were among the items receiving the highest rating in terms of training priority. The top 20 training priority items, overall, averaged 4.2 (out of 5) in terms of importance of using a simulator for learning and 3.8 out of 5 in terms of difficulty of learning.

The analysis of type and level of KSA required for each of the Task/KSA Inventory items concluded that the RI and OLE positions did not require psychomotor motor or tactile skills and required auditory and visual skills only at the level of familiarity. The analysis identified considerable variability in the level of procedural knowledge needed by RIs and OLEs, ranging from "need to know how to execute it, but not fluently" to "only need to know the purpose of the thing being done." This indicates that the training simulator needs to provide physical fidelity that is sufficient for students to learn to recognize correct actions performed by operators or operator candidates, including how procedures are to be used and executed. 


\subsection{NRC Training Parameters}

\subsection{NRC Program Descriptions and Policies}

The NRC has indicated that it is planning to maintain its current training model to train future RIs and OLEs (SECY-08-0096, as reported in NWI 2008). This current model is described succinctly in the Background section of the NRC Commission Paper SECY-08-0096 as:

"The U. S. Nuclear Regulatory Commission (NRC) has developed and implemented an effective and efficient program to train NRC inspectors and operator licensing examiners (examiners) for the current fleet of operating reactors. The NRC requires construction inspectors to complete the training and qualification program outlined in Inspection Manual Chapter (IMC) 1252, "Construction Inspector Training and Qualification Program." Reactor inspectors and examiners must complete the training and qualifications program outlined in Inspection Manual Chapter 1245, "Qualification Program for the Office of Nuclear Reactor Regulation Programs." These programs include a seven-week training regimen in either Westinghouse pressurized-water reactor or General Electric boiling-water reactor technology and use existing NRC simulators.

"The seven-week technology series includes three weeks of vendor-specific systems training, two weeks of training on transients and technical specifications, and two weeks of simulator training on normal and emergency operations. The Westinghouse full course series provides a sound basis for efficient three-week cross-training courses in Combustion Engineering and Babcock and Wilcox technologies. The cross-training courses include a mix of classroom and simulator training, and focus on the differences in systems, transient response, and technical specifications from the Westinghouse technology.

"The NRC owns four full-scope nuclear power plant control room simulators, one for each of the current United States (US) nuclear reactor vendor types. The agency acquired the simulators as "used" equipment; that is, they were originally manufactured for use by the reactor vendor for vendor-sponsored training or by utilities for licensed operator training. A staff of three simulator engineers (NRC employees) with expertise in the four hardware/software combinations maintains the NRC simulators. Three contract workers provide hardware maintenance support."

These requirements are detailed in appendices to IMC 1245 (which is referenced in the above summary description), as are requirements for refresher training. Basically, simulator refresher training is required every three years in one reactor type for which the RI or OLE is certified, alternating attendance among the vendors for which the person is certified during successive refresher training periods.

The current NRC training strategy was developed over many years, and it was not within the scope of this project to examine or evaluate the adequacy or appropriateness of this training strategy. Consequently, for the purposes of this analysis, the NRC strategy was accepted to define key parameters of the training for which the simulator fidelity requirements were being assessed. The scope of this project also 
did not include evaluation of the technical content of the various TTC courses except as a resource for validating/verifying the high-level KSA inventory and taxonomy.

The NRC has indicated that while awaiting the KSA catalogs, it has:

- developed four training courses to provide an overview of the differences between the new reactor designs (i.e., Westinghouse AP-1000; GE Nuclear Energy ABWR and ESBWR; AREVA Nuclear Power U.S. EPR; and Mitsubishi Heavy Industries [2006], Ltd U.S. APWR) and the operating reactor designs

- piloted a detailed 2-week course similar to the classroom portions of the Combustion Engineering and Babcock and Wilcox cross-training courses for the AP-1000 design

- planned to develop similar cross-training courses for the other new reactor designs and deliver them at the Professional Development Center for Office of New Reactor Licensing project managers and technical reviews.

Table 6.1 provides an example of the topics addressed in the PWR simulator training course (REF \#624). Examination of the outline shows that the trainees have the opportunity to sequentially work through 1) specific systems, 2) integrated plant operations, 3) operations in which instrument failures and other malfunctions affect the plant, and 4) emergencies requiring use of the EOPs.

\subsection{Focus Group Characterizations of Training}

\subsubsection{Instructor Characterizations}

TTC instructors described the training program as follows: The initial 7-week training program consists of 5 weeks of classroom training, followed by 2 weeks of simulator training on normal and emergency evolutions. This evolution-packed simulator training period introduces trainees to the concepts involved in integrating the information that they have learned about component operation, system operation and interactions between systems, operating procedures, technical specifications of the plant license defining the envelope within which the plant must operate, how work is apportioned among the $\mathrm{CR}$ crew, and how crew members interact to monitor and operate the plant.

The focus of the 2-week simulator training period is to teach the trainees, through hands-on operation of the simulator, how the plant is operated. The period is too short to teach fluent operation, and no attempt is made to teach evaluation activities required by their jobs - that is taught elsewhere. The emphasis is on learning by doing, and on developing an understanding and appreciation of the many facets and extent of the operator's job.

\subsubsection{The Role of On-the-Job Training}

The participants in the focus group discussion indicated that the trainees are responsible for learning through on-the-job training (OJT) at their assigned plants how the concepts they have learned in their formal training apply to their jobs. They reported that this OJT extends from learning the CR layout and control panel organization, to the position and operation of individual switches and controls, to the location and appearance of the meters and other display elements necessary to monitor and operate the 
plant. They reported that these considerations also were true for the refresher training, which involves 1 week of simulator training every 2 years for OLEs, and every 3 years for RIs. The refresher training alternates among vendor technologies for individuals who are certified on more than one reactor type.

Table 6.1. Example Scope of TTC PWR Simulator Training Course (\#624)

\begin{tabular}{|c|c|}
\hline $\begin{array}{l}\text { Duration } \\
\text { (hrs) }\end{array}$ & Primary Objective of the Module \\
\hline 1 & $\begin{array}{l}\text { Normal Ops - Demonstrate operation of CVCS, the pressurizer level control system, and the main } \\
\text { turbine generator EHC }\end{array}$ \\
\hline 1 & $\begin{array}{l}\text { Normal Ops - Demonstrate operation of the rod control system and Excore nuclear instrumentation } \\
\text { system }\end{array}$ \\
\hline 1 & $\begin{array}{l}\text { Normal Ops - Demonstrate use of general operating instructions (GOIs) and operation of reactor } \\
\text { protection system }\end{array}$ \\
\hline 4 & Normal Ops - Reactor startup and criticality \\
\hline 1 & $\begin{array}{l}\text { Normal Ops - Demonstrate operation of condensate, feedwater, auxiliary feedwater, and steam } \\
\text { generator water level control }\end{array}$ \\
\hline $1 / 2$ & Normal Ops - Demonstrate conduct of control room operations \\
\hline 1 & $\begin{array}{l}\text { Normal Ops - During steady-state power demonstrate the operation of CVCS, pressurizer level } \\
\text { control system, and main turbine generator EHC }\end{array}$ \\
\hline 1 & $\begin{array}{l}\text { Normal/Abnormal Ops - Demonstrate reactor criticality, startup, and power ascension with } \\
\text { Intermediate Range Nuclear Instrumentation Failure }\end{array}$ \\
\hline 3 & $\begin{array}{l}\text { Normal/Abnormal Ops - Demonstrate power ascension from } 30 \% \text { to } 100 \% \text { Power with pressurizer } \\
\text { level transmitter failure }\end{array}$ \\
\hline 1 & Normal Ops - Demonstrate operation of main steam and steam dump control \\
\hline 1 & $\begin{array}{l}\text { Normal Ops - Demonstrate operation of reactor coolant system and pressurizer pressure control } \\
\text { system }\end{array}$ \\
\hline $11 / 2$ & $\begin{array}{l}\text { Normal/Abnormal Ops - Demonstrate reactor power reduction from } 100 \% \text { to } 50 \% \text { power with rods } \\
\text { speed controller failure, first stage pressure failure, feed pump speed control failure, and pressurizer } \\
\text { PORV failure to open. }\end{array}$ \\
\hline 1 & $\begin{array}{l}\text { Normal/Abnormal Ops - Demonstrate power increase from } 50 \% \text { to } 100 \% \text { power with stuck rod, and } \\
\text { charging pump failure. }\end{array}$ \\
\hline 1 & $\begin{array}{l}\text { Normal Ops - Demonstrate operation of service water, component cooling water, and containment } \\
\text { systems }\end{array}$ \\
\hline $11 / 2$ & $\begin{array}{l}\text { Normal/Abnormal Ops - Demonstrate power reduction from } 100 \% \text { to } 45 \% \text { power with pressurizer } \\
\text { pressure transmitter failure and steam pressure transmitter failure }\end{array}$ \\
\hline $11 / 2$ & $\begin{array}{l}\text { Normal Ops - Demonstrate power increase from } 45 \% \text { to } 100 \% \text { power with ALL controls in } \\
\text { MANUAL }\end{array}$ \\
\hline 1 & Emergency Ops - Demonstrate operation of ECCS and ESF Status Panels \\
\hline 1 & $\begin{array}{l}\text { Normal/Abnormal/Emergency Ops - Demonstrate power increase from } 50 \% \text { to } 100 \% \text { power with } \\
\text { RTD failure, and leak in RCS letdown }\end{array}$ \\
\hline $11 / 2$ & $\begin{array}{l}\text { Normal/Abnormal/Emergency Ops - Demonstrate power increase from } 20 \% \text { to } 75 \% \text { power with } \\
\text { small leak in RCS requiring reactor shutdown }\end{array}$ \\
\hline 1 & $\begin{array}{l}\text { Normal/Emergency Ops - Demonstrate operation of electrical buses including starting/syncing } \\
\text { emergency diesel generator with buss }\end{array}$ \\
\hline
\end{tabular}


Table 6.1. (contd)

\begin{tabular}{|l|l|}
\hline $\begin{array}{c}\text { Duration } \\
\text { (hrs) }\end{array}$ & \multicolumn{1}{c|}{ Primary Objective of the Module } \\
\hline $11 / 2$ & $\begin{array}{l}\text { Normal/Abnormal/Emergency Ops - Demonstrate steady-state power operation with failure of } \\
\text { accumulator pressure and loss of one vital buss followed by loss of all offsite power }\end{array}$ \\
\hline 3 & $\begin{array}{l}\text { Normal/Abnormal/Emergency Ops - Demonstrate power reduction from } 100 \% \text { to } 50 \% \text { power with } \\
\text { steam generator tube leak requiring a reactor shutdown }\end{array}$ \\
\hline $11 / 2$ & $\begin{array}{l}\text { Normal/Abnormal/Emergency Ops - Demonstrate power reduction from } 100 \% \text { to } 75 \% \text { power with } \\
\text { steam leak and subsequent reactor shutdown }\end{array}$ \\
\hline $11 / 2$ & Abnormal/Emergency Ops - Static exam \\
\hline 1 & Normal Ops - Demonstrate mid loop operation, bubble formation, and RCS heat up \\
\hline 35 & TOTAL HOURS \\
\hline
\end{tabular}

\subsubsection{Importance of Trainee Interactions with the Plant}

One additional consensus position was advanced in the focus group discussion: To better their knowledge of the concepts learned during training, trainees need to actually interact with the plant controls, although physical fidelity of the controls used for operation is not required.

Thus, for instance, in the PWR course outlined in the previous section, the demonstration activities identified are actually carried out with the trainees at the controls working through the evolutions under the guidance of the instructors.

\subsubsection{Experience and Background of Refresher Trainees}

Additional insights regarding the requalification refresher course were obtained in a focus group that included several TTC instructors. In this discussion, the DIF analysis planned for application to the Task/KSA Inventory was described to obtain suggestions on the adequacy, appropriateness and completeness of the plans. Although no significant criticisms or suggestions were offered, the discussions ranged widely and touched on the training program objectives, the knowledge and experience levels of the target group to be trained, the physical fidelity of controllers and displays vs. flat panel presentations with touch-screen controls, and the experience levels of individuals coming to the refresher simulator training. Regarding the refresher course, it was emphasized that the audience included a wide variety of experience levels, and that attendance was required for people to maintain certification in that reactor type. The attendees may not have job responsibilities like those of OLEs and RIs during the intervening period. A transcript of this discussion is included in Appendix A.

\subsubsection{Overall Training Objectives}

A question about training objectives elicited a lively discussion (roughly transcribed in Appendix A) that resulted in general agreement that the objective of the simulator training is to teach the trainees enough about actual plant operation that they can evaluate it. Regarding fidelity, the focus group participants agreed that enough physical fidelity is needed so they can work through plant operations. They start with simple start-up and power maneuvering operations, and then move to transient and off-normal events 
that require the use of EOPS. Working through this sequence gives the trainees a good idea of what is involved in performing these tasks. They reiterated that they needed this understanding in order to evaluate the performance of operators at their assigned plants.

\subsection{Demographics of NRC Staff that Affect Training}

\subsubsection{Staff Turnover and Expansion}

Changing demographics pose difficult challenges for the certification and regulation of NPPs in the United States. According to the U.S. Government Accounting Office (GAO) (2007:48), an estimated $29 \%$ of NRC staff will be eligible to retire in 2009 , and the percentage will increase to $37 \%$ by 2011 . To replace outgoing staff and manage growing review and licensing workloads, the NRC projects that it will need to hire and train almost 4,000 new employees. This need equates to approximately 300 hires per year until at least 2010 (GAO 2007:2). In reviewing the NRC's ability to meet this demand, the GAO estimates that the nuclear industry, which will require an estimated 90,000 workers through at least 2011 (p. 37), will be competing with the NRC for workers. They estimate that this competition will challenge the NRC's ability to recruit the necessary new staff. The pool of available talent, which significantly decreased during the 1980s and 1990s, currently is small, although the pool is now expanding as a consequence of steadily growing undergraduate and graduate enrollment and graduation rates (Wogman et al. 2005:138; Hamlin 2009). Experts project that the number of prospective qualified employees will be insufficient to meet the demand. Demand for qualified employees is estimated to exceed supply by $150 \%$, which translates to unmet demand by the NRC ranging from 40 individuals during the best years to over 100 individuals by 2011 .

In 2006, the GAO reported that the NRC successfully closed 55 critical skill gaps by hiring 371 new employees. However, 155 additional critical skill gaps still remained. The GAO also concluded that the NRC had made good use of flexibilities for critical skill transfer, but it must continue to study the potential workload and staffing requirements for the estimated $20 \mathrm{COL}$ applications and the operation of 29 new NPPs. Despite these challenges, the GAO concluded that the NRC has been generally effective in managing its current and future human capital requirements, but that it must continue to study the workload demands and critical skill gaps that will arise with the anticipated increase in licensing workload and demographic shifts (GAO 2007). Data assembled by the GAO indicate that the NRC will face a significant demand for training in the upcoming years, and that the large number of new employees needing training is likely to have less experience than those hired in the past, particularly in the nuclear industry.

\subsubsection{Years and Type of Experience of On-Line Survey Respondents}

The on-line survey was administered to all current NRC RIs and OLEs in mid-2008. Ninety-seven individuals submitted completed surveys. Consistent with nuclear industry patterns, most of the RI/OLE survey respondents had many years of experience in the current jobs they held at the time of the survey. ${ }^{1}$ The overall average number of years of experience for this cohort in their current job was 6.6 years. One

\footnotetext{
${ }^{1}$ There are probably multiple explanations for this survey finding. One likely explanation is the nuclear industry's rapid growth in the 1960s and 1970s, which attracted new personnel into the industry. Until recently, growth in the nuclear industry had been stagnant.
} 
incumbent had 28 years of experience as an RI; another had 22 years of experience as an OLE. The distribution of respondents in terms of years in current position is shown in Figures 6.1 and 6.2.

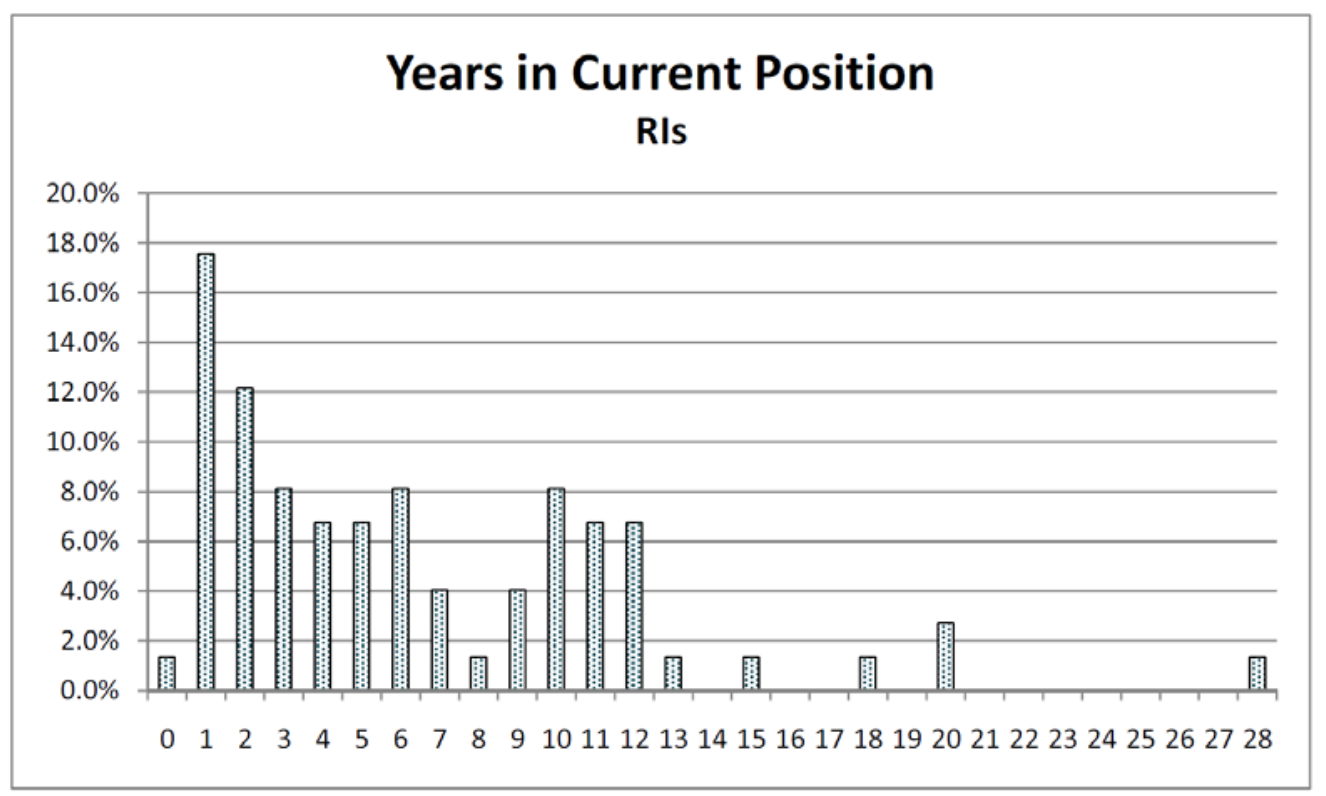

Figure 6.1. Years in Current Position - RIs

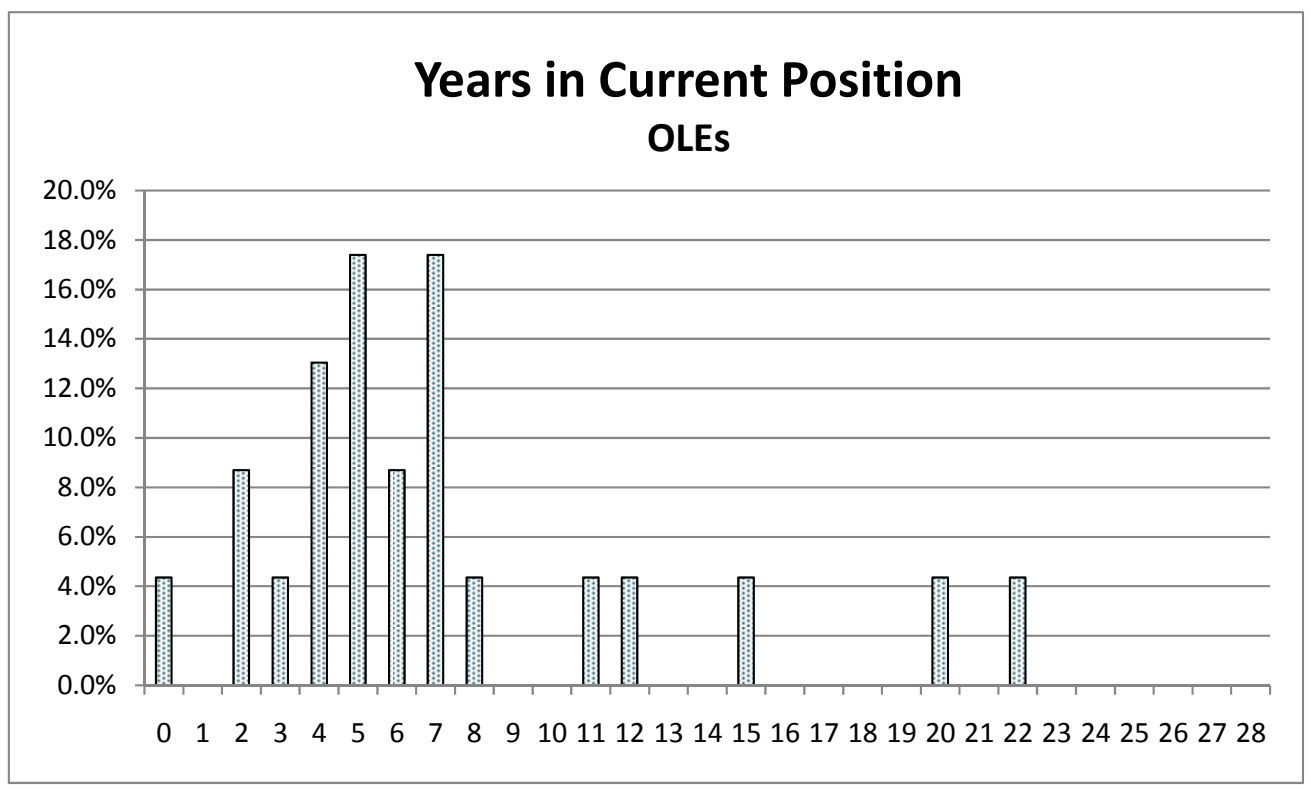

Figure 6.2. Years in Current Position - OLEs

Each respondent also was asked to report on their previous work experience, and how long they had held each position. As shown in Figures 6.3 and 6.4 and in Table 6.2, it is clear that both OLEs and RIs have had substantial additional experience, both in terms of having held multiple jobs and in terms of the time spent in each position. Over $40 \%$ of the OLEs responding to the survey reported previous experience as an RI. About 13\% of RI's responding to the survey reported previous experience as an 
OLE. Over $50 \%$ of OLEs and approximately $25 \%$ of RIs reported previous employment as a Senior Reactor Operator, and both groups of respondents reported significant time spent in other nuclear industry positions prior to their current work. The range of experience was broad, both in current and previous positions.

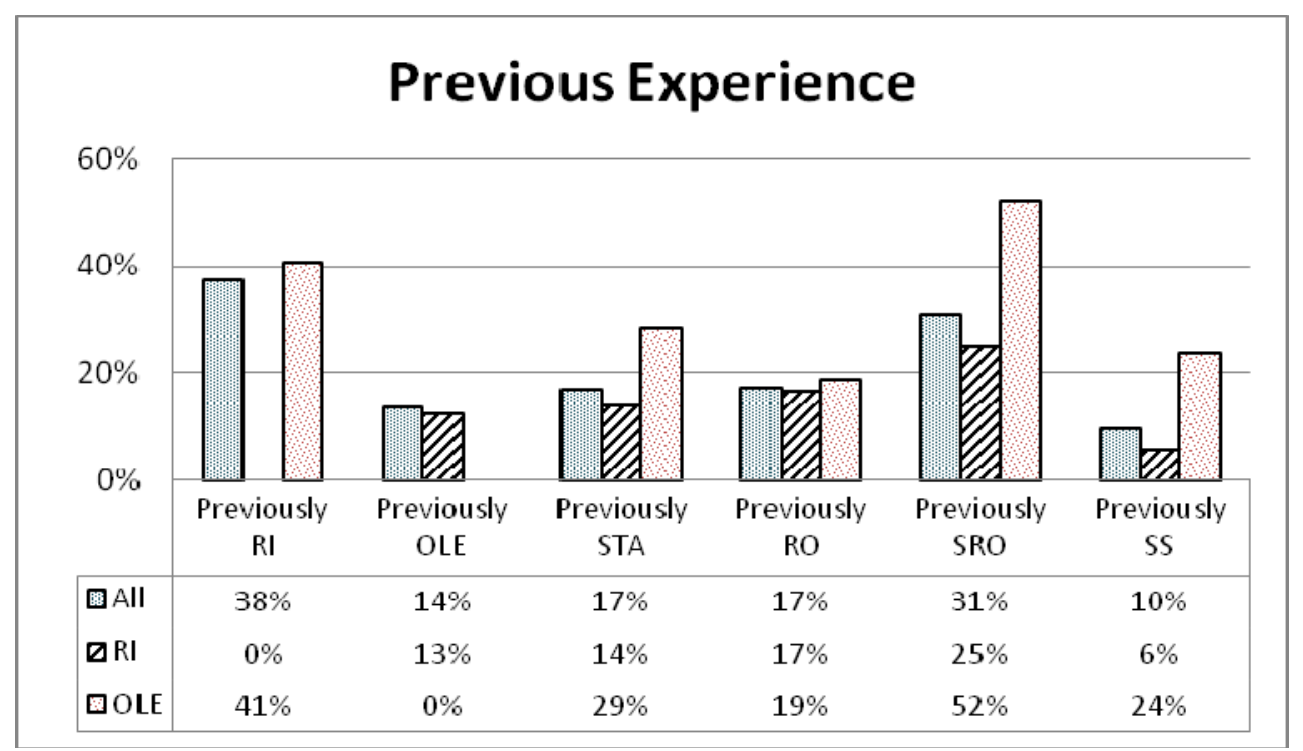

STA=Shift Technical Advisor; RO=Reactor Operator; SRO=Senior Reactor Operator; SS=Shift Supervisor

Figure 6.3. Previous Experience - All Respondents, RIs and OLEs

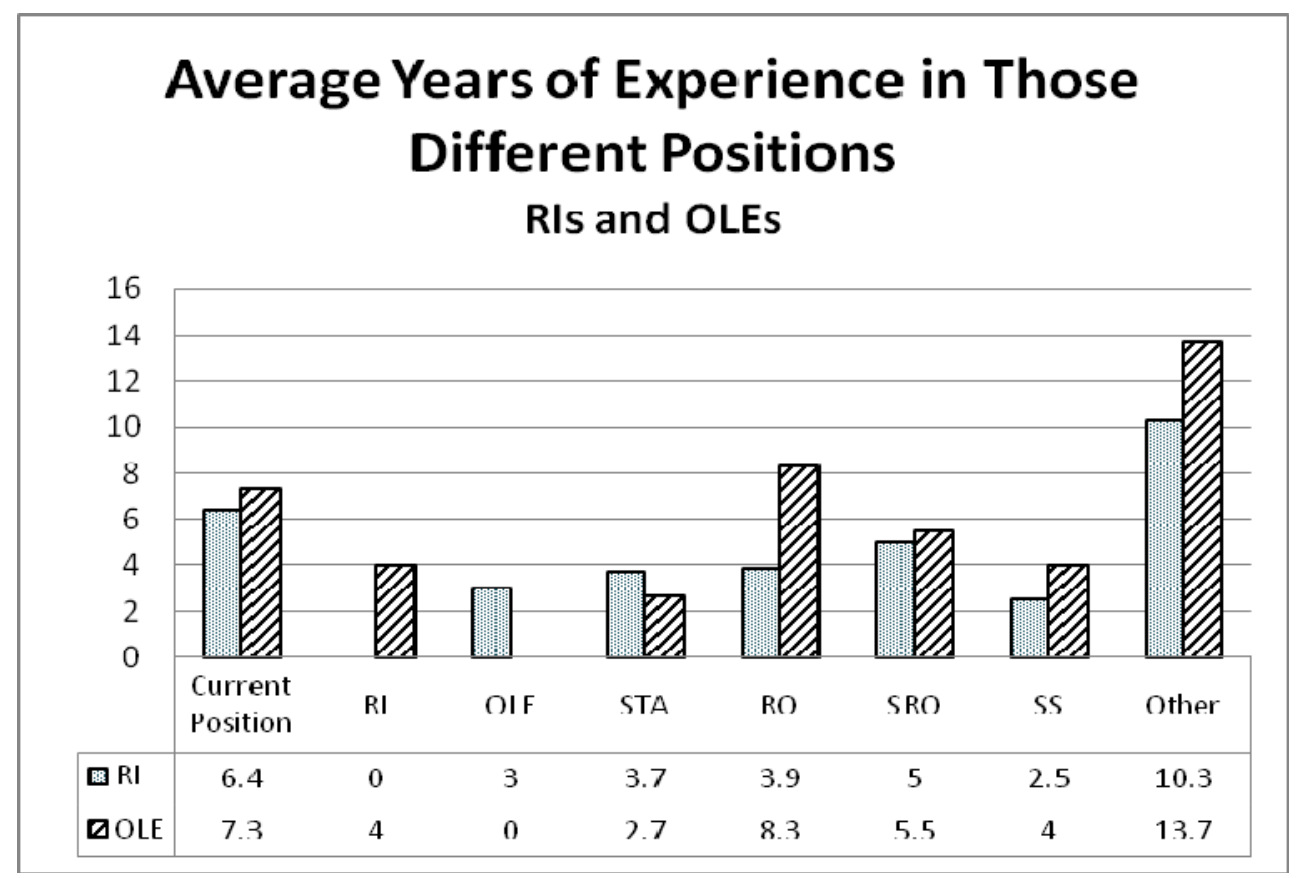

STA=Shift Technical Advisor; RO=Reactor Operator; SRO=Senior Reactor Operator; SS=Shift Supervisor

Figure 6.4. Average Years of Experience in Different Positions - RIs and OLEs 
Table 6.2. Range of Years of Experience in Current and Previous Positions

\begin{tabular}{|l|c|c|}
\hline \multirow{2}{*}{\multicolumn{1}{|c|}{ Position }} & RI Respondents & OLE Respondents \\
\cline { 2 - 3 } & \multicolumn{2}{|c|}{ Range of Experience (Years) } \\
\hline Current Position & $0-28$ & $0-22$ \\
\hline Other Positions & & $1-12$ \\
\hline RI & NA & NA \\
\hline OLE & $1-8$ & $1-6$ \\
\hline Shift Technical Advisor & $1-8$ & $2-20$ \\
\hline Reactor Operator & $1-8$ & $2-20$ \\
\hline Senior Reactor Operator & $1-16$ & $1-5$ \\
\hline Shift Supervisor & $1-4$ & $3-22$ \\
\hline Other (related) & $2-26$ & \\
\hline
\end{tabular}

\subsubsection{TTC Training Experience of On-Line Survey Respondents}

OLEs and RIs were asked to respond to questions regarding their current assignment, TTC classroom training, and TTC simulator training by reactor type (vendor) and whether they had received general and/or simulator training by the manufacturer in question, and how many refresher courses they had received on each reactor type. They also were asked how many years it had been since their last TTC simulator training class.

As shown in Figures 6.5 and 6.6, the survey data indicate that, as expected, OLEs have been trained for and have experience working in a greater range of reactor types than RIs.

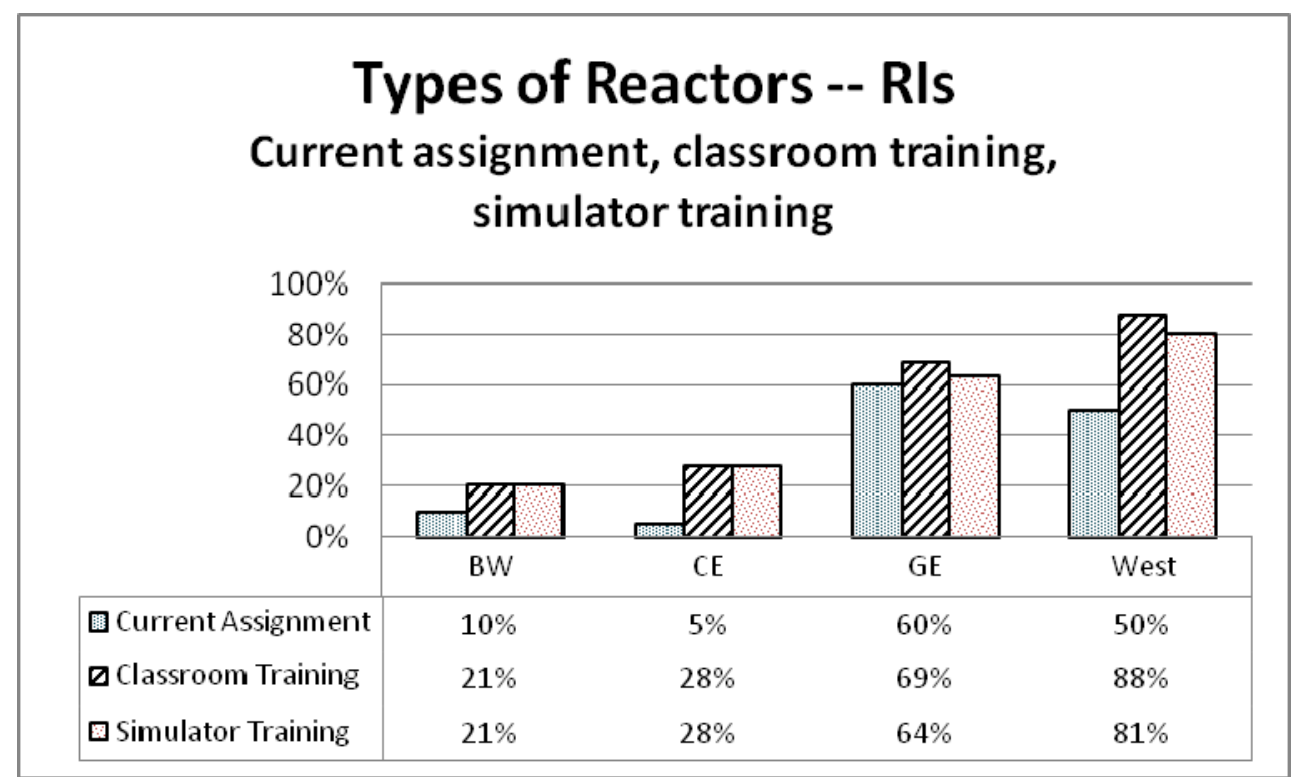

$\mathrm{BW}=$ Babcock and Wilcox; $\mathrm{CE}=$ Combustion Engineering; $\mathrm{GE}=$ General Electric; West=Westinghouse

Figure 6.5. Types of Reactors of Current Assignment and Classroom and Simulator Training - RIs 


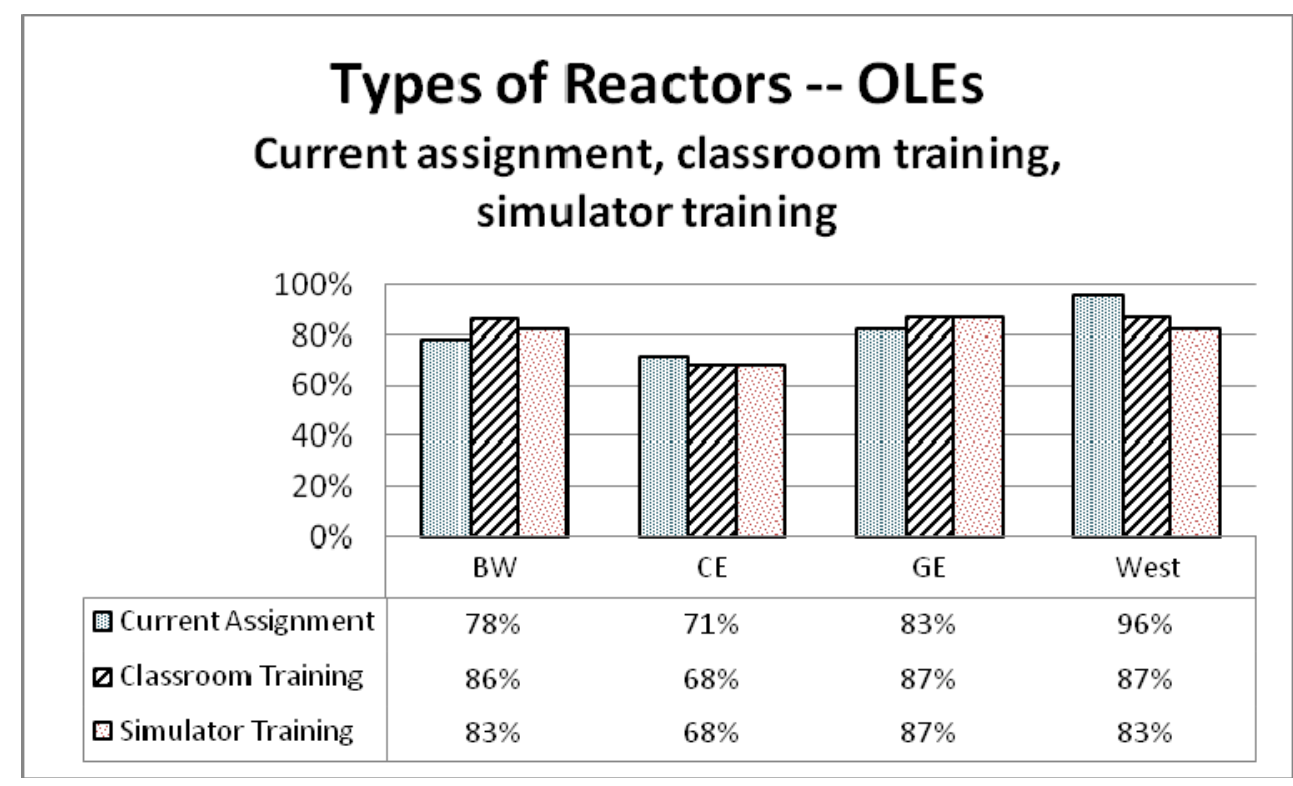

$\mathrm{BW}=$ Babcock and Wilcox; $\mathrm{CE}=$ Combustion Engineering; $\mathrm{GE}=$ General Electric; West=Westinghouse

Figure 6.6. Types of Reactors of Current Assignment and Classroom and Simulator Training - OLEs

Each OLE reported being trained for and having experience with multiple technologies simultaneously and having received refresher training on a regular or semi-regular basis.

As seen in Figure 6.7, almost $75 \%$ of the respondents reported that they had received TTC refresher training relevant to the $\mathrm{CR}$ or simulator at their assigned location(s). Figure 6.8 shows the number of simulator training courses the RIs and OLEs responding to the on-line survey had received for each reactor type. The greatest proportion of respondents reported having attended one simulator refresher training, although about a third reported having attended two or more refresher courses.

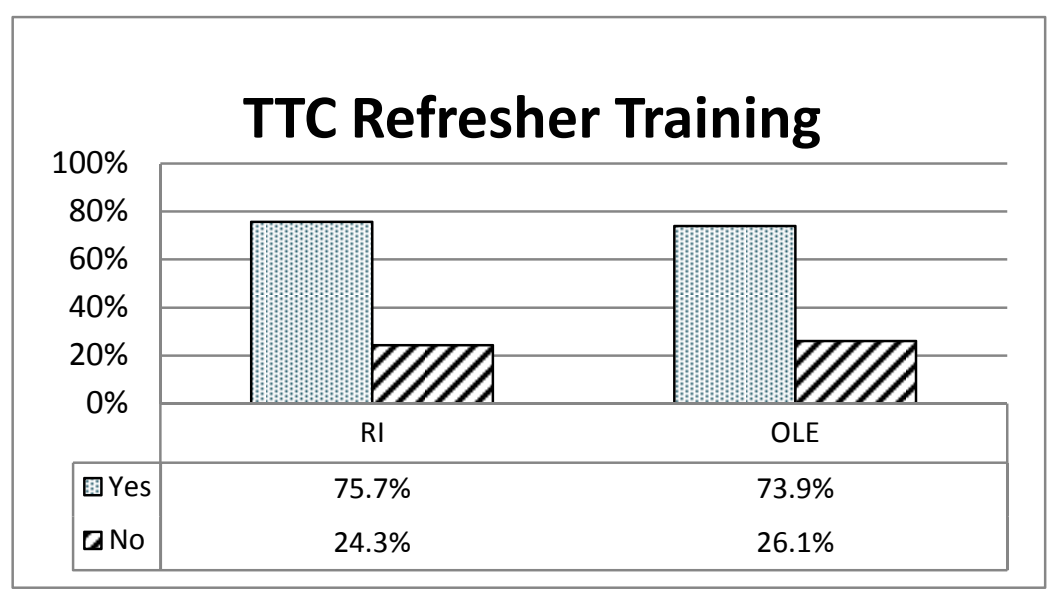

Figure 6.7. Proportion of RIs and OLEs Who Have Had TTC Refresher Training 


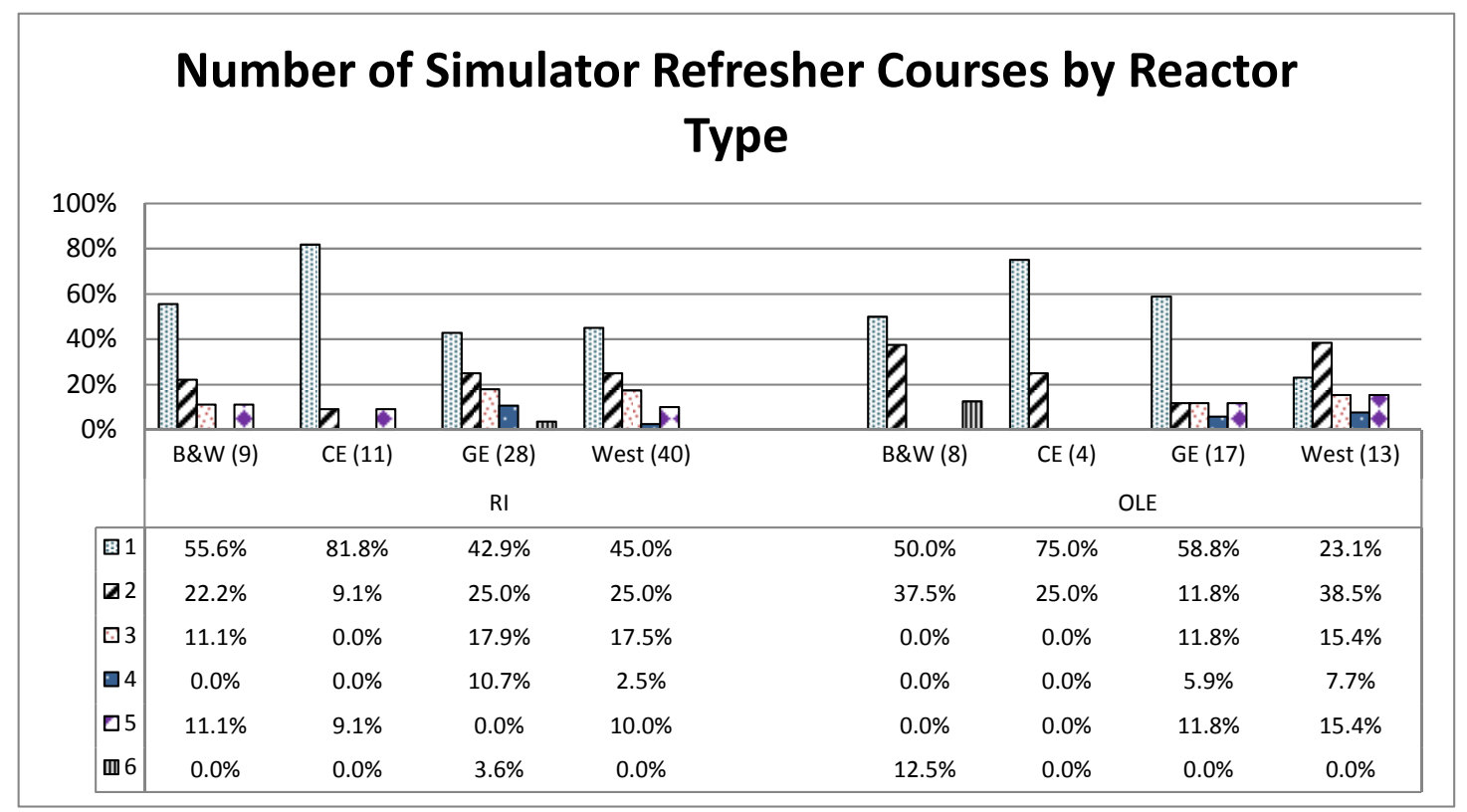

$\mathrm{BW}=$ Babcock and Wilcox; $\mathrm{CE}=$ Combustion Engineering; $\mathrm{GE}=$ General Electric; West=Westinghouse (Numbers in parentheses are the number of respondents.)

Figure 6.8. Number of Simulator Refresher Courses by Reactor Type - RIs and OLEs

Figure 6.9 shows a distribution of the number of years since RIs and OLEs last received simulator training. Nearly $75 \%$ of RIs and over $90 \%$ of OLEs responding to the survey reported receiving simulator training within the last 2 years. Almost $70 \%$ of OLEs reported receiving simulator training with the last year.

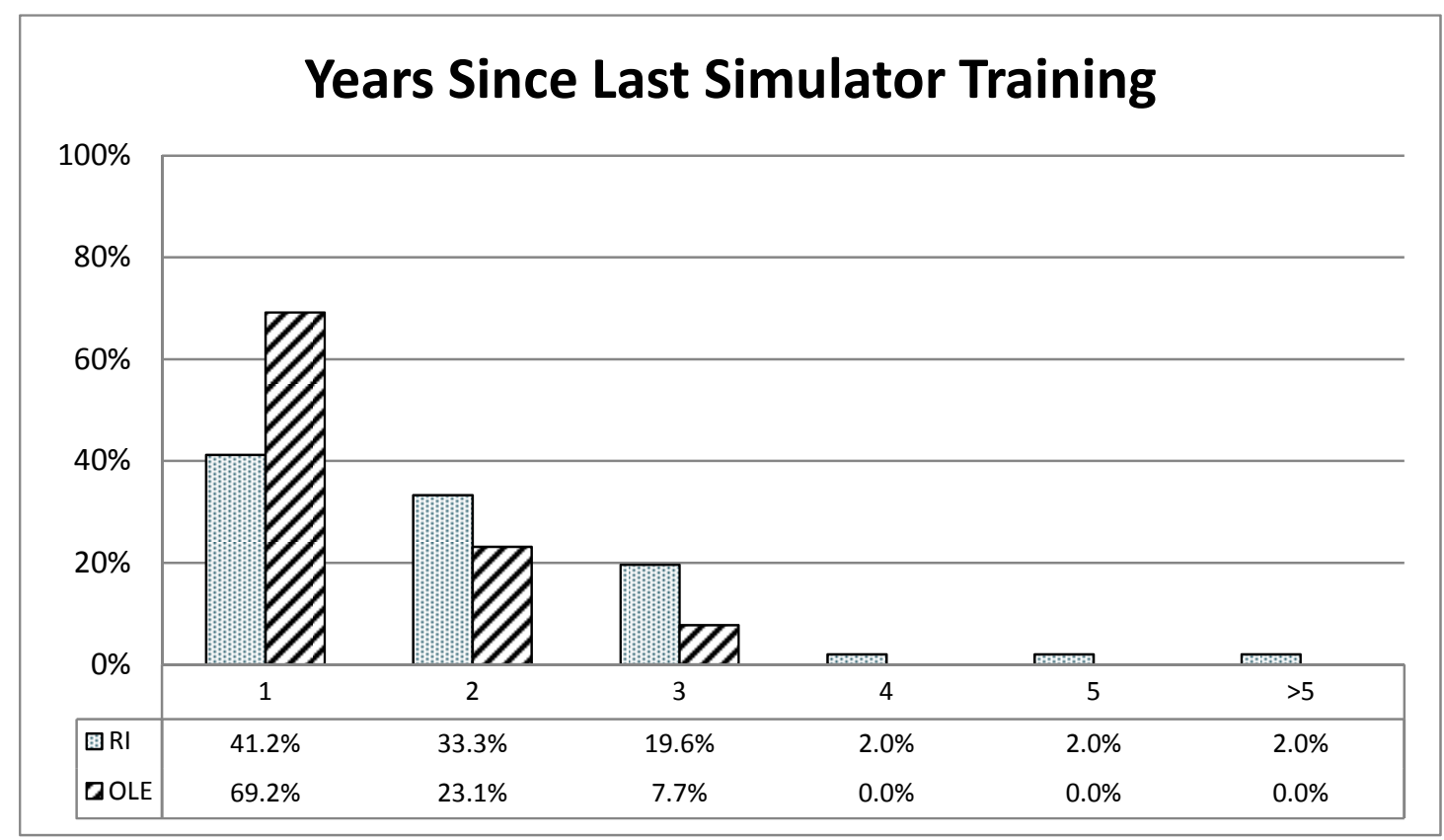

Note: Rounded to the nearest year (6 months rounded down)

Figure 6.9. Years Since Last TTC Simulator Training - RIs and OLEs 


\subsubsection{Familiarity with New Control Room Technologies}

Survey respondents were asked to indicate their familiarity with new CR technologies, including graphical user interfaces, group-view displays, soft controls, computer-based procedure systems, and electronic messaging. As shown in Figure 6.10, both RI and OLE respondents reported greatest familiarity with electronic messaging and graphical user interfaces, and least familiarity with soft controls, computer-based procedure systems, and computer-based operating systems. RIs generally reported less familiarity with these new technologies than OLEs. Seventy-nine percent of responding RIs rated their familiarity with soft controls as a 1 or 2 on the 5-point familiarity scale (i.e., 1 =unfamiliar to $5=$ very familiar), compared to $59 \%$ of responding OLEs. Seventy-five percent of RIs and $71 \%$ of OLEs gave a 1 or 2 rating to their familiarity with computer-based operating systems; and $70 \%$ of RIs and $71 \%$ gave similar ratings for computer-based procedure systems.

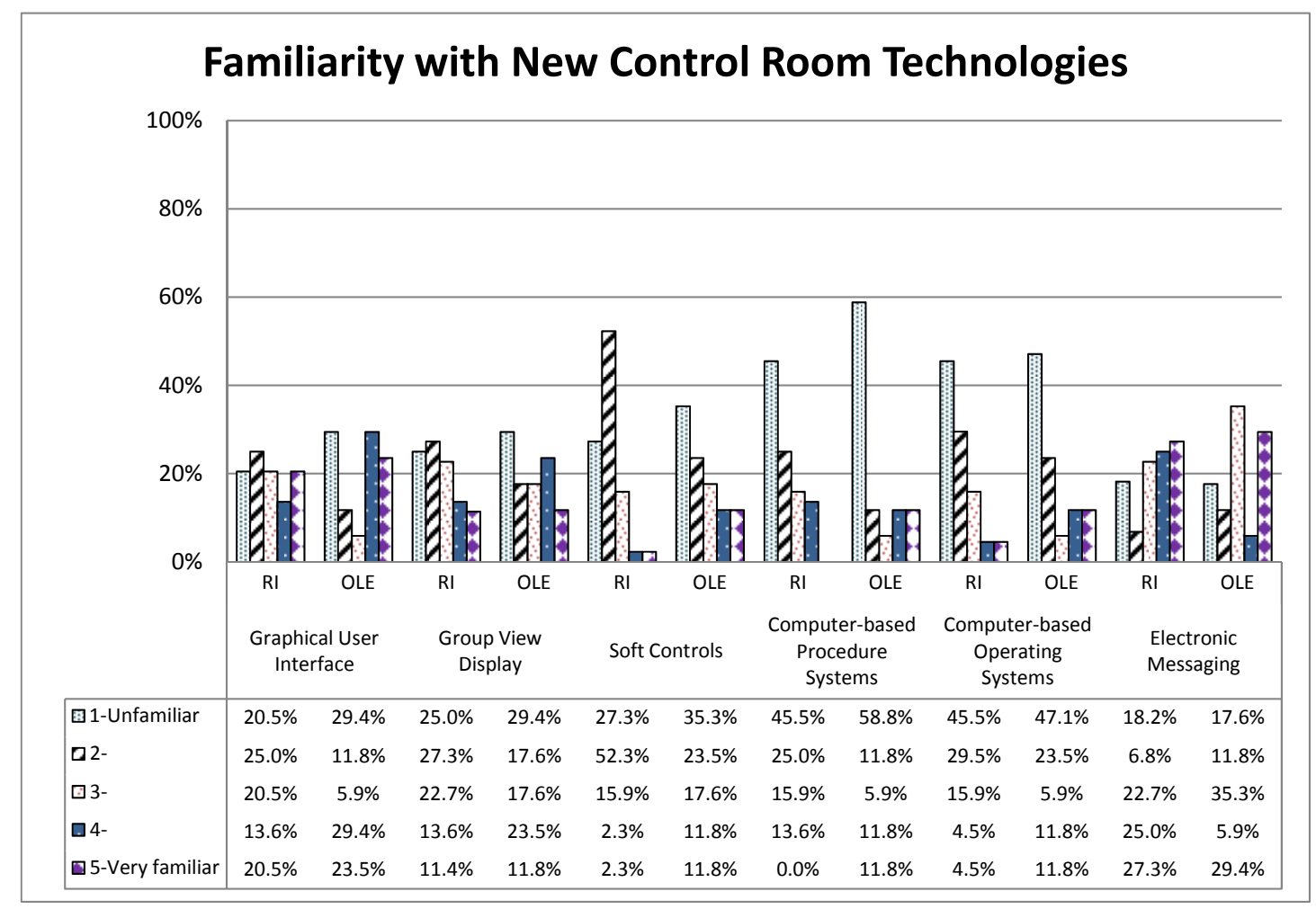

$\mathrm{N}=44 \mathrm{RI} ; 17$ OLE

Figure 6.10. Familiarity with New Technologies - RIs and OLEs

\subsubsection{On-Line Survey Respondents' Evaluation of Current TTC Simulator Training}

The survey respondents were asked to evaluate their simulator training in terms of its value to performance of their job responsibilities. Their evaluations were based on the following criteria:

- 1 - Counterproductive

- 2 - Unnecessary 
- 3 - Positive impact but too little value

- 4 - Valuable/worthwhile

- 5 - Essential in developing their knowledge in each of three areas.

As shown in Figure 6.11, most survey respondents rated the simulator training positively (entering a score of 4 or 5). Ninety-five percent of responding OLEs and $90 \%$ of RIs rated the training either valuable/worthwhile or essential for developing their knowledge in nuclear facility operations, including system performance and interrelationships (i.e., "systems" in the Figure 6.11). The average rating by OLEs and RIs were 4.5 and 4.1 (out of 5), respectively. Over 50\% of OLEs responding to the survey rated the simulator training essential for developing this knowledge.

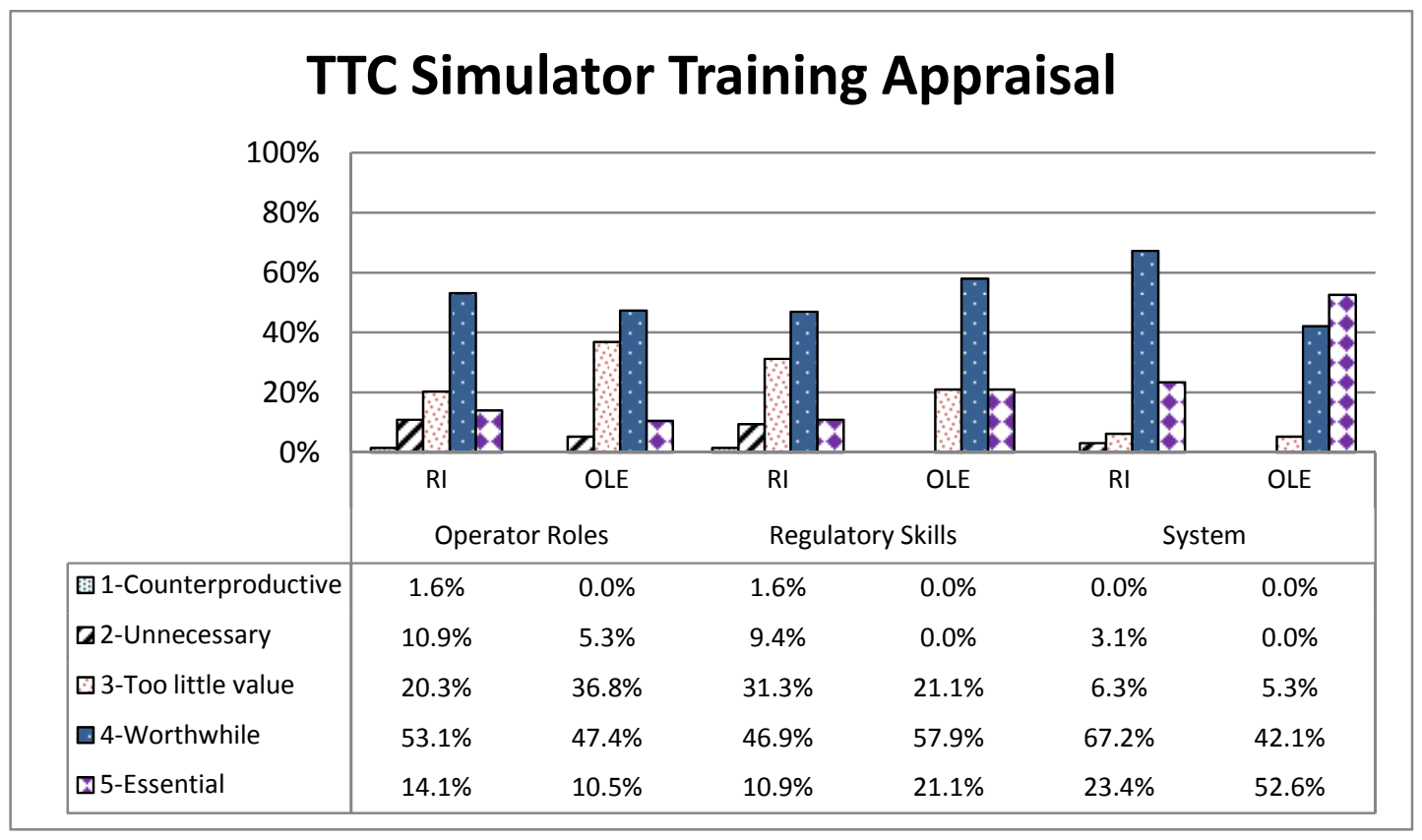

$\mathrm{N}=64 \mathrm{RI} ; 19$ OLE

Figure 6.11. Evaluation of Simulator Training Effectiveness - RIs and OLEs

Respondents' ratings of the value of simulator training for developing knowledge of facility operator's roles were slightly less positive, with $67 \%$ of RIs and $58 \%$ of OLEs rating the training as valuable/worthwhile or essential. RIs were somewhat more negative about the simulator training in terms of developing knowledge of facility operator roles than OLEs, with $2 \%$ of the RIs rating the training as counterproductive (compared to none of the OLEs) and 11\% of the RIs rating it as unnecessary (compared to $5 \%$ of the OLEs).

When asked if the simulator training was essential to developing the regulatory skills necessary for administering, documenting, and justifying a license evaluation of operator and crew performance, 58\% of the RIs and $79 \%$ of the OLEs responding to the survey rated the training as valuable/worthwhile or essential. None of the OLEs rated the training as counterproductive or unnecessary for developing these skills; $2 \%$ of the RIs rated the training as counterproductive and $9 \%$ rated it as unnecessary to developing these regulatory skills. 
In response to the question "How might the TTC simulator training be changed so that it is more beneficial to your success in performing your job responsibilities?," seven respondents commented that the training is more from the perspective of an operator than an examiner or inspector and that changing this perspective could help. One respondent opined that 2 weeks of training every 3 years is not enough training or is held too infrequently to be effective.

\subsection{Implications for Physical Fidelity of TTC Simulators in Advanced Reactor Training}

The information gathered and analyzed by the project team through review of documents, focus group discussions, and the on-line survey support the conclusion that the current training program, while short in duration, is effective in preparing initial trainees for OJT, and provides useful refresher for experienced personnel. The project team identified no information indicating that the NRC is planning a fundamental change in either the duration or approach to simulator training for RIs or OLEs. Consequently, the project team has assumed that the TTC simulator training for future RIs and OLEs will remain as a relatively short (approximately 2-week) course, that it will focus on training RIs and OLEs for reactor design families rather than a specific reactor, and that it will continue to be part of a training program that includes self-study, classroom, and OJT. The current schedule for refresher training also is expected to remain unchanged.

RI and OLE survey responses, in conjunction with other data on the demographics of NRC and other nuclear industry personnel, indicate that the current workforce has diverse and extensive experience. Many of the RIs and OLEs responding to the on-line survey reported previous experience in other relevant positions. However, the survey respondents reported limited familiarity with new CR technologies, particularly soft controls and computer-based procedure and operating systems. Forecasts of workforce demographics indicate that future NRC employees may have more limited nuclear-industry experience, and it is not clear how these future employees and experience by current employees outside power plant CRs will affect workforce familiarity with the new technologies, which are expected to play important roles in advanced reactor CRs and simulators.

Survey respondents overwhelmingly rated the TTC simulator training they received as worthwhile/ valuable or essential for developing their knowledge about nuclear facility operations. They were somewhat less positive, although still positive, about the value of the training for developing knowledge of facility operator's roles and the regulatory skills necessary for administering, documenting, and justifying a license evaluation of operator and crew performance. The survey and focus group discussions revealed that a primary goal of simulator training is to teach general concepts that can be applied across reactors in the same family. 


\subsection{Physical Fidelity Differences between the TCC Simulator and Work Location Control Rooms and Simulators and Their Impacts}

\subsection{Physical Fidelity Differences of HSI Systems}

The on-line survey of NRC RIs and OLEs contained a set of questions addressing the physical fidelity of TTC simulators and the impact of physical fidelity on job performance and difficulty of learning during refresher training. The 97 survey respondents (74 RIs and 23 OLEs) were asked to rate the physical fidelity of TTC simulator's components on a scale from 1 (no difference) to 5 (entirely different) relative to the CR (RIs) or simulators (OLEs) at their assigned locations. Respondents were asked to compare a TTC simulator to a corresponding CR or simulator of the same reactor vendor at a site to which they were "currently" (at the time the respondent was answering the questions) assigned. If a respondent reported a difference, they were then asked to rate how large an impact the difference had on job performance on a scale from 1 (no impact) to 5 (high impact). If a respondent reported a difference and they had also previously received refresher training on the same TTC simulator, then they were also asked what the impact of the differences were on the difficulty of learning course lessons during refresher training.

Figures 7.1, 7.2, and 7.3 present the ratings on fidelity differences by the entire group of respondents, RIs and OLEs, respectively. Looking across the systems, and then across the positions, reveals that few respondents reported "no difference" between the TTC simulator and their assigned CR or simulator. The CR environment received the highest percentage of "no difference" ratings from both RI and OLE respondents (12.2\% and $21.7 \%$, respectively), followed by procedures ( $10.8 \%$ for RIs and $8.7 \%$ for OLEs) and alarms/annunciators (9.5\% for RIs and $13.0 \%$ for OLEs). Computer displays, procedures, panels, and CR environment received the highest "entirely different" ratings from RIs; computer displays, procedures, and alarms/annunciators were rated "entirely different" most often by OLEs. Over half of the RI respondents gave computer displays and panels a rating of 4 (very different) or 5 (entirely different), and over half of the OLE respondents gave computer displays and procedures these high difference ratings.

\subsection{Physical Fidelity Difference Impacts on Job Performance}

Figures 7.4 and 7.5 show the ratings RI and OLE respondents to the on-line survey gave to the impact of the physical fidelity differences between the TTC simulator and the CR or simulator at their assigned location on job performance. The ratings range from 1 (no impact) to 5 (high impact). Both RIs and OLEs indicated that the impact of physical fidelity differences in the CR environment were low (67\% of RIs and $72 \%$ of OLEs indicating "no impact"). Few RIs or OLEs indicated that the physical fidelity differences of any systems caused "high impact" (score of 5) or rated the impact a 4, although 14.3\% of OLEs rated the impact of differences in procedures on job performance as either a 4 or 5 . A higher percentage of OLEs rated systems as 3 in terms of difference and 3 in terms of impact of the difference on job performance than RIs did. 


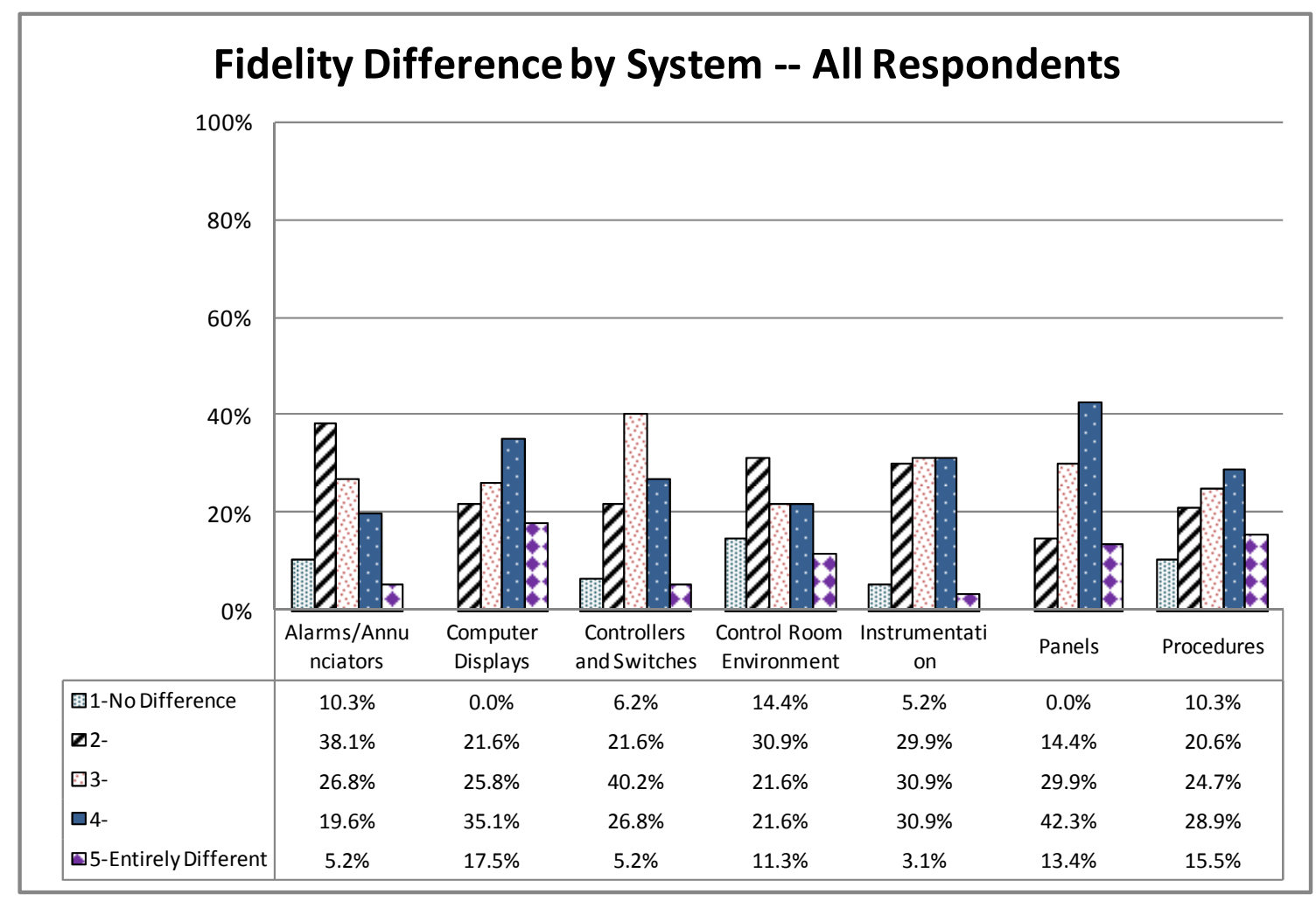

Figure 7.1. Fidelity Difference Ratings for All Systems: All Respondents

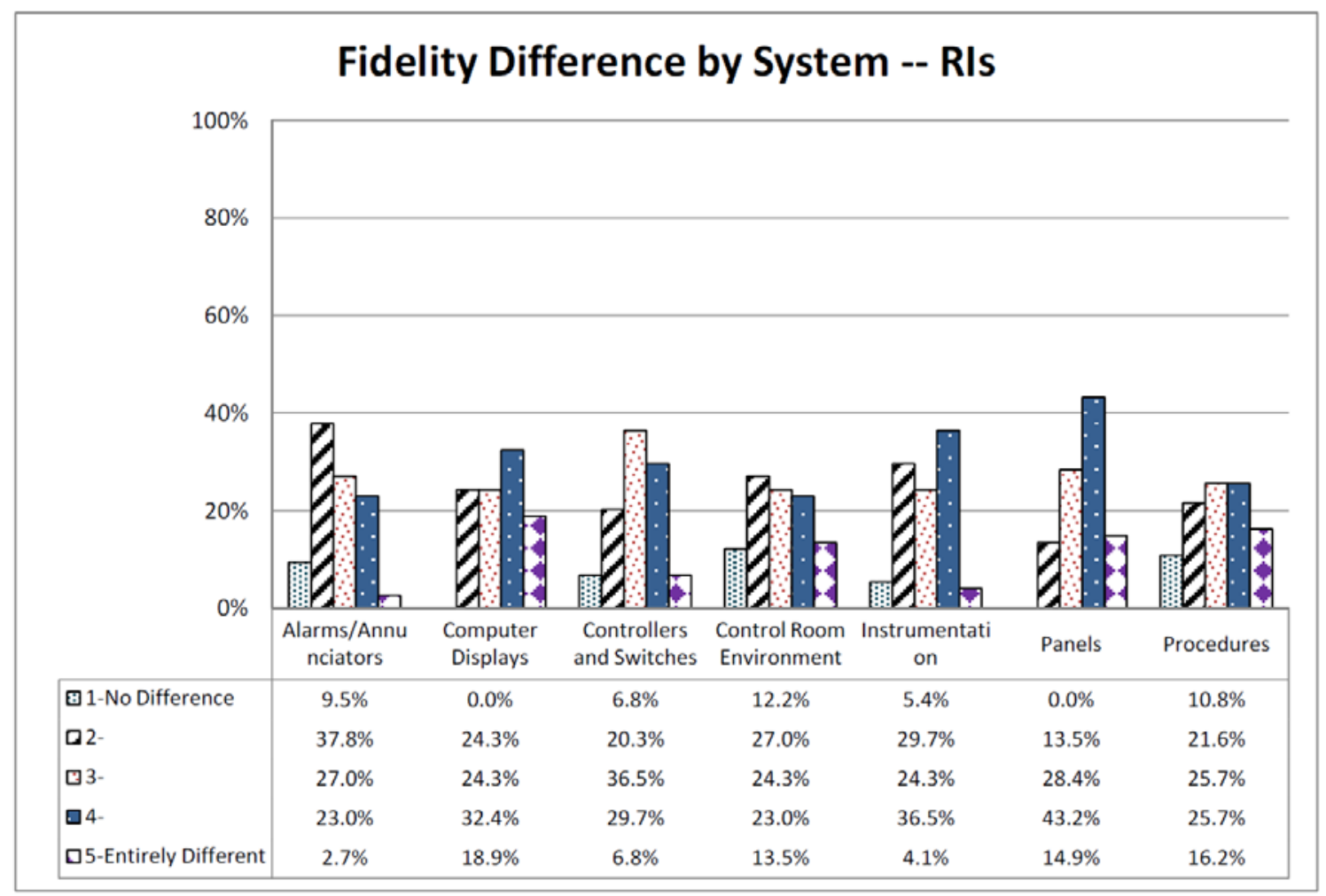

Figure 7.2. Fidelity Difference Ratings for All Systems: RIs 


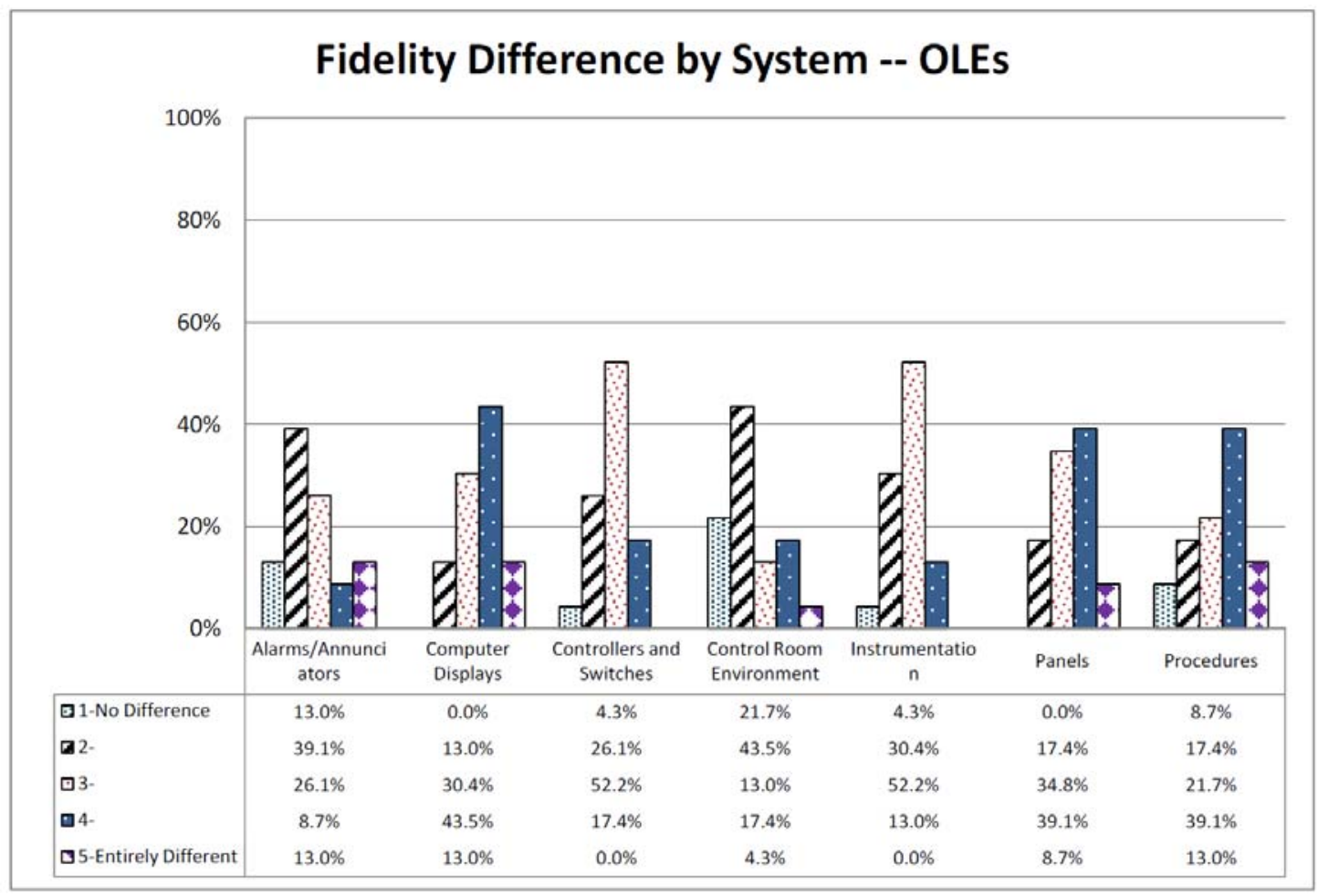

Figure 7.3. Fidelity Difference Ratings for All Systems: OLEs

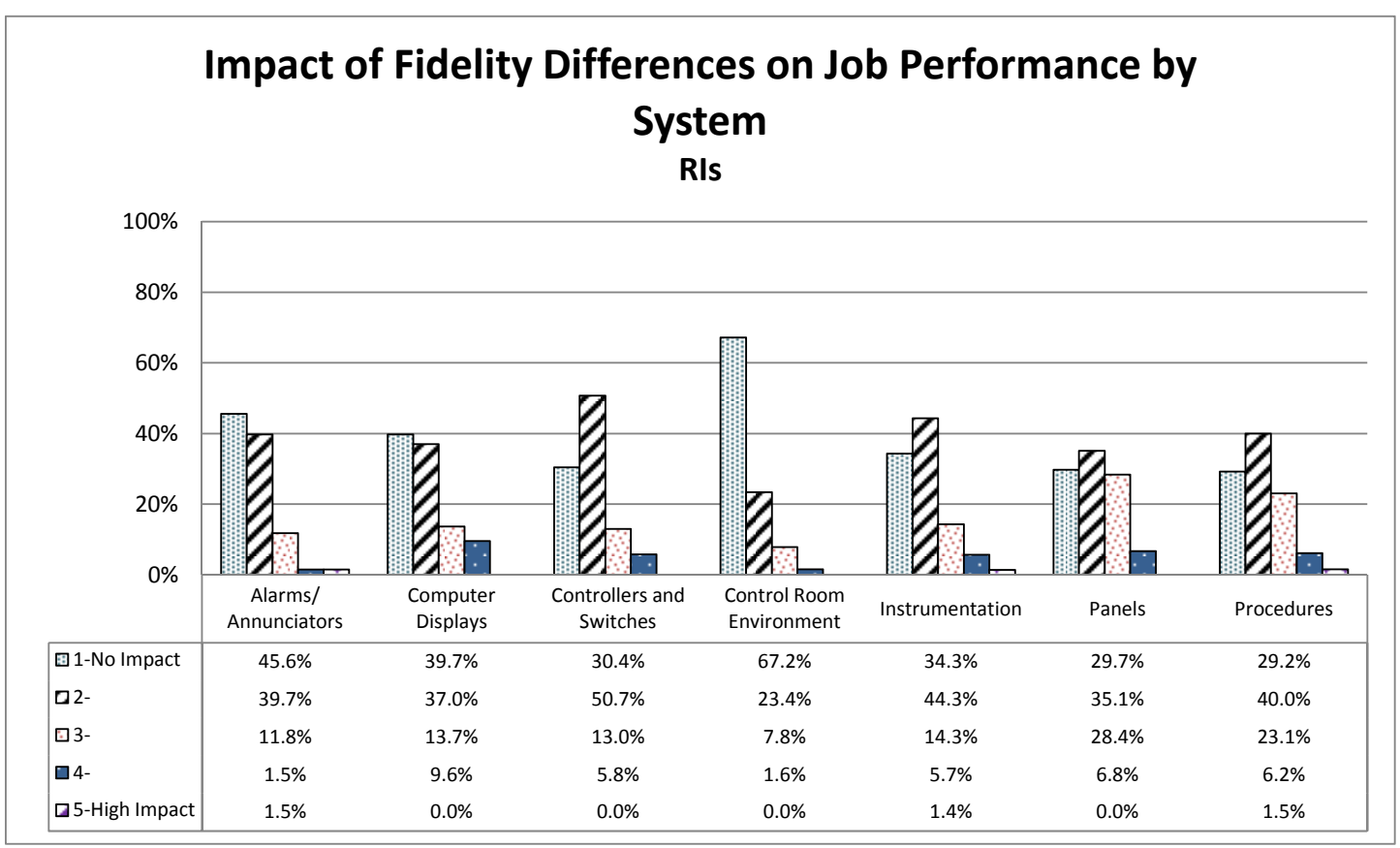

Figure 7.4. Fidelity Difference Impacts on Job Performance by System - RIs 


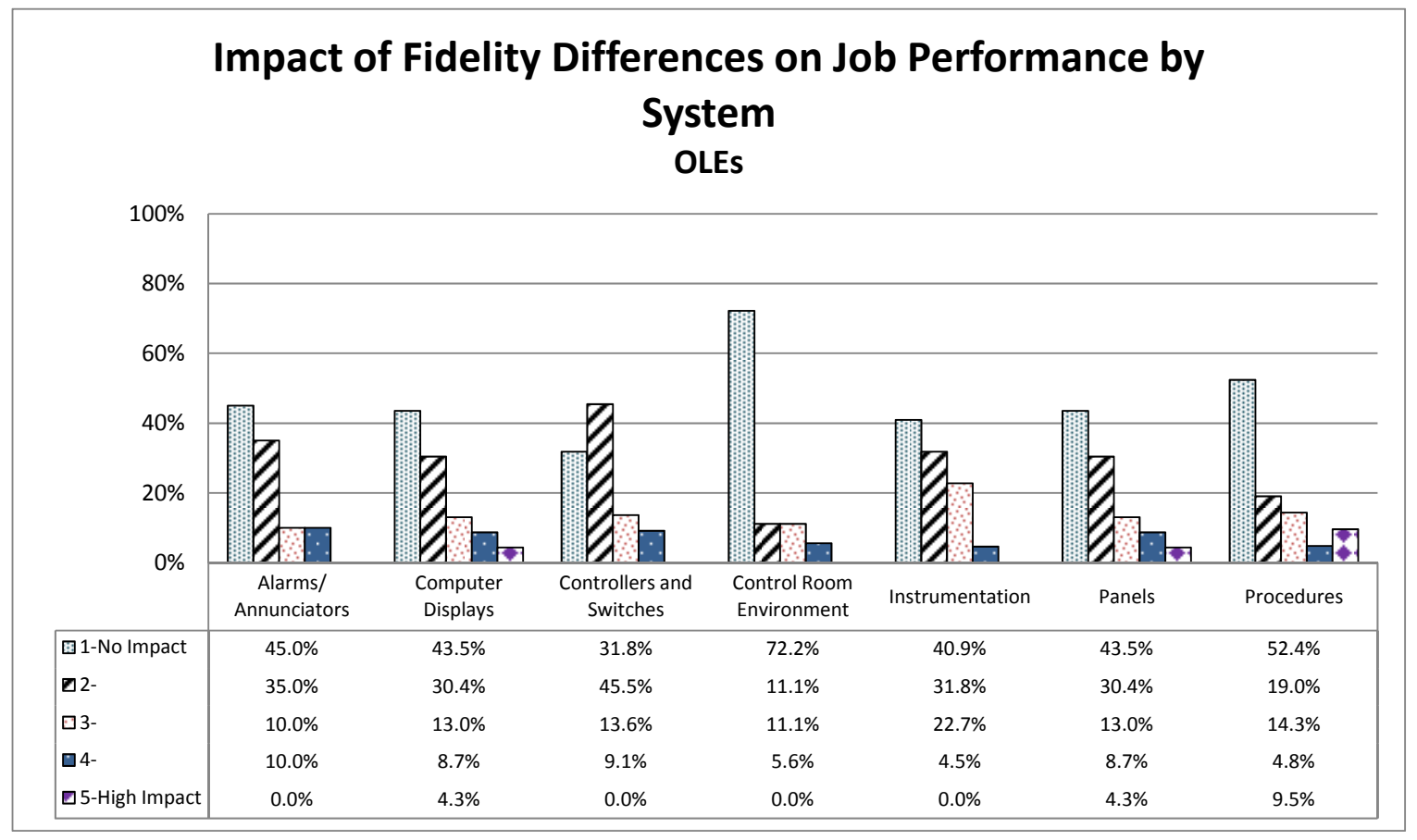

Figure 7.5. Fidelity Difference Impacts on Performance by System - OLEs

\subsection{Impact of Physical Fidelity Differences on Difficulty of Learning}

Figures 7.6 and 7.7 show the ratings of RI and OLE respondents to the on-line survey regarding the impact of physical fidelity differences between the TTC simulator and the CR/Simulator at their assigned location on learning during refresher training at the TTC. Only the 56 RIs and 17 OLEs who had received refresher training at the TTC were asked the questions about impact on difficulty of learning. As seen in these figures, the ratings by RI and OLE respondents are similar. As seen in these figures, both RI and OLE respondents rated the impact of physical fidelity differences on difficulty of learning as low for all systems. Across systems of the impact of fidelity differences of learning, the average rating by RI respondents is 1.8 and 2.2 for OLE respondents. RI respondents rated fidelity differences of no system as having a "high impact" on difficulty of learning. A small percentage of OLE respondents rated fidelity differences of procedure and computer display systems as having a "high impact" on difficulty of learning.

\subsection{System by System Ratings of Differences and Impacts}

\subsubsection{Alarms/Annunciators}

The average rating of physical fidelity difference between the TTC simulator and the $\mathrm{CR} /$ simulator at the assigned location for alarms/annunciators was 2.7 (on the 5-point scale) for RI, OLE, and all respondents. RI and OLE respondents exhibited very similar response patterns in the 1-3 score ratings. Approximately $25 \%$ of all respondents rated alarms/annunciators as 4 or 5 , with respect to simulator fidelity differences. A higher percentage of OLEs (13\%) assigned the highest difference rating compared to RIs $(2.7 \%)$. 


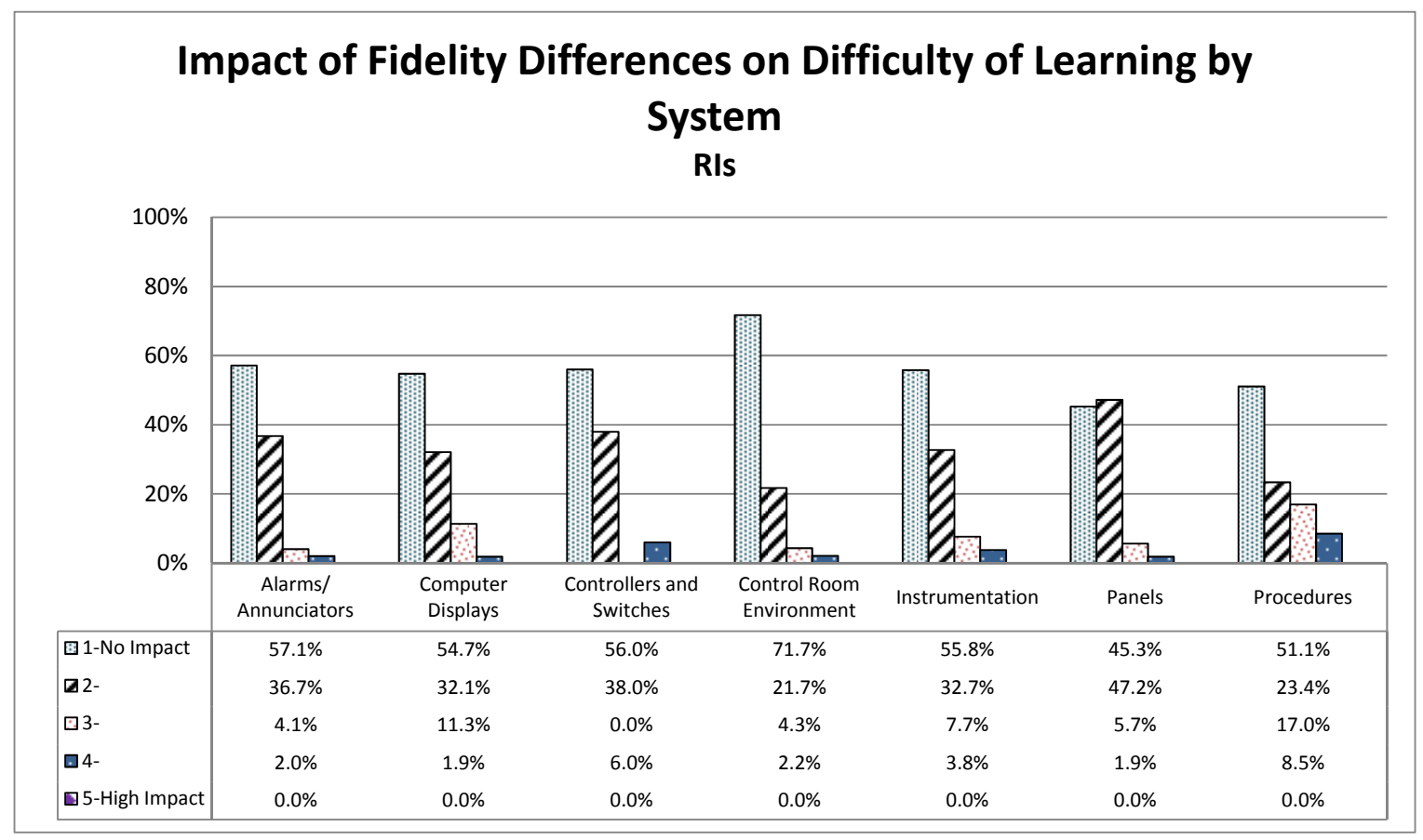

Figure 7.6. Fidelity Difference Impacts on Difficulty of Learning by System - RIs

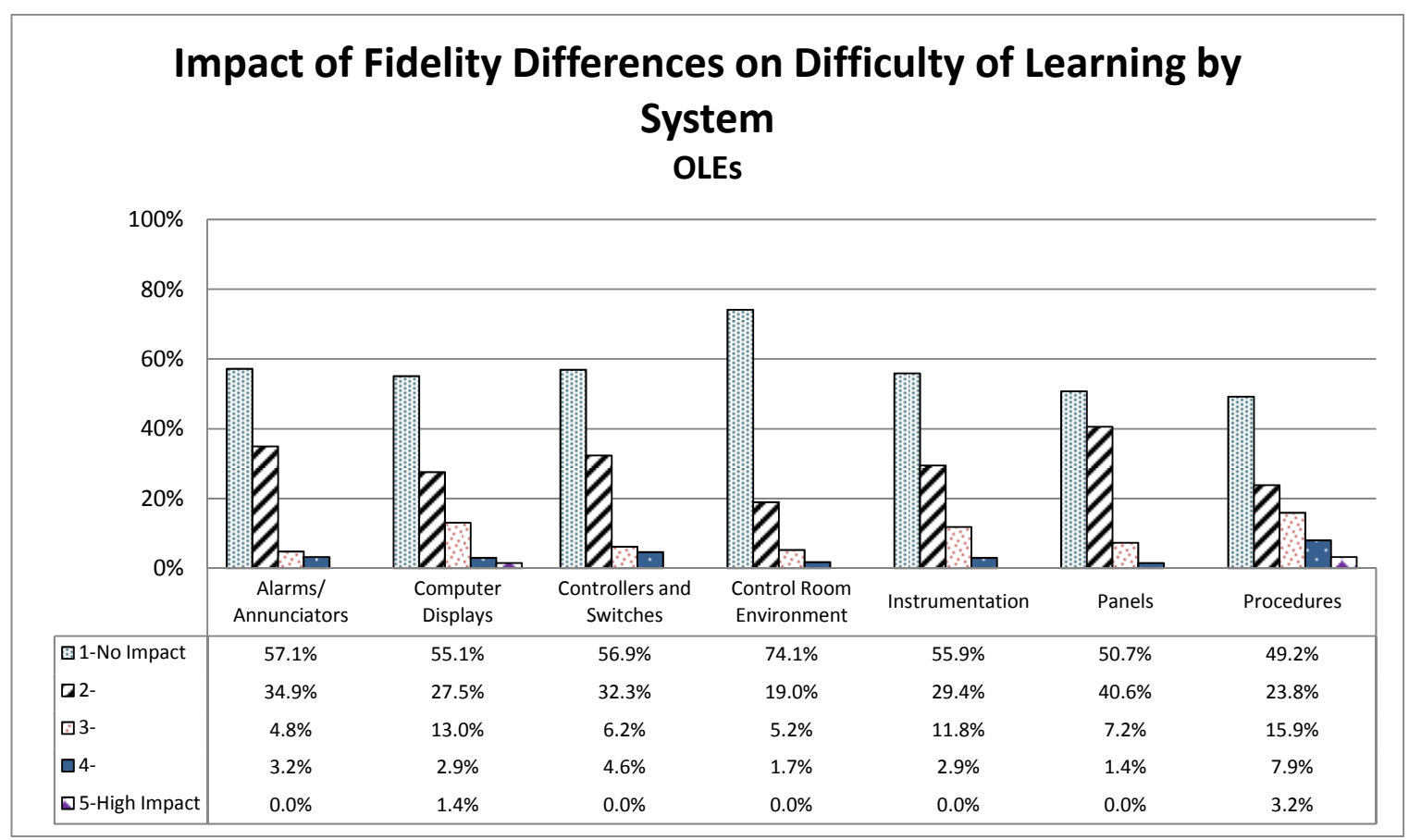

Figure 7.7. Fidelity Difference Impacts on Difficulty of Learning by System - OLEs

Average ratings of the impact on job performance from physical fidelity differences in alarms/ annunciators were 1.7 from RIs and 1.9 from OLEs with similar response patterns, although OLEs 
reported a slightly higher impact. Both RIs and OLEs rated the impact of physical fidelity differences in alarms/annunciators on difficulty of learning, low, with average group scores of 1.5 and 1.6, respectively, and similar response patterns.

\subsubsection{Computer Displays}

The average rating of fidelity difference by RIs and OLEs of computer displays was 3.5 and 3.6, respectively. The largest proportion of both groups rated computer display fidelity differences as 4 (32.4\% and $43.5 \%$, respectively), with $18.9 \%$ and $13 \%$ rating the difference as 5 .

The higher physical fidelity difference scores did not result in higher ratings of impact on job performance. RI and OLE respondents reported average impact scores of 1.9 and 2.0, respectively. With the exception of the 5 ratings by OLEs (4.3\%), the distribution of responses was similar for RIs and OLEs. The differences in computer display physical fidelity also did not result in high ratings of impact on difficulty of learning during refresher training. The average rating by RI respondents was 1.6, by OLE respondents it was 1.9. RIs overall found learning less affected by display differences: $32.1 \%$ of RIs rated the impact as 2 , and none rated the impact as 5 , while $6.3 \%$ of OLE respondents rated the impact on difficulty of learning as 5 .

\subsubsection{Controllers/Switches}

The average fidelity difference ratings of controllers by RI and OLE respondents were 3.1 and 2.8, respectively. Over half of OLEs and 37\% of RIs rated the difference a 3, with $73 \%$ of RIs rating the difference as 3 or above. RIs and OLEs exhibited similar patterns for 1 and 2 ratings, but a higher percentage of RIs than OLEs rated the differences as 4 and 5.

Very few respondents (5.8\% RI and 9.1\% OLE with ratings of 4 ) indicated that the impact from differences in controller/switches physical fidelity on job performance was high. The average impact rating was 2.0. The impact on training performance was also rated as low, with $60 \%$ and $56 \%$ of RIs and OLEs, respectively, rating the impact a 1, yielding an overall average score of 1.6.

\subsubsection{Control Room Environment}

OLEs rated the physical fidelity of the TTC CR environment with the CR environment at their assigned location higher (average score of 2.4) than RIs (average score of 3.0). While RIs scores were primarily distributed between 2 and 4, with approximately $25 \%$ in each, OLE scores were concentrated on 2 (43.5\%), although $21.7 \%$ of OLEs reported a high degree of fidelity, choosing 1 . Few OLEs (4.3\%) rated the CR environment of the TTC simulator "entirely different." The rating distribution of RIs was much different; $12.2 \%$ of RIs rated the difference in CR environment a 1 and $13.5 \%$ rated the difference as 5 .

Respondents reported that the fidelity differences in CR environment did not impact job performance. Average scores for RIs and OLEs for impact were 1.4 and 1.5, respectively, with $67.2 \%$ of RIs and $72.2 \%$ of OLEs reporting "no impact." The average scores for the impact of CR environment fidelity differences on learning difficulty were even lower, 1.4 for RIs and 1.3 for OLEs. A large majority of both RIs and 
OLEs rated the impact as 1 ( $71.7 \%$ and $83.3 \%$, respectively). However, a much larger proportion of RIs rated the impact a $2(21.7 \%)$ than OLEs $(8.3 \%)$.

\subsubsection{Instrumentation}

The average rating for differences in the physical fidelity of instrumentation for OLEs and RIs was 3.0 for RIs and 2.7 for OLEs. RIs reported greater instrumentation differences than OLEs; $24.3 \%$ of RIs responded "3," 36.5\% responded "4," and 4.1\% responded "5." Slightly over half (52.2\%) of OLEs responded that the instrumentation was somewhat different (a rating of 3), and 13\% rated the difference as 4 .

Despite these noticeable differences in the reported fidelity of instrumentation, both RIs and OLEs reported low average impacts on job performance, 2.0 and 1.9, respectively. The distribution of the two groups' scores was also similar. The reported impact of instrumentation fidelity differences on difficulty of learning was also low, averaging 1.6 for RIs and 1.7 for OLEs.

\subsubsection{Panel}

The average rating of physical fidelity differences of panels by RIs and OLEs was about 3.5, with over half responding with a rating of 4 or 5 . None of the respondents report that the TTC simulators' panels were "no different" than those at their assigned site and only about $14 \%$ of all respondents rated the difference a 2. The patterns of OLE and RI responses concerning the physical fidelity differences of TTC simulator panels are similar.

The reported impact of the differences in panel physical fidelity on job performance was fairly low for both OLEs and RIs - the overall average is 2.1, with about two-thirds of the respondents answering 1 or 2. The effect of panel differences on the difficulty of learning during refresher training was also rated low by both RIs and OLEs - the overall average was 1.6, with about $90 \%$ rating the impact a 1 or 2 .

\subsubsection{Procedural}

Average ratings of the difference in physical fidelity of procedures were 3.1 for RIs and 3.3 for OLEs, respectively. The rating patterns were similar for both groups, although the percentage of RIs rating the difference as 5 was slightly higher than OLEs (16.2\% for RIs and 13\% for OLEs).

RIs reported low impact on job performance from the difference in physical fidelity of procedures: $69.2 \%$ rated the impact as 1 or 2, and only $7.7 \%$ rated the impact as 4 or 5 . Many OLEs also rated the impact on job performance from difference in physical fidelity of procedures: $71.4 \%$ rated the impact as 1 or 2 , but $14.3 \%$ rated the impact as 4 or 5 . Impacts of fidelity differences in procedures on difficulty of learning were rated similarly. Both RIs and OLEs generally ( $74.5 \%$ and $73.0 \%$, respectively) rated the impact a 1 or 2 . 


\subsection{Summary of On-Line Survey Volunteered Comments about Physical Fidelity Differences and Impacts}

Question: Please describe the differences in PANELS that had the most significant effect on your ability to perform you job duties after your initial simulator training or learn course lessons during refresher training.

Comment Summary: In general, OLE personnel were not much affected by differences in panels between the TTC simulators and the simulators at actual plants. They did not expect them to be identical. Differences in safety related panels and the panels controlling ECCS or reactivity were more important to OLE personnel than differences in panels controlling auxiliary equipment. RI personnel did not expect the TTC simulator panels to be identical to the simulator and CR of their assigned plants, and used the TTC simulator only for overall familiarization. Differences in safety-related panels and the panels controlling ECCS or reactivity were more important to RI personnel than differences in panels controlling auxiliary equipment.

Question: Please describe the differences in CONTROLLERS/SWITCHES that had the most significant effect on your ability to perform your job duties after your initial simulator training or learn course lessons during refresher training.

Comment Summary: OLE personnel found the TTC simulators close enough to the plants they will give exams at for familiarization. They stated that extreme fidelity would not be valuable as they are not operators. They noted that some plants have installed digital controllers that are quite different from the analog TTC controllers. The exact type of controller or switch is less important than familiarization with how they work in general. RI personnel stressed that they don't operate switches/controllers so extreme fidelity was not necessary. An upgrade of TTC simulators to include at least some digital controllers would be helpful (11 out of total 83 respondents made this comment).

Question: Please describe the differences in INSTRUMENTATION that had the most significant effect on your ability to perform your job duties after your initial simulator training or learn course lessons during refresher training.

Comment Summary: OLE personnel found the TTC simulators close enough for the most part, but noted that the plants are going more to digital instrumentation and recorders, while TTC has strip charts (18 out of total 83 respondents commented on the plants having more digital controllers than TTC).

Question: Please describe the differences in ALARMS/ANNUNCIATORS that had the most significant effect on your ability to perform your job duties after your initial simulator training or learn course lessons during refresher training.

Comment Summary: OLE personnel found the TTC simulators close enough to the plants they will give exams at for familiarization, and felt that extreme fidelity would not be valuable as they are not operators. This is particularly true of alarms and annunciators where the differences 
between TTC and the plants are generally not important to the OLE or RI personnel. One RI person indicated that the TTC alarm response procedures could be improved.

Question: Please describe the differences in COMPUTER DISPLAYS that had the most significant effect on your ability to perform your job duties after your initial simulator training or learn course lessons during refresher training.

Comment Summary: OLE personnel found the TTC simulators close enough to the plants at which they will give exams for familiarization and felt that extreme fidelity would not be valuable as they are not operators. The operating plants generally have more modern computer displays, but the TTC displays are close enough for training. RI personnel mostly said they don't use the plant computer very much so the differences were not important.

Question: Please describe the differences in PROCEDURES that had the most significant effect on your ability to perform your job duties after your initial simulator training or learn course lessons during refresher training. Please do not consider differences in Procedure content.

Comment Summary: OLE personnel found the TTC simulators' procedures to be out of date, not very similar to the actual EOI, AOI, and alarm response procedures, and recommended that they should be made more like operating plant procedures. TTC procedures omit important nonsafety systems like instrument air and circulation water. RI personnel also had problems with differences in procedures between TTC and their plants, with the TTC procedures being old, obsolete, and/or lacking detail. Some RI personnel said the TTC procedures were close enough for training, some said they were not.

Question: Please describe the differences in CONTROL ROOM ENVIRONMENT that had the most significant effect on your ability to perform your job duties after your initial simulator training or learn course lessons during refresher training:

Comment Summary: OLE personnel found the TTC simulators had a different ambiance, with no typical ventilation noise, administration support area, etc. Some OLE personnel thought this was important and some did not. Some RI personnel noted that the TTC Westinghouse simulator was more realistic than the others. The typical formal access requirements to the CR of a real plant are not generally duplicated at TTC, which may mislead new personnel about how and when to access the CR. The "at the controls" area is marked on the floor of some plants, but not at TTC, which was considered to be an easy fix.

Question: [License Examiner] Once at the site-specific simulator what did you have to do, if anything, to deal with the [physical fidelity] differences [between the TTC simulator on which you were trained and the site-specific simulator]? OR [Resident] Once at your assigned CR what did you have to do, if anything, to deal with the [physical fidelity] differences [between the TTC simulator on which you were trained and the CR at the plant to which you are currently assigned]?

Comment Summary: OLE personnel found the TTC simulators close enough to the plants at which they will give exams for familiarization and felt that a quick walkdown for familiarization at the actual plant was sufficient to deal with differences. 
Question: Please add any comments you have on the physical fidelity of simulators for training.

Comment Summary: Five respondents suggested that any new simulator needed to have exact fidelity, one pointing out that the new (Gen III+) designs would have standardized CRs that could be duplicated at TTC. Seventeen opined that exact fidelity was not important.

\subsection{How TTC Training Addressed Physical Fidelity Differences}

OLEs and RIs were asked to report the extent to which fidelity differences presented by the simulator in their training were addressed. The survey asked respondents to rate separately the degree to which differences in fidelity were addressed and the contribution each of the following resources made in addressing fidelity differences:

- instructors

- training materials

- student discussions.

The responses are presented in Figure 7.8, which illustrates the differences in responses between RIs and OLEs on these questions. As shown in this figure, $48.6 \%$ of RIs and $63.6 \%$ of OLEs $(52 \%$ of total responses) reported that instructors explained differences in some detail. While the total average score of 2.4 suggests that more detail was left out than included, $64 \%$ of OLEs and $48.6 \%$ of RIs reported that instructors explained differences in great detail. However, $27 \%$ of OLEs reported that differences were neither explained nor pointed out, compared to $18 \%$ of RIs. Twenty-eight percent of RIs reported that differences were pointed out, but without detail.

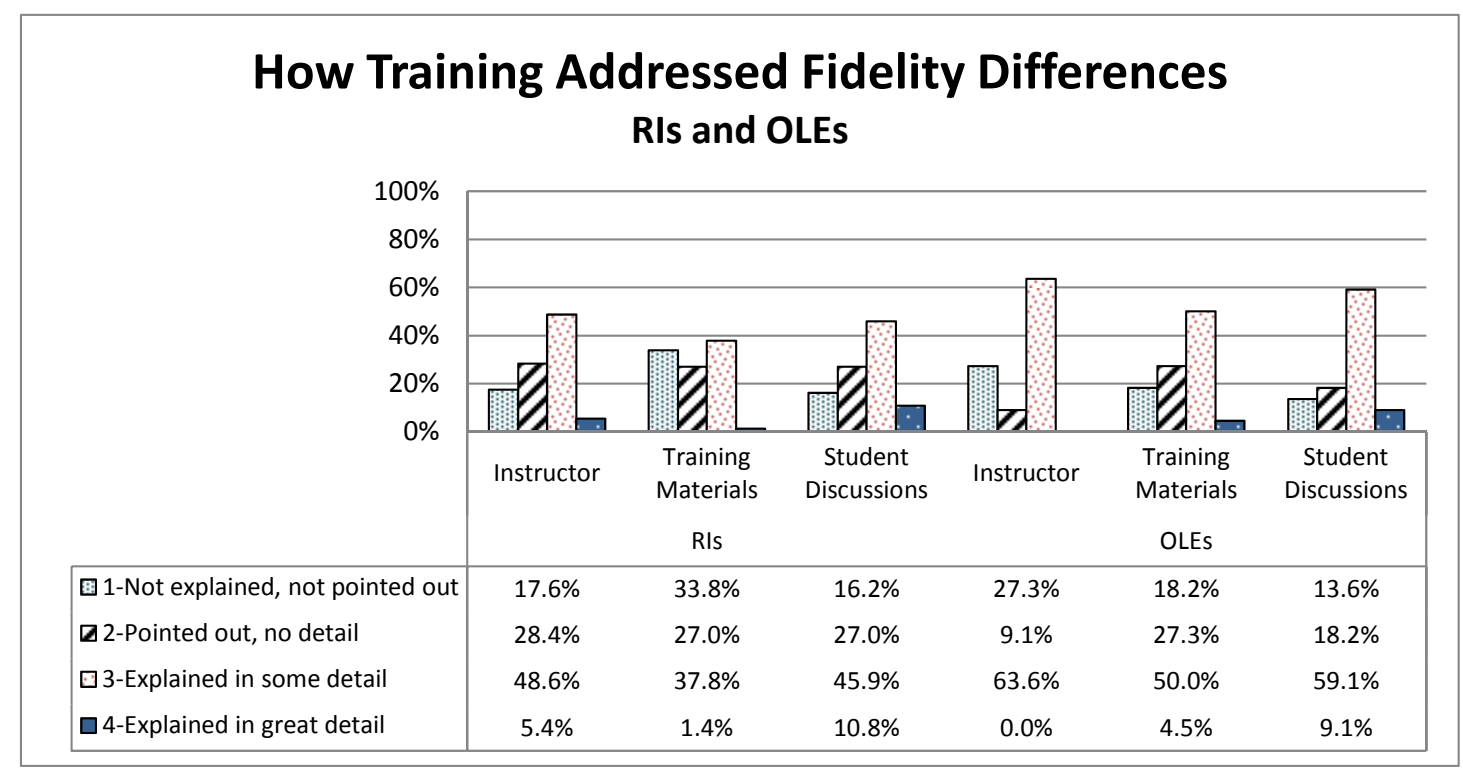

Figure 7.8. How Training Addressed Fidelity Differences - RIs and OLEs

With regard to the extent to which training materials pointed out and explained differences in fidelity, Figure 7.8 shows that, for RIs, approximately similar percentages of respondents reported that the 
materials neither explained nor pointed out; pointed out, but with no detail; and explained in some detail the fidelity differences. This pattern was quite different for OLEs, who more frequently indicated that the training materials explained the fidelity differences in some detail, and with less frequency indicated that fidelity differences were neither explained nor pointed out. Few RIs or OLEs reported that the training materials explained the fidelity differences in great detail.

RIs and OLEs responding to the survey showed a similar pattern in their reports of the extent to which student discussions addressed and explained fidelity differences. For both groups, the most frequent response was that student discussions explained fidelity differences in some detail (45.9\% for RIs, 59.1\% for OLEs). Fewer respondents rated student discussions as neither explaining nor pointing out fidelity differences than either instructors or materials (16.2\% for RIs and $13.6 \%$ for OLEs). More RIs and OLEs indicated that student discussions explained fidelity differences in great detail $(10.8 \%$ and $9.1 \%$, respectively) compared to either instructors or training materials.

Generally simulator differences are being pointed out or explained, with $91 \%$ of all the respondents indicating that the differences were at least pointed out, with or without detail, by one or more of the three resources. Moreover, $72 \%$ of all the respondents received an explanation of the differences with at least some detail by one or more of the three resources. It may not be surprising that other students are the best resources, given their combined experience in a variety of CRs, the number of students available relative to instructors, and the difficulties in creating training materials addressing fidelity differences given the high degree of variance in CR HSI systems among currently operating reactors.

In the free-text comments provided on the survey, many respondents mentioned self-study, OJT, or simply "adapting" as ways to address differences. Respondents reported using resources at their site in the form of other NRC personnel assigned to the same site, simulator time at the plant, CR walkdowns, and attending operator training for the assigned site to learn about fidelity differences. These resources rely on the trainee to identify the differences with the TTC simulator.

No relationship was shown between the level of fidelity differences reported by the respondents and reports of receiving information on the fidelity differences during training. Similarly, no relationship was shown between the level of fidelity differences reported by the respondents and the level of explanatory detail given on the fidelity differences.

For particular HSI systems, a moderate relationship was shown between the level of detail provided by instructors on fidelity differences and the impact these differences had on how difficult it was for the trainees to learn course lessons during the refresher training. When instructors gave more detail in their explanation of fidelity differences, fidelity differences in Procedures $(\mathrm{r}=0.36)$, Alarms/Annunciators $(\mathrm{r}=0.29)$, and Instrumentation $(\mathrm{r}=0.22)$ had less of an impact on their difficulty of learning in refresher courses.

\subsection{Implications for Physical Fidelity of TTC Simulators in Advanced Reactor Training}

All current NRC RIs and OLEs were asked to complete an on-line survey that focused on physical fidelity differences between existing TTC training simulators and the reactor plants where the respondents work, and the significance of those differences to TTC trainees. Seventy-four RIs and 23 OLEs 
completed the survey. In general the respondents, both RIs and OLEs, considered the differences in physical fidelity to be moderate, but the impact of these differences on their job performance and difficulty of learning to be low. OLEs generally rated the impact of fidelity differences on job performance to be higher than RIs did, with differences in procedures, panels, and computer displays identified as causing the greatest impact $(14.3 \%, 13 \%$, and $13 \%$, of OLEs rating impacts at 4 or 5 out of 5 , respectively). The respondents generally expressed the opinion that TTC training simulators needed to have enough fidelity to teach concepts and the general layout of nuclear plant CRs, but that greater physical fidelity was not needed. They reported that the current TTC simulators generally do provide this sufficient level of fidelity, but suggested some updates that might be useful, such as adding an "at the controls" area on the TTC simulator floor, providing at least a few modern digital type controllers (that many current operating plants have installed as upgrades or replacements of their original analog controllers), and updating procedures to be more representative of operating plants. Many respondents commented that because NRC staff members are not operators, and the simulator training course is relatively brief, a high level of physical fidelity is not needed for effective training. 


\subsection{Conclusions}

\subsection{Summary of Factors Influencing Simulator Physical Fidelity Training Priorities and Needs: Current and Future}

Based on the analyses conducted for this study, the professional judgment of the team is that the Task/KSA Inventory developed for the current RI and OLE positions and itemized in Section 5.1 will remain relevant to NRC regulatory personnel working on Gen III/Gen III+ reactors because these advanced reactors represent evolutionary developments of current reactors, with similar safety and security systems, and they will be subject to essentially the same regulations as current plants. Consequently, the team has concluded that the training needs analysis conducted in this study provides information pertinent to both the training needs for staff responsible for regulating and overseeing these advanced reactors and to physical fidelity considerations for TTC training simulators.

The project team has also concluded that the RI and OLE Task/KSA Inventory can serve as a foundation for evaluating the physical fidelity needs of TTC advanced reactor training simulators by examining each inventory item in terms of the following dimensions:

- What is the training priority of the Task/KSA Inventory item (regardless of training technology characteristics)? [Source: DIF analysis, Section 5.2]

- How important, or likely to be helpful, is a simulator for training the Task/KSA item? [Source: expert panel rating, Section 5.3]

- How difficult is the Task/KSA item to learn? [Source: expert panel rating, Section 5.3]

- To what extent does the Task/KSA item require integration of information? [Source: team judgment based on discussions with TTC staff and students]

- What types and levels of knowledge or skill are needed for this item? [Source: expert panel rating, Section 5.4]

- How important are each of the HSI systems to this Task/KSA? [Source: expert panel rating, Section 5.5]

- How much impact do differences in physical fidelity for the important HSI systems have on job performance? [Source: online survey, Section 7.2]

- What level of fidelity is likely to be needed, and is the physical fidelity of any particular HSI systems likely to be especially important? [integrated analysis]

The project team combined the following items in order to provide a basis for focusing the assessment of simulator physical fidelity needs on items most important to training and for which simulator fidelity is likely to be most important:

- task/KSA Inventory, which identifies at a relatively high level of accuracy that which the RIs and OLEs must learn during training

- DIF analysis, which identifies training priorities among the Task/KSA items 
- ratings of difficulty to learn, importance of using a simulator to learn, and level of integrated and procedural knowledge, visual and auditory skill, and facility in accessing external information needed for each inventory item

These factors reflect findings from the literature and the information gained from the expert panels and NRC staff. Although there can be, and probably are, additional factors that are important to considerations of physical fidelity training needs, the project team determined that these represented a useful framework for considering which of the Task/KSA Inventory items, and hence which HSI systems should receive the most attention when considering physical fidelity needs. Table 8.1 presents this rating information for each item in the Task/KSA Inventory.

The last column in Table 8.2 is a simple sum of the eight factors. The Task/KSA Inventory items with high training priority scores ( 7 or higher) that are not among the top composite score items (largely $[\mathrm{K}]$ items that were not scored for five of the factors $)^{1}$ are included in the items identified as warranting particular examination for simulator fidelity implications. Although it can be argued that weighting factors should be applied to at least some of these factors, a clear basis for assigning weighting was not evident. Therefore, because this subset of items is used only to focus subsequent analysis, the team determined that this straightforward method was adequate.

Table 8.3 presents the subset of Task/KSA items with high composite scores across these eight factors, along with the remaining items with DIF scores of 7 or higher. Table 8.4 is included to aid the reader by presenting the text of the top rated Task/KSA Inventory Items shown in Table 8.2.

Five of the six Task/KSA Inventory items with highest composite score are operations tasks, and all involve evaluations of operator performance. The top four of these are RI tasks, while the fifth is the OLE overall task of evaluating emergency procedure use by the crew during an examination. Interestingly, the Task/KSA Inventory item with the highest DIF score and a tie for highest composite score, Ops-16-RI (Gather information and communicate with the NRC during declared emergencies) was rated only 3 out of 5 for the importance of using a simulator for learning. In contrast to this, all of these top six items had high scores in the remaining factors we considered to indicate importance for training and potentially influential in determining the need for simulator physical fidelity. Each of the top 12 items by composite score is an (Ops) item. Because items in the Task/KSA Inventory were not all rated on each of the eight dimensions, Tables 8.1 and 8.2 include the remaining items with the highest DIF scores $(7$ or above). Many of these items are the (K) items that represent the knowledge about the plant against which the remainder of the Task/KSA Inventory items were rated.

Table 8.4 presents the ratings of HSI system importance to this subset of Task/KSA Inventory items. Combining this information with the ratings of impact on job performance from differences in the physical fidelity of these HSI systems, presented in Figures 7.4 and 7.5 in Section 7.2, brings together information that helps inform the analysis of physical fidelity needs and consequences. Although this information is not sufficient to specify physical fidelity parameters for these simulators, the project team agrees that it provides a basis for focusing and framing the investigation of fidelity needs.

\footnotetext{
${ }^{1}$ See Section 3.4 for discussion of the treatment of these items in the rating of type and level of knowledge, skill, and ability needed for each item. The team realized that five of the Task/KSA Inventory items (K-1, K-2, K-3, K-6, and K-7) represented knowledge about the plant rather than about operator CR behavior, and were therefore better addressed as a type of knowledge against which the remainder of the Task/KSA Inventory items should be rated.
} 
Table 8.1. Ratings on Factors Influencing Fidelity Needs for Training Task/KSA Inventory Items

\begin{tabular}{|c|c|c|c|c|c|c|c|c|c|c|}
\hline & & $\begin{array}{l}\text { Training } \\
\text { Priority (DIF) } \\
(1=\text { low to } \\
10=\text { high) } \\
\end{array}$ & $\begin{array}{l}\text { Importance of } \\
\text { Using a Simulator } \\
\text { for Learning to } \\
\text { Competently } \\
\text { Perform Task } \\
(1=\text { low to } \\
5=\text { high }) \\
\end{array}$ & $\begin{array}{l}\text { Difficulty of } \\
\text { Learning } \\
(1=\text { low to } \\
5=\text { high }) \\
\end{array}$ & $\begin{array}{c}\text { Requires } \\
\text { Integrated } \\
\text { Knowledge? } \\
(\sqrt{ }=\text { yes }) \\
\end{array}$ & $\begin{array}{c}\text { Requires } \\
\text { Visual Skill } \\
\text { at the Level } \\
\text { of Familiarity } \\
\text { ( } \sqrt{ }=\text { yes) }\end{array}$ & $\begin{array}{l}\text { Requires } \\
\text { Auditory Skill } \\
\text { at the Level of } \\
\text { Familiarity } \\
(\sqrt{ }=\text { yes })\end{array}$ & $\begin{array}{c}\text { Procedural Knowledge } \\
\text { Level Required ( } 2= \\
\text { need to know purpose; } \\
3=\text { need to know how it } \\
\text { is done but not how to } \\
\text { do it; } 4=\text { need to know } \\
\text { how to execute, but not } \\
\text { with skill/speed) }\end{array}$ & $\begin{array}{l}\text { Requires Quick } \\
\text { Access to } \\
\text { External } \\
\text { Information } \\
\text { (implies higher } \\
\text { level of physical } \\
\text { fidelity is } \\
\text { needed) }(\sqrt{ }=\text { yes) } \\
\end{array}$ & $\begin{array}{c}\text { Composite } \\
\text { Score (checks } \\
\text { counted as } \\
\text { "1") }\end{array}$ \\
\hline (Ops-1) & (RI) & 5 & 4 & 3 & $\sqrt{ }$ & $\sqrt{ }$ & $\sqrt{ }$ & 3 & & 18 \\
\hline (Ops-1) & (OLE) & 6 & 5 & 4 & $\sqrt{ }$ & $\sqrt{ }$ & $\sqrt{ }$ & 3 & & 21 \\
\hline (Ops-2) & (RI) & 5 & 4 & 4 & $\sqrt{ }$ & $\sqrt{ }$ & $\sqrt{ }$ & 3 & $\sqrt{ }$ & 20 \\
\hline (Ops-2) & (OLE) & 4 & 5 & 3 & $\sqrt{ }$ & $\sqrt{ }$ & $\sqrt{ }$ & 3 & $\sqrt{ }$ & 19 \\
\hline (Ops-3) & (RI) & 8 & 5 & 4 & $\sqrt{ }$ & $\sqrt{ }$ & $\sqrt{ }$ & 3 & $\sqrt{ }$ & 24 \\
\hline (Ops-3) & (OLE) & 5 & 5 & 3 & $\sqrt{ }$ & $\sqrt{ }$ & $\sqrt{ }$ & 3 & $\sqrt{ }$ & 20 \\
\hline (Ops-4) & (RI)a & 7 & 4 & 4 & $\sqrt{ }$ & $\sqrt{ }$ & $\sqrt{ }$ & 4 & $\sqrt{ }$ & 23 \\
\hline (Ops-4) & $(\mathrm{RI}) \mathrm{b}$ & 6 & 4 & 4 & $\sqrt{ }$ & $\sqrt{ }$ & $\sqrt{ }$ & 3 & & 20 \\
\hline (Ops-4) & (OLE) & 5 & 3 & 4 & $\sqrt{ }$ & $\sqrt{ }$ & $\sqrt{ }$ & 3 & & 18 \\
\hline (Ops-5) & (RI) & 7 & 4 & 4 & $\sqrt{ }$ & $\sqrt{ }$ & $\sqrt{ }$ & 3 & $\sqrt{ }$ & 22 \\
\hline (Ops-5) & (OLE) & 7 & 4 & 4 & $\sqrt{ }$ & $\sqrt{ }$ & $\sqrt{ }$ & 3 & $\sqrt{ }$ & 22 \\
\hline (Ops-6) & (RI) & 6 & 4 & 4 & $\sqrt{ }$ & $\sqrt{ }$ & $\sqrt{ }$ & 4 & $\sqrt{ }$ & 22 \\
\hline (Ops-6) & (OLE) & 6 & 5 & 3 & $\sqrt{ }$ & $\sqrt{ }$ & $\sqrt{ }$ & 3 & $\sqrt{ }$ & 21 \\
\hline (Ops-7) & (RI) & 5 & 4 & 3 & $\sqrt{ }$ & $\sqrt{ }$ & $\sqrt{ }$ & 4 & & 19 \\
\hline (Ops-7) & (OLE) & 4 & 5 & 3 & $\sqrt{ }$ & $\sqrt{ }$ & $\sqrt{ }$ & 4 & & 19 \\
\hline (Ops-8) & (RI) & 6 & 4 & 3 & $\sqrt{ }$ & $\sqrt{ }$ & $\sqrt{ }$ & 3 & $\sqrt{ }$ & 20 \\
\hline (Ops-8) & (OLE) & 5 & 5 & 3 & $\sqrt{ }$ & $\sqrt{ }$ & $\sqrt{ }$ & 3 & $\sqrt{ }$ & 20 \\
\hline (Ops-9) & (RI) & 8 & 5 & 4 & $\sqrt{ }$ & $\sqrt{ }$ & $\sqrt{ }$ & 4 & $\sqrt{ }$ & 25 \\
\hline (Ops-9) & (OLE) & 7 & 5 & 4 & $\sqrt{ }$ & $\sqrt{ }$ & $\sqrt{ }$ & 4 & $\sqrt{ }$ & 24 \\
\hline (Ops-10) & (RI) & 8 & 5 & 4 & $\sqrt{ }$ & $\sqrt{ }$ & $\sqrt{ }$ & 4 & & 24 \\
\hline (Ops-10) & (OLE) & 6 & 3 & 3 & $\sqrt{ }$ & $\sqrt{ }$ & $\sqrt{ }$ & 4 & & 19 \\
\hline (Ops-11) & (RI) & 6 & 3 & 4 & $\sqrt{ }$ & $\sqrt{ }$ & $\sqrt{ }$ & 3 & & 19 \\
\hline (Ops-11) & (OLE) & 5 & 1 & 2 & $\sqrt{ }$ & $\sqrt{ }$ & $\sqrt{ }$ & 3 & & 14 \\
\hline (Ops-12) & (RI) & 6 & 4 & 3 & $\sqrt{ }$ & $\sqrt{ }$ & $\sqrt{ }$ & 3 & $\sqrt{ }$ & 20 \\
\hline (Ops-12) & (OLE) & 4 & 5 & 2 & $\sqrt{ }$ & $\sqrt{ }$ & $\sqrt{ }$ & 3 & $\sqrt{ }$ & 18 \\
\hline (Ops-13) & (RI) & 8 & 3 & 3 & $\sqrt{ }$ & $\sqrt{ }$ & $\sqrt{ }$ & 3 & $\sqrt{ }$ & 21 \\
\hline (Ops-13) & (OLE) & 5 & 3 & 3 & $\sqrt{ }$ & $\sqrt{ }$ & $\sqrt{ }$ & 3 & $\sqrt{ }$ & 18 \\
\hline
\end{tabular}


Table 8.1. (contd)

\begin{tabular}{|c|c|c|c|c|c|c|c|c|c|c|}
\hline & & $\begin{array}{c}\text { Training } \\
\text { Priority (DIF) } \\
(1=\text { low to } \\
10=\text { high) }\end{array}$ & $\begin{array}{l}\text { Importance of } \\
\text { Using a Simulator } \\
\text { for Learning to } \\
\text { Competently } \\
\text { Perform Task } \\
(1=\text { low to } \\
5=\text { high }) \\
\end{array}$ & $\begin{array}{c}\text { Difficulty of } \\
\text { Learning } \\
(1=\text { low to } \\
5=\text { high }) \\
\end{array}$ & $\begin{array}{c}\text { Requires } \\
\text { Integrated } \\
\text { Knowledge? } \\
(\sqrt{ }=\text { yes }) \\
\end{array}$ & $\begin{array}{c}\text { Requires } \\
\text { Visual Skill } \\
\text { at the Level } \\
\text { of Familiarity } \\
(\sqrt{ }=\text { yes })\end{array}$ & $\begin{array}{c}\text { Requires } \\
\text { Auditory Skill } \\
\text { at the Level of } \\
\text { Familiarity } \\
(\sqrt{ }=\text { yes })\end{array}$ & $\begin{array}{c}\text { Procedural Knowledge } \\
\text { Level Required ( } 2= \\
\text { need to know purpose; } \\
3=\text { need to know how it } \\
\text { is done but not how to } \\
\text { do it; } 4=\text { need to know } \\
\text { how to execute, but not } \\
\text { with skill/speed) }\end{array}$ & $\begin{array}{c}\text { Requires Quick } \\
\text { Access to } \\
\text { External } \\
\text { Information } \\
\text { (implies higher } \\
\text { level of physical } \\
\text { fidelity is } \\
\text { needed) }(\sqrt{ }=\text { yes) } \\
\end{array}$ & $\begin{array}{c}\text { Composite } \\
\text { Score (checks } \\
\text { counted as } \\
\text { "1") }\end{array}$ \\
\hline (Ops-14) & (RI) & 3 & 3 & 3 & $\sqrt{ }$ & $\sqrt{ }$ & $\sqrt{ }$ & 3 & & 15 \\
\hline (Ops-14) & (OLE) & 4 & 3 & 3 & $\sqrt{ }$ & $\sqrt{ }$ & $\sqrt{ }$ & 3 & & 16 \\
\hline (Ops-15) & (RI) & 2 & 2 & 3 & $\sqrt{ }$ & & & 3 & & 11 \\
\hline (Ops-15) & (OLE) & 4 & 1 & 3 & $\sqrt{ }$ & & & 3 & & 12 \\
\hline (Ops-16) & (RI) & 10 & 3 & 4 & $\sqrt{ }$ & $\sqrt{ }$ & $\sqrt{ }$ & 4 & $\sqrt{ }$ & 25 \\
\hline (Ops-16) & (OLE) & 6 & 1 & 2 & $\sqrt{ }$ & $\sqrt{ }$ & $\sqrt{ }$ & 4 & $\sqrt{ }$ & 17 \\
\hline (Mgmt-1) & (RI) & 6 & 4 & 3 & & $\sqrt{ }$ & $\sqrt{ }$ & 2 & & 17 \\
\hline (Mgmt-1) & (OLE) & 6 & 5 & 4 & & $\sqrt{ }$ & $\sqrt{ }$ & 2 & & 19 \\
\hline (Mgmt-2) & (RI) & 6 & 2 & 3 & $\sqrt{ }$ & $\sqrt{ }$ & $\sqrt{ }$ & 3 & & 17 \\
\hline (Mgmt-2) & (OLE) & 5 & 2 & 2 & $\sqrt{ }$ & $\sqrt{ }$ & $\sqrt{ }$ & 3 & & 15 \\
\hline (Mgmt-3) & (RI) & 5 & 2 & 3 & $\sqrt{ }$ & & & 3 & & 15 \\
\hline (Mgmt-3) & (OLE) & 2 & 1 & 2 & $\sqrt{ }$ & & & 3 & & 10 \\
\hline (Mgmt-4) & $(\mathrm{RI})$ & 5 & 2 & 3 & $\sqrt{ }$ & $\sqrt{ }$ & & 3 & & 15 \\
\hline (Mgmt-4) & (OLE) & 1 & 1 & 2 & $\sqrt{ }$ & $\sqrt{ }$ & & 3 & & 9 \\
\hline (Mgmt-5) & (RI) & 5 & 3 & 4 & $\sqrt{ }$ & $\sqrt{ }$ & & 4 & & 18 \\
\hline (Mgmt-5) & (OLE) & 5 & 1 & 4 & $\sqrt{ }$ & $\sqrt{ }$ & & 4 & & 16 \\
\hline (Lic-1) & (RI) & 6 & 3 & 4 & $\sqrt{ }$ & $\sqrt{ }$ & $\sqrt{ }$ & 3 & & 19 \\
\hline (Lic-1) & (OLE) & 5 & 1 & 4 & $\sqrt{ }$ & $\sqrt{ }$ & $\sqrt{ }$ & 3 & & 16 \\
\hline (Lic-2) & (RI) & 7 & 3 & 3 & $\sqrt{ }$ & $\sqrt{ }$ & $\sqrt{ }$ & 3 & & 19 \\
\hline$($ Lic-2) & (OLE) & 5 & 2 & 3 & $\sqrt{ }$ & $\sqrt{ }$ & $\sqrt{ }$ & 3 & & 16 \\
\hline$(\mathrm{K}-1)$ & (RI) & 5 & 4 & 3 & N/A & N/A & N/A & N/A & N/A & 12 \\
\hline$(\mathrm{K}-1)$ & (OLE) & 7 & 3 & 5 & N/A & $\mathrm{N} / \mathrm{A}$ & N/A & N/A & $\mathrm{N} / \mathrm{A}$ & 15 \\
\hline$(\mathrm{K}-2)$ & (RI) & 7 & 4 & 4 & N/A & $\mathrm{N} / \mathrm{A}$ & N/A & N/A & N/A & 15 \\
\hline$(\mathrm{K}-2)$ & (OLE) & 8 & 4 & 5 & N/A & $\mathrm{N} / \mathrm{A}$ & N/A & $\mathrm{N} / \mathrm{A}$ & $\mathrm{N} / \mathrm{A}$ & 17 \\
\hline$(\mathrm{K}-3)$ & $(\mathrm{RI})$ & 6 & 3 & 4 & N/A & $\mathrm{N} / \mathrm{A}$ & N/A & $\mathrm{N} / \mathrm{A}$ & $\mathrm{N} / \mathrm{A}$ & 13 \\
\hline (K-3) & (OLE) & 8 & 4 & 5 & N/A & N/A & N/A & N/A & N/A & 17 \\
\hline (K-4) & (RI) & 7 & 3 & 4 & $\sqrt{ }$ & & & 3 & & 18 \\
\hline (K-4) & (OLE) & 7 & 3 & 4 & $\sqrt{ }$ & & & 3 & & 18 \\
\hline
\end{tabular}


Table 8.1. ( contd)

\begin{tabular}{|c|c|c|c|c|c|c|c|c|c|c|}
\hline & & $\begin{array}{c}\text { Training } \\
\text { Priority (DIF) } \\
(1=\text { low to } \\
10=\text { high) }\end{array}$ & $\begin{array}{l}\text { Importance of } \\
\text { Using a Simulator } \\
\text { for Learning to } \\
\text { Competently } \\
\text { Perform Task } \\
(1=\text { low to } \\
5=\text { high })\end{array}$ & $\begin{array}{l}\text { Difficulty of } \\
\text { Learning } \\
(1=\text { low to } \\
5=\text { high })\end{array}$ & $\begin{array}{c}\text { Requires } \\
\text { Integrated } \\
\text { Knowledge? } \\
(\sqrt{ }=\text { yes })\end{array}$ & $\begin{array}{c}\text { Requires } \\
\text { Visual Skill } \\
\text { at the Level } \\
\text { of Familiarity } \\
(\sqrt{ }=\text { yes })\end{array}$ & $\begin{array}{l}\text { Requires } \\
\text { Auditory Skill } \\
\text { at the Level of } \\
\text { Familiarity } \\
(\sqrt{ }=\text { yes })\end{array}$ & $\begin{array}{c}\text { Procedural Knowledge } \\
\text { Level Required }(2= \\
\text { need to know purpose; } \\
3=\text { need to know how it } \\
\text { is done but not how to } \\
\text { do it; } 4=\text { need to know } \\
\text { how to execute, but not } \\
\text { with skill/speed) }\end{array}$ & $\begin{array}{l}\text { Requires Quick } \\
\text { Access to } \\
\text { External } \\
\text { Information } \\
\text { (implies higher } \\
\text { level of physical } \\
\text { fidelity is } \\
\text { needed) }(\sqrt{ }=\text { yes) } \\
\end{array}$ & $\begin{array}{c}\text { Composite } \\
\text { Score (checks } \\
\text { counted as } \\
\text { "1") }\end{array}$ \\
\hline$(\mathrm{K}-5)$ & (RI) & 6 & 3 & 4 & $\sqrt{ }$ & & & 3 & & 17 \\
\hline$(\mathrm{K}-5)$ & (OLE) & 6 & 2 & 4 & $\sqrt{ }$ & & & 3 & & 16 \\
\hline$(\mathrm{K}-6)$ & $(\mathrm{RI})$ & 7 & 3 & 4 & N/A & $\mathrm{N} / \mathrm{A}$ & N/A & N/A & $\mathrm{N} / \mathrm{A}$ & 14 \\
\hline$(\mathrm{K}-6)$ & (OLE) & 8 & 4 & 5 & $\mathrm{~N} / \mathrm{A}$ & $\mathrm{N} / \mathrm{A}$ & N/A & $\mathrm{N} / \mathrm{A}$ & N/A & 17 \\
\hline$(\mathrm{K}-7)$ & (RI) & 7 & 3 & 4 & N/A & N/A & N/A & N/A & N/A & 14 \\
\hline (K-7) & (OLE) & 8 & 4 & 5 & N/A & N/A & N/A & N/A & N/A & 17 \\
\hline
\end{tabular}


Table 8.2. Top-Rated Task/KSA Inventory Items in Terms of Training Priority and Other Factors

\begin{tabular}{|c|c|c|c|c|c|c|c|c|c|c|}
\hline & & $\begin{array}{c}\text { DIF } \\
\text { Score }\end{array}$ & $\begin{array}{c}\text { Importance } \\
\text { of } \\
\text { Simulator }\end{array}$ & $\begin{array}{c}\text { Difficulty } \\
\text { of } \\
\text { Learning }\end{array}$ & $\begin{array}{l}\text { Requires } \\
\text { Integrated } \\
\text { Knowledge }\end{array}$ & $\begin{array}{c}\text { Requires } \\
\text { Visual } \\
\text { Skill }\end{array}$ & $\begin{array}{c}\text { Requires } \\
\text { Auditory } \\
\text { Skill }\end{array}$ & $\begin{array}{l}\text { Level of } \\
\text { Procedural } \\
\text { Knowledge }\end{array}$ & $\begin{array}{c}\text { Requires } \\
\text { Quick } \\
\text { Access to } \\
\text { External } \\
\text { Information }\end{array}$ & $\begin{array}{c}\text { Composite } \\
\text { Score }\end{array}$ \\
\hline (Ops-16) & (RI) & 10 & 3 & 4 & $\sqrt{ }$ & $\sqrt{ }$ & $\sqrt{ }$ & 4 & $\sqrt{ }$ & 25 \\
\hline (Ops-9) & (RI) & 8 & 5 & 4 & $\sqrt{ }$ & $\sqrt{ }$ & $\sqrt{ }$ & 4 & $\sqrt{ }$ & 25 \\
\hline (Ops-3) & (RI) & 8 & 5 & 4 & $\sqrt{ }$ & $\sqrt{ }$ & $\sqrt{ }$ & 3 & $\sqrt{ }$ & 24 \\
\hline (Ops-10) & (RI) & 8 & 5 & 4 & $\sqrt{ }$ & $\sqrt{ }$ & $\sqrt{ }$ & 4 & & 24 \\
\hline (Ops-9) & (OLE) & 7 & 5 & 4 & $\sqrt{ }$ & $\sqrt{ }$ & $\sqrt{ }$ & 4 & $\sqrt{ }$ & 24 \\
\hline (Ops-4) & (RI) & 7 & 4 & 4 & $\sqrt{ }$ & $\sqrt{ }$ & $\sqrt{ }$ & 4 & $\sqrt{ }$ & 23 \\
\hline (Ops-5) & (RI) & 7 & 4 & 4 & $\sqrt{ }$ & $\sqrt{ }$ & $\sqrt{ }$ & 3 & $\sqrt{ }$ & 22 \\
\hline (Ops-5) & (OLE) & 7 & 4 & 4 & $\sqrt{ }$ & $\sqrt{ }$ & $\sqrt{ }$ & 3 & $\sqrt{ }$ & 22 \\
\hline (Ops-6) & (RI) & 6 & 4 & 4 & $\sqrt{ }$ & $\sqrt{ }$ & $\sqrt{ }$ & 4 & $\sqrt{ }$ & 22 \\
\hline (Ops-13) & (RI) & 8 & 3 & 3 & $\sqrt{ }$ & $\sqrt{ }$ & $\sqrt{ }$ & 3 & $\sqrt{ }$ & 21 \\
\hline (Ops-1) & (OLE) & 6 & 5 & 4 & $\sqrt{ }$ & $\sqrt{ }$ & $\sqrt{ }$ & 3 & & 21 \\
\hline (Ops-6) & (OLE) & 6 & 5 & 3 & $\sqrt{ }$ & $\sqrt{ }$ & $\sqrt{ }$ & 3 & $\sqrt{ }$ & 21 \\
\hline \multicolumn{11}{|c|}{ Items in top 20 training priority ratings but not the highest composite scores } \\
\hline$(\mathrm{K}-2)$ & (OLE) & 8 & 6 & 5 & N/A & $\mathrm{N} / \mathrm{A}$ & N/A & N/A & $\mathrm{N} / \mathrm{A}$ & 19 \\
\hline$(\mathrm{K}-3)$ & (OLE) & 8 & 6 & 5 & N/A & N/A & $\mathrm{N} / \mathrm{A}$ & N/A & N/A & 19 \\
\hline (Lic-2) & (RI) & 7 & 3 & 3 & $\sqrt{ }$ & $\sqrt{ }$ & $\sqrt{ }$ & 3 & & 19 \\
\hline$(\mathrm{K}-3)$ & (RI) & 8 & 6 & 4 & N/A & N/A & N/A & N/A & N/A & 18 \\
\hline$(\mathrm{K}-4)$ & (RI) & 7 & 3 & 4 & $\sqrt{ }$ & & & 3 & & 18 \\
\hline (K-4) & (OLE) & 7 & 3 & 4 & $\sqrt{ }$ & & & 3 & & 18 \\
\hline$(\mathrm{K}-7)$ & (OLE) & 8 & 4 & 5 & N/A & N/A & $\mathrm{N} / \mathrm{A}$ & N/A & N/A & 17 \\
\hline (K-1) & (OLE) & 7 & 5 & 5 & N/A & N/A & N/A & N/A & N/A & 17 \\
\hline$(\mathrm{K}-2)$ & (RI) & 7 & 6 & 4 & N/A & N/A & $\mathrm{N} / \mathrm{A}$ & N/A & N/A & 17 \\
\hline$(\mathrm{K}-6)$ & (OLE) & 7 & 4 & 5 & N/A & N/A & N/A & N/A & N/A & 16 \\
\hline (K-6) & (RI) & 7 & 4 & 4 & N/A & N/A & N/A & N/A & N/A & 15 \\
\hline$(\mathrm{K}-7)$ & (RI) & 7 & 4 & 4 & N/A & N/A & N/A & N/A & N/A & 15 \\
\hline
\end{tabular}

Collectively, this information provides a framework for identifying the most impactful HSI systems and examining online survey ratings of impacts from their differences in fidelity on job performance and learning (see Figures 7.4 and 7.5). This links the inventory items to fidelity needs, albeit in an indirect and somewhat non-specific way. At minimum, it provides a basis for identifying which of the HSI systems warrant priority attention in a more detailed fidelity assessment, and informs the design of that assessment (for example, by identifying the type and level of knowledge and skill required by the Task/KSA Inventory items for which the system is important). Examining these data, for example, one can reasonably conclude that fidelity of the procedure system is considerably more important than of the workplace design, since the procedure system is rated as important or somewhat important to all but one of the top-rated KSA items listed, while workplace design received much lower scores.

As an example of how this information might be used to assess the implications for fidelity, Ops-10, Ops-3, and Ops-9, which address emergencies and automatic actuations (e.g., reactor trip), all have a DIF score of 8 , and a simulator necessity score of 5, indicating both a high priority for training and a high priority to use a simulator for training. To actually train the RI's with respect to these KSAs, the use of a simulator is almost certainly necessary, but this simulator does not necessarily need to be an exact replica of the RI's assigned plant CR. For these tasks, the RI is primarily observing and critiquing the 
performance of the operators, and is not getting very much information from the simulator itself. The RI will certainly use the simulator to check some critical parameters to verify that all control rods indicate fully inserted after a SCRAM, but will hear most critical parameters being reported verbally by the RO to the SRO, and therefore does not need to read them directly from the panels.

Table 8.3. Text of Task/KSA Inventory Items Included in Table 8.2

\begin{tabular}{|c|c|c|}
\hline $\begin{array}{c}\text { Task/KSA } \\
\text { Number }\end{array}$ & Task/KSA Summary & $\begin{array}{l}\text { Composite } \\
\text { Score }\end{array}$ \\
\hline $\begin{array}{l}\text { Ops-16 } \\
\text { (RI) }\end{array}$ & Gather information and communicate with the NRC during declared emergencies. & 25 \\
\hline Ops-9 (RI) & $\begin{array}{l}\text { Inspect, monitor, assess, and report on operator performance in obtaining, interpreting, } \\
\text { and properly using emergency operating procedures. }\end{array}$ & 25 \\
\hline Ops-3 (RI) & $\begin{array}{l}\text { Inspect, monitor, assess, and report on operator performance in reporting and } \\
\text { responding to automatic plant actuations. }\end{array}$ & 24 \\
\hline $\begin{array}{l}\text { Ops-10 } \\
\text { (RI) }\end{array}$ & $\begin{array}{l}\text { Inspect, monitor, assess, and report on operator performance in obtaining, interpreting, } \\
\text { and properly implementing the emergency classification guidelines and Emergency } \\
\text { Plan. }\end{array}$ & 24 \\
\hline $\begin{array}{l}\text { Ops-9 } \\
\text { (OLE) }\end{array}$ & $\begin{array}{l}\text { Administer, document, and justify a licensing evaluation of operator performance in } \\
\text { obtaining, interpreting, and properly using emergency operating procedures. }\end{array}$ & 24 \\
\hline $\begin{array}{l}\text { Ops-4a } \\
\text { (RI) }\end{array}$ & $\begin{array}{l}\text { Determine/verify operability, functionality, and availability of plant systems and } \\
\text { equipment and report findings to NRC. }\end{array}$ & 23 \\
\hline Ops-5 (RI) & $\begin{array}{l}\text { Inspect, monitor, assess, and report on operator performance in monitoring and cross- } \\
\text { checking diverse indications to verify and/or correctly understand plant conditions and } \\
\text { to identify potential instrument or equipment malfunctions. }\end{array}$ & 22 \\
\hline $\begin{array}{l}\text { Ops-5 } \\
\text { (OLE) }\end{array}$ & $\begin{array}{l}\text { Administer, document, and justify a licensing evaluation of operator performance in } \\
\text { monitoring and cross-checking diverse indications to verify and/or correctly } \\
\text { understand plant conditions and to identify potential instrument or equipment } \\
\text { malfunctions. }\end{array}$ & 22 \\
\hline $\begin{array}{l}\text { Ops-5 } \\
\text { (OLE) }\end{array}$ & $\begin{array}{l}\text { Administer, document, and justify a licensing evaluation of operator performance in } \\
\text { monitoring and cross-checking diverse indications to verify and/or correctly } \\
\text { understand plant conditions and to identify potential instrument or equipment } \\
\text { malfunctions. }\end{array}$ & 22 \\
\hline Ops-6 (RI) & $\begin{array}{l}\text { Inspect, monitor, assess, and report on operator performance in the use of plant } \\
\text { procedures to respond to instrument or equipment malfunctions. }\end{array}$ & 22 \\
\hline $\begin{array}{l}\text { Ops-13 } \\
\text { (RI) }\end{array}$ & $\begin{array}{l}\text { Inspect, monitor, assess, and report on operator performance in the use of procedures } \\
\text { for monitoring and operating systems for plant radiation control and release. }\end{array}$ & 21 \\
\hline $\begin{array}{l}\text { Ops-1 } \\
\text { (OLE) }\end{array}$ & $\begin{array}{l}\text { Administer, document, and justify a licensing evaluation of operator performance on } \\
\text { the use of control room indications to monitor and evaluate plant status and to identify } \\
\text { trends. }\end{array}$ & 21 \\
\hline $\begin{array}{l}\text { Ops-6 } \\
\text { (OLE) }\end{array}$ & $\begin{array}{l}\text { Administer, document, and justify a licensing evaluation of operator performance in } \\
\text { the use of plant procedures to respond to instrument or equipment malfunctions. }\end{array}$ & 21 \\
\hline \multicolumn{3}{|c|}{ Other Task/KSA Inventory Items with Training Priority Scores of 7 or Higher } \\
\hline K-2 (OLE) & $\begin{array}{l}\text { Knowledge of the components in the system and the effects a malfunction of system } \\
\text { component(s) would have on system performance, operation of the system, and of the } \\
\text { consequences (risk) associated with the malfunction. (17) }\end{array}$ & 17 \\
\hline $\mathrm{K}-3$ (OLE) & $\begin{array}{l}\text { Knowledge of the physical connections and cause-effect relations between the system } \\
\text { and other systems, including knowledge of the effect that loss or malfunction of the } \\
\text { system would have on other systems. }\end{array}$ & 17 \\
\hline
\end{tabular}


Table 8.3. (contd)

\begin{tabular}{|l|l|c|}
\hline $\begin{array}{c}\text { Task/KSA } \\
\text { Number }\end{array}$ & \multicolumn{1}{|c|}{ Task/KSA Summary } & $\begin{array}{c}\text { Composite } \\
\text { Score }\end{array}$ \\
\hline Lic-2 (RI) & $\begin{array}{l}\text { Inspect, monitor, assess, and report on the Licensee's compliance with NRC } \\
\text { regulatory requirements for operator performance in determining and implementing } \\
\text { the desired operational path forward based on plant status information and } \\
\text { administrative procedures and directives }\end{array}$ & 19 \\
\hline K-3 (RI) & $\begin{array}{l}\text { Knowledge of the physical connections and cause-effect relations between the system } \\
\text { and other systems, including knowledge of the effect that loss or malfunction of the } \\
\text { system would have on other systems. }\end{array}$ & 13 \\
\hline K-4 (RI) & $\begin{array}{l}\text { Knowledge of plant procedures, including administrative, normal, abnormal, and } \\
\text { emergency procedures, and the site Emergency Plan. }\end{array}$ & 18 \\
\hline K-4 (OLE) & $\begin{array}{l}\text { Knowledge of plant procedures, including administrative, normal, abnormal, and } \\
\text { emergency procedures, and the site Emergency Plan. }\end{array}$ & 18 \\
\hline K-7 (OLE) & $\begin{array}{l}\text { Knowledge of reactivity effects associated with various plant evolutions and the } \\
\text { impact the effects have on plant operations }\end{array}$ & 17 \\
\hline K-1 (OLE) & Knowledge of system function, design, and operation & 15 \\
\hline K-2 (RI) & $\begin{array}{l}\text { Knowledge of the components in the system and the effects a malfunction of system } \\
\text { component(s) would have on system performance, operation of the system, and of the } \\
\text { consequences (risk) associated with the malfunction. }\end{array}$ & 17 \\
\hline K-6 (OLE) & $\begin{array}{l}\text { Knowledge of reactivity effects associated with various plant evolutions and the } \\
\text { impact the effects have on plant operations }\end{array}$ & $\begin{array}{l}\text { Knowledge of the physical, chemical, thermal, and electrical properties and processes } \\
\text { of the plant and their impact on plant behavior. }\end{array}$ \\
\hline K-6 (RI) & \begin{tabular}{l} 
Know \\
\hline
\end{tabular} & \\
\hline
\end{tabular}

It is important not to forget the importance of the training and educational considerations beyond the scope of this report that also must be included in considerations of simulator fidelity needs and training effectiveness:

- differences in individual learning styles

- differences in background, particularly the presence or absence of CR experience

- differences in "types" of intelligences (verbal, mathematical, mechanical aptitude, etc.).

Trainers also must consider the experience profile of TTC students and the duration of their training in evaluating the level of fidelity needed to provide them with effective training. The experience profile of the students will vary, but does not necessarily include previous CR work experience. The initial training at TTC includes only 2 weeks of simulator training. If the student has little or no previous CR experience, 2 weeks is not enough time to go into great detail on control board manipulation or to learn skills to the point of automaticity. The student will be learning only general concepts and overall layout of the CR, and does not need to learn proficient control manipulation to do an inspector or examiner's job in any case. 
Table 8.4. Importance of HSI Systems to Task/KSA Items from Table 8.2

\begin{tabular}{|c|c|c|c|c|c|c|c|c|}
\hline 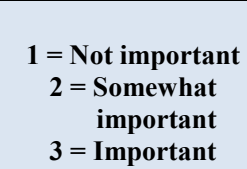 & $\begin{array}{l}\text { Procedure } \\
\text { System }\end{array}$ & $\begin{array}{c}\text { Instrumentation } \\
\text { System }\end{array}$ & $\begin{array}{l}\text { Control } \\
\text { Systems }\end{array}$ & $\begin{array}{c}\text { Dedicated } \\
\text { Safety- } \\
\text { Related } \\
\text { Controls and } \\
\text { Displays }\end{array}$ & $\begin{array}{l}\text { Displays of } \\
\text { Integrated } \\
\text { Information }\end{array}$ & $\begin{array}{l}\text { Alarms/ } \\
\text { Annunciato } \\
\text { r System }\end{array}$ & $\begin{array}{l}\text { Group- } \\
\text { view } \\
\text { Display } \\
\text { System }\end{array}$ & $\begin{array}{l}\text { Workplace } \\
\text { Design }\end{array}$ \\
\hline Ops-16 RI & 3 & 3 & 3 & 3 & 3 & 3 & 2 & 2 \\
\hline Ops-9 RI & 3 & 3 & 3 & 3 & 3 & 3 & 3 & 3 \\
\hline Ops-3 RI & 3 & 3 & 3 & 2 & 2 & 2 & 2 & 2 \\
\hline Ops-10 RI & 3 & 3 & 1 & 3 & 3 & 3 & 2 & 1 \\
\hline Ops-9 OLE & 3 & 3 & 3 & 3 & 3 & 3 & 3 & 3 \\
\hline Ops-4a RI & 2 & 3 & 2 & 3 & 2 & 2 & 1 & 1 \\
\hline Ops-5 RI & 2 & 3 & 2 & 3 & 3 & 2 & 2 & 1 \\
\hline Ops-5 OLE & 2 & 3 & 2 & 3 & 3 & 2 & 2 & 1 \\
\hline Ops-6 RI & 3 & 3 & 3 & 3 & 3 & 3 & 2 & 1 \\
\hline Ops-13 RI & 3 & 3 & 3 & 3 & 3 & 2 & 2 & 1 \\
\hline Ops-1 OLE & 1 & 3 & 1 & 3 & 3 & 2 & 3 & 2 \\
\hline Ops-6 OLE & 3 & 3 & 3 & 3 & 3 & 3 & 2 & 1 \\
\hline \multicolumn{9}{|c|}{ Items in top 20 training priority ratings but not the highest composite scores } \\
\hline K-2-OLE & \multicolumn{8}{|c|}{ Not rated because it is represents integrated knowledge } \\
\hline K-3-OLE & \multicolumn{8}{|c|}{ Not rated because it is represents integrated knowledge } \\
\hline Lic-2 RI & 3 & 3 & 3 & 3 & 3 & 3 & 3 & 1 \\
\hline K-3-RI & \multicolumn{8}{|c|}{ Not rated because it is represents integrated knowledge } \\
\hline K-4 RI & 3 & 1 & 1 & 1 & 1 & 1 & 1 & 2 \\
\hline K-4 OLE & 3 & 1 & 1 & 1 & 1 & 1 & 1 & 2 \\
\hline $\mathrm{K}-7-\mathrm{OLE}$ & \multicolumn{8}{|c|}{ Not rated because it is represents integrated knowledge } \\
\hline K-1-OLE & \multicolumn{8}{|c|}{ Not rated because it is represents integrated knowledge } \\
\hline K-2-RI & \multicolumn{8}{|c|}{ Not rated because it is represents integrated knowledge } \\
\hline K-6-OLE & \multicolumn{8}{|c|}{ Not rated because it is represents integrated knowledge } \\
\hline K-6-RI & \multicolumn{8}{|c|}{ Not rated because it is represents integrated knowledge } \\
\hline K-7-RI & \multicolumn{8}{|c|}{ Not rated because it is represents integrated knowledge } \\
\hline
\end{tabular}

\subsection{Study Conclusions}

The overall conclusion concerning physical fidelity needs for training NRC staff for advanced reactors is influenced by the evidence from the study that, although the current training program is brief and the TTC training simulators have only moderate physical fidelity with the CRs and simulators at which the trainees subsequently work, this training duration and moderate level of fidelity is sufficient, in the sense that the trainees go on to become successful OLE and RI personnel. The focus group respondents were generally satisfied with the current level of simulator fidelity, and (see Figures 7.6 and 7.7) at least $50 \%$ of respondents expressed the opinion that fidelity differences had no impact on difficulty of learning in their training (no more than $10 \%$ said it had a high impact) or their job performance.

The question of simulator fidelity requirements for initial training should be considered in the context of the experience level of the trainees, the number of days of training currently provided, and on-the-jobtraining that will be provided at their assigned facilities. While the experience level of initial trainees has historically varied, as noted in Section 6.3.1, a significant percentage of the NRC staff will become eligible to retire before 2011 (in conjunction with expected new reactor construction in the early 2010s), it is reasonable to expect that a significant percentage of the new OLE and RI hires will have little nuclear 
industry experience. This lack of experience will limit the amount of information they can absorb during a brief initial simulator training session. As discussed in Section 6.4, the study team identified no plans to fundamentally change the structure or duration of the TTC simulator training course. Given a mostly inexperienced trainee population receiving a 2-week course, as discussed in Chapter 2, it is reasonable to conclude that the finer points of a full-fidelity simulator would not provide great benefit to the trainees.

The nature of the on-the-job-training component of staff preparation also needs to be considered. Generally new OLE trainees give at least one exam under instruction with an experienced examiner, and must meet qualification requirements before working independently, and in any case will generally work as part of a team of two or three examiners, only becoming the senior examiner on a team after several years of experience. Likewise, new RI personnel receive OJT and are assigned to a plant with a senior colleague.

The nature of the jobs also limits the effect of simulator fidelity to any one particular plant. OLE personnel prepare an examination for one of several plants in their type (reactor vendor) over a period of several weeks, during which plant documentation is made available for preparation of the examination. The various BWR reactor plants, for example, vary from plant to plant at least as much as any one of them differs from the BWR simulator at the TTC. Similarly RIs typically move from one plant to another every few years and the plants at which they work are also likely to vary from each other as much or more than they vary from the TTC simulator used to train them. For both positions, therefore, the CRs and simulators of the plants at which the NRC personnel will work will vary over time, posing knowledge/ skill transfer challenges that will be somewhat offset by the opportunity to review documentation and work with more experienced colleagues.

The team understands that decisions concerning TTC simulator physical fidelity involve management as well as technical considerations. Cost-benefit tradeoffs, long-term maintenance and updating considerations, opportunistic availability of a simulator for sale, or the ability to order a simulator identical to one currently being built at a discount when new construction resumes, along with other factors, will need to be assessed during the decision-making process. The information provided in this report is intended to support inclusion of the technical aspects of these trade-offs against which these management decisions can be optimized.

\subsubsection{Summary of Findings}

\subsubsection{Training Needs}

The judgments of expert panels, the study team, and the NRC RIs and OLEs who responded to the on-line survey resulted in the following findings regarding training needs:

1. RIs and OLEs currently have a 2-week simulator training course once every 3 years for each reactor family. Simulator training courses are taken by RIs and OLEs only for reactor families associated with their current assignments.

2. It is expected that simulator training will be required for a potentially large number of new hires, many of whom may have no experience or familiarity with NPP CRs.

3. The high-level summary knowledge and task items that describe RI and OLE CR responsibilities developed by the study provides a basis for determining training needs and priorities. The Task/KSA 
Inventory items for RIs and OLEs include the vast majority of CR Task/KSA items required for all NRC inspection and oversight personnel. Each of the summary items can be placed in one of four categories: system knowledge, CR operations and indications tasks, operations management tasks, and plant license-related tasks. "System knowledge" items are the same for both RIs and OLEs. All other summary items are differentiated by position, with the preponderance of RI tasks focused on inspecting, monitoring, assessing, and reporting on operator performance and the preponderance of OLE tasks focused on administering, documenting, and justifying a licensing evaluation of operator performance on the same operator tasks. The Task/KSA Inventory items have been verified to be valid and comprehensive for both existing CRs and the CRs of new, evolutionary reactors.

4. While regulator tasks in the CRs of the new, evolutionary reactors are expected to remain the same as in current CRs, HSI technology in the new CRs is expected to be very different. The existing NRC staff who have received simulator training are generally unfamiliar with new CR technologies.

5. The Task/KSA Inventory items vary in their difficulty to perform, importance to safety, and frequency of occurrence, leading to a substantial variability in training priority (based on the DIF analysis) across the inventory items and the two positions analyzed. Of the high priority items, roughly half concerned system knowledge. These training priority ratings are independent of the training methodology that can be used to train these items, but can identify items for which effective training is particularly important.

6. Simulators were rated important for training RIs and OLEs for competent job performance for nearly half the Task/KSA Inventory items and not important for nearly one-third of the items. The importance of using a simulator varies little across the two positions. There is a moderate degree of correspondence between ratings of training priority and importance of simulators for training (e.g., simulators were rated of high importance for training on roughly half the items with a high training priority score).

7. Nearly all of the Task/KSA Inventory items require access to facts (i.e., basic, accepted information elements or details, terminology, definitions), both by memory and by external reference. Roughly one-third require that both the internal and external access to facts be quick.

8. Nearly all Task/KSA Inventory items require integration of a number of different types of conceptual knowledge about the system.

9. About one-fourth of the items require procedural knowledge (knowledge of how to do something) at the level of automaticity. Almost all of the remaining items require procedural knowledge to the level that the task can be executed, but not proficiently or efficiently.

10. None of the Task/KSA Inventory items require motor or tactile skills.

11. Most of the Task/KSA Inventory items require visual and auditory skills, but this is limited to a familiarity with how to execute the visual and auditory skills, rather than a requirement to reliably execute the skills.

12. At least one CR HSI system is important for performing every Task/KSA Inventory item. The importance of the eight different HSI systems varies across inventory items. The procedure system is important for $78 \%$ of the inventory items, while workplace design is important for $20 \%$ of the items. 
13. Information displays on visual display units (VDUs) will be the primary means through which operators interact with the plant in new CRs. CRs dependent on VDIs may present a large barrier to the evaluation of operator and crew performance because VDUs limit the ability of regulatory staff to observe operator behavior.

14. The information reflected above could be used in future decisions regarding the mix of simulator scope and fidelity that is appropriate for the training of various Tasks/KSAs. It could also be used to inform forthcoming analyses and decisions concerning simulator acquisition and training, addressing the scope, capabilities, and fidelity of the mix of simulators to be acquired for training regulatory oversight personnel for advanced reactors.

15. The information obtained from the online training and discussions with NRC staff indicate that the information contained in this study could be used to help trainees structure their on-the-job training experiences as well, particularly with regard to identifying and exploring fidelity differences between the training and reference simulators/CRs.

\subsubsection{Current Practice}

Discussions with NRC staff, analysis of available materials, and examination of results of the online survey of 74 current RIs and 23 current OLEs led to the following observations about current NRC simulator training practice.

1. Even though the physical fidelity of TTC simulators is fairly low with respect to the plants or sitespecific simulators to which they are assigned, these differences had little impact on the difficulty of learning during training or on job performance.

2. Physical fidelity differences are often addressed 'outside' the simulator by instructors, training materials, student discussions, or, as many current RIs and OLEs emphasized, by informal on-the-job training. The use of on-the-job training to supplement TTC simulator training was a common method discussed by many RIs and OLEs in focus groups and many RIs and OLEs view the training they receive from the NRC as a tool that prepares them to competently train themselves on-site at their assigned plants or site-specific simulators.

3. RIs and OLEs find existing TTC simulator training valuable for developing 1) knowledge of nuclear facility operations including system performance and interrelationships, 2) knowledge of operator roles and responsibilities in the CR during both normal operations and abnormal events, and 3) CR-related regulatory skills. These 3 items are inclusive of all 30 inventory items.

4. A prime benefit of TTC simulator training to RIs and OLEs is learning to integrate diverse knowledge that are required to understand the complex situations that may be encountered on their assignments.

5. A primary goal of simulator training is to teach general concepts that can be applied across reactors in the same family and to avoid teaching skills that are relevant only to specific CRs.

6. Interviews with FAA personnel responsible for simulator training of Aviation Safety Inspectors (ASIs) for the FAA's Flight Standards Service revealed that simulators with varying levels of fidelity are used for their training. High-fidelity simulators are used, but they may not match the particular airplanes an ASI has responsibility for inspecting. The requirement to use a simulator that matches a particular airplane is typically reserved for commercial airlines and depends on the complexity and distinctiveness of a particular cockpit that the ASI needs to be trained to inspect, the availability of 
time on high-fidelity simulators that match the cockpit, and the cost and time for training. All ASIs are trained using simulators for both traditional, analog HSIs in cockpits and for newer 'glass' cockpits.

7. A recent NRC study of computer-based HSI technologies relevant to new or modernized NPP CRs involving two foreign NPPs and three U.S. chemical manufacturing facilities (NRC 2002) revealed that interface management tasks, such as retrieving information, navigating displays, and manipulating windows, take a large amount of operator time. Though they are secondary to the primary tasks involved in process monitoring and control, interface management tasks are necessary to carry out the primary tasks. Interface management tasks for new CR displays were found to have potential negative effects on safety because the demands of interface management tasks may have negative effects on crew performance. Displays for new CRs can have hundreds or thousands of display pages; thus, retrieving information can be inherently problematic.

\subsection{Conclusions, Findings, and Recommendations}

The following list presents a summary of the conclusions, supportive findings, and team recommendations that resulted from this study.

1. Simulator training is imperative for nearly all tasks because:

- There is a need for integrated conceptual knowledge for nearly all tasks.

- Simulator training is effective for learning how to integrate conceptual knowledge.

- To the extent feasible, consider using simulators to train all tasks that require integrated conceptual knowledge, using the technical basis provided in this report as a guide.

2. There is no need for simulators with full physical fidelity because:

- Tasks cannot be trained to automaticity because of limitations in the amount of time available for simulator training.

- High-fidelity simulation has a high potential of interfering with learning and transfer since there is expected to be a large percentage of NPP CR novices and existing trainees who will have little familiarity with new CR technology.

- A lack of simulator fidelity in current TTC training does not appear to impact existing RI and OLE performance and can be addressed through means other than simulator training.

- RIs and OLEs are not responsible for operations and are not required to be trained to be able to operate a NPP CR. Though conventional wisdom in simulator training often points to the need for high levels of fidelity, high fidelity levels are only required when a high degree of fluent performance of procedural and psychomotor skills is necessary, as with NPP CR operators, who must be able to react correctly and quickly during abnormal or emergency events, and FAA ASI, who must be trained to flight standards on tasks that require a high degree of psychomotor control.

- Carefully assess whether any existing or future expectations for high levels of physical fidelity in training simulators are necessary. 
3. The full scope of CR HSI systems is required because:

- RIs and OLEs have tasks addressing emergency operating procedures.

- These tasks require the full scope of CR HSI systems for competent performance.

- These tasks are among the highest importance for training.

- These tasks benefit from simulator training since these tasks require integration of a large amount of conceptual knowledge of different types.

- Different reactor designs may require a different suite of HSI systems for their CRs. Consider making sure that their simulators include the full scope of HSI systems for all new CR designs that will be operating in the United States.

- The full scope of HSI systems for a single CR can conceivably be covered in multiple simulators including multiple 'full-scope' simulators and multiple 'part-task' simulators. If multiple simulators are used for training a task for a single CR design, assess the effects of using multiple simulators on the ability to train integrated conceptual knowledge. A potential option is to use part task simulators with high fidelity for some control HSI systems, where the technical basis supports this, combined with full-scope simulators that can use relatively low fidelity for HSI systems trained using higher fidelity simulators elsewhere.

- Functional HSI systems will likely have some level of integration between different HSI systems. The integration could range from having different HSI functions on the same display pages, to information flow between physically separate HSI systems, to different HSI systems being located within the same field of view. Any simulator solution considering omission of HSI integration should be evaluated for how necessary the omitted HSI integration would be for supporting training tasks effectively.

4. The potential for problems with negative transfer from simulator training exists because:

- Many staff needing simulator training will likely have no experience or familiarity with NPP CRs (F-6) or new CR HSI technology.

- Novice learners often learn to rely on perceptual cues inappropriately when there is a physical match between cues in the training context and the performance context.

- Indicate to trainees how the training simulator is different from the CR or site-specific simulators the trainee is assigned to, with emphasis on contexts in which there is a physical match between aspects of the HSIs but a functional difference.

- To 'un-train' improper fixedness on specific perceptual cues, consider training staff on the same tasks using multiple simulators with overlapping scope, but with differences in physical fidelity. The technical basis can be used to determine the scope of HSIs needed for training on particular tasks.

5. Part-task, high-fidelity simulators for training interface management tasks and for supplementing TTC simulator training is worth consideration:

- Display interface systems are likely to be standardized within a reactor design.

- Display interface systems are likely to be the primary method for accessing plant information, managing alarms and procedures, and implementing controls. These tasks are inherent in the vast 
majority of RI and OLE tasks. CR regulatory personnel will likely need to understand interface management tasks in order to evaluate operator behavior.

- Learning interface management tasks is difficult through observation alone.

- Interface management tasks are likely to be problematic for operator performance, and also may detract from NRC simulator training.

- Consider using part-task, high-fidelity simulators for training interface management tasks. Interface management tasks could conceivably be trained using simulators running on commonly available computers/operating systems and need not be hosted at a central training location.

6. Because the task and knowledge inventory for RIs and OLEs developed by this project is limited in detail and the analyses of physical fidelity needs and impacts are based on indirect measures (i.e., user and instructor reports), the study could not specifically determine the fidelity of the simulator HSI systems necessary for training each CR regulatory task for either current or advanced reactors.

However, the study team is confident that the information assembled by this study is valid and useful for informing decisions about the relative minimum physical fidelity of the HSI systems, as well as for the importance of each of the HSI system for teaching the tasks and the training priority of the tasks.

- The current expectation is that TTC simulator training provides training sufficient to prepare trainees to competently train themselves on-site and trainees can use means other than simulation training to adapt to site-specific differences. Because of ANSI/ANS-3.5-1998 requirements, RIs and OLEs will be observing operations in full-fidelity, full-scope CRs or CR simulators on-site.

- The current expectation for TTC simulator training is that it provides the means for trainees to learn to integrate knowledge learned elsewhere and that the trainees will learn general concepts that can be applied across reactors in the same family.

- Carry out a detailed analysis of each task to be trained using a simulator to precisely determine the physical fidelity needed for each HSI system.

- Precise physical fidelity is dependent on a complete design of CR HSIs, procedures, technical specifications, etc. To date, no new CR has a complete design. A detailed analysis of each CR design should be carried out to determine physical fidelity for each HSI system.

- The goals and expectations of current TTC simulator training have evolved based on a number of factors, some of those most likely being the variety of CR designs in operating reactors, even within the same reactor design, the availability and cost of NPP CR simulators, time constraints for simulator training, etc. Because there is expected to be much more CR standardization within a design and it may be more feasible to have simulators with higher levels of fidelity, consider if it is feasible to train the site-specific aspects of tasks, given available time and resource limitations and training priorities.

- Because precise fidelity requirements cannot currently be determined and CR HSI technology typically changes more frequently than other technology in a reactor, consider purchasing training simulator technology that can be easily adapted to approximate the precise fidelity requirements derived from specific CR designs. 


\subsubsection{Suggested Framework for Applying Study Findings to Training Simulator Physical Fidelity Decisions}

The information provided in this report provides a starting point for considerations of simulator training of RIs and OLEs for assignments to advanced reactors. Such considerations are recommended in the previous Section 8.2 on conclusions, findings and recommendations. In particular, the project team concludes that the Task/KSA Inventory; DIF analysis; and analysis of the importance of simulators to learning, difficulty of learning, and the type and level of knowledges and skills needed for each of the inventory items; supplemented with more specific information and management criteria; can assist in the analysis of simulator physical fidelity requirements. It could also be used to inform forthcoming analyses and decisions on simulator acquisition and training, in order to address the scope, capabilities and fidelity of the mix of simulators to be acquired for training regulatory oversight personnel for advanced reactors. The project team has assembled a schematic-level flow chart illustrating how this information could be incorporated into a decision-making process. This flow chart is shown in Figures 8.1 and 8.2.

Once tasks are selected for simulator training (Figure 8.1), each task should be studied to determine HSI systems important to its performance, the physical fidelity aspects of the systems, and the CR procedures that are important for the trainee to recognize during oversight. This information should then be used to guide determination of the scope and fidelity of simulation used for the training of the task (Figure 8.2). 


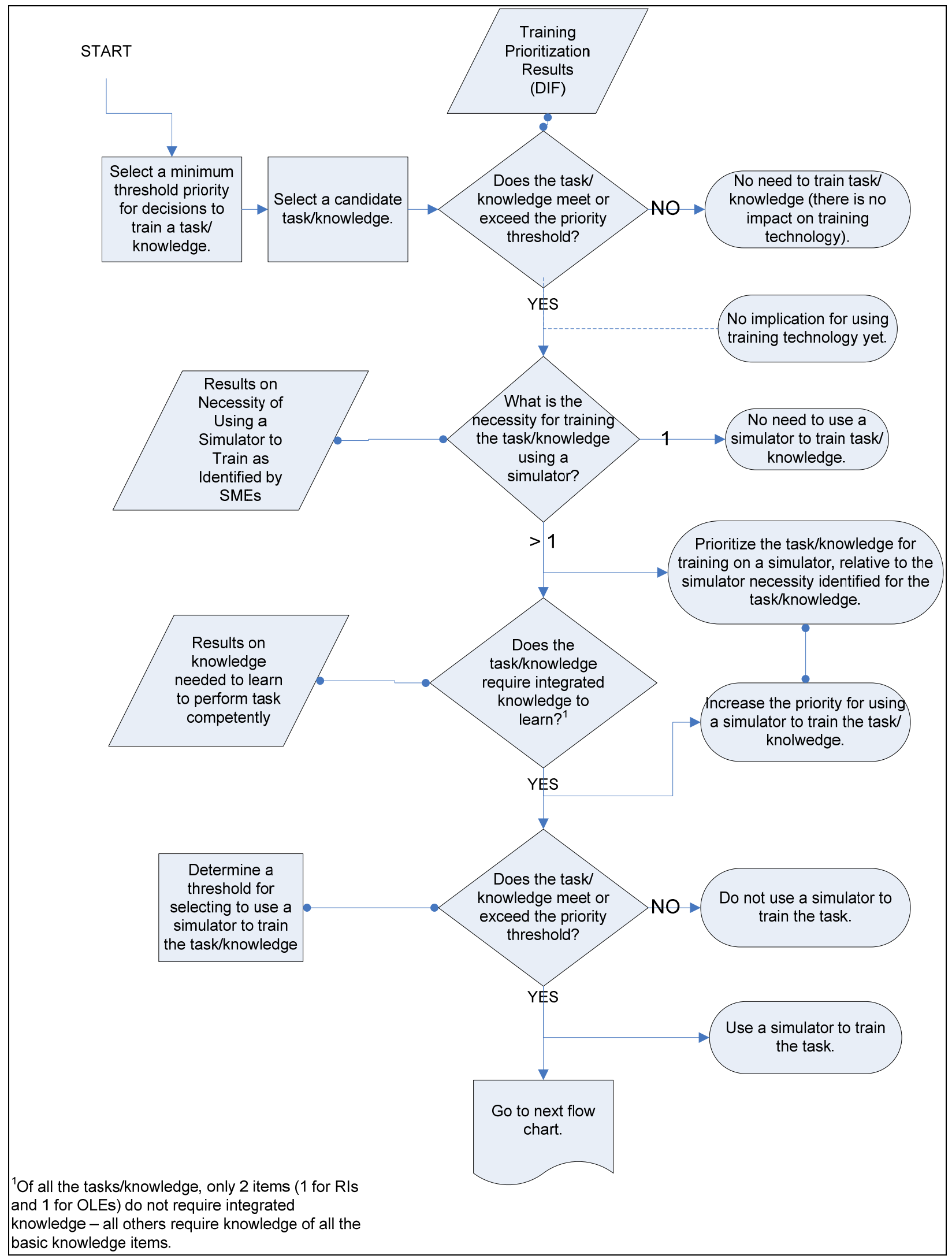

Figure 8.1. Flow Chart of Fidelity Needs Assessment (Part 1) 


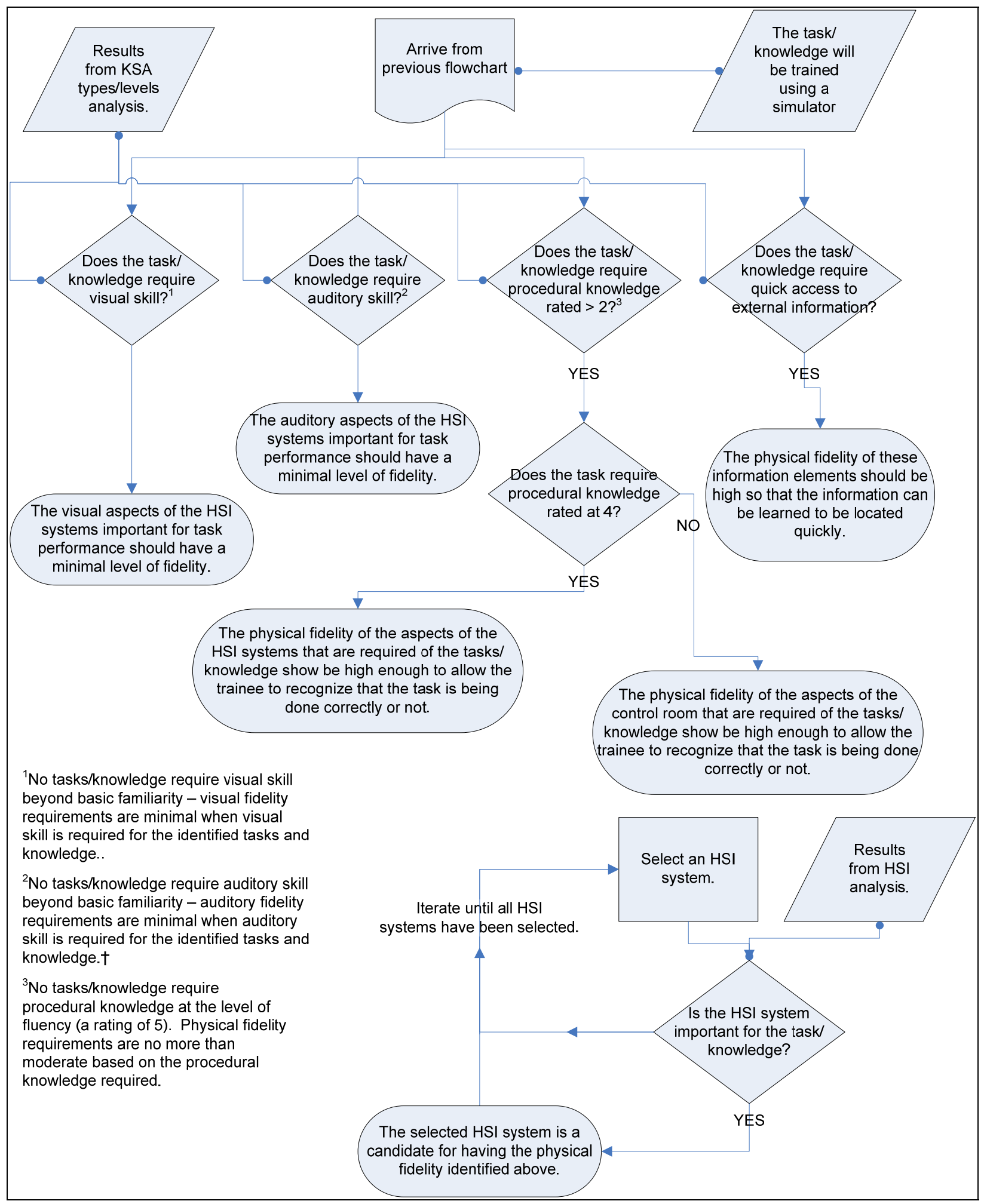

Figure 8.2. Flow Chart of Fidelity Needs Assessment (Part 2) 


\subsection{References}

10 CFR 55.45(a). 2009. “Operating Tests: Content.” Code of Federal Regulations. U.S. Nuclear Regulatory Commission.

10 CFR 55.46. 2009. "Simulation Facilities.” Code of Federal Regulations. U.S. Nuclear Regulatory Commission.

Adams J. 1987. "Historical Review and Appraisal of Research on the Learning, Retention, and Transfer of Human Motor Skills.” Psychological Bulletin 101:41-74.

Alessi SM. 1988. "Fidelity in the Design of Instructional Simulations." Journal of Computer Based Instruction 15:40-47.

Alexander AL, T Brunyé, J Sidman, and SA Weil. 2005. From Gaming to Training: A Review of Studies on Fidelity, Immersion, Presence, and Buy-in and Their Effects on Transfer in PC-Based Simulations and Games. DARWARS Training Impact Group. Accessed October 6, 2009, at http://www.darwars.bbn.com/downloads/DARWARS\%20Paper\%2012205.pdf.

American National Standard. 1998. Nuclear Power Plant Simulators for Use in Operator Training. ANSI/ANS-3.5-1998. Washington, D.C.

American National Standard. 1990. Nuclear Power Plant Simulators for Use in Operator Training. ANSI/ANS-3.5-1990. Washington, D.C.

Anderson LW and DR Krathwohl (eds.). 2001. "A Taxonomy for Learning, Teaching, and Assessing: A Revision of Bloom's Taxonomy of Educational Objectives.” Longman, New York, New York.

Anderson RJ. 1987. "The Reality Problem in Games and Simulations." In Simulation-Gaming in the Late 1980s: Proceedings of the International Simulation and Gaming Association's 17th International Conference, D Crookall et al. (eds.), pp. 43-50, Pergamon Press, Oxford.

Biggs J and K Collis. 1982. Evaluating the Quality of Learning: The SOLO Taxonomy (Structure of the Observed Learning Outcome). Academic Press, New York.

Buckley R and J Caple. 2007. The Theory and Practice of Training. 5th edition, Kogan Page, Philadelphia, Pennsylvania.

Carretta T and R Dunlap. 1998. Transfer of Training Effectiveness in Flight Simulation: 1986 to 1997. Air Force Research Laboratory, Mesa, Arizona.

Chi M, P Feltovich, and R Glaser. 1981. "Categorization and Representation of Physics Problems by Experts and Novices.” Cognitive Science: A Multidisciplinary Journal 5:121-152.

De Jong T and W Van Joolingen. 1998. "Scientific Discovery Learning with Computer Simulations of Conceptual Domains." Review of Educational Research 68(2):179. 
Feinstein A and H Cannon. 2001. "Fidelity, Verifiability, and Validity of Simulation: Constructs for Evaluation.” Developments in Business Simulation and Experiential Learning 28:57-67.

Fink CD and EL Shriver. 1978. "Simulators for Maintenance Training: Some Issues, Problems and Areas for Future Research.” Kinton, Inc., Alexandria, Virginia

Freda JS and H Ozkaptan. 1980. An Approach to Fidelity in Training Simulation. U.S. Army Research Institute for Behavioral and Social Sciences, Alexandria, Virginia.

Gerathewohl SJ. 1969. Fidelity of Simulation and Transfer of Training: A Review of the Problem. Clearinghouse for Federal Scientific and Technical Information, U.S. Office of Aviation Medicine, Springfield, Virginia, p. 18.

Gerjets PH and FW Hesse. 2004. "When Are Powerful Learning Environments Effective? The Role of Learner Activities and of Students' Conceptions of Educational Technology." International Journal of Educational Research 41(6):445-465.

Gick ML and KJ Holyoak. 1987. "The Cognitive Basis of Knowledge Transfer.” In Transfer of Learning: Contemporary Research and Applications, SM Cormier and HD Gick M and K Holyoak. 1983. "Schema Induction and Analogical Transfer." Cognitive Psychology 15:1-38.

Goldstone R and Y Sakamoto. 2002. "The Transfer of Abstract Principles Governing Complex Adaptive Systems." Cognitive Psychology 46:414-466.

Goldstone R and J Son. 2005. "The Transfer of Scientific Principles Using Concrete and Idealized Simulations." Journal of the Learning Sciences 14:69-110.

Gonzalez C and J Quesada. 2003. "Learning in Dynamic Decision Making: The Recognition Process.” Computational \& Mathematical Organization Theory 9:287-304.

Gore BF et al. 1984a. Knowledges and Abilities Required of Nuclear Power Plant Operators. Pressurized Water Reactors. PNL-5291, Pacific Northwest Laboratory, Richland, Washington. Subsequently published as NUREG-1122 by the U.S. Nuclear Regulatory Commission.

Gore BF et al. 1984b. Knowledges and Skills Required of Nuclear Power Plant Operators; A Synthesis from the INPO Jobs-Task Analysis. Vol. 1 and 2, PNL-5292, Pacific Northwest Laboratory, Richland, Washington.

Gredler M. 2004. "Games and Simulations and their Relationships to Learning." Handbook of Research on Educational Communications and Technology 571-581.

Gross DC. 1999. "Report from the Fidelity Implementation Study Group." Paper \# 99S-SIW-167, Proceedings of the Spring 1999 Simulation Interoperability Workshop, Simulation Interoperability and Standards Organization, Orlando, Florida.

Hamlin K. 2009. "Nuclear Education: The U.S. Experience." Presentation to the 1st National Meeting on Improving Education and Training for Chinese Nuclear Power Industry Personnel, May 25-29, 2009, 
Harbin, China. Accessed October 6, 2009, at

http://img.univs.cn/20090609/1/20090609115043722372499.pdf.

Harrow A. 1972. A Taxonomy of the Psychomotor Domain: A Guide for Developing Behavioral Objectives. McKay, New York, New York.

Hays RT. 1980. Simulator Fidelity: A Concept Paper. . U.S. Army Research Institute for Behavioral and Social Sciences, Alexandria, Virginia.

Hays RT and MJ Singer. 1989. Simulation Fidelity in Training System Design: Bridging the Gap between Reality and Training. Springer-Verlag, New York, New York.

Hays RT, JW Jacobs, P Carolyn, and E Salas. 1992a. "Flight Simulator Training Effectiveness: A MetaAnalysis." Military Psychology 4:63-72.

Hays RT, JW Jacobs, C Prince, and E Salas. 1992b. "Requirements for Future Research in Flight Simulation Training: Guidance Based on a Meta-Analytic Review.” The International Journal of Aviation Psychology 2:143-158.

Hmelo-Silver C and M Pfeffer. 2004. "Comparing Expert and Novice Understanding of a Complex System from the Perspective of Structures, Behaviors, and Functions.” Cognitive Science: A Multidisciplinary Journal 28:127-138.

International Atomic Energy Association (IAEA). 2007. Report of the Technical Meeting on Training for Regulatory Bodies in Member States with Nuclear Power Plants. December 17-19, 2007, Vienna, Austria. Accessed October 6, 2009, at http://www-ns.iaea.org/downloads/ni/training/report technicalmeeting_2007.pdf.

International Atomic Energy Agency (IAEA). 2004. Use of Control Room Simulators for Training of Nuclear Power Plant Personnel. IAEA-TECDOC-1411, September, Vienna, Austria.

International Atomic Energy Agency (IAEA). 2001. Training the Staff of the Regulatory Body for Nuclear Facilities: A Competency Framework. Vienna, Austria.

International Atomic Energy Agency (IAEA). 2000. Analysis Phase of Systematic Approach to Training (SAT) for Nuclear Plant Personnel. IAEA-TECDOC-1170, Nuclear Power Engineering Section, Vienna, Austria. Accessed October 6, 2009, at http://www-

pub.iaea.org/MTCD/publications/PDF/te_1170_prn.pdf.

International Atomic Energy Agency (IAEA). 1998. Selection, Specification, Design and Use of Various Nuclear Power Plant Training Simulators. Vienna, Austria.

Jones ER, RT Hennessy, and S Deutsch (eds.). 1985. "National Research Council Working Group on Simulation, Committee on Human Factors." Human Factors Aspects of Simulation. National Academy Press, Washington, D.C. 
Kaminiski J, V Sloutsky, and A Heckler. 2006. "Effects of Concreteness on Representation: An Explanation for Differential Transfer." In XXVIII Annual Conference of the Cognitive Science Society, R Sun and N Miyake (eds.), pp. 1581-1586.

Kinkade R and G Wheaton. 1972. "Training Device Design.” In Human Engineering Guide to Equipment Design, HP VanCott and RG Kinkade (eds.), pp. 667-699, American Institutes for Research, Washington, D. C.

Kyllonen P and E Alluisi. 1987. "Learning and Forgetting Facts and Skills." Handbook of Human Factors and Ergonomics, G Salvendy (ed.), John Wiley and Sons, New York, pp. 124-153.

Lever A. 2004. "Fidelity and Negative Training in System Simulation." In Simulation of On-board Systems. London: Royal Aeronautical Society.

Nuclear Energy Agency (NEA). 1998. Principal Working Group No. 1 - Extended Task Force on Human Factors, Task 5: Role of Simulators in Operator Training.

Nuclear Energy Agency (NEA)/CNRA/R. 1998. Working Group on Inspection Practices (WGIP). Comparison of the Inspection Practices in Relation to the Control Room Operator and Shift Supervisor Licenses. Paris.

NWI, Inc. 2008. "Nuclear Resurgence: Training and Infrastructure to Accomplish Reactor Inspections and Operator Licensing." NWI Newsletter 4(4). Accessed October 6, 2009, at nwiservices.us/Fall\%202008\%20NWI\%20Newsletter.pdf.

Patrick J. 2003. "Training." Chapter 11 in Principles and Practice of Aviation Psychology. PS Tsang and MA Fidulich (eds.), Human Factors in Transportation Series, pp. 397-434, Lawrence Erlbaum, Mahwah, New Jersey.

Piskurich GM, P Beckschi, and B Hall (eds.). 2000. The ASTD Handbook of Training Design and Delivery. Adobe eBook.

Porter A. 2002. "Measuring the Content of Instruction: Uses in Research and Practice." Educational Researcher 31(7):3.

Quesada J, W Kintsch, and E Gomez. 2003. "Latent Problem Solving Analysis as an Explanation of Expertise Effects in a Complex, Dynamic Task." In Twenty-Fifth Annual Conference of the Cognitive Science Society. R Alterman and D Kirsh (eds.), Lawrence Erlbaum Associates, July 31-August 2, 2003, Boston, Massachusetts.

Rankin W, P Bolton, R Shikiar, and L Saari. 1984a. Nuclear Power Plant Simulators for Operator Licensing and Training. Part I. The Need for Plant-Reference Simulators. Part II. The Use of PlantReference Simulators. Battelle Human Affairs Research Centers, Seattle, Washington.

Rankin W, P Bolton, R Shikiar, and L Saari. 1984b. Simulator Fidelity and Training Effectiveness: A Comprehensive Bibliography with Selected Annotations. Battelle Human Affairs Research Centers, Seattle, Washington. 
Reder LM and RL Klatzky. 1994. “The Effect of Context on Training: Is Learning Situated?” In Learning, Remembering, Believing: Enhancing Human Performance. D Druckman and RA Bjork (eds.), pp. 25-56, National Academy Press, Washington, D.C.

Roscoe SN. 1991. "Simulator Qualification: Just as Phony as It Can Be." The International Journal of Aviation Psychology 1:335-339.

Scerbo M and S Dawson. 2007. "High Fidelity, High Performance? Simulation in Healthcare." The Journal of the Society for Simulation in Healthcare 2:224.

Scheiter K, P Gerjets, T Huk, B Imhof, and Y Kammerer. 2009. "The Effects of Realism in Learning with Dynamic Visualizations." Learning and Instruction 19:481-494.

Schneider W. 1985. “Training High-Performance Skills: Fallacies and Guidelines.” Human Factors 27:285-300.

Schwartz DL and NI Nasir. 2008. Learning: Transfer of Learning. Answers.com.

Shriffrin RM and W Schneider. 1977. "Controlled and Automatic Human Information Processing. II. Perceptual Learning, Automatic Attending and a General Theory." Psychological Review 84(2):127-190.

Slenker K and BW Cream. 1977. Part-Task Trainer for the F-106A MA-1 Radar/Infrared Fire Control System: Design, Specification, and Operation. Air Force Human Resources Laboratory, Advanced Systems Division, Wright-Patterson Air Force Base, Ohio.

Stewart J, D Johnson, and W Howse. 2008. Fidelity Requirements for Army Aviation Training Devices: Issues and Answers. Fort Rucker, Alabama.

Su YLD. 1984. "A Review of the Literature on Training Simulators: Transfer of Training and Simulator Fidelity.” Technical Report No. 84-1. Arlington, Virginia.

U.S. Department of Energy. 1995. Alternative Systematic Approaches to Training. DOE Handbook, Washington, D.C.

U.S. Department of Energy. 1994. Training Program Handbook: A Systematic Approach to Training. DOE Handbook-1078-94, Washington, D.C.

U.S. Government Accountability Office (GAO). 2007. Human Capital: Retirements and Anticipated New Reactor Applications Will Challenge NRC's Workforce. Report to the Subcommittee on Oversight of Government Management, the Federal Workforce, and the District of Columbia, Committee on Homeland Security and Governmental Affairs, U.S. Senate from http://www.gao.gov/products/GAO-07105.

U.S. Nuclear Regulatory Commission (NRC). 2009. ADM 109: Training, Development, and Qualification Programs. Nuclear Security and Incident Response, Washington, D.C. 
U.S. Nuclear Regulatory Commission (NRC). 2008 (July 3). Training and Infrastructure Needs to Accomplish New Reactor Inspection Licensing. SECY-08-0096. Accessed October 6, 2009, at http://www.nrc.gov/reading-rm/doc-collections/commission/secys/2008/secy2008-0096/20080096scy.html.

U.S. Nuclear Regulatory Commission (NRC). 2007. Knowledge and Abilities Catalog for Nuclear Power Plant Operators: Boiling Water Reactors. NUREG-1123, Rev. 2, Supplement 1, Washington, D.C.

U.S. Nuclear Regulatory Commission (NRC). 2007. Knowledge and Abilities Catalog for Nuclear Power Plant Operators: Pressurized Water Reactors. NUREG-1122, Rev. 2, Supplement 1, Washington, D.C.

U.S. Nuclear Regulatory Commission (NRC). 2004. Human Factors Engineering (HFE) Program Review Model. NUREG-0711, Rev. 2. Prepared by JM O'Hara, JC Higgins, JJ Persensky, PM Lewis, and JP Bongarra, Brookhaven National Laboratory. Upton, New York.

U.S. Nuclear Regulatory Commission (NRC). 2004. Operator Licensing Examination Standards for Power Reactors. NUREG-1021, Rev. 9. Prepared by S Guenther, Washington, D.C.

U.S. Nuclear Regulatory Commission (NRC). 2002. Human-System Interface Design Review Guidelines. NUREG-0700, Rev. 2. Prepared by JM O’Hara, WS Brown, PM Lewis, and JJ Persensky, Brookhaven National Laboratory, Upton, New York.

U.S. Nuclear Regulatory Commission (NRC). 2001. Regulatory Guide 1.149 rev 3 - Nuclear Power Plant Simulation Facilities for Use in Operator Training and License Examinations. Washington, D.C.

U.S. Nuclear Regulatory Commission (NRC). 1983. Regulatory Guide 1.97 rev 3. Instrumentation for Light-Water-Cooled Nuclear Power Plants to Assess Plant and Environs Conditions During and Following an Accident. December 1975, (Rev. 1) August 1977 [8001240572], (Rev. 2) December 1980 [7912310387], (Rev. 3) May 1983 [8502060303]. Washington, D.C.

Wheaton G, A Rose, P Fingerman, A Korotkin, D Holding, and A Mirabella. 1976. Evaluation of the Effectiveness of Training Devices: Literature Review and Preliminary Model. Ari Research Memorandum 76-6, Army Research Institute for the Behavioral and Social Services, Alexandria, Virginia.

Wilson LO. 2006. Beyond Bloom - A New Version of the Cognitive Taxonomy. Accessed October 6, 2009, at http://www.uwsp.edu/education/lwilson/curric/newtaxonomy.htm

Wogman NA, LJ Bond, AE Waltar, and RE Leber. 2005. "The Nuclear Education and Staffing Challenge: Rebuilding Critical Skills in Nuclear Science and Technology." Journal of Radioanalytical and Nuclear Chemistry 263(1):138.

World Nuclear Association. September 2009. Advanced Nuclear Power Reactors. Accessed October 6, 2009, at http://world-nuclear.org/info/inf08.html. 


\subsection{Bibliography}

Adamy DL. 2003. Introduction to Electronic Warfare Modeling and Simulation. Artech House, Boston, Massachusetts.

Adams J. 1979. “On the Evaluation of Training Devices.” Human Factors 21:711-720.

Albro E, D Uttal, J DeLoache, JA Kaminski, VM Sloutsky, AF Heckler, B Rittle-Johnson, J Star, R Goldstone, and J Son. 2007. "Fostering Transfer of Knowledge in Education Settings." In 29th Annual Conference of the Cognitive Science Society, Nashville, Tennessee.

Alessi SM. 2000. "Simulation Design for Training and Assessment." In Aircrew Training and Assessment, DH Andrews and HF O’Neil Jr. (eds.), Lawrence Erlbaum Associates, Mahwah, New Jersey.

Allen J, L Buffardi, and R Hays. 1998. The Relationship of Simulator Fidelity to Task and Performance Variables. Prepared by George Mason University Department of Psychology for the U.S. Department of the Army, Defense Technical Information Center. Accessed October 6, 2009, at http://handle.dtic.mil/100.2/ADA238941.

Allen JA and RT Hays. 1984. "Maintenance Simulator Fidelity in Electromechanical Troubleshooting Training." Presentation to the $92^{\text {nd }}$ Annual Convention of the American Psychological Association, August 24-28, 1984, Toronto, Ontario, Canada.

Allen JA, RT Hays, and LC Buffardi. 1986. "Maintenance Training Simulator Fidelity and Individual Differences in Transfer of Training." Human Factors 28:497-509.

Ames Research Center. 1985. "Training of Nuclear Facility Personnel: Proceedings of the Sixth Symposium.” In NASA Technical Memorandum 104016. National Technical Information Service, Moffett Field, California.

Andrews DH. 1988. "Relationships among Simulators, Training Devices, and Learning: A Behavioral View.” Educational Technology 28:48-54.

Andrews DH, LA Carroll, and HH Bell. 1995. "The Future of Selective Fidelity in Training Devices." Educational Technology 35:32-36.

Atencio A, Ames Research Center, and U.S. Army Aviation and Troop Command. 1993. Fidelity Assessment of a Uh-60a Simulation on the NASA Ames Vertical Motion Simulator.

Badariah S and K Mania. 2005. "The Effect of Visual Fidelity on Transfer of Training and Awareness States." In Proceedings of the 2nd Symposium on Applied Perception in Graphics and Visualization, pp. 296-310, ACM Siggraph Press, Spain.

Baudhuin E. 1987. "The Design of Industrial and Flight Simulators." In Transfer of Learning, SM Cormier and JD Hagman (eds.), pp. 217-237. Academic Press, New York. 
Baum DR. 1982. Specification of Training Simulator Fidelity: A Research Plan. Technical Report 558, Army Research Institute for Behavioral and Social Sciences, Alexandria, Virginia.

Baumont G and BG Montes. 1997. "Current Practices in Operator Training with Simulators in OECD Countries." In Human Factors and Power Plants, 1997. "Global Perspectives of Human Factors in Power Generation.” Proceedings of the 1997 IEEE Sixth Conference on Human Factors and Power Plants, pp. 17/11-17/16.

Beaubien JM and DP Baker. 2004. "The Use of Simulation for Training Teamwork Skills in Health Care: How Low Can You Go?” Quality \& Safety in Health Care 13(Suppl 1):i51-i56.

Bell HH and WL Waag. 1998. "Evaluating the Effectiveness of Flight Simulators for Training Combat Skills: A Review." International Journal of Aviation Psychology 8:223.

Berry D. 1991. "The Role of Action in Implicit Learning." The Quarterly Journal of Experimental Psychology Section A 43:881-906.

Billett S. 1994. "Situated Learning: A Workplace Experience." Australian Journal of Adult and Community Education 34:112-130.

Blaiwes AS and RT Hays. 1998. A Simulation and Training Technology Survey. Naval Air Warfare Center Training Systems Division, Orlando, Florida.

Blaiwes AS and JJ Regan. 1986. "Training Devices: Concepts and Progress." In Military Contributions to Instructional Technology, JA Ellis (ed.). Praeger Publishers, New York, New York.

Bradshaw MJ, B Fuszard, and AJ Lowenstein. 2001. Fuszard's Innovative Teaching Strategies in Nursing. Aspen Publishers, Gaithersburg, Maryland.

Bradshaw MJ and AJ Lowenstein. 2007. Innovative Teaching Strategies in Nursing and Related Health Professions. Jones and Bartlett Publishers, Sudbury, Massachusetts.

Brown J, A Collins, and P Duguid. 1989. "Situated Cognition and the Culture of Learning." Educational Researcher 18:32.

Bruno RJ. 1985. "Integrating a Non-Plant Referenced Simulator into the Training Process." In Proceedings of the Sixth Symposium on Training of Nuclear Facility Personnel, Nashville, Tennessee.

Bunker WM. 1978. “Training Effectiveness Versus Simulation Realism.” In Eleventh NTEX/Industry Conference. Naval Training Equipment Center, Orlando, Florida.

Chamberlain HD. 2007. "FAA TAA Inspector Training.” FAA Aviation News.

Chidester T, C Hackworth, and W Knecht. 2007. Participant Assessments of Aviation Safety Inspector Training for Technically Advanced Aircraft. Oklahoma City, Oklahoma. 
Cox JA, R Wood, L Boren, and HW Thorne. 1965. Functional and Appearance Fidelity of Training Devices for Fixed Procedures Tasks. Prepared by George Washington University Human Resources Research Office for the Defense Technical Information Center.

Crawford M. 1966. "Dimensions of Simulation.” American Psychologist 21:788-796.

Cross DC. 1999. "Report from the Fidelity Implementation Study Group." In Spring Simulation Interoperability Workshop. Accessed October 6, 2009, at http://vva.msco.mil/Ref_Docs/Ref_Docs/99ssiw-167.pdf.

Day DL and DK Kovacs. 1996. Computers, Communication and Mental Models. Taylor \& Francis, London; Bristol, Pennsylvania.

Dennis KA and D Harris. 1998. "Computer-Based Simulation as an Adjunct to Ab Initio Flight Training.” International Journal of Aviation Psychology 8:261-276.

Dieckmann P, T Manser, and T Wehner. 2003. "Presence and High Fidelity Patient Simulators in Anaesthesiology: Influences Derived from Interviews and Questionnaires." In 6th International Workshop on Presence, October 6-8, 2003, Aalborg University, Denmark.

Donchin E, M Fabiani, and A Sanders. 1989. "The Learning Strategies Program: An Examination of the Strategies in Skill Acquisition.” Acta Psychologica 71:17-22.

Dunn WF. 2004. Simulators in Critical Care Education and Beyond. Society of Critical Care Medicine, Des Plaines, Illinois.

Dwyer FM. 1974. Strategies for Improving Visual Learning. Learning Services, State College, Pennsylvania.

Ellis D, J Matter, and R Duggan. 2008. "Training Programmes for the Systems Approach to Nuclear Security.” International Journal of Nuclear Knowledge Management 3(1):6-16.

Estock J, D Alexander, K Gildea, C Nash, and B Blueggel. 2006. "A Model-Based Approach to Simulator Fidelity and Training Effectiveness." In The Interservice/Industry Training, Simulation, and Education Conference. Vol. 2006. National Training Systems Association (NTSA).

Feinstein AH and HM Cannon. 2002. "Constructs of Simulation Evaluation.” Simulation Gaming $33: 425-440$.

Fisk AD and WA Rogers. 1988. "The Role of Situational Context in the Development of HighPerformance Skills.” Human Factors 30:703-712.

Foshay WR. 2006. "Building the Wrong Simulation: Matching Instructional Intent in Teaching Problem Solving to Simulation Architecture.” Technology, Instruction, Cognition and Learning 3:63-72.

Fowlkes J, DJ Dwyer, RL Oser, and E Salas. 1998. "Event-Based Approach to Training (EBAT)." International Journal of Aviation Psychology 8:209-221. 
Frederiksen TR and BY White. 1989. "An Approach to Training Based Upon Principled Task Decomposition.” Acta Psychologica 71:89-146.

Gagne RM. 1954. "Training Devices and Simulators: Some Research Issues.” The American Psychologist 9:95-107.

Gallagher J. 1994. “Teaching and Learning: New Models.” Annual Review of Psychology 45:171-195.

Glaser R. 1989. "Expertise and Learning: How Do We Think About Instructional Processes Now That We Have Discovered Knowledge Structures." In Complex Information Processing: The Impact of Herbert A. Simon, D Klahr and K Kotovsky (eds.), pp. 269-282, Erlbaum \& Associates, Hillsdale, New Jersey.

Goodrich KH. 1993. "A High-Fidelity, Six-Degree-of-Freedom Batch Simulation Environment for Tactical Guidance Research and Evaluation." In NASA Technical Memorandum 4440, U.S. National Aeronautics and Space Administration Scientific and Technical Information Program, Springfield, Virginia.

Goodstein L. 1986. Training Diagnostic Skills for Nuclear Power Plants: Final Report of the NKA Project Lit-4. Copenhagen.

Gopher D, M Weil, and T Bareket. 1994. "Transfer of Skill from a Computer Game Trainer to Flight." Human Factors 36:387-406.

Griffiths JR, RJ Hartley, and JP Willson. 2002. "An Improved Method of Studying User-System Interaction by Combining Transaction Log Analysis and Protocol Analysis." Information Research 7(4). Accessed October 6, 2009, at http://informationr.net/ir/7-4/paper139.html.

Hale AF and MC Hugh. 2001. "Fidelity, Verifiability, and Validity of Simulation: Constructs for Evaluation.” Developments in Business Simulation and Experiential Learning 28:57-67.

Hammell TJ, KE Williams, JA Grasso, W Evans. 1980. Simulators for Mariner Training and Licensing: Phase 1: Role of Simulators in the Mariner Training and Licensing Process. National Technical Information Service, Kings Point, New York, Springfield, Virginia.

Hays RT. 1992. "Systems Concepts for Training Systems Development." IEEE Transactions on Systems, Man, and Cybernetics 22(2):258-266.

Hays RT. 1981. Training Simulator Fidelity Guidance: The Iterative Data Base Approach. U.S. Army Research Institute for Behavioral and Social Sciences, Alexandria, Virginia.

Hesketh B. 1997. "Dilemmas in Training for Transfer and Retention.” Applied Psychology: An International Review 46(4):317-386.

Issenberg SB, WC McGaghie, ER Petrusa, DL Gordon, and RJ Scalese. 2005. "Features and Uses of High-Fidelity Medical Simulations That Lead to Effective Learning: A BEME Systematic Review." Medical Teacher 27(1):10-28. 
Jentsch F and CA Bowers. 1998. "Evidence for the Validity of PC-Based Simulations in Studying Aircrew Coordination.” The International Journal of Aviation Psychology 8(3):243-260.

Kaiser MK and JA Schroeder. 2003. "Flights of Fancy: The Art and Science of Flight Simulation." Chapter 12 in Principles and Practice of Aviation Psychology, PS Tsang and MA Fidulich (eds.), Human Factors in Transportation Series, Lawrence Erlbaum Mahwah, New Jersey, pp. 435-472.

Lammers RL. 2007. "Simulation: The New Teaching Tool." Annals of Emergency Medicine 49:505-507.

Lane NE and EA Alluisi. 1992. Fidelity and Validity in Distributed Interactive Simulation: Questions and Answers. Institute for Defense Analysis, Alexandria, Virginia.

Lintern G, D Sheppard, D Parker, K Yates, and M Nolan. 1989. "Simulator Design and Instructional Features for Air-to-Ground Attack: A Transfer Study.” Human Factors 31:87-99.

Longridge T, J Burki-Cohen, T Go, and A Kendra. 2001. "Simulator Fidelity: Considerations for Training and Evaluation of Today's Airline Pilots." In The 11th International Symposium on Aviation Psychology. Columbus, Ohio.

Lowenstein AJ, MJ Bradshaw, and B Fuszard. 2001. Fuszard's Innovative Teaching Strategies in Nursing. Aspen Publishers, Gaithersburg, Maryland.

Mania K, T Troscianko, R Hawkes, and A Chalmers. 2003. "Fidelity Metrics for Virtual Environment Simulations Based on Spatial Memory Awareness States.” Presence: Teleoperators \& Virtual Environments 12:296-310.

Manning CA. 2000. Measuring Air Traffic Controller Performance in a High-Fidelity Simulation. Federal Aviation Administration Civil Aeromedical Institute, Oklahoma City, Oklahoma.

Mansur MH. 1992. Modeling Methods for High-Fidelity Rotorcraft Flight Mechanics Simulation Microform. United States Army Aviation Research and Technology Activity, Ames Research Center, Moffett Field, California.

McCauley ME. 2006. "Do Army Helicopter Training Simulators Need Motion Bases?” Technical Report 1176. U.S. Naval Postgraduate School Department of Operations Research and Administrative Sciences, U.S. Army Research Institute for the Behavioral and Social Sciences Rotary-Wing Aviation Research Unit, Fort Rucker, Alabama.

McElroy AD and C-C Pan. 2009. "Effectiveness of Software Training Using Simulations: An Exploratory Study.” Performance Improvement Quarterly 21(4):35-53.

Miller GG. 1974. Some Considerations in the Design and Utilization of Simulators for Technical Training. Air Force Human Resources Laboratory, Brooks Air Force Base, Texas.

Miller RB. 1954. Psychological Considerations in the Design of Training Equipment. Report No. 54-563, Wright Air Development Center, Wright-Patterson Air Force Base, Ohio. 
Milrad M, J Spector, and P Davidsen. 2000. "Building and Using Simulation Based Environments for Learning About Complex Domains.” In M/SET 2000, AACE Press, pp. 304-308.

Mitsubishi Heavy Industries, Ltd. 2007. HSI System Description and HFE Process. MUAP-07007-NP, Rev. 1, US-APWR Topical Report, Tokyo, Japan.

Naylor JC and GE Briggs. 1963. "Effects of Task Complexity and Task Organization on the Relative Efficiency of Part and Whole Training Methods." Journal of Experimental Psychology 65:217-224.

Noble C. 2002. "The Relationship between Fidelity and Learning in Aviation Training and Assessment." Journal of Air Transportation World Wide 7:33-54.

Norman DA and DG Bobrow. 1975. "On Data-Limited and Resource-Limited Processes." Cognitive Psychology 7(1):44-64.

Orlansky J and J String. 1977. Cost Effectiveness of Computer-Based Instruction in Military Training (Ida Paper P-1375). Institute for Defense Analyses, Arlington, Virginia.

O’Sullivan, C, J Dingliana, T Giang, and MK Kaiser. 2003. "Evaluating the Visual Fidelity of Physically Based Animations.” ACM Transactions on Graphics 22(3):527-536.

Park B and H Qi. 2004. Development and Evaluation of a Calibration and Validation Procedure for Microscopic Simulation Models. Virginia Transportation Research Council, Charlottesville, Virginia.

Perkins DN. 2008. Making Learning Whole: How Seven Principles of Teaching Can Transform Education. Jossey-Bass, San Francisco, California.

Pfeiffer MG, JD Horey, and SK Butrimas. 1991. "Transfer of Simulated Instrument Training to Instrument and Contact Flight.” International Journal of Aviation Psychology I:219-229.

Proceedings of the Symposium on Training of Nuclear Facility Personnel. 1989. National Technical Information Service, April 14-27, 1989, Gatlinburg, Tennessee.

Prophet WW and HA Boyd. 1970. Device-Task Fidelity and Transfer of Training: Aircraft Cockpit Procedures Training. National Technical Information Service, Operations Division, Springfield, Virginia.

Rehmann AJ, R Mitman, and M Reynolds. 1995. A Handbook of Flight Simulation Fidelity Requirements for Human Factors Research. Technical Report No. DOT/FAA/CT-TN95/46, Crew Systems Ergonomics Information Analysis Center, Wright-Patterson Air Force Base, Ohio.

Reigeluth CM. 1999. Instructional-Design Theories and Models. Volume Ii, A New Paradigm of Instructional Theory. Lawrence Erlbaum Associates, Mahwah, New Jersey.

Roscoe SN. 1971. “Incremental Transfer Effectiveness.” Human Factors 13(6):561-567.

Rosenbaum DA, RA Carlson, and RO Gilmore. 2001. "Acquisition of Intellectual and Perceptual-Motor Skills." Annual Review of Psychology 52:453-470. 
Roza ZC, DC Gross, and SY Harmon. 2001. "Report Out of the Fidelity Experimentation Implementation Study Group.” In 2000 Spring Simulation Interoperability Workshop, Simulation Interoperability Standards Organization, Orlando, Florida.

Salas E and JA Cannon-Bowers. 2001. "The Science of Training: A Decade of Progress." Annual Review of Psychology 52:471-499.

Salas E, HA Priest, KA Wilson, and CS Burke. 2006. "Scenario-Based Training: Improving Military Mission Performance and Adaptability." In Military Life: The Psychology of Serving in Peace and Combat, Vol. 2: Operational Stress, edited by TW Britt, AB Adler, and CA Castro, Praeger Security International, Westport, Connecticut.

Salas E, CA Bowers, and C Prince. 1998. "On Simulation and Training in Aviation.” International Journal of Aviation Psychology 8(3):195-196.

Salas E, CA Bowers, and L Rhodenizer. 1998. "It Is Not How Much You Have but How You Use It: Toward a Rational Use of Simulation to Support Aviation Training." International Journal of Aviation Psychology 8(3):197-208.

Shebilske WL, JA Jordan, BP Goettl, and LE Paulus. 1998. “Observation Versus Hands-on Practice of Complex Skills in Dyadic, Triadic, and Tetradic Training-Teams." Human Factors 40(4):521-525.

Simons P. 1999. “Transfer of Learning: Paradoxes for Learners.” International Journal of Educational Research 31:577-589.

Son JY and RL Goldstone. 2004. "The Role of Perceptual Concreteness in Analogical Mapping and Transfer." In Twenty-Fifth Annual Conference of the Cognitive Science Society. R Alterman and D Kirsh (eds.), Lawrence Erlbaum Assoc Inc., July 31-August 2, 2003, Boston, Massachusetts.

Spears WD. 1983. Processes of Skill Performance: A Foundation for the Design and Use of Training Equipment. Prepared by Seville Training Systems Corporation for Defense Technical Information Center, Pensacola, Florida.

Stanton N. 1996. "Simulators: A Review of Research and Practice." In Human Factors in Nuclear Safety, N Stanton (ed.), pp. 117-140, Taylor \& Francis, London.

Sterman JD. 2006. "Learning from Evidence in a Complex World." American Journal of Public Health 96:505-514.

Sterman JD. 1994. "Learning in and About Complex Systems.” System Dynamics Review 10:291-330.

Swezey R, R Perez, and J Allen. 1988. "Effects of Instructional Delivery Systems and Training Parameter Manipulations on Electromechanical Maintenance Performance." Human Factors 30:751-762.

Wallace P. 1990. "An Overview of Computer Assisted Instructional Simulation.” In Open Learning and New Technology: Conference Proceedings. R Atkinson and C McBeath (eds.), pp. 314-323. Australian Society for Educational Technology, Washington Chapter, Perth, Australia. 


\section{Appendix A}

\section{TTC Focus Group Discussion}




\section{Appendix A}

\section{TTC Focus Group Discussion}

\section{Task Inventory Development and Validation Discussion Part 1 March 19, 2008}

Gore: Our task was to summarize and document the KSAs required in the CR, which the simulator basically gives the opportunity to practice, demonstrate, teach, etc... From soup to nuts, what must be done in the simulator for training regulators for their jobs - not operators, we understand that!

I started out with NUREG-1122, which lists about 5,000 KSAs, which the license examiners are familiar with because that is the validation and justification for the examination process. I rolled it up to 30,000 or 40,000 feet, into about 20 skills and abilities. Our initial thought was to skip the knowledges - those are taught in systems training. The simulator reinforces them. But we later had suggestions that they are within the scope, so they are now included. I'll come back to them later.

So I came up with roughly a dozen CR tasks, and I did it both by looking at the stem statements - the big top level statements - and by looking at the requirements of the FARs - 10 CFR 45 that says that operator licensing examiners will evaluate these things - (and we have a correlation between those and these) and this is what we came up with. So, for you, feel free to pick - or not - with any of these statements. I would argue that they pretty well envelop your responsibilities.

And we have made a distinction just in the last week, and we have gotten away from the "ability to operate" language that's in NUREG-1122, and said lets make this specific to regulators, so we started off with \#1, "evaluate operator monitoring", etc. ...reading through several of the task statements.

Minsk: So what we're asking you guys to do in this session is to tell us if there is anything we have here that is wrong, and shouldn't be there, or worded incorrectly, or if there is anything that should be added to this list.

Question: We're just looking at this from the perspective of what equipment we're going to have in the simulator? Not if we're evaluating operators, but for our own training? What we'd like to see as a simulator for a new construction plant??

Gore: This is a little different from just thinking ahead towards physical fidelity. This task is somewhat broader...to scope all of the Ks, Ss, As that go on in the CR. This is as a validation, a basis, for the training - how are you going to train, what equipment do you need, what physical fidelity must be required...Did I get that right, Mark?

Miller: Yes. The way you ought to read this is, you're an NRC resident, or inspector, or license examiner - you're in the CR - this is the list of stuff, broadly stated, that you think that you need to know how to do based on inspection procedures and license examining standards. This is the list of stuff that applies to you in the CR - not out in the rest of the plant - it's a list of CR skills for the regulator - and if that's right, or as it's modified or massaged based on your input and other inputs - it's going to become sort of a grading sheet, so that when you look at a simulation, you can say, maybe this kind of a simulator may not allow me to evaluate responses to automatic actuations... 
Gore: Sort of a baseline... Continues sketching through the task list and concepts addressed.

Q: Some residents are encouraged to use the plant computer in the CR? Gore: I would imagine that they probably don't mess with it in the CR. They may go into the Shift Technical Advisor computer.

Discussion followed, of computer resources that can be available to the resident throughout the plant....

Gore: ...continues sketching through the task list through the operations tasks.... That, I would claim, at the 40,000 foot level, pretty well spans what goes on in terms of operations in the CR. Next we get to management...

Gore: Examiners, when they are giving SRO examinations, will evaluate that guy in the position of shift supervisor, or as a potential shift supervisor. So management and leadership of the operating team in the CR is a major activity that needs to be evaluated and potentially to be addressed in simulator training.

Discussion of resident inspector activities evaluating management and leadership in the CR...Well, we do it - management asks for our feedback on that, but we don't have a lot of regulatory footprint, in that we can't issue findings in that area. They certainly have a conduct of ops procedure at most plants. We look at that to see if things happen according to that as far as the management...

Gore: Yeah!...if the balloon goes up...When they get that small break LOCA, does the SS, or the guy who's acting as SS, take charge, get into his emergency plan, and exert command and control?

Additional discussion of management in normal and off-normal situations. Question and comment about connection between verbal communications in the CR, and simulator training. Gore: "Forget about simulator fidelity. This is an attempt to envelope everything that goes on in the CR that's important from a regulatory standpoint. Either to an operator license examiner or to a resident inspector's viewpoint." Additional discussion of the extent of coverage in contrast to having 5000 different statements...Acknowledgement of 10 CFR 45, and the crosswalk to these tasks.

Minsk: Farther on in the analysis there will be an analysis of ...DIF...

Additional extensive discussion of the difficulty of dealing with the huge number of things involved, and the high level of these few tasks - whether everything is covered and the list is comprehensive.

Discussion of resident review of post fire and other safe shutdown procedures to determine both workability of the procedure and ability of the team to accomplish the tasks within time allotments. Where is this covered in the list? Gore: Command \& Control of SS; monitoring and operation of equipment; resolution of issues including license related issues.

Comments on the residents' responsibility to evaluate not only operations, but the design of procedures. "We do all kinds of things in the CR, it's not just watching what the operators do. Review how systems operate, timing between systems, how quickly can certain systems be brought on line..."

Discussion of fidelity impacts on operations. Gore: What other things does it influence - CR turnover? Yes.

Gore: The simulator is a stage where examiners watch CR crews interact, and make career decisions on licensing.

I think this does a pretty decent job of listing KSAs and our job in the CR. However, I think that most of the things on here - evaluate operator performance, evaluate the use of procedures - are mostly learned 
on the job, not in the simulator. Agreement. Gore: You won't ever be allowed to touch the boards. Just evaluate. Discussion of the importance of knowing the operation well enough to think ahead of the operators and know if they are going off in the wrong direction, or are proceeding appropriately, as the basis for evaluation.

Discussion of resident responsibilities during emergencies, gathering information, evaluating, reporting. "It's rare that we are called on to do that, but it's the reason why we have resident inspectors." "When an emergency occurs they are not evaluating. They are actually involved. They are in the thick of things." This discussion eventually led to adding a task to the Ops part of the taxonomy: Provide communications with NRC during declared emergencies.

Discussion of Ops-4 resulting in a consensus to add functionality to the availability and operability determination, and replace safety related systems by plant systems. Additional discussion of whether Evaluate is needed - difference between evaluation of operators and independent determination of status.

Discussion of Lic-1 resulting in a consensus to add other reportable conditions to items that operators must recognize and respond to.

The list does a pretty good job of covering a lot of things that inspectors and evaluators do in the CR, but we don't learn a lot of them in simulator training, and I don't think we should. Minsk: There will be another analysis of what's important in training.

Minsk catalyzed discussion of the information requirements needed for tasks, and how difficult it would be to break that information out. Comments that there are thousands of things to do, just to verify functionality and operability. You would have to walk down the entire panel, switch and valve at a time!

One of the things I gained tremendous value out of the simulator, coming out of college without industrial experience, I learned simple basic navigation through the EOPs, simulating events and operation of the reactor. I don't necessarily learn how to inspect and evaluate the licensees, even though eventually I need to get to that point as an inspector. I don't learn all that in the simulator. Comments on the importance of seeing these events in the simulator due to the paucity of events in the plant.

Subsequent discussion of the question of information needed and how difficult it would be to break that information out. That would be a fairly big, hefty job!

Facetious discussion in closing of the importance of evaluation and sampling of CR popcorn. The problem is that these simulators don't have it. Instructor: We simulate it! Response: That's the problem!

\section{Task Inventory Development and Validation Discussion Part 2 March 19, 2008}

Discussion of evaluation of operator response to annunciator initiation (Ops-8). Focus on independent identification of proper response and use of this in the evaluation process. Eventual subsequent agreement on insertion of in accordance with guidelines in administrative procedures to replace including silencing of distracting alarms, removal from service, and restoration.

Discussion of the use of "in accordance with regulatory guidance" related to Davis Besse tornado where the licensee notified NRC that they were going to violate their technical specifications (TS) by exceeding 
cooldown rates, according to the $50.54 \mathrm{X}$ policy, and the query to the resident whether he agreed with that decision. Eventual agreement that this was, in fact, part of regulatory policy.

Discussion of Ops-10 and whether the resident can use the plant computer himself (in some places he can, some not). Agreement that the statement envelopes responsibility.

Discussion of Ops-11 evaluating shift turnover. Assertion that that this would lead to need for a more detailed simulator, in order to do a detailed panel walkdown. Gore question if this could be done with flat panels showing pictures and replicas. Answer - it would need the same information and format as the plant. Incomplete discussion of the meaning of "full scope" - whether it means (Gore) that all aspects of operation are addressed or whether it means that all hardware is faithfully replicated. Instructor commented that he feels that the TTC simulators are full scope, or near full scope, even though they replicate plants that no longer exist. Minsk redirected the discussion before agreement was reached on a definition of full scope.

Minsk: Comment that risk concepts needed to be inserted. Agreement, but acknowledgement that NUREG-1122 was done in 1984, so risk was not yet included in the concepts. Moved on with a thought that it might be addressed later in the K's. Eventual development of a new Mgmt-5 Evaluate the implementation of administrative procedures associated with risk assessment activities.

Discussion of Mgmt-1 and agreement that evaluating the management and leadership of the CR team was too sweeping a statement, and that it should be replaced in the statement. Extensive discussion of SRO grading \& statement that SROS are graded against the competencies required by NUREG-1021, ES-303. Eventual recommendation of Verify and report CR command and control.

Brief read-through, with minimal discussion, of the remaining Mgmt tasks.

Brief read-through with minimal discussion of the Lic tasks. Objection to use of the word timeclock in Lic-2. Suggestion to use LCO action statements and it's dismissal.

Inconclusive suggestion of "reporting criteria'. Eventual suggestion of "allowed outage time".

Brief read-through with minimal discussion of the Ks. Comment that risk concepts might be added to K-2. Question about including security items into the task list. Eventual decision not to add security.

\section{Discussions with TTC Instructors on DIF Analysis of Task Inventory March 19, 2008}

Minsk: Task Inventory will be subjected to further analysis - one is a modified training analysis called DIF analysis to prioritize for training, and also an analysis to determine of what simulator fidelity level is necessary to teach these tasks. Now we'll talk about DIF Analysis. Want feedback from instructors if anything else we should do, or if it seems like we are doing anything inappropriate.

Traditional DIF= Difficulty of performance, Importance to safety, and Frequency of performance.

Modifications: How difficult it is to learn the task; How important is it to train on a simulator to learn the task. 


\section{References: IAEA Systematic Approach to Training}

Instructor: We bring people here and give them an opportunity to do things, but they will never do it. They will only observe other people doing that in the field! Is it difficulty of observation, or difficulty of performance?

Gore: Is there a correlation between difficulty of performance of a task and the difficulty of observing and evaluating whether it's done right?

That's exactly what I was saying. - General agreement that you have to know how to do it to know how to evaluate how well it is done.

Discussion of a surveillance procedure - all you can see is if he is following procedure and administrative guides if evaluator does not know the facility and operation...

Gore: You need to know how to do it, to really evaluate. You must know what the operator is trying to do, think ahead, and be ahead of him in the evolution and in expectation of results. That's what we mean by evaluate!

Some of that preparation is incumbent on the inspector, to go do his homework and look at this stuff. Hopefully he has been to the TTC and had a chance to play with these systems. To understand how the system operates, and how it's put on line, but not the details of power supplies and interlocks and things like that. He's not going to be a licensed operator. He's going to be an observer of licensed operators' activities.

Gore: Comment on initial plan to ignore knowledges until directed to include them. They are trained in systems training. The simulator gives the opportunity to apply, integrate, and synthesize.

Q: Is the DIF analysis targeted at students coming out of college, or at experienced people? There will be people with widely different backgrounds. Suggest bounding it by examiners, residents, and by bounding experience levels (high, low).

Gore: I suggest bounding it by the basic difficulty of the concept. They are not going to be able to hire ex-Navy nukes anymore.

Discussion of refresher course - is it mostly aimed at experienced people? What is the amount of meaningful experience gained in 3 years since initial training? Comment: They may not have done ANYTHING relevant at NRC during those 3 years!!!

Minsk: Is the intent of refresher training to address experienced folks? NO! Refresher training purpose is to get them back in school once in every three years. If they want to maintain certification, every three years they have to take the courses. I would make absolutely zero assumptions other than that it's been 3 years since they took the course! Additional discussion of refresher training requirements, and number of years before refresher training on a given type of plant if they are certified on several types of plants.

Our targeted audience should be those who would benefit from using the simulator. - not the ones who just have to come back every 3 years, because they don't need the simulator! Don't dumb it down for the novice!

Will you look at industry requirements for what they consider infrequent operations on your analysis of frequency? Startup, shutdown... Now they may only do a startup every year and a half. 
When I was on the AIP at Zion, the crew who had to do the shutdown due to the inoperable spray pump had never done a shutdown on the plant!

Gore: Pointed out the 10CFR45 requirement for pre-startup operations through power operations. Comment: That's for initial examinations. These guys had been on shift for 5 or 6 years and never done a shutdown on the actual plant!

General discussion of fidelity requirements for these things. Diffuse comments - no conclusions.

Gore: What does NRC have to be able to teach, with adequate fidelity? Is fidelity more important for the guy just out of college, who hasn't been on the boards, doesn't understand nuclear power, or is it more important to have that fidelity for the guys

who are coming in for refresher training, requal? Ans: We don't have full fidelity now, nor do we teach to that level. We have fidelity good enough to do what we have to do, now.

Could we dispense with the old simulators in this building, and go to all flat screens and model this without having any hardware, joy sticks, at all. One reaction - that would be insanity! Another: If, in fact, the CRs go to digital, and flat panels eventually, if the analog controllers are modeled on the simulator, yeah, you might be able to model it on a flat screen.

After years on an analog plant you know where you need to be - where to look and stand - if there's something going on in the $\mathrm{CR}$ - because I can see the annunciators. I know where the reactor control station is, I know where the turbine control station is. I can monitor that stuff. It's a different layout, it's a different physicality, different alarm systems... That's the fidelity that needs to be there. How does the crew work together and respond together? Where do I need to be looking to see what's going on?

The only difference between the old and new plants is the man-machine interface (MMI). It doesn't even matter if it has a digital RPS or not. The thing that will need to be different for the new plants is that MMI. The systems, the plant operates the same. The key is to figure out how do we help people along with this MMI? And how the CR will function and look in that new environment. We don't even know at this point what the MMI will look like.

Minsk: Comments on the envelope of what's possible based on NRC generic guidance on HSI, and info on Lungmen, and other proposals. All have proposed large screens in front, a lot of flat screens for individual operators and SS, all have some back-up hard control panels. We have seen that at least a couple of them have mimic displays on the operators' screens that you can navigate to get to systems level and get to components, and can implement controls on those mimics. Some of them (not Lungmen yet) have procedures on computers.

That's the MMI I was talking about.

Gore: One of the things that I jumped on early in this was, regarding fidelity, are we talking about menu fidelity, algorithm search fidelity, but in this project we are assuming that whatever comes in for CRs will be available to NRC.

Minsk: We realize that the simulators here are pretty dissimilar in fidelity from what's actually in the field. In the initial survey we wanted to get a sense from folks of, does that have an impact on your actually performing your job. Just from glancing at the surveys that were done today, the responses were $1 \mathrm{~s}$ and $2 \mathrm{~s}$, mostly $1 \mathrm{~s}$ - it doesn't have any impact! So, for us that may open the door for varying levels of fidelity and something that should be a lot easier to replicate, because we'll be replicating computer screens with computer screens, probably. 
You only have 2 weeks for initial simulator training, 1 week for refresher training. There are only so many things you can get to. Agreement.

Bates: The learning objectives for simulator training--- where did they come from? Laughter and hoots! They sat down and wrote them. The original simulator class used to be this same class!

Miller: What we heard in both classes from the students, was a unanimity of opinion it seemed - or at least a plurality - that they don't learn stuff they need to be a good inspector here. They take the stuff they learn here, and they add a lot of OJT to it, and then they go out and inspect... (Comm.) We're not teaching them how to inspect. And that's what kind of begs the question for me - how did we decide what we're trying to achieve? We're trying to teach them how a real plant works.

Instructor: We don't teach them evaluation. We try to show them an example of how something is done, if they were an operator. - and that's what they should be watching for the operators to do. When I first started here, the goal was to teach $80 \%$ of the SRO knowledges to students. But back then $80 \%$ of the knowledge was less. 20 years ago when I first went through the SRO program, it was a 3 month program. How much can you learn in 3 months? So we were trying to teach an $80 \%$ knowledge level which now is more like an $8 \%$ level!

I think Mark has brought up a million dollar question...I've only been here 3 years, but I've never been able to figure out, and no one has ever told me, what the target audience was (we're talking SAT).

To be quite honest, there were a lot of old operator guys here, and they weren't a lot of old regulator guys, so the course was designed as a bunch of old operators.

The target has been to teach operations, not how to inspect.

Minsk: Tell me if this is fair. I think that your target might be to teach them to understand operations well enough so that they can evaluate it. Approbation. So that leaves an open question - do these guys need enough fidelity to interact with the systems the same way an operator does, in order to learn that sufficiently?

I think as a minimum you need enough fidelity to be able to train them on the use of the off normal and emergency operating procedures, and that encompasses both the resident inspectors and the examiners. So they can work through the procedures like they would be done in the CR, so they understand how that would work. To build up to that, what we do is we start them off with simple operations, getting them used to how the plant works (starting up, maneuvering the plant at power), and figuring out where the controls are. And then we start doing transients and the EOPS, so they can work as the operators do, and see the difficulties, nuances that are involved in that. And then when they go to the site, evaluating drills, evaluating requal, then they already have a good idea in their mind, once they've done it themselves. So then they go out and build on that.

And then there's another class that examiners come here for a week to do, where they actually come in and write an operating exam and then use our simulator to give the instructors an exam. So that simulator downstairs works well enough to do that.

General discussion of regulator knowledge and inspector credibility when interacting with facility personnel.

Discussion of inspector time in CR (small) vs. time in plant (most). 
Gore: You've been teaching them to operate. Do they have to actually operate - turn things on? Or could they be trained by seeing presentations of stuff operating?

I think that's a very valid point. We've talked about this. Do we ever show them what a real operating crew is supposed to look like? No. Do we ever demonstrate to them what they should? No. Because their job is going to be is standing back here with their clipboard, trying to be nobody, and just watching. They are not going to touch anything.

Instructor: Again, that's not what we try to teach them. As of now, that's not what we try to teach them.

My answer to that would be - my personal opinion - is, there is a difference between watching, and doing. One of the things that I like to reinforce to the folks that come through here for refresher training, who maybe haven't done it in a while is: Those of us who never do, start to make assumptions as to how easy it is. We tend to get in a different realm. But when they come in and they have to open up a procedure and follow it, and the procedure isn't well written, and they've got to turn the switch...OK, it really drives ... Most of us learn well from failure. And watching somebody else fail is not the same thing as "Oh, all the rods fell in, and it shouldn't have" and "Oh, it's not as easy as it looks!" There's nuances, there's things you have to pay attention to - that things just don't happen... and I think that there is a tremendous amount of value to somebody who has never done it. At least being exposed to it, and having to do it... if only that one time. Different target population - if we were hiring all ex-navy nuclear operators, if we were hiring all ex-utility operators, and we just wanted to show them on video.....

That's a good point! There is a balance between doing, and being humiliated enough to know that that's not easy to answer...

There is a benefit. Is it required? No. Does it help? I think it does. That would be my personal opinion...

Instructor: They did a survey a long time ago when they were planning to get rid of the $\mathrm{B} \& \mathrm{~W}$ and $\mathrm{CE}$ simulators... So they went out to all the inspectors and examiners and everybody else at the time... and said "Hey, are these things worth keeping, or not?" NRC wanted to move everything. If we would move to HQ, the big question was should we take the W and GE simulators, or do we take all four. A lot of the people fed back that there are enough differences that it is important enough to keep the other two simulators.

Gore: With regard to the question that I asked, I learn by doing. I can be told a zillion times, where to go what to do, what to look for... and not find it to Wal-Mart, for pete's sake. Whereas if I drive there or walk there I have a chance of remembering it. And that's certainly true for operating any piece of equipment...

You're talking about learning styles. We all have different learning styles....It's a balance...

One of the perfect things that we do in the training, almost routinely, is at some point in the training we say, "Hey! Before you go trip these bistables, you should check and make sure that it's not going to cause something bad to happen." We say it. Almost invariably, whoever the trainees are, the first opportunity they get they'll go trip a bistable, and put all the rods on the bottom. Thereafter, we rarely can get them to do it again...

I've even gotten seasoned guys to bite on that one.

Yes you can. 
We also have anecdotal evidence from people going through our systems and transient classes, who had failed one or both, who were close enough and went through the simulator, "After I saw it and did it, it made more sense to me." And they do fine on the retakes because they have the learning style that it made more sense after seeing it and doing it, than after having it explained to me.

Comment on the necessity of having analytical fidelity of following basic physical laws.

Minsk: We are going in with the assumption that thermohydraulic simulators will be full fidelity.

Gore: Is it agreed by everybody here that whatever simulator we get - it's got to be operable by the trainees. Yes. But, it might be acceptable - it's at least a negotiable, arguable principle whether it's operable by hardware and switches, or by putting fingers on a touch screen or by manipulating it by some other computer digital-related HSI...

Minsk: So, for example, if you have something that requires the functionality of a switch, it's generally implemented with a switch control. That switch doesn't have to be a physical toggle, it can be a flat display, or whatever,.... as long as it has the same effect on operations.

Discussion of CR layout and crew interactions, and different operating stations. The trainees have to see the whole interaction of the CR, and operation of the crew. But that can be a bunch of flat panels...

Soft controls should have a sufficient level of nuance to represent that actual physical switch - instead of just having a button that you can push. If you're watching it, you need to have enough understanding of whatever representation you're using in the simulator, that you can see that the operator in a real plant is operating that switch correctly. There is some value to actually seeing how that switch operates.

Discussion of cost - suggestion that thermohydraulic fidelity may be the biggest cost, whereas physical fidelity may not be that much additional. Also questions of space needed. Comments on hardware problems with present $\mathrm{B} \& \mathrm{~W}$ and $\mathrm{CE}$ simulators.

Miller: Question about a work group - what do they want out of simulator training? Answer addressed a Steve Shaw...only addressing initial training...Miller: What do they want out of initial training? Ans: It only addressed changing the qualification card looks. What we're doing here seems great.

Minsk: One thing that I heard today in both the student sessions - they felt that they need some knowledge of how to do the operations to evaluate how the operators do it. That would support the statement that we do need simulator training, because you can't do it in the field.

Minsk: Disc'n of conversation with head of FAA training program for FAA inspectors. They do refresher training every year. They do it very differently - it is farmed out to flight schools and universities with flight programs. The aviation safety inspectors are required to be pilots. They must have so many hours - not a whole lot - but they all have experience being pilots. The FAA wants to make sure that their guys are at (maintaining) standards, since they don't get to fly any more unless they do it on their own dime. Also, there are many changes in aviation technology, with glass cockpits, and they need to get their inspectors exposed to the technology on simulators since they are not flying. Also, they are certified on a type, say a jet, and they can do inspections on all kinds of different jets and different cockpits, not just what they were trained on. So they are mainly evaluating - are they following procedures correctly? 
Comments on how much the inspectors may not pick up on as a result of inadequacies in their training. Rather diffuse discussion of training people out of college using simulators - both for flight and nuclear operations, if you have enough time...

Minsk: Would you say that you guys have an objective, maybe not spoken, to train these guys enough so that they understand and appreciate plant specific differences - not so much that they could understand enough about how things work...I'm not saying that well...interrupted...

We give them one example of how a plant works. Downstairs we use a 4-loop Westinghouse plant there are 2-loop Ws and 3-loop Ws that work totally different from what the 4-loop plant does. The injection systems and the ECCS systems are totally different. But we give them a base - this is how this plant works through the procedures. The procedures on the other plants are going to be different. Other low pressure injection points. They are going to be different.

If I could make an analogy... You said that you were in a flight simulator, and with an instructor there you took off, you flew, and you landed it... Do you think that's enough for you to be qualified? Minsk: No.

That's about what we're doing with people, too... We're bringing them into the simulator, we're doing startup, we're getting to operate, we're shutting it down, and then we're doing some emergency stuff. So, that's what we're working with, to give the basis for people, so they can go out...

But that same guy then goes to a bunch of other courses on conducting inspections, on field techniques... How do you use this technology at the region to zero in on the stuff that NRC's concerned about at the operating plants?

If the plant has a good senior, hopefully, once they get out there...

This is just one piece of the overall...one block of a big chunk...This are a couple of signatures on a qual card.

As a resident, I used to enjoy coming back to take one of the courses here, that we call the $704 \ldots$...because you can be out at the site, and often you get the feeling that you're sitting on a time bomb there... and if there is an accident, you really haven't looked at the EOPs real recently.... and the bulk of your stuff is just normal operations and surveillances and maintenance...but nothing real bad, fortunately, ever goes on. So you feel in the back of your mind that you don't really remember how that EOP situation works out.

So when you can take a week off and come here and in a week's time run through all this stuff, imperfect as it may be, and limited as it may be, with the capabilities of the simulator, and not being able to take it all the way through, but still... It gives you a refresh view of the procedures that are involved, of the concepts; it reinforces some stuff that the cobwebs have begun to sink in on so it's a tremendous tool!

Discussion of limitations on time and equipment - what we've got is what we've got...this is a suboptimal situation, there are some discontinuities...we have limited control...

What we're trying to do is SAT, so we're going to do this analysis. You make your formula right, and make your weighting factors right... We'll figure out those tasks that we can do in two weeks that are the most important.

Discussion of time limitations, requests from the program office. Comments on evolutionary changes expected as plants hire different vendors to do their life extension and CR upgrades. Comments on 
testing of upgrades (digital sequencers) that didn't work as expected when subjected to the NRC required testing program. (Blocking relays didn't drop if get an actuation during testing, but rather they sealed in!)

Minsk: So, for those of you who haven't gone through the task inventory, if you have time, we'd very much appreciate it if you do. We can talk about it more tomorrow. We didn't really talk about the details of that today.

Discussion concludes.

\section{TTC Focus Group Discussion Thursday March 20, 08 AM -Notes taken by B.F. Gore,}

\section{PWR Class, Instructors Phil Gephart and Bobby Eaton}

Focus Question \#1: What are the impacts of differences between TTC simulators and your work assignment CR?

A\#1: (Entered out of college) Minimal impact - focus is on procedural requirements

A\#2: I'll take it a step further, they don't even have to be vendor specific! I'm a resident - It'll be all over when I get to the CR. (I've been licensed on both PWR and BWR.)

A\#3: Navy PWR background. Vendor training refreshes us on details. This training has nothing to do with our day-to-day jobs (EOP training).

A\#1: Channel failures were more applicable to our daily jobs.

A\#4: (Resident) Your satisfaction from training depends on your previous experience. I was fresh out of college 17 years ago. TTC training brought it all together for me. But, you need plant specific training. I have to learn my plant.

A\#2: Here's a rule of thumb. It takes 3 years for an engineer to learn his job to where he is adding value instead of just using resources.

A\#1: TTC shortens the up-to-speed time. He tried to say something about negative training, but was cut off...

Instructor Eaton: We only have 7 weeks, not 2 or 3 years!!!

Followed by considerable discussion that in that short a time, students learn concepts for subsequent follow-up and refinement, not specifics of operation.

A\#4: There is some importance to learning vendor specific details - but we do have on-site resources. There is value to putting your hands on the switches. A touch screen would be acceptable.

A\#5: There is better retention than if you watch!

Minsk: How about virtual reality? 
A\#5: That's a broad question to ask the industry. If the controllers modeled on the computer screen don't look identical, that's not a big deal.

A\#1: (finally gets his comment on negative training in) If moving from a BWR to a PWR, that would be negative training. The mimic of a swich should have the same information as a real switch.

Miller: The old simulators will still be here. We can train on them to show "real switches".

(not sure who): Davis Besse has screens as part of their SAT based training. Afterwards they go into the simulator. They just don't have enough simulator time!

Gephart: Lets talk about crew interactions. What about different size crews? 2 vs 4 ?

A\#1: It would benefit training to be as realistic as possible. It would benefit the agency to get them out to plants as soon as possible.

Minsk: I feel a concensus. There needs to be interactions with the plant. We don't necessarily have to have the same controls - physical fidelity.

A\#5: All Westinghouse plants have the borate switch. Training should have the switch.

A\#1: A picture would be OK. Even a screen reversed left to right wouldn't matter. That situation actually exists in some CRs!

End of detailed notes -Focus Group discussion at TTC March 20, 2008

\section{Thurs AM Transcribed Disscussion - Recording Starts after the Discussion \& Hand Notes Started. Topic Addresses Recurring Training}

--------I'm doing this again - and again- and so for me there's not a lot of value.

Gore: - Which is why examiners and NRC likes to hire ex-navy nukes. 'Cause they already know the boards and the TSs and procedures...

Well - we don't have TS in the Navy.

We don't even have Boron in the navy!

I would lean more towards ..unintelligible...

I came out of the navy, so I had all the plant knowledge - how they work background - for navy PWRs. I find going to the vendor specific training worthwhile because it refreshes me on setpoints, interlocks, intersystem interactions, for the plant where you are. I agree with Mike here, that this training has nothing to do with our day-to-day jobs, day-in, day-out.

Gore You're a resident? Examiner? 
Well, both. I'm in construction now, so I'm neither. But I was the Sr Res at Watts Bar until this last June. But I also agree with Mike. I come to the refresher as an examiner every 2 yrs and as resident every 3 yrs, and for me its just a break from routine. Do I feel I need to come to referesher every 3 yrs? No.

But they make us come anyway.

Well, that's above us. It's a policy...

So there's something else - it's a major change in policy.

....and I still enjoy it. It's got some worth, to some extent, because it gets you back on the panels, lets you manipulate the switches, lets you see the plant's performance. I think last week probably was a little closer applicable to our daily job than this week. The technology, the TS, the day to day operations which is what they do every day. But this week, this EOP portion...

Is what you hope that you never do.

..is what we hope that we never do. But if we do...

We have to be ready!!!

**** skipping discussion - specific, extracted quotes and summaries follow

Resident: Your satisfaction (with the training) is highly dependent on the skill sets you had before the training. When I was going through boiler training, it was at Black Fox, but I was stationed at Dresden. So there were tremendous amounts of differences. But you can deal with the differences. You spend day-in, day-out at the plant. You learn the plant specific differences. So the differences of the simulator (from the plant) do not bother me. When I go back I still have to learn the plant. Whatever we learn here just sort of helps you coming out of it. I felt I was helped tremendously with my job. Coming out of TTC doesn't mean that you are fully experienced and fully qualified. But at least it gives you the basic skill sets that you can take and learn, and build your experiences.

Gore: So the differences have minimal impacts because you have the concepts and can apply them to the specifics, as opposed to being an operator where there is a big emphasis on "Don't give them negative training."

\section{$* * *$}

Instructor: All we have here is 7 to $10-12$ weeks, whereas you're talking a three year program! We just don't have the time here. We just begin to do things, and then it's up to the individual to be able to get out there and work on their own....

Gore: Let me come back to your comment. I like the one about, "There is some value to putting your hands on the switches." [laughter] But let's take that a jump shift, How about, if instead of having the hard panel and the switches, you had a computer with a screen and a touch screen, vs that.

You know, I think that would be acceptable also. Personally, I would. And I think that's what the CRs will be moving to in the future, and that's what the operators will be using. 
Minsk: Does it matter if a controller is functionally the same, but looks different from the actual plant? (lots of chatter and interruptions...)

I don't think that matters, if there are different style switches or controllers. Agreeing comments... discussion of tactile vs conceptual learning...

That's probably very dependent on the person's experience. You know, I can look at that and I can equate that with switches I have seen and manipulated, so for me its no big deal. But for a person coming out of college, they may not equate that to a physical swith....interruptions and discussions about experiences with computer games, etc...

Bates: Suppose we can replicate a switch exactly on a CRT screen, so its not 3-dimensional, but just flat. Do you feel, since you don't actually operate, but are observing the operation of others in the plant, that its necessary for you to have the tactile feedback of what it is to take the control switch to pull-to-lock, since you can't do that on a CRT screen, but you could accomplish it on a touch screen by just touching the switch in the appropriate position. Is it most important to learn that that function is there, or to gain the tactile feedback of actually moving it?

I think it's important to know that the function is there, and I'm used to doing it like that. The next generation, they're going to be used to doing it like this. Additional discussion of future CRs vs this generation of CRs....

Without predicting future CRs, as long as the mimic of the switch (however you do it) has the same options as the real switch has, and performs the same functions, I don't think it matters whether it's a touch screen or an actual switch.

Discussion of physical mockups, and other possibilities for training the physical concepts... Also, an instructor noted that the old simulators will still be at TTC, and can also be used for examples of physical switches in training.

$* * *$

Gore: You set us up for this question very nicely when you said the training here gives you the concepts. And this certainly, to me, gives you the concepts. And whether it's one of those controllers that's in here, or a touch screen, or whatever, as you said - you picked that up earlier - but I didn't want to put that in anybody's mouth. We're here to get your ideas.

Discussions of similar training concepts already employed at Davis Besse due to limited simulator availability. Computer training outside of simulator as part of their SAT-based program - then class goes into the simulator for additional training.

$* * *$

Minsk: - attempting a high level summary: I think we have a concensus that there needs to be some interactivity with the plant. You are able to input some type of control interactions as in a similar plant. But it doesn't necessarily have to have the same controls and displays. Is that true?

Yes. It's still a learning process. Additional discussion of various representations of the borate switch at Westinghouse plants followed. 
What if everything was reversed - left to right? Doesn't matter?

Quad Cities and some of the other Excellon units have that right now!

Discussion of mimics and their importance.

Minsk: I'm not sure we've converged, and that's fine. I hear that sometimes it matters that we have something that looks like the actual plant, sometimes it doesn't.

I'm thinking that the big delta will be between the experienced people and the inexperienced people, whether it matters or not. For me it doesn't matter, 'cause I've been there, done that. But for the guys fresh out of college it may matter. I don't know. And I don't think that this group can answer that.

Discussion of DVDs with simulator capabilities and other advanced capabilities for exam writing - doing requal in home office - virtual simulators with avitars....Miller:--- that's way over the horizon stuff...

Gore: Backing up again to 30,000 feet, 7 weeks at TTC. There is no way that you can teach anything significantly more than concepts. This is a way of teaching concepts, and the people in the room can take those concepts and translate them to hard controllers - if and when they need to.

Miller: brief discussion of short courses for various groups, and what to cover, and enabling and terminal objectives - what do you want these people to be able to do?

$* * *$

Miller: We have to be able to articulate what we accomplish in this course.

$* * *$

Miller: All right! We appreciate your time! 
Appendix B

\section{DIF Analysis Rating Scales and Results}




\section{Appendix B}

\section{DIF Analysis Rating Scales and Results}

\section{B.1 Rating Scales}

Table B.1. DIF Criteria for Rating the Task/Inventory Items

\begin{tabular}{|c|c|c|}
\hline \multirow[b]{2}{*}{ DIF Dimension } & \multicolumn{2}{|c|}{ Scale Definitions } \\
\hline & RI & OLE \\
\hline $\begin{array}{l}\text { Difficulty of task performance in } \\
\text { current job assignment } \\
\text { Knowledge items: Difficulty of } \\
\text { recalling and applying the } \\
\text { knowledge in current job } \\
\text { assignment }\end{array}$ & $\begin{array}{l}1=\text { Very Low } \\
\text { to } \\
5=\text { Very High }\end{array}$ & $\begin{array}{l}\begin{array}{l}1=\text { Very Low } \\
\text { to }\end{array} \\
5=\text { Very High }\end{array}$ \\
\hline $\begin{array}{l}\text { Importance of task to safety } \\
\text { Knowledge items: Importance of } \\
\text { knowledge to safety }\end{array}$ & $\begin{array}{l}\begin{array}{l}1=\text { Very Low } \\
\text { to } \\
5=\text { Very High }\end{array}\end{array}$ & $\begin{array}{l}\begin{array}{l}1=\text { Very Low } \\
\text { to } \\
5=\text { Very High }\end{array}\end{array}$ \\
\hline $\begin{array}{l}\text { Maximum frequency of } \\
\text { performing task in current job } \\
\text { assignment } \\
\text { Knowledge items: Maximum } \\
\text { frequency knowledge is used in } \\
\text { current job assignment }\end{array}$ & $\begin{array}{l}1=\text { Once a year or less } \\
2=\text { Multiple times a year } \\
3=\text { Multiple times a month } \\
4=\text { Multiple times a week } \\
5=\text { Multiple times a day }\end{array}$ & $\begin{array}{l}1=\text { Seldom if ever evaluated } \\
2=\text { Evaluated in some but not all } \\
\text { simulator exams } \\
3=\text { Evaluated at least once in } \\
\text { every simulator exam } \\
4=\text { Evaluated in most scenarios } \\
\text { of every exam } \\
5=\text { Evaluated in all scenarios of } \\
\text { every exam }\end{array}$ \\
\hline
\end{tabular}


Appendix C

Task/KSA Types and Levels Rating 


\section{Appendix C}

\section{Task/KSA Types and Levels Rating}

An expert panel applied the following rating protocol to characterize the level of KSA needed for each type of KSA for each item in the Task/KSA Inventory. The results of this rating process were used in assessing physical fidelity requirements for the different items and, overall, for the RI and OLE positions.

\section{C.1 Rating Scales}

\section{C.1.1 Knowledge}

\section{C.1.1.1 Factual Knowledge}

Facts - basic, accepted information elements or details; terminology; definitions. Facts differ from concepts in that they are 'elemental' and essentially stand on their own. Example facts: the name of the street you live on; regulations that apply to operating a commercial power reactor in the US. Facts can be critical to performance of a task, but it may not be necessary to recall them if they can be referenced/looked up during the task without impacting the performance. Also, recalling a single fact may be essential to performing one task, while recollection of many facts may not be essential, but helpful, for another.

Select the right-most statement that is true for the task being rated.

\begin{tabular}{|l|l|l|l|l|l|}
\hline $\begin{array}{l}\text { Internal } \\
\text { knowledge } \\
\text { of facts }\end{array}$ & $\begin{array}{l}\text { No recollection or } \\
\text { recognition of } \\
\text { facts needed for } \\
\text { successful } \\
\text { performance of } \\
\text { task. }\end{array}$ & $\begin{array}{l}\text { Recollection or } \\
\text { recognition of } \\
\text { facts is helpful, } \\
\text { but not essential, } \\
\text { for successful } \\
\text { performance. }\end{array}$ & $\begin{array}{l}\text { Essential to } \\
\text { recognize that } \\
\text { observed } \\
\text { information } \\
\text { accurately } \\
\text { represents } \\
\text { something known } \\
\text { to be true. }\end{array}$ & $\begin{array}{l}\text { Essential to } \\
\text { recall basic } \\
\text { facts from } \\
\text { memory. }\end{array}$ & $\begin{array}{l}\text { Essential to recall } \\
\text { or recognize } \\
\text { basic facts from } \\
\text { memory quickly. }\end{array}$ \\
& & & & \\
\end{tabular}

Select the right-most statement that is true for the task being rated.

\begin{tabular}{|l|l|l|l|l|l|}
\hline $\begin{array}{l}\text { External } \\
\text { information } \\
\text { about facts }\end{array}$ & $\begin{array}{l}\text { No external } \\
\text { information about } \\
\text { facts needed for } \\
\text { successful } \\
\text { performance of } \\
\text { task. }\end{array}$ & $\begin{array}{l}\text { External } \\
\text { information } \\
\text { about facts is } \\
\text { helpful, but not } \\
\text { essential, for } \\
\text { successful } \\
\text { performance. }\end{array}$ & $\begin{array}{l}\text { Essential to use } \\
\text { external } \\
\text { information to } \\
\text { verify that } \\
\text { observed } \\
\text { information } \\
\text { accurately } \\
\text { represents } \\
\text { something known } \\
\text { to be true. }\end{array}$ & $\begin{array}{l}\text { Essential to use } \\
\text { external } \\
\text { resources to } \\
\text { access factual } \\
\text { information. }\end{array}$ & $\begin{array}{l}\text { Essential to use } \\
\text { external } \\
\text { resources to } \\
\text { quickly access }\end{array}$ \\
$\begin{array}{lll}\text { factual } \\
\text { information or } \\
\text { verify observed } \\
\text { information. }\end{array}$ \\
\end{tabular}




\section{C.1.1.2 Conceptual Knowledge}

Concepts - interrelationships among elements; structure; classification; categories; generalizations; theories; models. Level of comprehension of a concept can be exemplified by the complexity with which it is employed by the person using the concept. The level comprehension necessary for successful performance of different tasks can vary across different concepts.

- Understand. A fundamental level of comprehension is shown by the ability to explain, interpret, rephrase or summarize the concept or make comparisons or classifications using the concept.

- Analyze. A higher level of comprehension enables one to draw conclusions about a state of affairs using it, decompose something into its parts or functions using it, or integrate parts or functions into a whole using it.

- Evaluate. A still higher level of understanding of a concept allows one to make good judgments about the truth, quality, or validity of something or a state of affairs.

- Create. The highest level of understanding allows one to create new concepts based on the concept that allows one to explain or predict something or a state of affairs better than has been done before.

For each task item and each knowledge item, select the right-most level of comprehension required for successful performance of the task.

\begin{tabular}{|l|l|l|l|l|l|}
\hline & None & Understand & Analyze & Evaluate & Create \\
\hline K-1 & & & & & \\
\hline K-2 & & & & & \\
\hline K-3 & & & & & \\
\hline K-6 & & & & & \\
\hline K-7 & & & & & \\
\hline
\end{tabular}

(K-1) Knowledge of system function, design, and operation.

(K-2) Knowledge of the components in the system and the effects a malfunction of system component(s) would have on system performance, operation of the system, and of the consequences (risk) associated with the malfunction.

(K-3) Knowledge of the physical connections and cause-effect relations between the system and other systems, including knowledge of the effect that loss or malfunction of the system would have on other systems.

(K-6) Knowledge of the physical, chemical, thermal, and electrical properties and processes of the plant and their impact on plant behavior.

(K-7) Knowledge of reactivity effects associated with various plant evolutions and the impact the effects have on plant operations.

Procedural Knowledge 


\section{C.1.1.4 Procedural Knowledge}

Procedural knowledge of how to do something; methods; techniques; knowledge of criteria for selecting procedures. Procedural knowledge can often be tacit or not easily articulated.

For each task item, select the right-most level of comprehension required for successful performance of the task. Do the same for K-4 \& K-5.

\begin{tabular}{|l|l|l|l|l|l|l|}
\hline $\begin{array}{l}\text { Procedural } \\
\text { knowledge }\end{array}$ & $\begin{array}{l}\text { No need to } \\
\text { know }\end{array}$ & $\begin{array}{l}\text { Only need to } \\
\text { know the } \\
\text { purpose of } \\
\text { the thing } \\
\text { being done }\end{array}$ & $\begin{array}{l}\text { Need to } \\
\text { know how it } \\
\text { is done, but } \\
\text { not how to } \\
\text { execute it. }\end{array}$ & $\begin{array}{l}\text { Need to } \\
\text { know how to } \\
\text { execute it, } \\
\text { but effortful, } \\
\text { awkward, or } \\
\text { slow. }\end{array}$ & $\begin{array}{l}\text { Need } \\
\text { fluency. }\end{array}$ & $\begin{array}{l}\text { Need to } \\
\text { know how to } \\
\text { teach fluency } \\
\text { well. }\end{array}$ \\
\hline
\end{tabular}

(K-4) Knowledge of plant procedures, including administrative, normal, abnormal and emergency procedures, and the site Emergency Plan.

(K-5) Knowledge of Technical Specifications and other regulatory requirements for operation and maintenance of the plant.

\section{C.1.2 Psychomotor Skills}

\section{C.1.2.1 Psychomotor Skill Types}

- Motor skill (e.g., movements of hand/fingers) - involves voluntary movement to complete a task

- Visual skill - includes visual memory (ability to recall visual information, including objects and scenes; temporal and spatial aspects), visual analysis (ability to visually match, discriminate, or identify), visualization ('virtual' manipulation of a physical object in one's thoughts)

- Auditory skill - includes auditory memory, auditory analysis (as above with visual skill)

- Tactile skill - includes tactile memory, tactile analysis (as above with visual skill)

\section{C.1.2.2 Psychomotor Skill Levels}

- Familiarity - knowledge of how to carry out the skill, but cannot execute skill reliably

- Controlled - can execute skill reliably but requires attention to execution; can be slow, deliberate, unpracticed

- Automatic - can execute skill reliably with little attention to execution; quick, fluent, efficient, highly practiced

\begin{tabular}{|l|l|l|l|l|}
\hline $\begin{array}{c}\text { Psychomotor } \\
\text { Skill Type }\end{array}$ & Not needed & Familiarity needed & Controlled needed & Automaticity needed \\
\hline Motor skill & & & & \\
\hline Visual skill & & & & \\
\hline Auditory skill & & & & \\
\hline Tactile skill & & & & \\
\hline
\end{tabular}




\section{C.1.3 Abilities}

The Non-knowledge abilities identified in the inventory are rated in terms of

- Basic familiarity

- Competency

- Mastery

\section{C.1.4 Summary of Rating Scale (Level by Type)}

- The following scale was used to rate the level of KSA needed for each type of KSA for each of the inventory items:

- Internal Knowledge of FACTS

1. No recollection or recognition of facts needed for successful performance of Task/KSAitem;

2. Recollection or recognition of facts is helpful, but not essential, for successful performance;

3. Essential to recognize that observed information accurately represents something known to be true;

4. Essential to recall basic facts from memory; and

5. Essential to recall or recognize basic facts from memory quickly.

- External Information about FACTS

1. No external information about facts is needed for successful performance of Task/KSAitem;

2. External information about facts is helpful, but not essential, for successful performance;

3. Essential to use external information to verify that observed information accurately represents something known to be true;

4. Essential to use external resources to access factual information; and

5. Essential to use external resources to quickly access factual information or verify observed information.

- Conceptual Knowledge

1. None - No conceptual knowledge is needed for successful performance of the Task/KSA item;

2. Understand - A fundamental level of comprehension, shown by the ability to explain, interpret, rephrase, or summarize the concept or make comparisons or classifications using the concept is needed for successful performance;

3. Analyze - A higher level of comprehension, enabling one to draw conclusions about a state of affairs using it, decompose something into its parts or functions using it, or integrate parts or functions into a whole using it, is necessary;

4. Evaluate - A still higher level of understanding of a concept that allows one to make good judgments about the truth, quality, or validity of something or a state of affairs is necessary; and 
5. Create - The highest level of understanding that allows one to create new concepts based on the concept that allows one to explain or predict something or a state of affairs better than has been done before is needed.

- Procedural Knowledge

1. No need to know for successful performance of the Task/KSA item

2. Only need to know the purpose of the thing being done;

3. Need to know how it is done, but not how to execute it;

4. Need to know how to execute it, but can be effortful, awkward, or slow;

5. Need fluency; and

6. Need to know how to teach fluency well.

- Psychomotor Skills (Motor, Visual, Auditory, and Tactile)

1. Not needed

2. Familiarity needed - need knowledge of how to carry out the skill for successful performance, but do not need to be able to execute the skill reliably

3. Controlled - need to be able to execute the skill reliability, but execution can require attention, can be slow, deliberate, and/or unpracticed

4. Automatic - need to be able to execute skill reliably with little attention to execution; execution needs to be quick, fluent, efficient, highly practiced.

\section{C.2 Results of the Level and Type of KSAs for each Task/KSA Inventory Item}

Table C.1. Ratings of Task/KSAs on Levels of Knowledges and Skills Needed

\begin{tabular}{|c|c|c|c|c|c|}
\hline $\mathbf{T}$ & & & Task/KSA Inventory Item & & \\
\hline p & $\begin{array}{l}\text { Level Required for Successful } \\
\text { Performance of Task }\end{array}$ & Knowledges & Ops & Mgt & Lic \\
\hline & CTS-Internal knowledge of facts & & & & \\
\hline 3 & $\begin{array}{l}\text { Essential to recognize that } \\
\text { observed information } \\
\text { accurately represents } \\
\text { something known to be true. }\end{array}$ & & & $\begin{array}{l}\text { Mgt-1; } \\
\text { Mgt-3; } \\
\text { Mgt-4 }\end{array}$ & \\
\hline 4 & $\begin{array}{l}\text { Essential to recall basic facts } \\
\text { from memory. }\end{array}$ & $\mathrm{K}-4 ; \mathrm{K}-5$ & $\begin{array}{l}\text { Ops-1; Ops-4b; Ops-7; Ops-10; } \\
\text { Ops-11; Ops-14; Ops-15 }\end{array}$ & $\begin{array}{l}\text { Mgt-2; } \\
\text { Mgt-5 }\end{array}$ & $\begin{array}{l}\text { Lic-1; } \\
\text { Lic-2 }\end{array}$ \\
\hline 5 & $\begin{array}{l}\text { Essential to recall or recognize } \\
\text { basic facts from memory } \\
\text { quickly. }\end{array}$ & & $\begin{array}{l}\text { Ops-2; Ops-3; Ops-4a; Ops-5; } \\
\text { Ops-6; Ops-8; Ops-9; Ops-12; } \\
\text { Ops-13; Ops-16 }\end{array}$ & & \\
\hline
\end{tabular}




\begin{tabular}{|c|c|c|c|c|c|}
\hline \multirow{2}{*}{$\begin{array}{l}\mathbf{T} \\
\mathbf{y} \\
\mathbf{p} \\
\mathbf{e}\end{array}$} & \multirow[b]{2}{*}{$\begin{array}{l}\text { Level Required for Successful } \\
\text { Performance of Task }\end{array}$} & \multicolumn{4}{|c|}{ Task/KSA Inventory Items } \\
\hline & & Knowledges & Ops & Mgt & Lic \\
\hline \multicolumn{6}{|c|}{ FACTS-External knowledge of facts } \\
\hline 3 & $\begin{array}{l}\text { Essential to use external } \\
\text { information to verify that } \\
\text { observed information } \\
\text { accurately represents } \\
\text { something known to be true. }\end{array}$ & & & $\begin{array}{l}\text { Mgt-1; } \\
\text { Mgt-3; } \\
\text { Mgt-4 }\end{array}$ & \\
\hline 4 & $\begin{array}{l}\text { Essential to use external resources } \\
\text { to access factual information. }\end{array}$ & $\mathrm{K}-4 ; \mathrm{K}-5$ & $\begin{array}{l}\text { Ops-1; Ops-4b; Ops-7; Ops-10; } \\
\text { Ops-11; Ops-14; Ops-15 }\end{array}$ & $\begin{array}{l}\text { Mgt-2; } \\
\text { Mgt-5 }\end{array}$ & $\begin{array}{l}\text { Lic-1; } \\
\text { Lic-2 }\end{array}$ \\
\hline 5 & $\begin{array}{l}\text { Essential to use external resources } \\
\text { to quickly access factual } \\
\text { information or verify observed } \\
\text { information. }\end{array}$ & & $\begin{array}{l}\text { Ops-2; Ops-3; Ops-4a; Ops-5; } \\
\text { Ops-6; Ops-8; Ops-9; Ops-12; } \\
\text { Ops-13; Ops-16 }\end{array}$ & & \\
\hline \multicolumn{6}{|c|}{ K-1; K-2; K-3; K-6; and K-7 (Plant Knowledge) [From the Task/KSA Inventory] } \\
\hline 1 & None (No knowledge needed) & & & & \\
\hline 2 & Understand & $\mathrm{K}-4 ; \mathrm{K}-5$ & All Ops- items & $\begin{array}{l}\text { Mgt-1; } \\
\text { Mgt-2; } \\
\text { Mgt-5 }\end{array}$ & $\begin{array}{l}\text { Lic-1; } \\
\text { Lic-2 }\end{array}$ \\
\hline \multicolumn{6}{|c|}{ PROCEDURAL Knowledge } \\
\hline 2 & $\begin{array}{l}\text { Only need to know the purpose of } \\
\text { the thing being done }\end{array}$ & & & Mgt-1 & \\
\hline 3 & $\begin{array}{l}\text { Need to know how it is done, but } \\
\text { not how to execute it. }\end{array}$ & K4; K-5 & $\begin{array}{l}\text { Ops-1; Ops-2; Ops-3; Ops-4a; } \\
\text { Ops-4b; Ops-5; Ops-6; Ops-8; } \\
\text { Ops-11; Ops-12; Ops-13; } \\
\text { Ops-14; Ops-15 }\end{array}$ & $\begin{array}{l}\text { Mgt-2; } \\
\text { Mgt-3; } \\
\text { Mgt-4 }\end{array}$ & $\begin{array}{l}\text { Lic-1; } \\
\text { Lic-2 }\end{array}$ \\
\hline 4 & $\begin{array}{l}\text { Need to know how to execute it, } \\
\text { but effortful, awkward, or slow. }\end{array}$ & & Ops-7; Ops-9; Ops-10; Ops-16 & Mgt-5 & \\
\hline \multicolumn{6}{|c|}{ PSYCHOMOTOR Skills Motor } \\
\hline 1 & Not needed & $\mathrm{K}-4 ; \mathrm{K}-5$ & All & All & $\begin{array}{l}\text { Lic-1; } \\
\text { Lic-2 }\end{array}$ \\
\hline 2 & Familiarity Needed & & & & \\
\hline \multicolumn{6}{|c|}{ PSYCHOMOTOR Skills Visual } \\
\hline 1 & Not needed & $\mathrm{K}-4 ; \mathrm{K}-5$ & & Mgt-3 & \\
\hline 2 & Familiarity Needed & & All & $\begin{array}{l}\text { Mgt-1; } \\
\text { Mgt-2; } \\
\text { Mgt-4; } \\
\text { Mgt-5 }\end{array}$ & $\begin{array}{l}\text { Lic-1; } \\
\text { Lic-2 }\end{array}$ \\
\hline \multicolumn{6}{|c|}{ PSYCHOMOTOR Skills Auditory } \\
\hline 1 & Not needed & $\mathrm{K}-4 ; \mathrm{K}-5$ & & $\begin{array}{l}\text { Mgt-4; } \\
\text { Mgt-5 }\end{array}$ & \\
\hline 2 & Familiarity Needed & & All & $\begin{array}{l}\text { Mgt-1; } \\
\text { Mgt-2; } \\
\text { Mgt-3; }\end{array}$ & $\begin{array}{l}\text { Lic-1; } \\
\text { Lic-2 }\end{array}$ \\
\hline
\end{tabular}




\begin{tabular}{|c|c|c|c|c|c|}
\hline \multirow{2}{*}{$\begin{array}{l}\mathbf{T} \\
\mathbf{y} \\
\mathbf{p} \\
\mathbf{e}\end{array}$} & \multirow[b]{2}{*}{$\begin{array}{l}\text { Level Required for Successful } \\
\text { Performance of Task }\end{array}$} & \multicolumn{4}{|c|}{ Task/KSA Inventory Items } \\
\hline & & Knowledges & Ops & Mgt & Lic \\
\hline \multicolumn{6}{|c|}{ PSYCHOMOTOR Skills Tactile } \\
\hline 1 & Not needed & $\mathrm{K}-4 ; \mathrm{K}-5$ & All & All & $\begin{array}{l}\text { Lic-1; } \\
\text { Lic-2 }\end{array}$ \\
\hline 2 & Familiarity Needed & & & & \\
\hline
\end{tabular}

C. 7 


\section{Appendix D}

\section{Reactor and Simulator CR Systems and Elements and Their Importance to Task/KSA Inventory Items}




\section{Appendix D}

\section{Reactor and Simulator CR Systems and Elements and Their Importance to Task/KSA Inventory Items}

\section{D.1 Results of the Importance of Systems to Task/KSA Inventory Items Analysis}

Table D.1. Rating of the Importance of Each HSI System to Each Task/KSA Item

\begin{tabular}{|c|c|c|c|c|c|c|c|c|}
\hline $\begin{aligned} 1 & =\underset{\text { Not }}{\text { important }} \\
2 & =\underset{\text { Somewhat }}{\text { important }} \\
3 & =\text { Important }\end{aligned}$ & 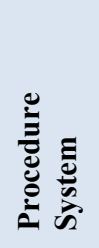 & 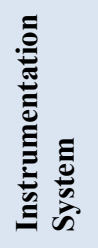 & 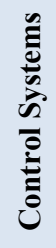 & 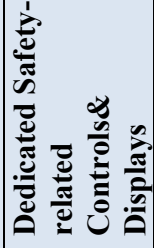 & 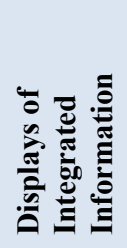 & 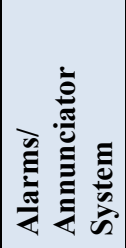 & 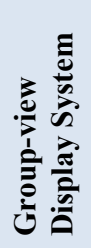 & 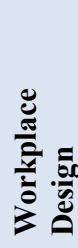 \\
\hline Ops-1 RI & 1 & 3 & 1 & 3 & 3 & 2 & 3 & 2 \\
\hline Ops-1 OLE & 1 & 3 & 1 & 3 & 3 & 2 & 3 & 2 \\
\hline Ops-2 RI & 3 & 3 & 3 & 2 & 2 & 2 & 2 & 2 \\
\hline Ops-2 OLE & 3 & 3 & 3 & 2 & 2 & 2 & 2 & 2 \\
\hline Ops-3 RI & 3 & 3 & 3 & 2 & 2 & 2 & 2 & 2 \\
\hline Ops-3 OLE & 3 & 3 & 3 & 2 & 2 & 2 & 2 & 2 \\
\hline Ops-4a RI & 2 & 3 & 2 & 3 & 2 & 2 & 1 & 1 \\
\hline $\begin{array}{l}\text { Ops-4b RI } \\
\text { Ops-4b OLE }\end{array}$ & $\begin{array}{l}2 \\
2\end{array}$ & $\begin{array}{l}3 \\
3 \\
\end{array}$ & $\begin{array}{l}3 \\
3 \\
\end{array}$ & $\begin{array}{l}3 \\
3 \\
\end{array}$ & $\begin{array}{l}2 \\
2 \\
\end{array}$ & $\begin{array}{l}2 \\
3 \\
\end{array}$ & $\begin{array}{l}1 \\
1\end{array}$ & $\begin{array}{l}2 \\
2 \\
\end{array}$ \\
\hline $\begin{array}{l}\text { Ops-5 RI } \\
\text { Ops-5 OLE }\end{array}$ & $\begin{array}{l}2 \\
2\end{array}$ & $\begin{array}{l}3 \\
3 \\
\end{array}$ & $\begin{array}{l}2 \\
2\end{array}$ & $\begin{array}{l}3 \\
3 \\
\end{array}$ & $\begin{array}{l}3 \\
3 \\
\end{array}$ & $\begin{array}{l}2 \\
2\end{array}$ & $\begin{array}{l}2 \\
2\end{array}$ & $\begin{array}{l}1 \\
1\end{array}$ \\
\hline Ops-6 RI & 3 & 3 & 3 & 3 & 3 & 3 & 2 & 1 \\
\hline Ops-6 OLE & 3 & 3 & 3 & 3 & 3 & 3 & 2 & 1 \\
\hline Ops-7 RI & 3 & 3 & 3 & 1 & 1 & 2 & 2 & 2 \\
\hline Ops-7 OLE & 3 & 3 & 3 & 1 & 1 & 2 & 2 & 2 \\
\hline Ops-8 RI & 3 & 3 & 3 & 3 & 3 & 3 & 3 & 2 \\
\hline Ops-8 OLE & 3 & 3 & 3 & 3 & 3 & 3 & 3 & 2 \\
\hline Ops-9 RI & 3 & 3 & 3 & 3 & 3 & 3 & 3 & 3 \\
\hline Ops-9 OLE & 3 & 3 & 3 & 3 & 3 & 3 & 3 & 3 \\
\hline Ops-10 RI & 3 & 3 & 1 & 3 & 3 & 3 & 2 & 1 \\
\hline Ops-10 OLE & 3 & 3 & 1 & 3 & 3 & 3 & 2 & 1 \\
\hline Ops-11 RI & 3 & 1 & 3 & 1 & 1 & 1 & 2 & 2 \\
\hline Ops-11 OLE & 3 & 1 & 3 & 1 & 1 & 1 & 2 & 2 \\
\hline Ops-12 RI & 3 & 3 & 3 & 2 & 2 & 3 & 2 & 1 \\
\hline Ops-12 OLE & 3 & 3 & 3 & 2 & 2 & 3 & 2 & 1 \\
\hline Ops-13 RI & 3 & 3 & 3 & 3 & 3 & 2 & 2 & 1 \\
\hline Ops-13 OLE & 3 & 3 & 3 & 3 & 3 & 2 & 2 & 1 \\
\hline
\end{tabular}




\begin{tabular}{|c|c|c|c|c|c|c|c|c|}
\hline $\begin{aligned} 1 & =\underset{\text { Not }}{\text { important }} \\
2 & =\begin{array}{r}\text { Somewhat } \\
\text { important }\end{array} \\
3 & =\text { Important }\end{aligned}$ & 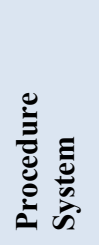 & 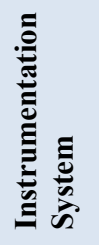 & 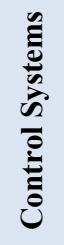 & 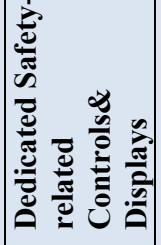 & 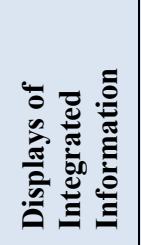 & 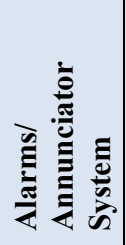 & 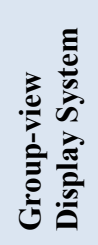 & 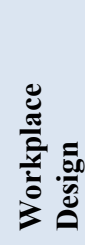 \\
\hline Ops-14b RI & 1 & 3 & 3 & 3 & 1 & 1 & 1 & 2 \\
\hline Ops-14b OLE & 1 & 3 & 3 & 3 & 1 & 1 & 1 & 2 \\
\hline Ops-15 RI & 3 & 3 & 3 & 3 & 2 & 3 & 3 & 2 \\
\hline Ops-15 OLE & 3 & 3 & 3 & 3 & 2 & 3 & 3 & 2 \\
\hline Ops-16 RI & 3 & 3 & 3 & 3 & 3 & 3 & 2 & 2 \\
\hline Ops-16 OLE & 3 & 3 & 3 & 3 & 3 & 3 & 2 & 2 \\
\hline Mgmt-1 RI & 3 & 1 & 1 & 1 & 1 & 1 & 3 & 3 \\
\hline Mgmt-1 OLE & 3 & 1 & 1 & 1 & 1 & 1 & 3 & 3 \\
\hline Mgmt-2 RI & 3 & 3 & 1 & 1 & 1 & 2 & 3 & 3 \\
\hline Mgmt-2 OLE & 3 & 3 & 1 & 1 & 1 & 2 & 3 & 3 \\
\hline Mgmt-3 RI & 1 & 1 & 1 & 1 & 1 & 1 & 2 & 3 \\
\hline Mgmt-3 OLE & 1 & 1 & 1 & 1 & 1 & 1 & 2 & 3 \\
\hline Mgmt-4 RI & 3 & 2 & 2 & 2 & 1 & 2 & 1 & 3 \\
\hline Mgmt-4 OLE & 3 & 2 & 2 & 2 & 1 & 2 & 1 & 3 \\
\hline Mgmt-5 RI & 3 & 1 & 2 & 2 & 1 & 1 & 1 & 1 \\
\hline Mgmt-5 OLE & 3 & 1 & 2 & 2 & 1 & 1 & 1 & 1 \\
\hline Lic-1 RI & 3 & 1 & 2 & 2 & 1 & 1 & 1 & 1 \\
\hline Lic-1 OLE & 3 & 1 & 2 & 2 & 1 & 1 & 1 & 1 \\
\hline Lic-2 RI & 3 & 3 & 3 & 3 & 3 & 3 & 3 & 1 \\
\hline Lic-2 OLE & 3 & 3 & 3 & 3 & 3 & 3 & 3 & 1 \\
\hline K-4 RI & 3 & 1 & 1 & 1 & 1 & 1 & 1 & 2 \\
\hline K-4 OLE & 3 & 1 & 1 & 1 & 1 & 1 & 1 & 2 \\
\hline K-5 RI & 3 & 1 & 1 & 1 & 1 & 1 & 1 & 2 \\
\hline K-5 OLE & 3 & 1 & 1 & 1 & 1 & 1 & 1 & 2 \\
\hline
\end{tabular}


Table D.2. Preliminary Taxonomy of CR HSI and Simulator Systems and Elements

\begin{tabular}{|c|c|c|c|c|c|c|c|c|}
\hline \multicolumn{7}{|c|}{ Elements of Control Room Simulators and the Basis for Fidelity Comparisons } & \multicolumn{2}{|c|}{ Assumed Technology } \\
\hline $\begin{array}{c}\text { System } \\
\text { Control } \\
\text { Panels } \\
\end{array}$ & Display & $\begin{array}{c}\text { Input } \\
\text { Devices }\end{array}$ & Alarms & $\begin{array}{c}\text { Decision } \\
\text { Aides }\end{array}$ & $\begin{array}{c}\text { Commun- } \\
\text { ication } \\
\text { Equipment }\end{array}$ & $\begin{array}{c}\text { Workplace } \\
\text { Design }\end{array}$ & $\begin{array}{c}\text { Simulator } \\
\text { Model }\end{array}$ & $\begin{array}{c}\text { User - System } \\
\text { Interaction }\end{array}$ \\
\hline Labeling & Video & Keyboards & General Display & SPDS & Telephones & Stand-up Console & Data Quality & Command Language \\
\hline Gauges & $\begin{array}{l}\text { Flat Panel - Large } \\
\text { Screen }\end{array}$ & $\begin{array}{l}\text { Function } \\
\text { Keys }\end{array}$ & Alarm Messages & $\begin{array}{l}\text { Plant } \\
\text { Computer }\end{array}$ & $\begin{array}{l}\text { Sound Powered } \\
\text { Phones }\end{array}$ & Sit-Down Console & Response Rate & Menu Selection \\
\hline CRTs & Printer/Recorders & Track balls & $\begin{array}{l}\text { Organization of } \\
\text { Alarms }\end{array}$ & $\begin{array}{l}\text { Reference } \\
\text { Material \& } \\
\text { Procedures }\end{array}$ & Portable radios & $\begin{array}{l}\text { Sit-Stand } \\
\text { Workstation }\end{array}$ & Freeze Frame & Function Keys \\
\hline $\begin{array}{l}\text { Valve Position } \\
\text { Indications }\end{array}$ & Audio Display & Joy Sticks & Silence Control & & Paging Systems & Vertical Panels & Back Track & Forms \\
\hline $\begin{array}{l}\text { Manual Valve } \\
\text { Control }\end{array}$ & $\begin{array}{l}\text { Analog/Digital } \\
\text { Meters }\end{array}$ & Mice & $\begin{array}{l}\text { Acknowledge } \\
\text { Control }\end{array}$ & & $\begin{array}{l}\text { Emergency } \\
\text { Communi-cation } \\
\text { Systems }\end{array}$ & Desks & Fast Forward & Cursers \\
\hline Environment & Light Indicators & $\begin{array}{l}\text { Touch } \\
\text { Screens }\end{array}$ & Reset Control & & & Chairs & Malfunctions & Prompts \\
\hline $\begin{array}{l}\text { Communication } \\
\text { Systems }\end{array}$ & Numeric Readouts & $\begin{array}{l}\text { Graphic } \\
\text { Tables }\end{array}$ & Test Function & & & $\begin{array}{l}\text { Furniture \& } \\
\text { Equipment Layout }\end{array}$ & System Interface & Feedback \\
\hline Pump Control & Mimics & $\begin{array}{l}\text { Speech Input } \\
\text { Devices }\end{array}$ & Failure Indication & & & Environment & & Advisory Messages \\
\hline Breaker Control & $\begin{array}{l}\text { Graphic Instrument } \\
\text { Displays }\end{array}$ & Push Buttons & Audible Indication & & & Panel Layout & & Error Messages \\
\hline Alarms & $\begin{array}{l}\text { Non-Safety \& Safety } \\
\text { Grade Indication }\end{array}$ & $\begin{array}{l}\text { Rotary } \\
\text { Controls } \\
\end{array}$ & & & & Panel Design & & $\begin{array}{l}\text { System Response } \\
\text { Time }\end{array}$ \\
\hline Panels & Analog Indication & $\begin{array}{l}\text { Analog } \\
\text { Controls }\end{array}$ & & & & Panel Labeling & & $\begin{array}{l}\text { Display Selection \& } \\
\text { Navigation }\end{array}$ \\
\hline Consoles & Digital Indication & $\begin{array}{l}\text { Digital } \\
\text { Controls } \\
\end{array}$ & & & & $\begin{array}{l}\text { Emergency } \\
\text { Equipment }\end{array}$ & & Display Controls \\
\hline & Paper recorders & Switches & & & & $\begin{array}{l}\text { Reference Material } \\
\text { \& Document } \\
\text { Organization \& } \\
\text { Storage }\end{array}$ & & $\begin{array}{l}\text { Saving Files \& } \\
\text { Information }\end{array}$ \\
\hline & Digital Recorders & & & & & & & Protection of Data \\
\hline & & & & & & & & System Security \\
\hline & & & & & & & & Malfunctions \\
\hline
\end{tabular}


Table D.3. Lesson Plan Cross-Walk with Task/KSA Inventory Items

\begin{tabular}{|c|c|c|c|}
\hline \multicolumn{3}{|c|}{$\begin{array}{l}\text { Lesson \# 624P-1.2 Title: System Review - Rod Control \& Excore } \\
\text { Instrumentation }\end{array}$} & \multirow{2}{*}{$\begin{array}{l}\text { Applicable Advanced Reactor System - } \\
\text { Knowledges/Skills/Abilities }\end{array}$} \\
\hline Objectives & Prese & ation: & \\
\hline $\begin{array}{l}\text { 1.1 Provide the student with } \\
\text { the following detailed } \\
\text { information concerning the } \\
\text { Rod Control System and the } \\
\text { Excore Nuclear } \\
\text { Instrumentation System: } \\
\text { 1.1.1 Controls that be } \\
\text { manipulated by the student } \\
\text { during the course of a } \\
\text { reactor startup, shutdown, } \\
\text { and /or power operation. } \\
\text { 1.1.2 Control switches that } \\
\text { must be operated to block } \\
\text { reactor trip signals or to } \\
\text { inhibit/bypass faulted } \\
\text { instruments from various } \\
\text { automatic control stations. } \\
\text { 1.1.3 Indications available to } \\
\text { the student from this } \\
\text { location used to evaluate the } \\
\text { status of the core. }\end{array}$ & $\begin{array}{l}\text { Ops-7 } \\
\text { Lic-1 } \\
\text { Ops-5 } \\
\text { Ops-6 }\end{array}$ & $\begin{array}{l}\text { A. Controls (Show use and location of } \\
\text { the following) } \\
\text { 1. Rod bank auto/manual selector } \\
\text { 2. IN-HOLD-OUT switch } \\
\text { 3. Source range block/reset } \\
\text { 4. Intermediate range block } \\
\text { 5. Power range block } \\
\text { 6. Startup reset } \\
\text { 7. Rod control alarm reset } \\
\text { 8. Tavg and } \Delta \text { T defeat switches } \\
\text { 9. Both manual trip switches } \\
\text { B. Instrumentation (Show use and } \\
\text { location of the following) } \\
\text { 1. Source range count and SUR } \\
\text { 2. Intermediate range current and SUR } \\
\text { 3. Power range level and } \Delta \text { flux } \\
\text { 4. Step counters } \\
\text { 5. RCS temperature } \\
\text { a. OP } \mathrm{T} \\
\text { b. OT } \triangle \mathrm{T} \\
\text { c. } \Delta \mathrm{T} \\
\text { d. Tavg } \\
\text { 6. NR-45 recorder } \\
\text { 7. Tref/Tavg auctioneered } \\
\text { C. Annunciation to be aware of during } \\
\text { startup } \\
\text { 1. SR HI FLUX AT S/D BLOC } \\
\text { 2. ROD BOTTOM ANNUNCIATION } \\
\text { 3. SR HI VOLT FAIL } \\
\text { 4. ROD BANK LIMIT LO } \\
\text { 5. ROD BANK LIMIT LO-LO } \\
\text { D. Associated Technical Specifications } \\
\text { 1. Control Rods } \\
\text { a. Control rod operability } \\
\text { b. Rod position indication } \\
\text { c. Rod drop times } \\
\text { d. Rod insertion limits } \\
\text { 2. Excore Nuclear Instruments } \\
\text { a. Axial Flux Difference } \\
\text { b. Quadrant Power Tilt Ratio } \\
\text { c. Reactor Protection System }\end{array}$ & 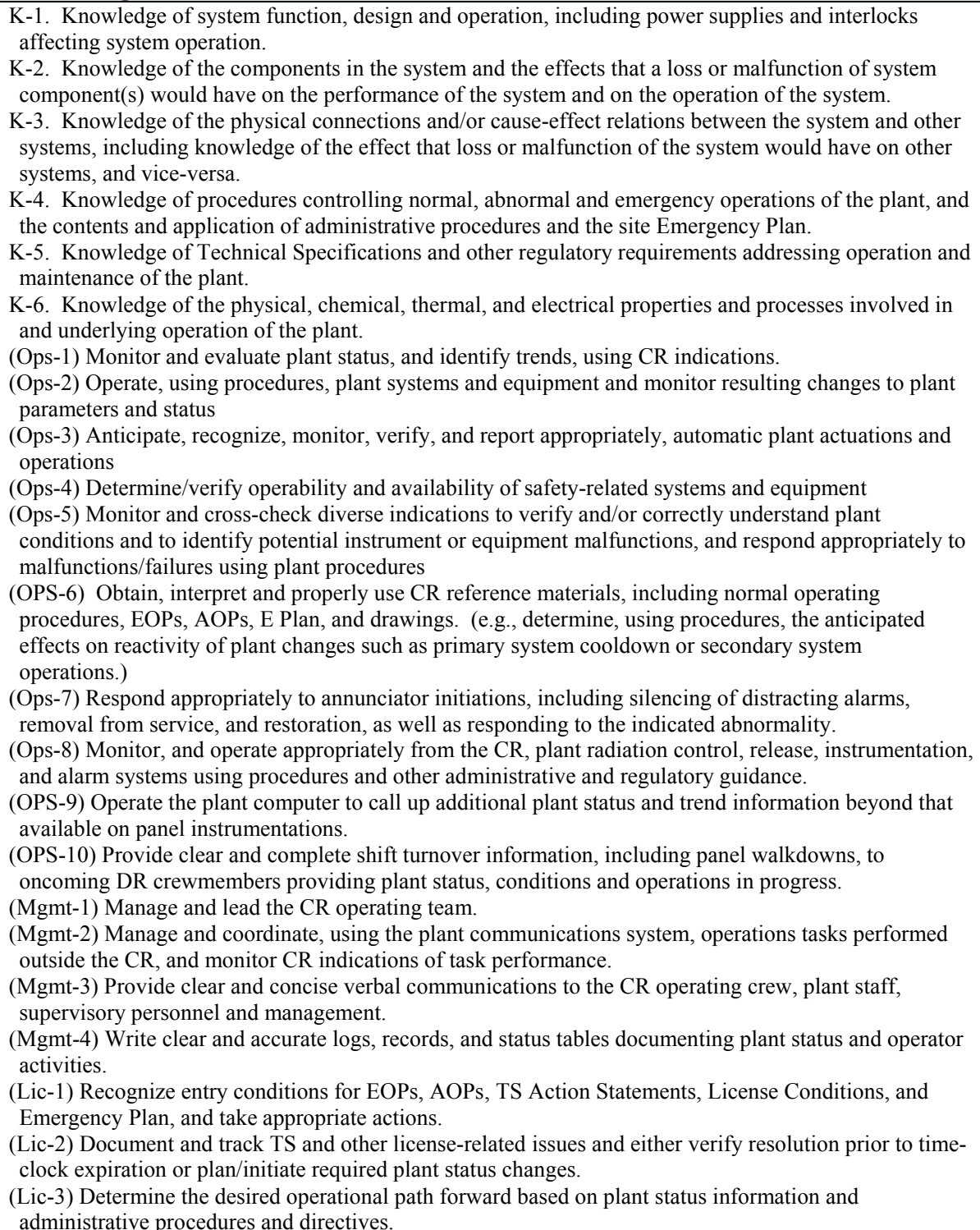 \\
\hline
\end{tabular}


Table D.3. (contd)

\begin{tabular}{|c|c|c|c|}
\hline \multicolumn{3}{|c|}{$\begin{array}{l}\text { Lesson \# 624P-1.3 Title: System Review - Bistable Status Panel } \\
\text { Time: } 60 \text { Minutes }\end{array}$} & Applicable Advanced Reactor System - \\
\hline Objectives & Prese & tation: & Applicable Knowledges/Skills/Abilities \\
\hline $\begin{array}{l}\text { 1.1 Provide the student with } \\
\text { information concerning the } \\
\text { following: } \\
\text { 1.1.1 Use of procedures } \\
\text { 1.1.2 Layout of the General } \\
\text { Operating Instructions (GOIs) } \\
\text { 1.1.3 Precautions and limitations to } \\
\text { follow during startup } \\
\text { 1.1.4 License limits } \\
\text { 1.1.5 Information available from } \\
\text { the protection system permissive } \\
\text { status panel and the bypass } \\
\text { status panel. } \\
\text { Simulator IC, MALF, LOA, } \\
\text { or TS Item } \\
\text { INIT } 8 \\
\text { (NOTE: IC } 7 \text { is unstable) } \\
\text { GOI 2-1 is complete until MFP is } \\
\text { desired. } \\
\text { This procedure has } 25 \text { precautions. } \\
\text { These precautions should be discussed } \\
\text { in some detail before a Rx S/U. } \\
\text { \{TR-Table 5.1.2-1\} } \\
\text { \{TS 3.1.5 } \\
\text { \{TS 3.1.6\} } \\
\text { \{TS 3.1.7\} } \\
\text { \{TS 3.1.8\} } \\
\text { \{TS 2.1.1\} } \\
\text { \{TS 3.4.2\} } \\
\text { \{TS 3.4.1\} } \\
\text { Introduce the use of control room logic } \\
\text { drawings [M1T-13 (1 thru 16)] }\end{array}$ & $\begin{array}{l}\text { K-1 } \\
\text { K-2 } \\
\text { K-3 } \\
\text { Ops-7 } \\
\text { Ops-6 }\end{array}$ & 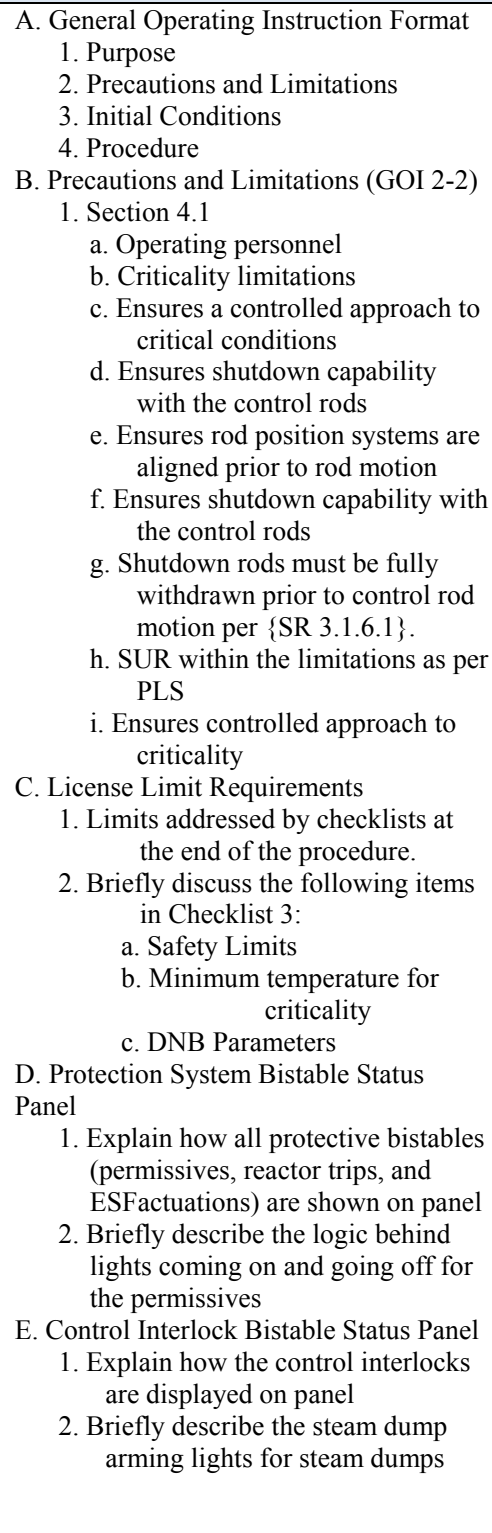 & 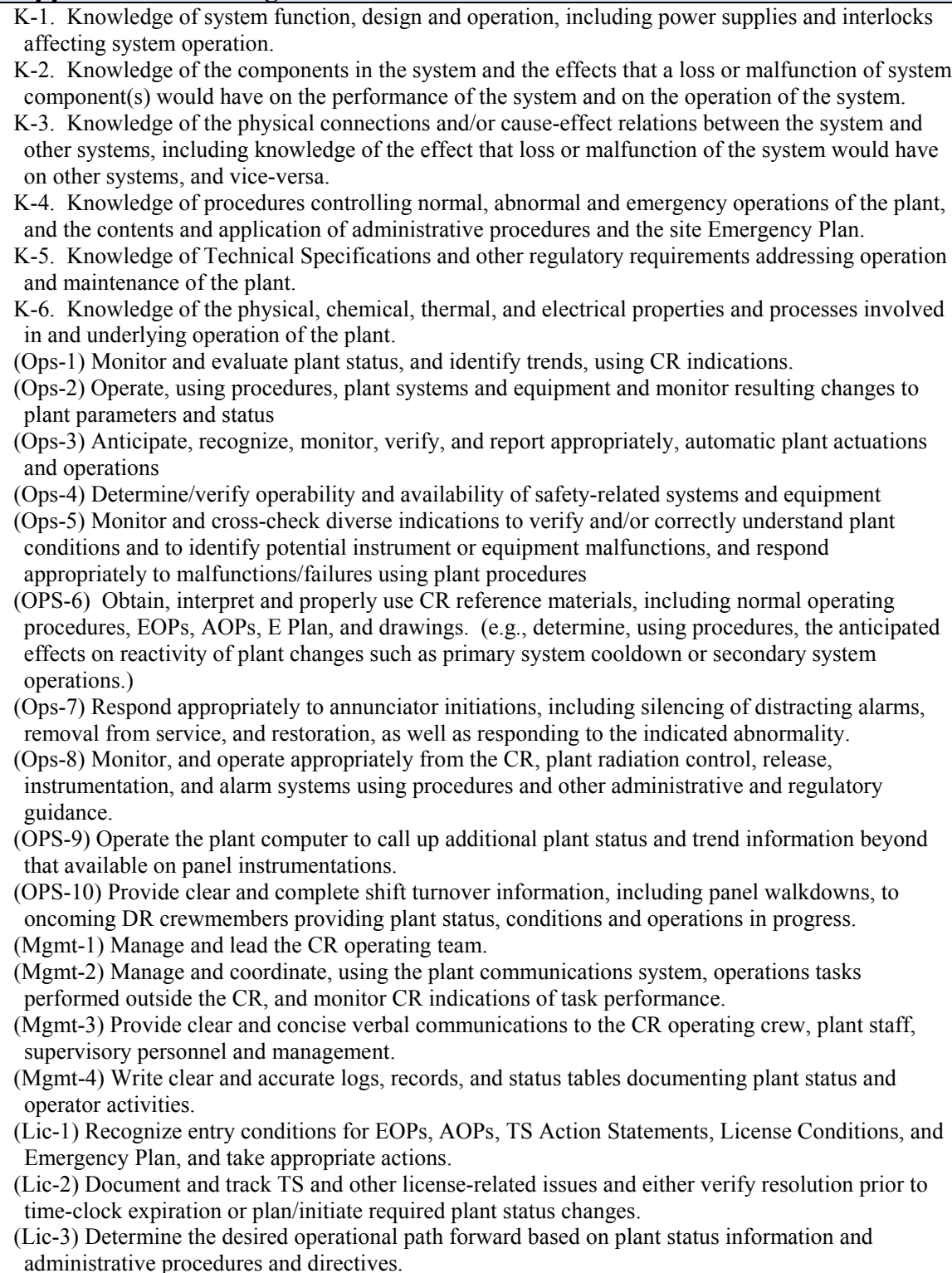 \\
\hline
\end{tabular}


Table D.3. (contd)

\begin{tabular}{|c|c|c|c|}
\hline \multicolumn{3}{|c|}{$\begin{array}{l}\text { Lesson \# 624P-1.4 Title: Reactor Startups } \\
\text { Time: } 4 \text { Hours }\end{array}$} & \multirow{2}{*}{$\begin{array}{l}\text { Applicable Advanced Reactor System - } \\
\text { Knowledges/Skills/Abilities }\end{array}$} \\
\hline Objectives & Presen & tation: & \\
\hline $\begin{array}{l}\text { 1.1 Provide simulator orientation to the } \\
\text { student } \\
\text { 1.2 Provide the student with the } \\
\text { following: } \\
\text { 1.2.1 An understanding of how the core } \\
\text { is made critical. } \\
\text { 1.2.2 How to recognize criticality. } \\
\text { 1.2.3 How to control the reactivity of } \\
\text { the core. } \\
\text { 1.2.4 Effects of subcritical } \\
\text { multiplication. } \\
\text { 1.2.5 Technical specifications that may } \\
\text { be entered during rod withdrawal. } \\
\text { Simulator IC, MALF, LOA, or TS } \\
\text { Item } \\
\text { INIT } 8 \\
\text { OI 8-1 } \\
\text { PLP RCS } 1 \\
\text { PZR } 2 \\
\text { CVC } 3 \\
\text { \{TS 3.4.2\} } \\
\text { CTS 3.1.7\} } \\
\text { CROC\&TRM } \\
\text { Figure 1.20 }\end{array}$ & $\begin{array}{c}\text { Ops-2 } \\
\text { Mgmt- } \\
4 \\
\text { Ops-10 } \\
\text { Ops-2 } \\
\text { Ops-1 } \\
\text { Ops-5 } \\
\text { Ops-7 } \\
\text { Mgmt- } \\
1 \\
\text { Mgmt- } \\
2 \\
\text { Mgmt- } \\
3 \\
\text { K-1 }\end{array}$ & $\begin{array}{l}\text { NOTE: It takes approximately } 30 \text { minutes } \\
\text { to perform each startup and escalate power } \\
\text { to } 5 \mathrm{E}-10 \text { amps.A. Each student should take } \\
\text { the reactor critical and escalate power to at } \\
\text { least } 5 \mathrm{E}-10 \text { amps. } \\
\text { 1. If the class is small ( } 3 \text { or } 4 \text { ) power may } \\
\text { be raised to POAH. In any case, the last } 2 \text { or } \\
3 \text { startups should be raising power to at least } \\
2 \% \text { and starting a main feedwater pump. } \\
\text { B. Have students not directly involved with } \\
\text { the startup complete the checklists as } \\
\text { referenced on the cover sheet. } \\
\text { C. Perform reactor startup IAW GOI } 2-2 \\
\text { D. Provide students with ECC } \\
\text { 1. } 120 \text { steps on bank D for first startup } \\
\text { 2. For subsequent startups, vary the } \\
\text { boric acid concentration in the RCS to } \\
\text { vary the critical rod position. A change } \\
\text { of } \pm 1 \text { ppm will change critical rod } \\
\text { position by approximately } 2 \text { steps. } \\
\text { E. Explain how to recognize criticality } \\
\text { 1. Describe how the count rate at } \\
\text { criticality can be changed by } \\
\text { allowing subcritical multiplication to } \\
\text { increase counts. } \\
\text { a. Recall that theoretically the core can be } \\
\text { taken to } 100 \% \text { power without achieving } \\
\text { criticality. However, in practice, this cannot } \\
\text { be accomplished due to rod worth } \\
\text { F. Ensure the students are aware of the } \\
\text { following Technical Specification items: } \\
\text { 1. Minimum temperature for criticality. } \\
\text { 2. Rod insertion limits } \\
\text { G. Startup Demonstration } \\
\text { 1. Initial Conditions } \\
\text { a. Have students take the reactor critical } \\
\text { and level power at } 5 \mathrm{E}-10 \text { amps. } \\
\text { b. Pull rods to achieve } 1 / 2 \text { dpm SUR } \\
\text { c. Ask students to determine final power } \\
\text { 1) Monitor power on indications } \\
\text { 2) Note point of doppler and MTC feedback } \\
\text { 3) Calculate reactivity added by rod motion } \\
\text { for final SUR } \\
\text { 4) Use power defect curve to determine } \\
\text { final power }\end{array}$ & 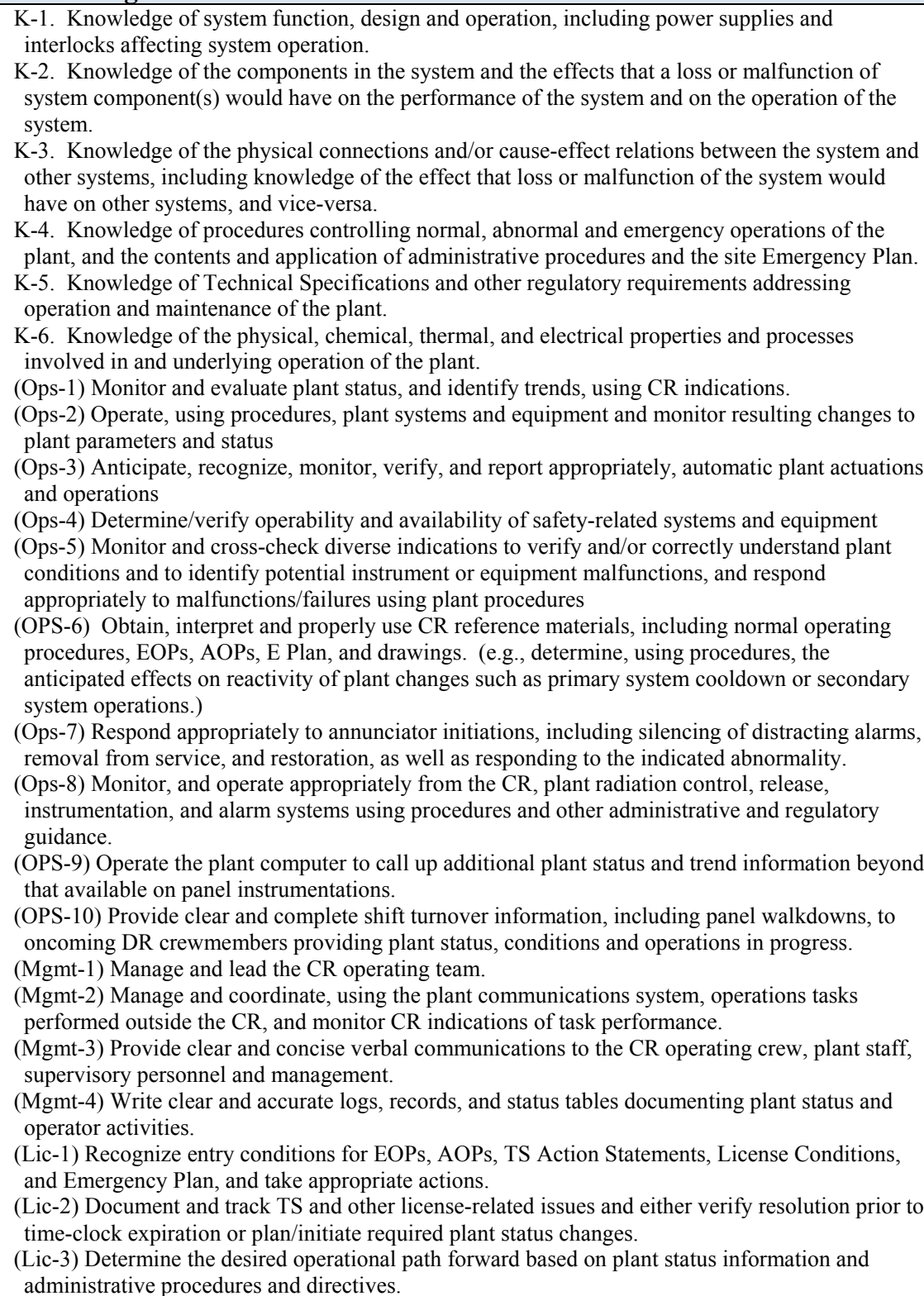 \\
\hline
\end{tabular}


Table D.3. (contd)

\begin{tabular}{|c|c|c|c|}
\hline \multicolumn{3}{|c|}{$\begin{array}{l}\text { Lesson \# 624P-1.5 Title: System Review - Condensate, Feedwater, } \\
\text { Auxiliary Feedwater, \& Steam Generator Water Level Control } \\
\text { Time: } 60 \text { Minutes }\end{array}$} & \multirow{2}{*}{$\begin{array}{l}\text { Applicable Advanced Reactor System - } \\
\text { Applicable Knowledges/Skills/Abilities }\end{array}$} \\
\hline Objectives & Presen & tion: & \\
\hline 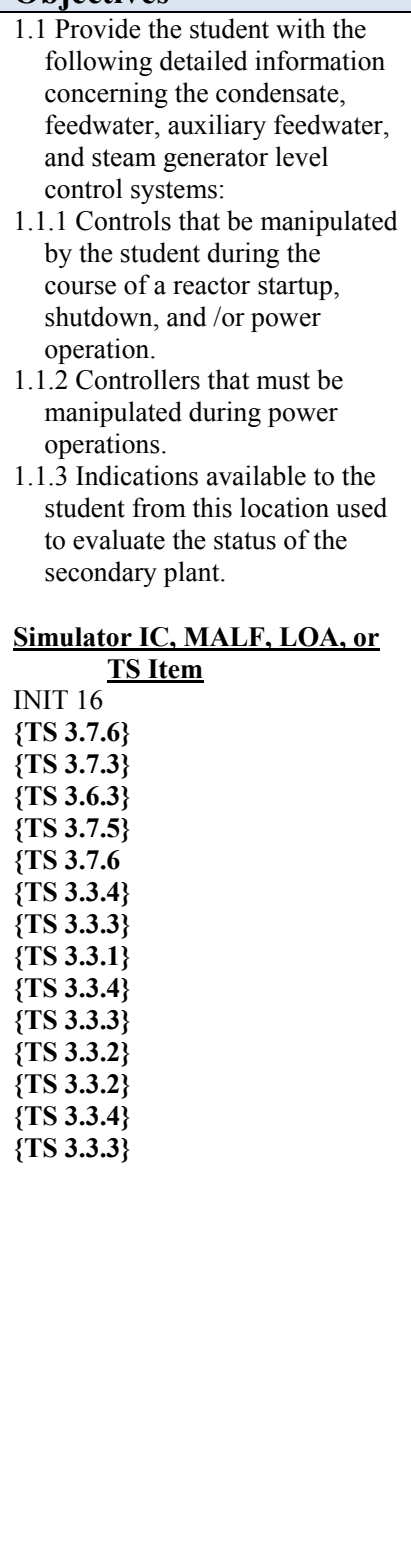 & $\begin{array}{c}\text { K-1 } \\
\text { Ops-5 } \\
\text { K-5 } \\
\text { Lic-1 } \\
\text { Lic-2 } \\
\text { Ops-8 }\end{array}$ & $\begin{array}{l}\text { A. System Description } \\
\text { 1. Use diagram to show flowpath of the } \\
\text { water from the sources to the } \mathrm{S} / \mathrm{G} \\
\text { B. Controls (Show the location and } \\
\text { operation of the following:) } \\
\text { 1. Aux feed pump S/G level control } \\
\text { valves } \\
\text { 2. MFW bypass control valves } \\
\text { 3. MFW control valves } \\
\text { 4. Reactor trip switches (both) } \\
\text { 5. Feed pump controls } \\
\text { a. Point out general location, a more } \\
\text { detailed description will be provided } \\
\text { during feed pump startup. } \\
\text { 6. Feed isolation valves and B/P valves } \\
\text { indications, and FWIS Reset } \\
\text { 7. Block switches for steam line flow SI } \\
\text { C. Instrumentation } \\
\text { 1. Location and use of the following: } \\
\text { a. S/G level indicators } \\
\text { b. S/G pressure indicators } \\
\text { c. Condensate storage tank level } \\
\text { d. Aux feed flow to S/G } \\
\text { e. AFW pumps indications (pressure, } \\
\text { flow, amps, steam chest pressure, } \\
\text { etc.) } \\
\text { 2. Show the location of the S/G feed, } \\
\text { steam, and level recorders } \\
\text { D. Technical Specifications } \\
\text { 1. Feedwater System } \\
\text { a. Feedwater isolation valves } \\
\text { 2. Auxiliary Feedwater } \\
\text { a. AFW pumps } \\
\text { b. Condensate storage tank } \\
\text { c. AFW instrumentation } \\
\text { 1) Remote shutdown panels } \\
\text { 2) Accident monitoring } \\
\text { 3. Steam Generator Level Control } \\
\text { a. Steam generator level } \\
\text { 1) RPS } \\
\text { 2) Remote Shutdown } \\
\text { 3) Accident monitoring } \\
\text { 4) ESF related signals } \\
\text { b. Steam Generator pressure } \\
\text { 1) ESFAS } \\
\text { 2) Remote shutdown } \\
\text { 3) Accident monitoring }\end{array}$ & 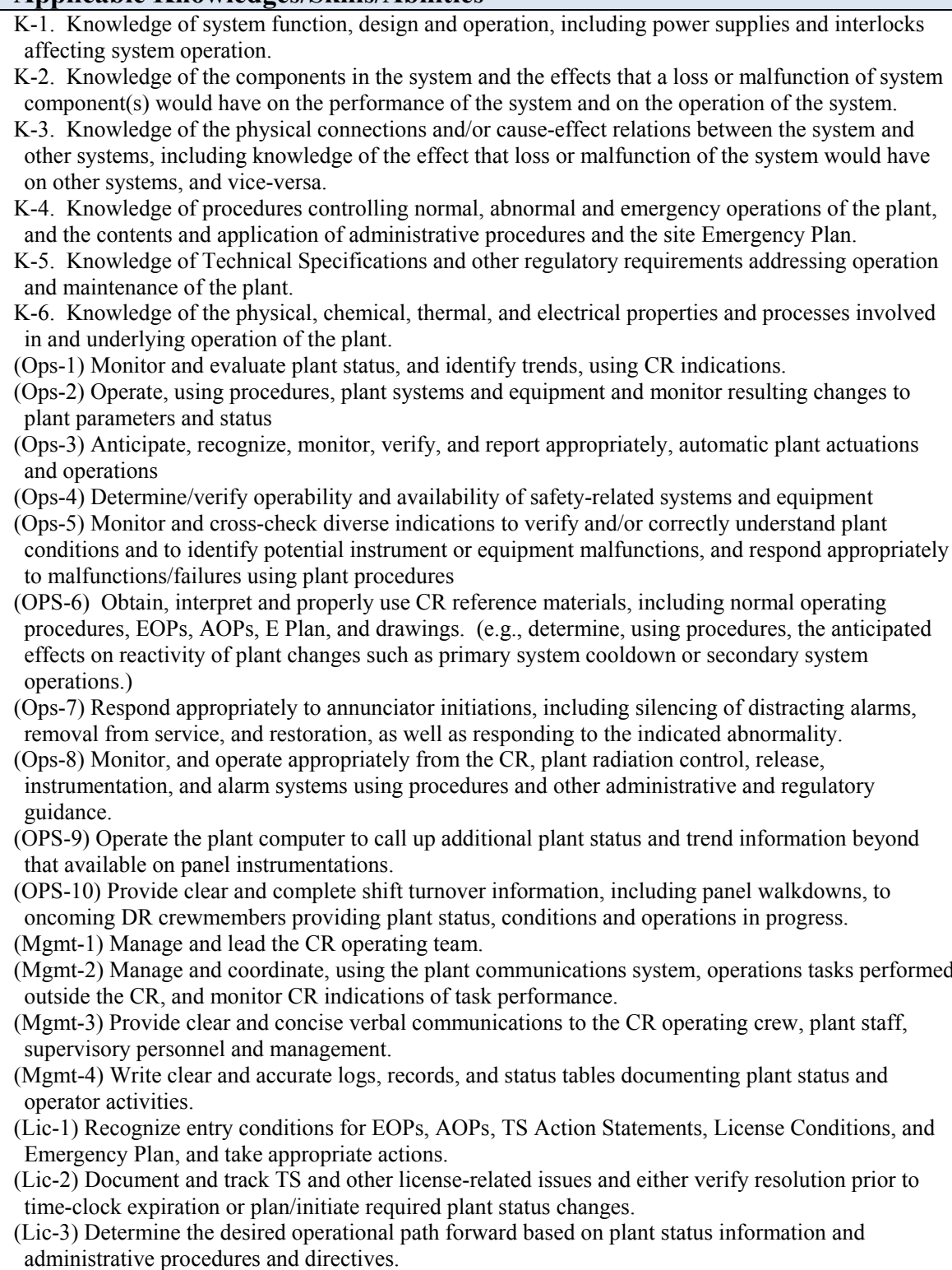 \\
\hline
\end{tabular}


Table D.3. (contd)

Lesson \# 624P-1.6 Title: Conduct Of Control Room Operations

Time: 30 Minutes

\section{Objectives}

Presentation:

1.1 Provide simulator operations orientation to the student 1.2 Provide the student with the following:

1.2.1 An understanding of control room command structure.

1.2.2 An understanding of control room personnel responsibilities.

1.2.3 Actions to be taken fo instrument failures.

1.2.4 Actions to be taken for alarm response.

1.2.5 An understanding of "3-way" communications

Attachment 1, Typical Control Room Organizational Chart

Note: Organization shown is typical at many plants. Point out to students that the organization and position titles will differ from site to site.

\section{Simulator IC, MALF, LOA, or TS} Item

K-4

Mgmt-1

Mgmt-2

Mgmt-3

Mgmt-4

\section{A. Control Room Organization:}

Review typical control room organization using Attachment 1.

K-5 B. Control room personnel responsibilities:

1. Shift Supervisor/Manager

a. The Shift Supervisor (SS) is the senior licensed individual on the shift (SRO) and is responsible for on-shift management and oversight of all plant group activities and has the authority to take action necessary to ensure compliance with Technical Specifications, operating license requirements, and approved plant procedures to protect the health and safety of employees and the public, to ensure adequate security, and to protect the plant from damage.

b. The SS is responsible for overall reactor operations, command in the control room and maintains the broadest perspective of operational conditions affecting the safety of the plant as a matter of highest priority at all times.

c. During emergency situations, the SS is in charge of the control room and should remain in the control room unless properly relieved.

d. The SS functions as Site Emergency Director and implements Emergency Plan procedures until relieved by a qualified higher management authority.

2. Unit Supervisor/Assistant Shift Supervisor:

The control room Unit Supervisor (SRO) reports to the SS and is in direct charge of operation of the assigned unit. The US is responsible for supervising, coordinating, and directing all activities to achieve safe, reliable, and efficient unit operation and taking necessary actions to ensure compliance with TS, operating license requirements, and approved plant procedures.

3. Shift Technical Advisor (STA)

The STA reports to the SS and is responsible for the following:

a. Providing an independent verification of critical safety functions, using redundant and diverse plant indications during transients and emergencies.

b. Immediately reporting any abnormalities or plant condition that may represent a challenge to the critical safety functions or that could result in a degradation of the safety level.

c. Assisting in evaluating the operability of plant equipment.

d. The STA may or may not be licensed (SRO). 4. Unit Operator/Reactor Operator

\section{Applicable Advanced Reactor System -}

\section{Applicable Knowledges/Skills/Abilities}

$\mathrm{K}-1$. Knowledge of system function, design and operation, including power

supplies and interlocks affecting system operation.

$\mathrm{K}-2$. Knowledge of the components in the system and the effects that a loss or malfunction of system component(s) would have on the performance of the system and on the operation of the system.

$\mathrm{K}-3$. Knowledge of the physical connections and/or cause-effect relations between the system and other systems, including knowledge of the effect that loss or malfunction of the system would have on other systems, and viceversa

K-4. Knowledge of procedures controlling normal, abnormal and emergency operations of the plant, and the contents and application of administrative procedures and the site Emergency Plan.

K-5. Knowledge of Technical Specifications and other regulatory requirements addressing operation and maintenance of the plant

(Ops-1) Monitor and evaluate plant status, and identify trends, using CR indications.

(Ops-2) Operate, using procedures, plant systems and equipment and monitor resulting changes to plant parameters and status

(Ops-3) Anticipate, recognize, monitor, verify, and report appropriately, automatic plant actuations and operations

(Ops-4) Determine/verify operability and availability of safety-related systems and equipment

(Ops-5) Monitor and cross-check diverse indications to verify and/or correctly understand plant conditions and to identify potential instrument or equipment malfunctions, and respond appropriately to malfunctions/failures using plant procedures

(OPS-6) Obtain, interpret and properly use CR reference materials, including normal operating procedures, EOPs, AOPs, E Plan, and drawings. (e.g., determine, using procedures, the anticipated effects on reactivity of plant

changes such as primary system cooldown or secondary system operations.)

(Ops-7) Respond appropriately to annunciator initiations, including silencing of distracting alarms, removal from service, and restoration, as well as responding to the indicated abnormality.

(Ops-8) Monitor, and operate appropriately from the CR, plant radiation control, release, instrumentation, and alarm systems using procedures and other administrative and regulatory guidance.

(OPS-9) Operate the plant computer to call up additional plant status and trend information beyond that available on panel instrumentations.

(OPS-10) Provide clear and complete shift turnover information, including panel walkdowns, to oncoming DR crewmembers providing plant status, conditions and operations in progress.

(Mgmt-1) Manage and lead the CR operating team.

(Mgmt-2) Manage and coordinate, using the plant communications system, operations tasks performed outside the $\mathrm{CR}$, and monitor $\mathrm{CR}$ indications of task performance.

(Mgmt-3) Provide clear and concise verbal communications to the $\mathrm{CR}$ operating crew, plant staff, supervisory personnel and management. (Mgmt-4) Write clear and accurate logs, records, and status tables documenting plant status and operator activities. 


\begin{tabular}{|c|c|c|c|}
\hline \multicolumn{3}{|c|}{$\begin{array}{l}\text { Lesson \# 624P-1.6 Title: Conduct Of Control Room Operations } \\
\text { Time: } 30 \text { Minutes }\end{array}$} & \multirow{2}{*}{$\begin{array}{l}\text { Applicable Advanced Reactor System - } \\
\text { Applicable Knowledges/Skills/Abilities } \\
\end{array}$} \\
\hline Objectives & Presenta & & \\
\hline & $\begin{array}{l}\text { Ops-3 } \\
\text { Ops-5 } \\
\text { Ops-7 } \\
\end{array}$ & 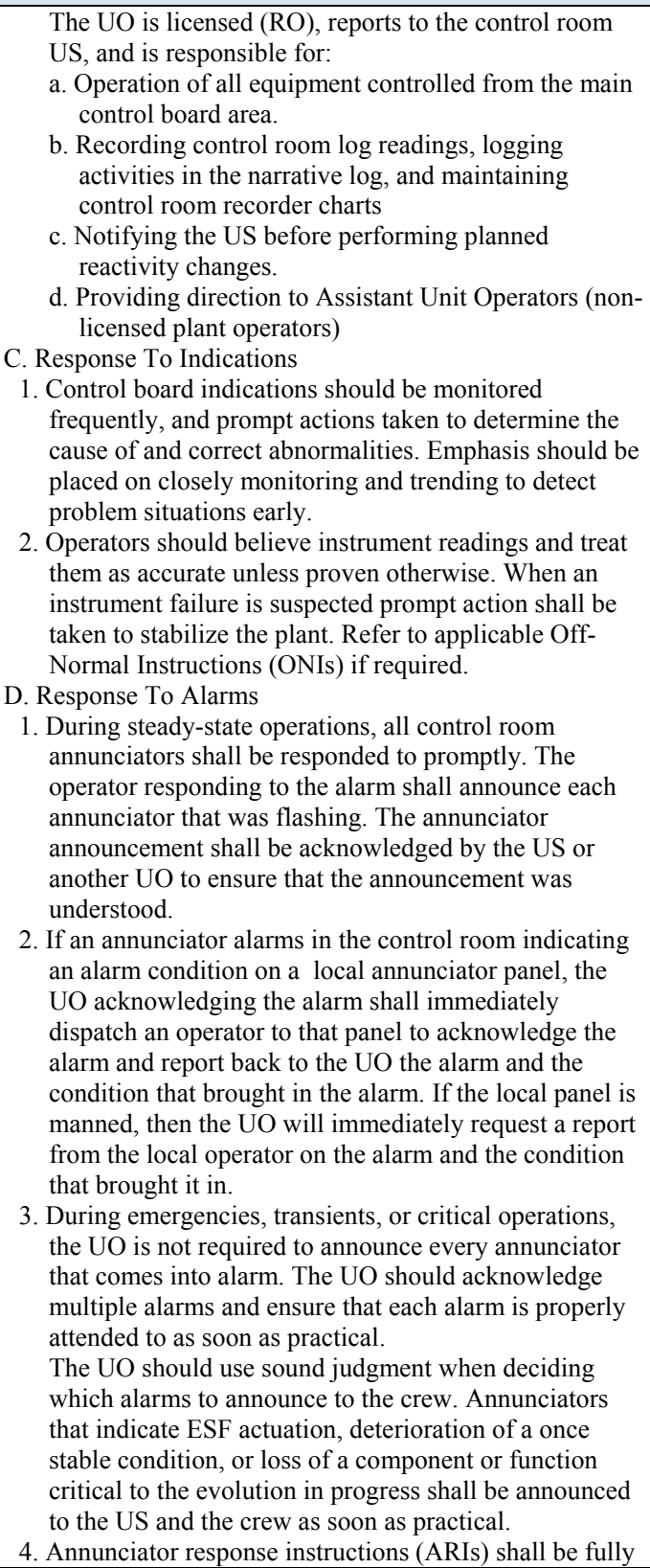 & $\begin{array}{l}\text { (Lic-1) Recognize entry conditions for EOPs, AOPs, TS Action Statements, } \\
\text { License Conditions, and Emergency Plan, and take appropriate actions. } \\
\text { (Lic-2) Document and track TS and other license-related issues and either } \\
\text { verify resolution prior to time-clock expiration or plan/initiate required plant } \\
\text { status changes. } \\
\text { (Lic-3) Determine the desired operational path forward based on plant status } \\
\text { information and administrative procedures and directives. }\end{array}$ \\
\hline
\end{tabular}




\begin{tabular}{|c|c|c|}
\hline \multicolumn{2}{|c|}{$\begin{array}{l}\text { Lesson \# 624P-1.6 Title: Conduct Of Control Room Operations } \\
\text { Time: } 30 \text { Minutes }\end{array}$} & Applicable Advanced Reactor System - \\
\hline Objectives & Presentation: & Applicable Knowledges/Skills/Abilities \\
\hline & \begin{tabular}{l|l} 
utilized by the operating crew. The US shall ensure \\
proper usage is maintained. If the UO knows that an \\
alarm was generated from a pre-planned activity in \\
progress, then the annunciator response procedures need \\
not be referenced. \\
E. Communications \\
1. Oral communications involving directives or parameters \\
that affect plant operation should be conducted using \\
the "three-way" communication technique. This \\
consists of the initial communication from the sender, \\
followed by a repeat back of the communication by the \\
receiver, and a confirmation of the receipt of the \\
communication by the sender. \\
2. When transmitting alpha-numeric information, the \\
phonetic alphabet listed in Attachment 2 or similar \\
should be used to ensure that letters are correctly \\
received. This is not necessary when referring to \\
standard approved acronyms such as RHR or CCW. \\
3. The completion of directed actions should be reported to \\
the governing station, normally the control room. \\
4. Written instructions or procedures should be provided \\
for complicated orders or other communications where \\
important information might be forgotten. For example, \\
extensive valving orders or extensive plant status \\
reports should be written.
\end{tabular} & \\
\hline
\end{tabular}


Table D.3. (contd)

\begin{tabular}{|c|c|c|c|}
\hline \multicolumn{3}{|c|}{$\begin{array}{l}\text { Lesson \# 624P-2.1 Title: System Review - CVCS, PZR Level , \& EHC } \\
\text { Time: } 60 \text { Minutes }\end{array}$} & Applicable AP1000 System - \\
\hline Objectives & Presentatio & & Applicable Knowledges/Skills/Abilities \\
\hline $\begin{array}{l}\text { 1.1 Provide the student with } \\
\text { the following detailed } \\
\text { information concerning the } \\
\text { CVCS, PZR Level , \& } \\
\text { EHC. } \\
\text { 1.1.1 Controls that may be } \\
\text { manipulated by the student } \\
\text { during the course of a } \\
\text { reactor startup and/or } \\
\text { power operations. } \\
\text { 1.1.2 Control switches that } \\
\text { must be operated to block } \\
\text { faulted instruments from } \\
\text { various automatic control } \\
\text { stations. } \\
\text { 1.1.3 Indications available to } \\
\text { the student from this } \\
\text { location used to evaluate } \\
\text { the status of the RCS } \\
\text { (CVCS and PZR level) or } \\
\text { the main turbine/generator } \\
\text { (EHC). }\end{array}$ & $\begin{array}{l}\text { K-1 } \\
\text { K-2 } \\
\text { K-3 } \\
\\
\text { Ops-7 } \\
\text { Ops-2 }\end{array}$ & $\begin{array}{l}\text { A. CVCS } \\
\text { 1. Review the CVCS with the diagram } \\
\text { a. Show major component locations on the control panel } \\
\text { b. Show the major instrumentation associated with CVCS } \\
\text { 2. Explain uses of back pressure regulator (PK- 131) } \\
\text { 3. Explain function of temperature divert valve (HIS-129) } \\
\text { 4. Explain the interlocks associated with the letdown } \\
\text { isolation valves (HIS- } 459 \text { and } 460 \text { ) and the letdown } \\
\text { orifice isolation valves (HIS- } 8149 \text { A, } 8149 \mathrm{~B}, \& \text { 8149C) } \\
\text { a. Cannot close } 459 \text { or } 460 \text { unless orifice valves are closed } \\
\text { b. Cannot open } 459 \text { or } 460 \text { unless orifice valves are closed } \\
\text { c. Cannot open } 8149 \text { A, B or C unless letdown isolation } \\
\text { valves are open } \\
\text { d. Cannot open either set of valves if pzr level }<17 \% \\
\text { 5. T.S. associated with CVCS } \\
\text { a. Boric acid and dilution flow paths } \\
\text { b. Centrifugal charging pumps } \\
\text { c. RCS specific activity and RCS Chemistry (indirect T.S. } \\
\text { due to flow through demineralizers) } \\
\text { B. CVCS Makeup } \\
\text { 1. Review makeup system with diagram } \\
\text { 2. Explain auto mode setup on MCB } \\
\text { a. Boric acid controller setpoint } \\
\text { b. Pure water controller internal setpoint } \\
\text { 3. Explain how to set up controllers for borations and } \\
\text { dilutions } \\
\text { a. Reset } \\
\text { b. Open windows and set in desired amounts } \\
\text { c. Start } \\
\text { C. Pressurizer Level Control } \\
\text { 1. Inputs into system } \\
\text { a. Auctioneered high Tavg } \\
\text { b. Selected pressurizer level } \\
\text { 2. Components controlled by system } \\
\text { a. Annunciators } \\
\text { b. Turn on backup heaters } \\
\text { c. CCP discharge flow control valve (FCV- 121) } \\
\text { d. PDP pump speed } \\
\text { e. Letdown isolation valves } \\
\text { f. Orifice isolation valves } \\
\text { g. } 17 \% \text { level heater interlock } \\
\text { 3. Control boards indications and controls } \\
\text { a. Level Indications } \\
\text { 1) Hot calibrated channels } \\
\text { 2) Cold calibrated channel } \\
\text { b. Recorder } \\
\text { c. CCP discharge flow control valve } \\
\text { d. PDP speed control }\end{array}$ & 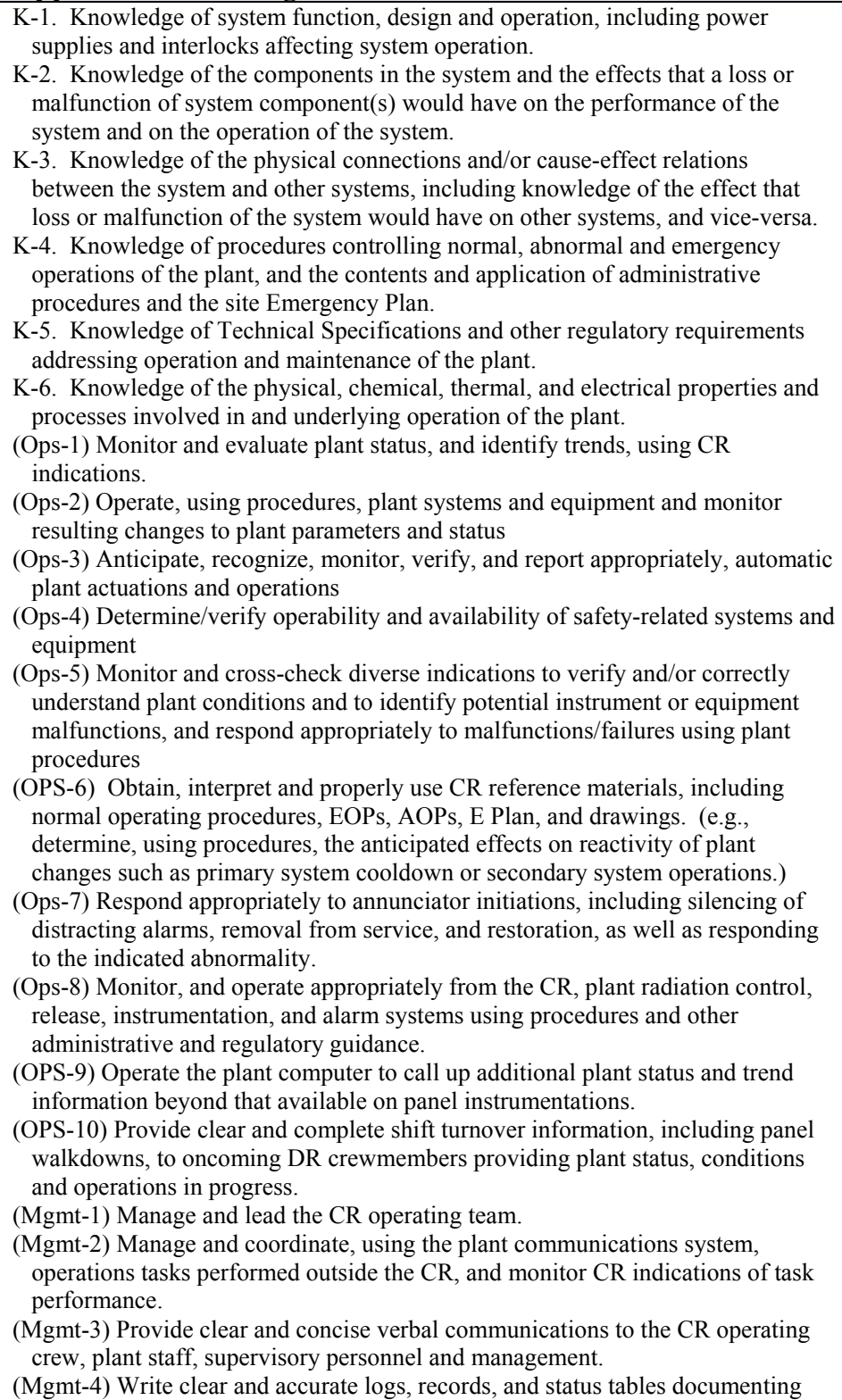 \\
\hline
\end{tabular}




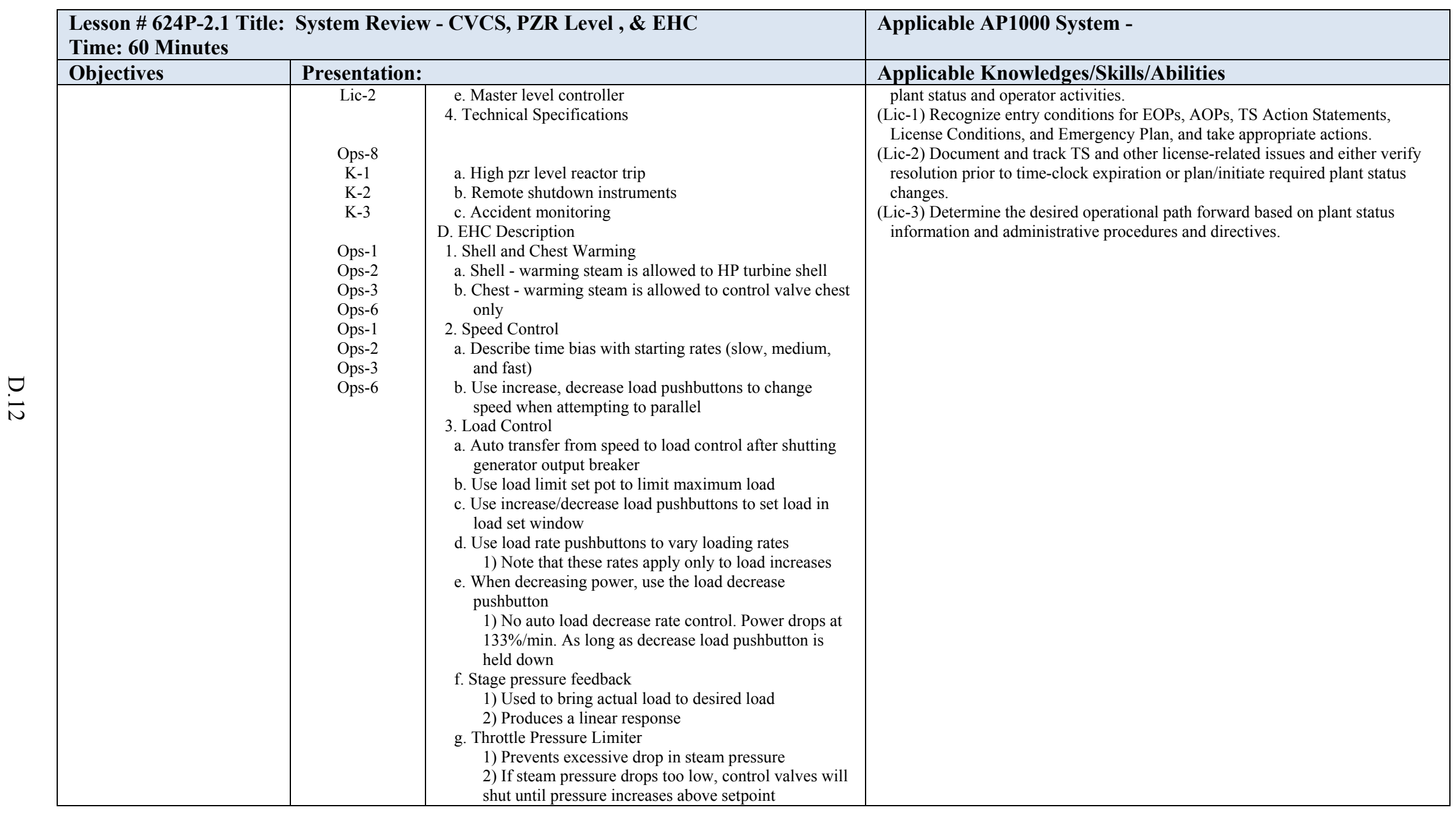


Table D.3. (contd)

\begin{tabular}{|c|c|c|c|}
\hline \multicolumn{3}{|c|}{$\begin{array}{l}\text { Lesson \# 624P-2.2 Title: Operations - Reactor Startup } \\
\text { Time: } 60 \text { Minutes }\end{array}$} & \multirow{2}{*}{$\begin{array}{l}\text { Applicable AP1000 System - } \\
\text { Applicable Knowledges/Skills/Abilities }\end{array}$} \\
\hline Objectives & Present & tion: & \\
\hline $\begin{array}{l}\text { 1.1 Provide the student with the } \\
\text { following: } \\
\text { 1.1.1 An understanding of how } \\
\text { the core is made critical. } \\
\text { 1.1.2 How to recognize } \\
\text { criticality. } \\
\text { 1.1.3 How to control the } \\
\text { reactivity of the core. } \\
\text { 1.1.4 Effects of subcritical } \\
\text { multiplication. } \\
\text { 1.1.5 Technical Specifications } \\
\text { that may be entered during } \\
\text { rod withdrawal. } \\
\\
\text { Simulator IC, MALF, LOA, or } \\
\text { TS Item } \\
\text { PLP RCS } 1 \\
\text { MALF NIS 9\} } \\
\text { FREEZE } \\
\text { \{TS 3.3.1\} } \\
\text { \{Table 3.3.1-1 item } 4 \text { \& 18\} }\end{array}$ & $\begin{array}{c}\text { Mgmt-1 } \\
\text { Mgmt-2 } \\
\text { Mgmt-3 } \\
\text { Mgmt-4 } \\
\text { K-5 } \\
\text { Lic-1 } \\
\text { Lic-2 } \\
\text { Lic-3 } \\
\text { Ops-2 }\end{array}$ & $\begin{array}{l}\text { A. After re-initializing remove } 15 \mathrm{ppm} \\
\text { boron from the RCS. } \\
\\
\text { B. Fail one IR channel } \\
\text { Channel }=35 \text { or } 36 \\
\text { Value }=1.015 \mathrm{E}-11 \\
\text { Ramp }=0 \\
\text { Delay }=0 \\
\text { C. Perform ECC } \\
\text { 1. Discuss reactivity balance. } \\
\text { 2. Explain same conditions as } \\
\text { previous day. } \\
\text { 3. No Xe, Sm, Fuel burnup changes. } \\
\text { 4. Only have to account for boron } \\
\text { change in RCS. } \\
\text { 5. Convert boron change to PCM 's } \\
\text { of reactivity/rod worth. } \\
\text { D. Perform Reactor Startup IAW GOI 2- } \\
\text { 2 } \\
\text { 1. When students discover failed IR, } \\
\text { FREEZE } \\
\text { 2. Discuss failed instrument } \\
\text { a. T.S. action is power dependent } \\
\text { b. Clear malfunction } \\
\text { 3. Continue reactor startup } \\
\text { E. Continue power escalation IAW GOI } \\
\text { 2-3 } \\
\text { F. Continue power escalation until all } \\
\text { control systems are in automatic. }\end{array}$ & 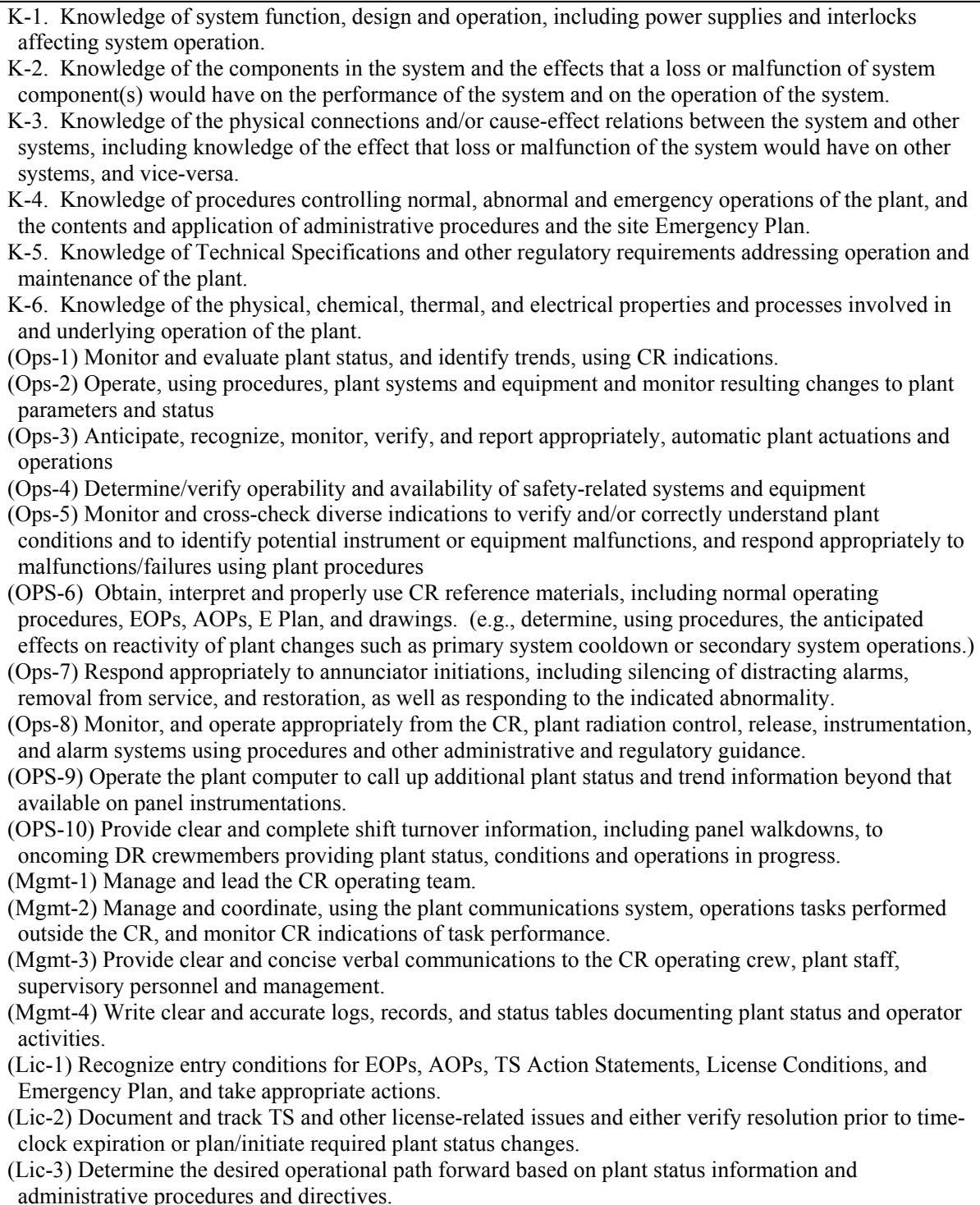 \\
\hline
\end{tabular}


Table D.3. (contd)

\begin{tabular}{|c|c|c|c|}
\hline \multicolumn{3}{|c|}{$\begin{array}{l}\text { Lesson \# 624P-2.3 Title: Operations - Power Maneuvering } \\
\text { Time: } 3 \text { Hours }\end{array}$} & \multirow{2}{*}{$\begin{array}{l}\text { Applicable AP1000 System - } \\
\text { Knowledges/Skills/Abilities }\end{array}$} \\
\hline Objectives & Presen & ation: & \\
\hline $\begin{array}{l}\text { 1.1 Provide the student with } \\
\text { the following: } \\
\text { 1.1.1 An understanding of } \\
\text { how power is } \\
\text { escalated }\end{array}$ & $\begin{array}{c}\text { Mgmt- } \\
1 \\
\text { Mgmt- } \\
2 \\
\text { Mgmy- } \\
3 \\
\text { Mgmt- } \\
4 \\
\text { Lic-1 } \\
\text { K-5 } \\
\text { Ops-2 } \\
\text { Ops-1 } \\
\\
\text { K-6 } \\
\\
\text { K-6 }\end{array}$ & $\begin{array}{l}\text { A. Perform a reactivity addition calculation } \\
\text { 1. Use boron change worksheet } \\
\text { 2. Use faber board to show calculation } \\
\text { 3. Use a } 30 \% \text { to } 75 \% \text { power change as an example } \\
\text { a. Explain power defect } \\
\text { 1) P adds - reactivity } \\
\text { 2) Must add + reactivity } \\
\text { b. Explain why rods full out is desired } \\
\text { 4. Explain rate of dilution } \\
\text { a. Max dilution depends upon charging rate } \\
\text { b. Max rate of power increase depends upon } \\
\text { dilution rate and maintaining delta flux } \\
\text { within its band } \\
\text { B. Initial Conditions } \\
\text { 1. Have shift supervisor perform a power to } 1165 \\
\text { MWe over the next } 2.5 \text { hours } \\
\text { 2. Tell students to follow all procedures and T.S. } \\
\text { actions as necessary } \\
\text { 3. Have shift supervisor read GOI } 5 \text { paragraph } \\
\text { 4.12 thru } 4.14 \\
\text { a. Explain that plants would have the fuel } \\
\text { preconditioning limits but because of } \\
\text { training time constraints will be } \\
\text { disregarded } \\
\text { C. Power to full load IAW GOI } 5 \\
\text { 1. Perform calorimetric at } 50 \% \\
\text { a. Use calorimetric worksheets } \\
\text { b. Explain that the calorimetric is performed } \\
\text { to ensure that the NIs are calibrated to } \\
\text { indicate thermal power } \\
\text { c. Explain RCP and S/G blowdown } \\
\text { correction factors that are applied to the } \\
\text { calculation. } \% \\
\text { 2. At approximately } 60 \% \text { power, fail PZR level } \\
\text { channel } 459 \\
\text { a. Set } \\
\text { 1) Channel }=459 \\
\text { 2) Level = Low } \\
\text { 3) Delay = } 180 \text { seconds } \\
\text { b. Activate } \\
\text { 3. Go to freeze after students have selected out the } \\
\text { failed channel } \\
\text { a. Discuss the failed channel } \\
\text { b. Analog channel check required at least once } \\
\text { per } 12 \text { hours } \\
\text { c. Discuss procedures to be followed } \\
\text { 4. Continue power to } 100 \% \\
\text { and }\end{array}$ & 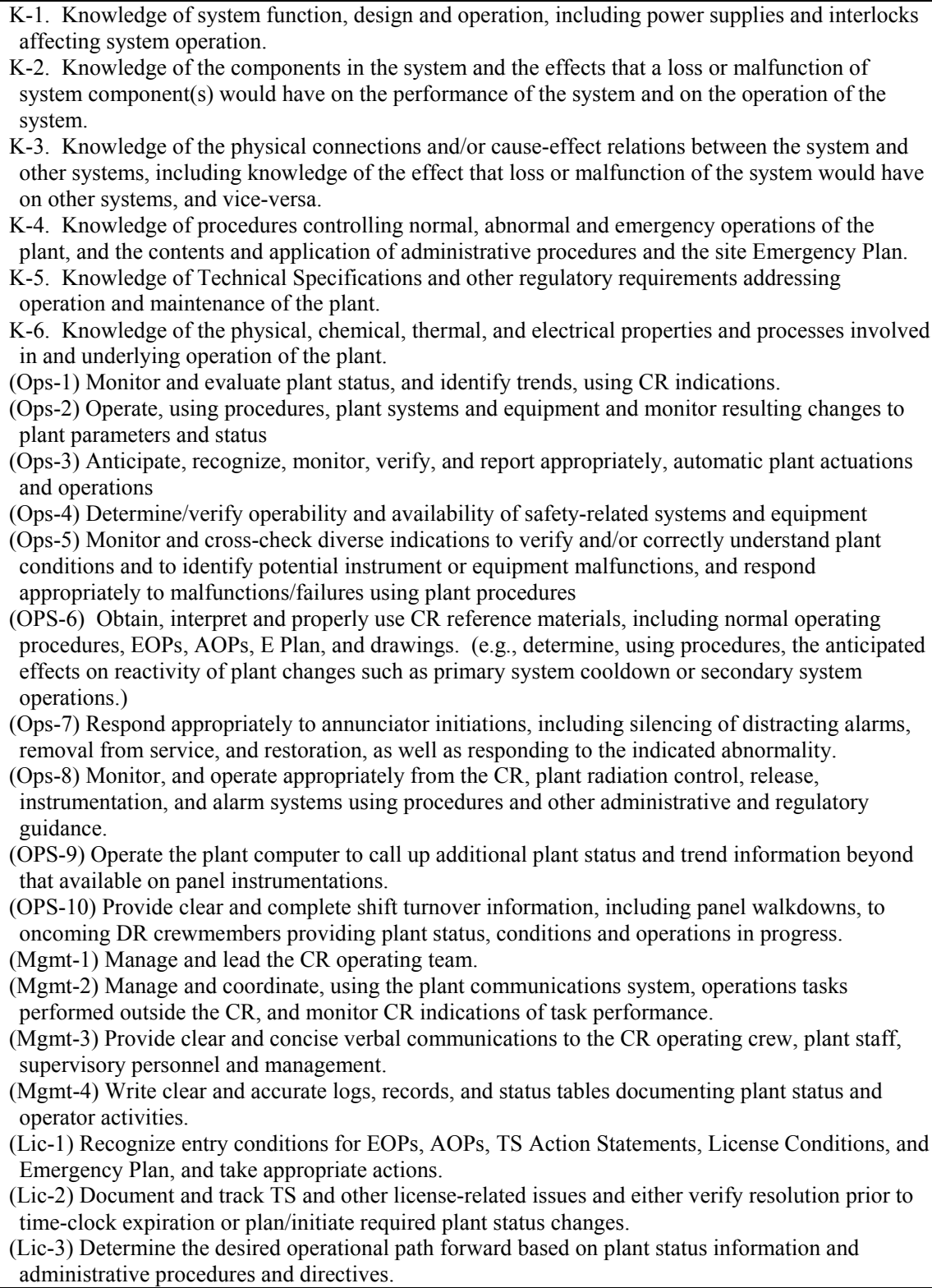 \\
\hline
\end{tabular}


Table D.3. (contd)

\begin{tabular}{|c|c|c|c|}
\hline \multicolumn{3}{|c|}{$\begin{array}{l}\text { Lesson \# 624P-2.4 Title: System Review - Main Steam \& Steam } \\
\text { Dump Control } \\
\text { Time: } 60 \text { Minutes }\end{array}$} & Applicable AP1000 System - \\
\hline Objectives & Presen & tion: & Knowledges/Skills/Abilities \\
\hline $\begin{array}{l}\text { 1.1 Provide the student with the } \\
\text { following detailed } \\
\text { information concerning the } \\
\text { Main Steam and Steam Dump } \\
\text { Control Systems: } \\
\text { 1.1.1 Controls that may be } \\
\text { manipulated by the student } \\
\text { during the course of a } \\
\text { reactor startup and/or } \\
\text { power operations. } \\
\text { 1.1.2 Controllers that must be } \\
\text { manipulated during power } \\
\text { operations. } \\
\text { 1.1.3 Indications available to the } \\
\text { student from this location } \\
\text { used to evaluate the } \\
\text { status of the secondary } \\
\text { plant. } \\
\text { Simulator IC, MALF, LOA, or } \\
\text { TS Item } \\
\text { INIT } 16 \\
\text { Panel C } 02 \\
\text { Panel C } 14 / 15 \\
\text { Panel C } 14 \text { \& C } 5 \\
\text { Panel C } 14 \\
\text { Panel C } 14 \\
\text { Panel C } 15 \\
\text { Panel C } 15\end{array}$ & $\begin{array}{c} \\
\mathrm{K}-2 \\
\mathrm{~K}-3 \\
\text { Ops-2 }\end{array}$ & $\begin{array}{l}\text { A. System Description } \\
\text { 1. Main Steam } \\
\text { a. Use the steam system diagrams } \\
\text { to show the flow path of the } \\
\text { steam from the S/G to the major } \\
\text { steam loads } \\
\text { B. Controls } \\
\text { 1. Show the location and explain } \\
\text { how to use the following: } \\
\text { a. Main steam isolation valves } \\
\text { b. Steam pressure controller PK- } \\
\text { 507 } \\
\text { 1) Explain how the setpoint for } \\
\text { the pot is derived } \\
\text { c. Steam dump mode selector } \\
\text { switch } \\
\text { d. Steam dump interlock switches } \\
\text { e. Main steam isolation bypass } \\
\text { valves } \\
\text { f. S/G atmospheric relief valves } \\
\text { 1) Describe both the manual and } \\
\text { automatic operation of these } \\
\text { controllers } \\
\text { C. Instrumentation } \\
\text { 1. Show the location and explain } \\
\text { the use of the following: } \\
\text { a. Steam flow indicators } \\
\text { b. Condenser back pressure } \\
\text { c. Main steam header pressure } \\
\text { d. Steam dump valve position } \\
\text { e. Steam dump demanded position }\end{array}$ & 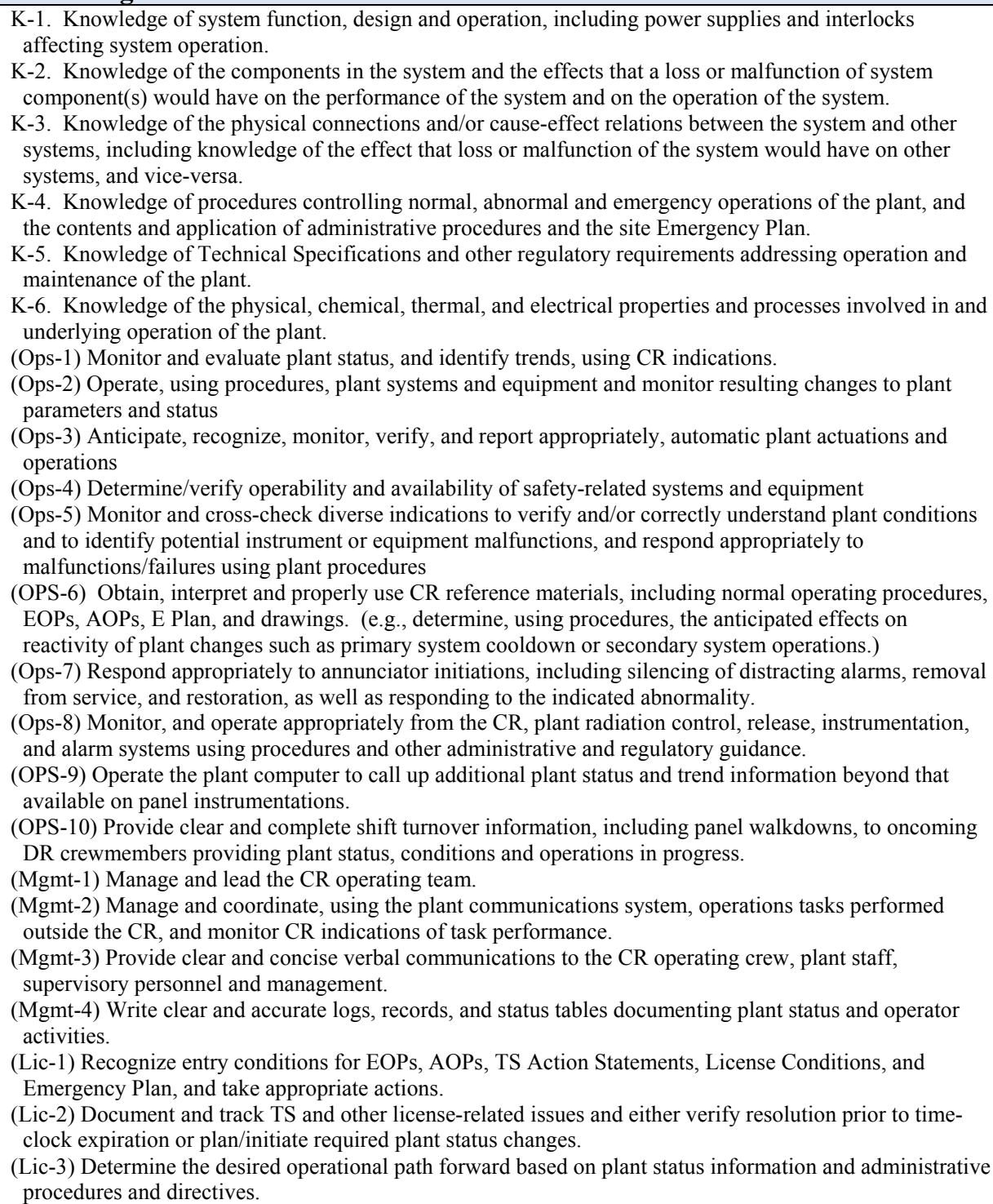 \\
\hline
\end{tabular}


Table D.4. Task/KSA Inventory Items and Elements of Control Room Fidelity: Expert Panel Rating

\begin{tabular}{|c|c|c|c|c|c|c|c|c|c|}
\hline \multirow[t]{2}{*}{ Control Room Tasks Taxonomy } & \multicolumn{7}{|c|}{ Elements of Control Room Fidelity } & \multicolumn{2}{|c|}{ Assumed Technology } \\
\hline & N/A & Displays & $\begin{array}{l}\text { Operator } \\
\text { Input } \\
\text { Devices } \\
\end{array}$ & Alarms & $\begin{array}{l}\text { Decision } \\
\text { Aides }\end{array}$ & $\begin{array}{c}\text { Communication } \\
\text { Equipment }\end{array}$ & $\begin{array}{l}\text { Workplace } \\
\text { Design }\end{array}$ & $\begin{array}{l}\text { Simulator } \\
\text { Model }\end{array}$ & $\begin{array}{l}\text { User - } \\
\text { System } \\
\text { Interaction }\end{array}$ \\
\hline $\begin{array}{l}\text { (K-1) Knowledge of system function, design and } \\
\text { operation, including power supplies and } \\
\text { interlocks affecting system operation. }\end{array}$ & $\mathrm{X}$ & XX & $\mathbf{X}$ & & $\mathrm{X}$ & & $\mathrm{X}$ & & \\
\hline $\begin{array}{l}\text { (K-2) Knowledge of the components in the system } \\
\text { and the effects that a loss or malfunction of } \\
\text { system component(s) would have on the } \\
\text { performance of the system and on the operation } \\
\text { of the system. }\end{array}$ & & $\mathbf{X}$ & $X$ & $\mathbf{X X}$ & $\mathbf{X}$ & & & $\mathbf{X}$ & \\
\hline $\begin{array}{l}\text { (K-3) Knowledge of the physical connections and/or } \\
\text { cause-effect relations between the system and } \\
\text { other systems, including knowledge of the effect } \\
\text { that loss or malfunction of the system would } \\
\text { have on other systems, and vice-versa. }\end{array}$ & & $\mathbf{X X}$ & & & $\mathbf{X X}$ & & & $\mathbf{X X}$ & \\
\hline $\begin{array}{l}\text { (K-4) Knowledge of procedures controlling normal, } \\
\text { abnormal and emergency operations of the } \\
\text { plant, and the contents and application of } \\
\text { administrative procedures and the site } \\
\text { Emergency Plan. }\end{array}$ & & & & & $\mathbf{X X X X X X X}$ & & & & \\
\hline $\begin{array}{l}\text { (K-5) Knowledge of Technical Specifications and } \\
\text { other regulatory requirements addressing } \\
\text { operation and maintenance of the plant. }\end{array}$ & & & & & $\mathbf{X X X X X X X}$ & & & & \\
\hline $\begin{array}{l}\text { (K-6) Knowledge of the physical, chemical, } \\
\text { thermal, and electrical properties and processes } \\
\text { involved in and underlying operation of the } \\
\text { plant. }\end{array}$ & & & & & $\mathbf{X X X X X}$ & & $\mathbf{X}$ & $\mathbf{X}$ & \\
\hline $\begin{array}{l}\text { (Ops-1) Monitor and evaluate plant status, and } \\
\text { identify trends, using CR indications. }\end{array}$ & & $\mathbf{X X X X X X X}$ & & & & & & & \\
\hline $\begin{array}{l}\text { (Ops-2) Operate, using procedures, plant systems } \\
\text { and equipment and monitor resulting changes to } \\
\text { plant parameters and status. }\end{array}$ & & $X \mathbf{X}$ & $\mathbf{X X X X X}$ & & $\mathrm{X}$ & & & & \\
\hline $\begin{array}{l}\text { (Ops-3) Anticipate, recognize, monitor, verify, and } \\
\text { report appropriately, automatic plant actuations } \\
\text { and operations. }\end{array}$ & & $\mathbf{X X X X X X}$ & & $\mathbf{X X}$ & & & & & \\
\hline $\begin{array}{l}\text { (Ops-4) Determine/verify operability and } \\
\text { availability of safety-related systems and } \\
\text { equipment }\end{array}$ & & $\mathbf{X X X X X X}$ & $\mathbf{X}$ & & & & & & \\
\hline
\end{tabular}




\begin{tabular}{|c|c|c|c|c|c|c|c|c|c|}
\hline \multirow[t]{2}{*}{ Control Room Tasks Taxonomy } & \multicolumn{7}{|c|}{ Elements of Control Room Fidelity } & \multicolumn{2}{|c|}{ Assumed Technology } \\
\hline & N/A & Displays & $\begin{array}{l}\text { Operator } \\
\text { Input } \\
\text { Devices } \\
\end{array}$ & Alarms & $\begin{array}{c}\text { Decision } \\
\text { Aides }\end{array}$ & $\begin{array}{c}\text { Communication } \\
\text { Equipment }\end{array}$ & $\begin{array}{l}\text { Workplace } \\
\text { Design }\end{array}$ & $\begin{array}{c}\text { Simulator } \\
\text { Model }\end{array}$ & $\begin{array}{l}\text { User - } \\
\text { System } \\
\text { Interaction }\end{array}$ \\
\hline $\begin{array}{l}\text { (Ops-5) Monitor and cross-check diverse } \\
\text { indications to verify and/or correctly understand } \\
\text { plant conditions and to identify potential } \\
\text { instrument or equipment malfunctions, and } \\
\text { respond appropriately to malfunctions/failures } \\
\text { using plant procedures. }\end{array}$ & & $\mathbf{X X X X}$ & $\mathbf{X}$ & $\mathbf{X X}$ & $\mathbf{X}$ & & & & \\
\hline $\begin{array}{l}\text { (Ops-6) Obtain, interpret and properly use CR } \\
\text { reference materials, including normal operating } \\
\text { procedures, EOPs, AOPs, E Plan, and drawings. } \\
\text { (e.g., determine, using procedures, the } \\
\text { anticipated effects on reactivity of plant changes } \\
\text { such as primary system cooldown or secondary } \\
\text { system operations.) }\end{array}$ & & & $\mathrm{X}$ & & $\mathbf{X X X X X X}$ & & & & \\
\hline $\begin{array}{l}\text { Ops-7) Respond appropriately to annunciator } \\
\text { initiations, including silencing of distracting } \\
\text { alarms, removal from service, and restoration, as } \\
\text { well as responding to the indicated abnormality. }\end{array}$ & & $\mathrm{X}$ & $\mathbf{X X}$ & $\mathbf{X X X X}$ & & & & & \\
\hline $\begin{array}{l}\text { (Ops-8) Monitor, and operate appropriately from the } \\
\text { CR, plant radiation control, release, } \\
\text { instrumentation, and alarm systems using } \\
\text { procedures and other administrative and } \\
\text { regulatory guidance. }\end{array}$ & & $\mathbf{X}$ & $\mathbf{X X X X X}$ & & $\mathrm{X}$ & & & & \\
\hline $\begin{array}{l}\text { (Ops-9) Operate the plant computer to call up } \\
\text { additional plant status and trend information } \\
\text { beyond that available on panel instrumentations. }\end{array}$ & & & $\mathbf{X}$ & & $\mathbf{X X X} \times \mathbf{X}$ & & $\mathbf{X}$ & & \\
\hline $\begin{array}{l}\text { (Ops-10) Provide clear and complete shift turnover } \\
\text { information, including panel walkdowns, to } \\
\text { oncoming DR crewmembers providing plant } \\
\text { status, conditions and operations in progress. }\end{array}$ & & $\mathbf{X X X}$ & & & $\mathbf{X}$ & & $\mathbf{X X X}$ & & \\
\hline (Mgmt-1) Manage and lead the CR operating team. & & $\mathbf{X X}$ & & & $\mathbf{X}$ & & $\mathbf{X X X}$ & & \\
\hline $\begin{array}{l}\text { (Mgmt-2) Manage and coordinate, using the plant } \\
\text { communications system, operations tasks } \\
\text { performed outside the CR, and monitor CR } \\
\text { indications of task performance. }\end{array}$ & & $\mathrm{X}$ & & & & $\mathbf{X X X X X}$ & & & \\
\hline $\begin{array}{l}\text { Mgmt-3) Provide clear and concise verbal } \\
\text { communications to the CR operating crew, plant } \\
\text { staff, supervisory personnel and management. }\end{array}$ & & & & & $\mathbf{X X}$ & $\mathbf{X X}$ & $\mathbf{X X X}$ & & \\
\hline $\begin{array}{l}\text { (Mgmt-4) Write clear and accurate logs, records, } \\
\text { and status tables documenting plant status and } \\
\text { operator activities. }\end{array}$ & & & $\mathrm{X}$ & & $\mathbf{X X X}$ & & $\mathbf{X X X}$ & & \\
\hline
\end{tabular}




\begin{tabular}{|c|c|c|c|c|c|c|c|c|c|}
\hline \multirow[t]{2}{*}{ Control Room Tasks Taxonomy } & \multicolumn{7}{|c|}{ Elements of Control Room Fidelity } & \multicolumn{2}{|c|}{ Assumed Technology } \\
\hline & N/A & Displays & $\begin{array}{l}\text { Operator } \\
\text { Input } \\
\text { Devices }\end{array}$ & Alarms & $\begin{array}{c}\text { Decision } \\
\text { Aides }\end{array}$ & $\begin{array}{c}\text { Communication } \\
\text { Equipment }\end{array}$ & $\begin{array}{l}\text { Workplace } \\
\text { Design }\end{array}$ & $\begin{array}{c}\text { Simulator } \\
\text { Model }\end{array}$ & $\begin{array}{l}\text { User - } \\
\text { System } \\
\text { Interaction }\end{array}$ \\
\hline $\begin{array}{l}\text { (Lic-1) Recognize entry conditions for EOPs, } \\
\text { AOPs, TS Action Statements, License } \\
\text { Conditions, and Emergency Plan, and take } \\
\text { appropriate actions. }\end{array}$ & & $\mathbf{X X X}$ & $\mathbf{X}$ & $\mathrm{X}$ & $\mathbf{X}$ & & & & \\
\hline $\begin{array}{l}\text { (Lic-2) Document and track TS and other license- } \\
\text { related issues and either verify resolution prior } \\
\text { to time-clock expiration or plan/initiate required } \\
\text { plant status changes. }\end{array}$ & & & $\mathrm{X}$ & & $\mathbf{X X X X X X}$ & & & & \\
\hline $\begin{array}{l}\text { (Lic-3) Determine the desired operational path } \\
\text { forward based on plant status information and } \\
\text { administrative procedures and directives. }\end{array}$ & & $\mathbf{X}$ & & & $\mathbf{X X X X X X}$ & & & & \\
\hline
\end{tabular}

Reviewer 1 - Black; Reviewer 2 -Red; Reviewer 3 - Blue; Reviewer 4 - Green; Reviewer 5 - Yellow; Reviewer 6 - Purple; Reviewer 7 - Orange 
Appendix E

The On-Line Survey 


\section{Appendix E}

\section{The On-Line Survey}

\section{Appendix E.1 On-line Survey: Resident Inspectors \\ Reformatted for print version}

In preparation for training NRC inspectors and license examiners for next generation reactors (e.g., AP-1000, ABWR, EPR, and ESBWR) the NRC has commissioned Pacific Northwest National Laboratory to characterize TTC simulator needs, especially with regard to physical fidelity. Physical fidelity is how closely the look, feel, sound, layout, location, and operator interface of a training simulator matches an actual control room or site-specific control room simulator. This questionnaire seeks your expert input on your experience regarding:

- The most significant job-related differences in physical fidelity between a TTC simulator you trained on and the control room of the plant to which you were then assigned.

- How these differences affected your learning the necessary job-related skills and knowledge during TTC simulator training.

- How these differences affected your ability to apply or transfer your TTC simulator training to your plant.

- How your training addressed these differences.

This questionnaire also seeks your assessment of the TTC simulator training you received.

This questionnaire is specifically for Resident Inspectors only. If you are not a Resident Inspector, please do not complete this questionnaire.

For the questions In this survey please refer to your current job position at your currently assigned plant and the TTC training you received for it, unless instructed otherwise.

${ }^{*}$ Questions with a red asterisk are required.

A Note on Privacy Unless you voluntarily provide your name or telephone number for follow up questions by project staff or potential participation in a focus group, this survey is anonymous and the record kept of your survey responses does not contain any identifying information about you. Aggregated survey results may be shared publicly. Such aggregated results will contain no personally identifying information. 


\section{Effect of Differences in Physical Fidelity}

For each major control room attribute listed you are asked to assess the effect of physical fidelity differences between a TTC training simulator and the control room of the plant at which you are assigned and to characterize the most significant job-related differences. Please consider the TTC training you have received associated with your current job position only.

You will be assessing the following control room simulator attributes:

- Panels

- Controllers and Switches

- Instrumentation

- Alarms/Annunciators

- Computer Displays

- Procedures

- Control Room Environment

\section{Refresher Training}

1. Have you received TTC refresher simulator training relevant for the control room at the plant to which you are currently assigned? * (simple page jumping)

O Yes; O No

\section{Effect of Differences in Panels (Refresher)}

Please consider the TTC simulator training you have received associated with your current job position at your currently assigned plant only.

2. In terms of physical fidelity, how different are the PANELS in the TTC simulator from the control room at your assigned plant? Example differences may include the layout, location, and existence of PANELS. * (show/hide trigger question)

\begin{tabular}{|l|l|l|l|l|l|l|}
\hline & 1 & 2 & 3 & 4 & 5 & \\
No Difference & $\mathrm{O}$ & $\mathrm{O}$ & $\mathrm{O}$ & $\mathrm{O}$ & $\mathrm{O}$ & Entirely Different \\
\hline
\end{tabular}

3. What impact did these differences in PANELS have on your ability to perform your job duties when you first arrived at your assigned plant after your initial TTC simulator training? *

\begin{tabular}{|c|c|c|c|c|c|c|}
\hline & 1 & 2 & 3 & 4 & 5 & High Impact \\
\hline
\end{tabular}

4. What impact did these differences in PANELS have on how difficult it was for you to learn course lessons during the refresher training? *

\begin{tabular}{|c|c|c|c|c|c|c|}
\hline & 1 & 2 & 3 & 4 & 5 & High Impact \\
\hline
\end{tabular}

5. Please describe the differences in PANELS that had the most significant effect on your ability to perform your job duties after your initial simulator training or learn course lessons during refresher training:

[Space for response text] 


\section{Effect of Differences in Controllers/Switches (Refresher)}

Please consider the TTC simulator training you have received associated with your current job position at your currently assigned plant only.

6. In terms of physical fidelity, how different are the CONTROLLER/SWITCHES in the TTC simulator from the control room at your assigned plant? Example differences may include the look, feel, layout, location, and existence of CONTROLLER/SWITCHES. * (show/hide trigger question)

\begin{tabular}{|l|l|l|l|l|l|l|}
\hline & 1 & 2 & 3 & 4 & 5 & Entirely Different \\
\hline
\end{tabular}

7. What impact did these differences in CONTROLLERS/SWITCHES have on your ability to perform your job duties when you first arrived at your assigned plant after your initial TTC simulator training? *

\begin{tabular}{|c|c|c|c|c|c|c|}
\hline & 1 & 2 & 3 & 4 & 5 & High Impact \\
\hline
\end{tabular}

8. What impact did these differences in CONTROLLERS/SWITCHES have on how difficult it was for you to learn course lessons during the refresher training? *

\begin{tabular}{|c|c|c|c|c|c|c|}
\hline & 1 & 2 & 3 & 4 & 5 & High Impact \\
\hline
\end{tabular}

9. Please describe the differences in CONTROLLERS/SWITCHES that had the most significant effect on your ability to perform your job duties after your initial simulator training or learn course lessons during refresher training:

[Space for response text]

\section{Effect of Differences in Instrumentation (Refresher)}

Please consider the TTC simulator training you have received associated with your current job position at your currently assigned plant only.

10. In terms of physical fidelity, how different is the INSTRUMENTATION in the TTC simulator from the control room at your assigned plant r? Example differences may include the look, sound, layout, location, and existence of INSTRUMENTATION. * (show/hide trigger question)

\begin{tabular}{|c|c|c|c|c|c|c|}
\hline & 1 & 2 & 3 & 4 & 5 & Entirely Different \\
\hline
\end{tabular}

11. What impact did these differences in INSTRUMENTATION have on your ability to perform your job duties when you first arrived at your assigned plant after your initial TTC simulator training? *

\begin{tabular}{|c|c|c|c|c|c|c|}
\hline & 1 & 2 & 3 & 4 & 5 & High Impact \\
\hline
\end{tabular}


12. What impact did these differences in INSTRUMENTATION have on how difficult it was for you to learn course lessons during the refresher training? *

\begin{tabular}{|c|c|c|c|c|c|c|}
\hline & 1 & 2 & 3 & 4 & 5 & High Impact \\
\hline
\end{tabular}

13. Please describe the differences in INSTRUMENTATION that had the most significant effect on your ability to perform your job duties after your initial simulator training or learn course lessons during refresher training:

[Space for response text]

\section{Effect of Differences in Alarms/ Annunciators (Refresher)}

Please consider the TTC simulator training you have received associated with your current job position at your currently assigned plant only.

14. In terms of physical fidelity, how different are the ALARMS/ANNUNCIATORS in the TTC simulator from the control room at your assigned plant? Example differences may include the look, sound, layout, location, equipment/presentation medium, and existence of ALARMS/ANNUNCIATORS. * (show/hide trigger question)

\begin{tabular}{|l|l|l|l|l|l|l|}
\hline & 1 & 2 & 3 & 4 & 5 & \\
No Difference & 0 & 0 & 0 & 0 & 0 & Entirely Different \\
\hline
\end{tabular}

15. What impact did these differences in ALARMS/ANNUNCIATORS have on your ability to perform your job duties when you first arrived at at your assigned plant after your initial TTC simulator training? *

\begin{tabular}{|c|c|c|c|c|c|c|}
\hline & 1 & 2 & 3 & 4 & 5 & High Impact \\
\hline
\end{tabular}

16. What impact did these differences in ALARMS/ANNUNCIATORS have on how difficult it was for you to learn course lessons during the refresher training? *

\begin{tabular}{|c|c|c|c|c|c|c|}
\hline & 1 & 2 & 3 & 4 & 5 & High Impact \\
\hline
\end{tabular}

17. Please describe the differences in ALARMSIANNUNCIATORS that had the most significant effect on your ability to perform your job duties after your initial simulator training or learn course lessons during refresher training:

[Space for response text] 


\section{Effect of Differences in Displays (Refresher)}

Please consider the TTC simulator training you have received associated with your current job position at your currently assigned plant only.

18. In terms of physical fidelity, how different are the COMPUTER DISPLAYS in the TTC simulator from the control room at your assigned plant? Example differences may include the look, layout, location, and existence of COMPUTER DISPLAYS. * (show/hide trigger question

\begin{tabular}{|l|l|l|l|l|l|l|}
\hline & 1 & 2 & 3 & 4 & 5 & \\
No Difference & 0 & 0 & 0 & 0 & 0 & Entirely Different \\
\hline
\end{tabular}

19. What impact did these differences in COMPUTER DISPLAYS have on your ability to perform your job duties when you first arrived at your assigned plant after your initial TTC simulator training? *

\begin{tabular}{|c|c|c|c|c|c|c|}
\hline & 1 & 2 & 3 & 4 & 5 & High Impact \\
\hline
\end{tabular}

20. What impact did these differences in COMPUTER DISPLAYS have on how difficult it was for you to learn course lessons during the refresher training? *

\begin{tabular}{|c|c|c|c|c|c|c|}
\hline & 1 & 2 & 3 & 4 & 5 & High Impact \\
\hline
\end{tabular}

21. Please describe the differences in COMPUTER DISPLAYS that had the most significant effect on your ability to perform your job duties after your initial simulator training or learn course lessons during refresher training:

[Space for response text]

\section{Effect of Differences in Procedures (Refresher)} Please consider the TTC simulator training you have received associated with your current job position at your
currently assigned plant only.

22. In terms of physical fidelity, how different are the PROCEDURES in the TTC simulator from the control room at your assigned plant? Example differences may include the look, layout/organization, storage locations, and equipment/presentation medium of PROCEDURES. Please do not consider differences in Procedure content. * (show/hide trigger question)

\begin{tabular}{|l|l|l|l|l|l|l|}
\hline & 1 & 2 & 3 & 4 & 5 & Entirely Different \\
\hline
\end{tabular}

23. What impact did these differences in PROCEDURES have on your ability to perform your job duties when you first arrived at at your assigned plant after your initial TTC simulator training? * (hidden).

\begin{tabular}{|c|c|c|c|c|c|c|}
\hline & 1 & 2 & 3 & 4 & 5 & High Impact \\
\hline
\end{tabular}


24. What impact did these differences in PROCEDURES have on how difficult it was for you to learn course lessons during the refresher training? *

\begin{tabular}{|c|c|c|c|c|c|c|}
\hline & 1 & 2 & 3 & 4 & 5 & High Impact \\
\hline
\end{tabular}

25. Please describe the differences in PROCEDURES that had the most significant effect on your ability to perform your job duties after your initial simulator training or learn course lessons during refresher training. Please do not consider differences in Procedure content.

[Space for response text]

\section{Effect of Differences in Control Room Environment (Refresher)}

Please consider the TTC simulator training you have received associated with your current job position at your currently assigned plant only.

26. In terms of physical fidelity, how different is the CONTROL ROOM ENVIRONMENT in the TTC simulator from the control room at your assigned plant? Example differences may include the look, lighting, sound/ambient noise, and layout of the CONTROL ROOM ENVIRONMENT. * (show/hide trigger question)

\begin{tabular}{|l|l|l|l|l|l|l|}
\hline & 1 & 2 & 3 & 4 & 5 & Entirely Different \\
\hline
\end{tabular}

27. What impact did these differences in the CONTROL ROOM ENVIRONMENT have on your ability to perform your job duties when you first arrived at your assigned plant after your initial TTC simulator training? *

\begin{tabular}{|c|c|c|c|c|c|c|}
\hline & 1 & 2 & 3 & 4 & 5 & High Impact \\
\hline
\end{tabular}

28. What impact did these differences in the CONTROL ROOM ENVIRONMENT have on how difficult it was for you to learn course lessons during the refresher training? *

\begin{tabular}{|c|c|c|c|c|c|c|}
\hline & 1 & 2 & 3 & 4 & 5 & High Impact \\
\hline
\end{tabular}

29. Please describe the differences in CONTROL ROOM ENVIRONMENT that had the most significant effect on your ability to perform your job duties after your initial simulator training or learn course lessons during refresher training:

[Space for response text] 


\section{Effect of Differences in Panels (Non-refresher)}

Please consider the TTC simulator training you have received associated with your current job position at your currently assigned plant only.

30. In terms of physical fidelity, how different are the PANELS in the TTC simulator from the control room at your assigned plant? Example differences may include the layout, location, and existence of PANELS. * (show/hide trigger question)

\begin{tabular}{|l|l|l|l|l|l|l|}
\hline & 1 & 2 & 3 & 4 & 5 & Entirely Different \\
\hline
\end{tabular}

31. What impact did these differences in PANELS have on your ability to perform your job duties when you first arrived at your assigned plant after your initial TTC simulator training? *

\begin{tabular}{|c|c|c|c|c|c|c|}
\hline & 1 & 2 & 3 & 4 & 5 & High Impact \\
\hline
\end{tabular}

32. Please describe the differences in PANELS that had the most significant effect on your ability to perform your job duties after your initial simulator training:

[Space for response text]

\section{Effect of Differences in Controllers/Switches (Non-refresher)}

Please consider the TTC simulator training you have received associated with your current job position at your currently assigned plant only.

33. In terms of physical fidelity, how different are the CONTROLLER/SWITCHES in the TTC simulator from the control room at your assigned plant? Example differences may include the look, feel, layout, location, and existence of CONTROLLER/SWITCHES. * (show/hide trigger question)

\begin{tabular}{|l|l|l|l|l|l|l|}
\hline & 1 & 2 & 3 & 4 & 5 & Entirely Different \\
\hline
\end{tabular}

34. What impact did these differences in CONTROLLERS/SWITCHES have on your ability to perform your job duties when you first arrived at your assigned plant after your initial TTC simulator training? *

\begin{tabular}{|c|c|c|c|c|c|c|}
\hline & 1 & 2 & 3 & 4 & 5 & High Impact \\
\hline
\end{tabular}

35. Please describe the differences in CONTROLLERS/SWITCHES that had the most significant effect on your ability to perform your job duties after your initial simulator training:

[Space for response text] 


\section{Effect of Differences in Instrumentation (Non-refresher)}

Please consider the TTC simulator training you have received associated with your current job position at your currently assigned plant only.

36. In terms of physical fidelity, how different is the INSTRUMENTATION in the TTC simulator from the control room at your assigned plant? Example differences may include the look, sound, layout, location, and existence of INSTRUMENTATION. * (show/hide trigger question)

\begin{tabular}{|l|l|l|l|l|l|l|}
\hline & 1 & 2 & 3 & 4 & 5 & Entirely Different \\
\hline
\end{tabular}

37. What impact did these differences in INSTRUMENTATION have on your ability to perform your job duties when you first arrived at your assigned plant after your initial TTC simulator training? *

\begin{tabular}{|c|c|c|c|c|c|c|}
\hline & 1 & 2 & 3 & 4 & 5 & High Impact \\
\hline
\end{tabular}

38. Please describe the differences in INSTRUMENTATION that had the most significant effect on your ability to perform your job duties after your initial simulator training:

[Space for response text]

\section{Effect of Differences in Alarms/Annunciators (Non-refresher)}

Please consider the TTC simulator training you have received associated with your current job position at your currently assigned plant only.

39. In terms of physical fidelity, how different are the ALARMSIANNUNCIATORS in the TTC simulator from the control room at your assigned plant? Example differences may include the look, sound, layout, location, equipment/presentation medium, and existence of ALARMS/ANNUNCIATORS. * (show/hide trigger question)

\begin{tabular}{|l|l|l|l|l|l|l|}
\hline & 1 & 2 & 3 & 4 & 5 & Entirely Different \\
\hline
\end{tabular}

40. What impact did these differences in ALARMS/ANNUNCIATORS have on your ability to perform your job duties when you first arrived at your assigned plant after your initial TTC simulator training? *

\begin{tabular}{|c|c|c|c|c|c|c|}
\hline & 1 & 2 & 3 & 4 & 5 & High Impact \\
\hline
\end{tabular}

41. Please describe the differences in ALARMSIANNUNCIATORS that had the most significant effect on your ability to perform your job duties after your initial simulator training:

[Space for response text] 


\section{Effect of Differences in Displays (Non-refresher)}

Please consider the TTC simulator training you have received associated with your current job position at your currently assigned plant only.

42. In terms of physical fidelity, how different are the COMPUTER DISPLAYS in the TTC simulator from the control room at your assigned plant? Example differences may include the look, layout, location, and existence of COMPUTER DISPLAYS. * (show/hide trigger question)

\begin{tabular}{|l|l|l|l|l|l|l|}
\hline & 1 & 2 & 3 & 4 & 5 & Entirely Different \\
\hline
\end{tabular}

43. What impact did these differences in COMPUTER DISPLAYS have on your ability to perform your job duties when you first arrived at your assigned plant after your initial TTC simulator training? *

\begin{tabular}{|c|c|c|c|c|c|c|}
\hline & 1 & 2 & 3 & 4 & 5 & High Impact \\
\hline
\end{tabular}

44. Please describe the differences in COMPUTER DISPLAYS that had the most significant effect on your ability to perform your job duties after your initial simulator training:

[Space for response text]

\section{Effect of Differences in Procedures (Non-refresher)}

Please consider the TTC simulator training you have received associated with your current job position at your currently assigned plant only.

45. In terms of physical fidelity, how different are the PROCEDURES in the TTC simulator from the control room at your assigned plant? Example differences may include the look, layout/organization, storage locations, and equipment/presentation medium of PROCEDURES. Please do not consider differences in Procedure content. * (show/hide trigger question)

\begin{tabular}{|l|l|l|l|l|l|l|}
\hline & 1 & 2 & 3 & 4 & 5 & \\
No Difference & $\mathrm{O}$ & $\mathrm{O}$ & $\mathrm{O}$ & $\mathrm{O}$ & $\mathrm{O}$ & Entirely Different \\
\hline
\end{tabular}

46. What impact did these differences in PROCEDURES have on your ability to perform your job duties when you first arrived at at your assigned plant after your initial TTC simulator training? *

\begin{tabular}{|c|c|c|c|c|c|c|}
\hline & 1 & 2 & 3 & 4 & 5 & High Impact \\
\hline
\end{tabular}

47. Please describe the differences in PROCEDURES that had the most significant effect on your ability to perform your job duties after your initial simulator training. Please do not consider differences in Procedure content.

[Space for response text] 


\section{Effect of Differences in Control Room Environment (Non-refresher)}

Please consider the TTC simulator training you have received associated with your current job position at your currently assigned plant only.

48. In terms of physical fidelity, how different is the CONTROL ROOM ENVIRONMENT in the TTC simulator from the control room at your assigned plant? Example differences may include the look, lighting, sound/ambient noise, and layout of the CONTROL ROOM ENVIRONMENT. * (show/hide trigger question)

\begin{tabular}{|l|l|l|l|l|l|l|}
\hline & 1 & 2 & 3 & 4 & 5 & Entirely Different \\
\hline
\end{tabular}

49. What impact did these differences in the CONTROL ROOM ENVIRONMENT have on your ability to perform your job duties when you first arrived at your assigned plant after your initial TTC simulator training? *

\begin{tabular}{|c|c|c|c|c|c|c|}
\hline & 1 & 2 & 3 & 4 & 5 & High Impact \\
\hline
\end{tabular}

50. Please describe the differences in CONTROL ROOM ENVIRONMENT that had the most significant effect on your ability to perform your job duties after your initial simulator training:

[Space for response text]

\section{Differences Training}

Please consider the TTC simulator training you have received associated with your current job position at your currently assigned plant only.

51. In the TTC simulator training classes you received which methods were used to address the potential physical fidelity differences between the TTC simulator on which you were trained and the control room at your assigned plant. *

\begin{tabular}{|l|c|c|c|c|}
\hline & $\begin{array}{l}\text { Differences were } \\
\text { neither explained } \\
\text { nor pointed out }\end{array}$ & $\begin{array}{l}\text { Differences were } \\
\text { pointed out } \\
\text { without detail }\end{array}$ & $\begin{array}{l}\text { Differences were } \\
\text { explained in some } \\
\text { detail }\end{array}$ & $\begin{array}{l}\text { Differences were } \\
\text { explained in great } \\
\text { detail }\end{array}$ \\
\hline $\begin{array}{l}\text { Instructor } \\
\text { addressed } \\
\text { differences }\end{array}$ & $\mathrm{O}$ & $\mathrm{O}$ & 0 & $\mathrm{O}$ \\
\hline $\begin{array}{l}\text { Training materials } \\
\text { and/or } \\
\text { handouts } \\
\text { addressed } \\
\text { differences }\end{array}$ & $\mathrm{O}$ & $\mathrm{O}$ & $\mathrm{O}$ & $\mathrm{O}$ \\
\hline $\begin{array}{l}\text { Students } \\
\text { discussed } \\
\text { differences } \\
\text { among } \\
\text { themselves }\end{array}$ & $\mathrm{O}$ & $\mathrm{O}$ & 0 & 0 \\
\hline
\end{tabular}


52. Once at your assigned plant what did you have to do, if anything, to deal with the differences?

[Space for response text]

\section{Background}

53. Years in Resident Inspector position (round to nearest year):

54. Type of reactor(s) associated with current assignment: *

Yes No

Babcock and Wilcox

Combustion Engineering

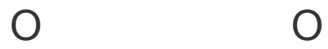

General Electric

Westinghouse

55. Please select the job positions for which you have previous experience:

Have previous experience?

Yes No

Operator Licensing Examiner

Years

$\mathrm{O}$

O

Shift Technical Advisor

$0 \quad 0$

Reactor Operator

O

O

Senior Reactor Operator

O

O

Shift Supervisor

O

O

56. Please list other experience. Other experience may include assignments in the nuclear navy, as a nuclear instructor/trainer, or any other relevant experience.

Position Years

1.

2.

3.

4.

5. 


\section{TTC Training Experience}

57. Types of TTC System (classroom) Training Received

Received

Yes No

Babcock and Wilcox

$0 \quad 0$

Combustion Engineering

$\mathrm{O}$

$\mathrm{O}$

General Electric

$\mathrm{O}$

$\mathrm{O}$

Westinghouse

$\mathrm{O}$

$\mathrm{O}$

58. Types of TTC Simulator Training Received

\section{Received Simulator Refresher \\ Courses Attended \\ Yes No}

Babcock and Wilcox

Combustion Engineering

General Electric

Westinghouse

\section{Number of TTC}

$\mathrm{O}$

$\mathrm{O}$

$\mathrm{O}$

$\mathrm{O}$

59. Years since your last TTC simulator training class (round to nearest year; if less than 6 months, respond "0")? *

\section{Comments}

60. Please add any comments you have on the physical fidelity of simulators for training resident inspectors:

[Space for response text] 


\section{TTC Simulator Training Appraisal}

The following questions seek your appraisal of your TTC simulator training. Response should appraise your TTC simulator training separately from (a) any simulator training not provided at the TTC, (b) individual studies, (c) classroom or computer-delivered courses, or (d) on-the-job-training.

Please use the following scale to appraise how the simulator training you received at the TTC contributed to your success in performing your job responsibilities.

Simulator training was:

Counter-productive (i.e., negative impact on my performance and readiness for off-normal events)

Unnecessary (i.e., had no impact on my performance and readiness)

Positive impact, but too little value (i.e., had some positive impact, but not worth my time/effort)

Valuable (i.e., had enough positive impact to be worth my time/effort)

Essential (i.e., could not have achieved successful job performance and readiness without it)

\section{How necessary was your TTC simulator training for:}

61. Developing your knowledge of nuclear facility operations including system performance and interrelationships. * (show/hide trigger question)

\begin{tabular}{|c|c|c|c|c|}
\hline Counter-productive & Unnecessary & $\begin{array}{c}\text { Positive impact, but } \\
\text { too little value }\end{array}$ & Valuable & Essential \\
\hline $\mathrm{O}$ & $\mathrm{O}$ & $\mathrm{O}$ & $\mathrm{O}$ & $\mathrm{O}$ \\
\hline
\end{tabular}

62. [Skip link] Why was your TTC simulator training counter-productive for developing your knowledge of nuclear facility operations?

[Space for response text]

63. [Skip link] Why was your TTC simulator training unnecessary for developing your knowledge of operators' roles and responsibilities in the control room during both normal operations and off-normal events?

I already knew this before the TTC simulator training.

Other training was sufficient.

Other (please state):

64. Developing your knowledge of operators' roles and responsibilities in the control room during both normal operations and off-normal events. * (show/hide trigger question)

\begin{tabular}{|c|c|c|c|c|}
\hline Counter-productive & Unnecessary & $\begin{array}{c}\text { Positive impact, but } \\
\text { too little value }\end{array}$ & Valuable & Essential \\
\hline $\mathrm{O}$ & $\mathrm{O}$ & $\mathrm{O}$ & $\mathrm{O}$ & $\mathrm{O}$ \\
\hline
\end{tabular}


65. [Skip link] Why was your TTC simulator training counter-productive for developing your knowledge of operators' roles and responsibilities in the control room during both normal operations and off-normal events?

[Space for response text]

66. [Skip link] Why was your TTC simulator Training unnecessary for developing your knowledge of nuclear facility operations including system performance and interrelationships?

I already knew this before the TTC simulator training.

Other training was sufficient.

Other (please state):

67. Developing your regulatory skills for administering, documenting, and justifying a licensing evaluation of operator and crew performance in the control room during normal and off-normal plant operation. * (show/hide trigger question)

\begin{tabular}{|c|c|c|c|c|}
\hline Counter-productive & Unnecessary & $\begin{array}{c}\text { Positive impact, but } \\
\text { too little value }\end{array}$ & Valuable & Essential \\
\hline $\mathrm{O}$ & $\mathrm{O}$ & $\mathrm{O}$ & $\mathrm{O}$ & $\mathrm{O}$ \\
\hline
\end{tabular}

68. [Skip link] Why was your TTC simulator training counter-productive for developing your regulatory skills for administering, documenting, and justifying a licensing evaluation of operator and crew performance?

[Space for response text]

69. [Skip link] Why was your TTC simulator training unnecessary for developing your regulatory skills for administering, documenting, and justifying a licensing evaluation of operator and crew performance?

I already knew this before the TTC simulator training.

Other training was sufficient.

Other (please state):

70. How might the TTC simulator training be changed so that it is more beneficial to your success in performing your job responsibilities?

[Space for response text] 


\title{
23. Contact
}

71. Would you be willing to discuss your responses with project staff or participate in a focus group of your peers and TTC training instructors? *
O Yes
O No

\begin{abstract}
A Note on Privacy
Unless you voluntarily provide your name or telephone number for follow up questions by project staff or potential participation in a focus group, this survey is anonymous and the record kept of your survey responses does not contain any identifying information about you. Aggregated survey results may be shared publicly. Such aggregated results will contain no personally identifying information.
\end{abstract}

[Skip link] Please provide your contact information:

72. Name:

73. Phone number:

74. Email address: 


\section{Familiarity with New Control Room Technology}

75. In the context of reactor control rooms only, please rate your familiarity with the human-systems interface technology proposed for new reactor control rooms: *

\begin{tabular}{|c|c|c|c|c|c|}
\hline & $\begin{array}{l}\text { Unfa- } \\
\text { miliar } \\
1 \\
1\end{array}$ & 2 & 3 & 4 & $\begin{array}{l}\text { Very } \\
\text { Familiar } \\
5 \\
5\end{array}$ \\
\hline $\begin{array}{l}\text { Graphical User Interfaces (displays using } \\
\text { graphical elements such as icons, menus, and } \\
\text { windows which operators interact with using } \\
\text { pointing devices such as a mouse, trackball, or } \\
\text { touchscreen) }\end{array}$ & $\mathrm{O}$ & $\mathrm{O}$ & $\mathrm{O}$ & 0 & $\mathrm{O}$ \\
\hline $\begin{array}{l}\text { Group-view display systems using large panel } \\
\text { computer displays that allow multiple personnel } \\
\text { to view the same information simultaneously. }\end{array}$ & 0 & $\mathrm{O}$ & $\mathrm{O}$ & 0 & $\mathrm{O}$ \\
\hline $\begin{array}{l}\text { Soft Control Systems (control interfaces are in } \\
\text { software and are not hardware knobs, buttons, } \\
\text { switches, etc.) }\end{array}$ & $\mathrm{O}$ & $\mathrm{O}$ & $\mathrm{O}$ & 0 & $\mathrm{O}$ \\
\hline $\begin{array}{l}\text { Computer-based Procedure Systems } \\
\text { (procedures are presented and monitored on a } \\
\text { computer display) }\end{array}$ & $\mathrm{O}$ & 0 & 0 & 0 & 0 \\
\hline $\begin{array}{l}\text { Computerized Operator Support Systems } \\
\text { (computer technology used to support operator } \\
\text { performance in activities such as situation } \\
\text { assessment, fault detection and diagnosis, } \\
\text { safety function and plant performance } \\
\text { monitoring, and response planning) }\end{array}$ & $\mathrm{O}$ & $\mathrm{O}$ & $\mathrm{O}$ & 0 & 0 \\
\hline $\begin{array}{l}\text { Electronic messaging (email, instant } \\
\text { messaging, electronic discussion }\end{array}$ & 0 & $\mathrm{O}$ & 0 & 0 & $\mathrm{O}$ \\
\hline
\end{tabular}

\section{Thank You'/Redirect Page}

Thank you for completing the survey. Your response is very important to us.

For more information on this project please contact:

Mark Miller Chief, Technical Training Support Technical Training Center NRC

(423) 855-6507 


\section{Appendix E.2 On-line Survey: Operator Licensing Examiners \\ Reformatted for print version}

In preparation for training NRC inspectors and license examiners for next generation reactors (e.g., AP-1000, ABWR, EPR, and ESBWR) the NRC has commissioned Pacific Northwest National Laboratory to characterize TTC simulator needs, especially with regard to physical fidelity. Physical fidelity is how closely the look, feel, sound, layout, location, and operator interface of a training simulator matches an actual control room or site-specific control room simulator. This questionnaire seeks your expert input on your experience regarding:

- The most significant job-related differences in physical fidelity between a TTC simulator you trained on and a site-specific control room simulator on which you have recently performed examinations.

- How these differences affected your learning the necessary job-related skills and knowledge during TTC simulator training.

- How these differences affected your ability to apply or transfer your TTC simulator training to the sitespecific simulator.

- How your training addressed these differences.

This questionnaire also seeks your assessment of the TTC simulator training you received.

This questionnaire is specifically for Operator Licensing Examiners only. If you are not an Operator Licensing Examiner, please do not complete this questionnaire.

${ }^{*}$ Questions with a red asterisk are required.

A Note on Privacy

Unless you voluntarily provide your name or telephone number for follow up questions by project staff or potential participation in a focus group, this survey is anonymous and the record kept of your survey responses does not contain any identifying information about you. Aggregated survey results may be shared publicly. Such aggregated results will contain no personally identifying information. 


\section{Effect of Differences in Physical Fidelity}

For each major control room attribute listed you are asked to assess the effect of physical fidelity differences between a TTC training simulator and a site-specific control room simulator of a plant at which you have recently performed examinations and to characterize the most significant job-related differences.

You will be assessing the following control room simulator attributes:

- Panels

- Controllers and Switches

- Instrumentation

- Alarms/Annunciators

- Computer Displays

- Procedures

- Control Room Environment

\section{Special Instructions for License Examiners}

\section{IMPORTANT!}

The following questions will ask you to assess differences between a site-specific simulator and a TTC simulator. Your assessments should consider a single site-specific simulator that you have recently performed examinations on. Please use only the same site-specific simulator and the TTC simulator for the same-vendor reactor to answer ALL questions that ask about differences.

\section{Refresher Training}

Please consider the TTC simulator training you have received associated with your current job position only. Your assessments should consider a single site-specific simulator that you have recently performed examinations on. Please use only the same site-specific simulator and the TTC simulator for the same-vendor reactor to answer ALL questions that ask about differences.

1. Have you received refresher training on the TTC simulator of the same-vendor reactor as the site-specific control room simulator you have recently performed examinations on and that you will use for your assessments in this questionnaire? * (simple page jumping)

O Yes; O No 


\section{Effect of Differences in Panels (Refresher)}

Please consider the TTC simulator training you have received associated with your current job position only. Your assessments should consider a single site-specific simulator that you have recently performed examinations on. Please use only the same site-specific simulator and the TTC simulator for the same-vendor reactor to answer ALL questions that ask about differences.

2. In terms of physical fidelity, how different are the PANELS in the TTC simulator from the site-specific simulator? Example differences may include the layout, location, and existence of PANELS. *

\begin{tabular}{|l|l|l|l|l|l|l|}
\hline & 1 & 2 & 3 & 4 & 5 & Entirely Different \\
\hline
\end{tabular}

3. What impact did these differences in PANELS have on your ability to perform your job duties when you first arrived at the site-specific simulator after your initial TTC simulator training? *

\begin{tabular}{|c|c|c|c|c|c|c|}
\hline & 1 & 2 & 3 & 4 & 5 & High Impact \\
\hline
\end{tabular}

4. What impact did these differences in PANELS have on how difficult it was for you to learn course lessons during the refresher training? *

\begin{tabular}{|c|c|c|c|c|c|c|}
\hline & 1 & 2 & 3 & 4 & 5 & High Impact \\
\hline
\end{tabular}

5. Please describe the differences in PANELS that had the most significant effect on your ability to perform you job duties after your initial simulator training or learn course lessons during refresher training:

[Space for response text]

\section{Effect of Differences in Controllers/Switches (Refresher)}

Please consider the TTC simulator training you have received associated with your current job position only. Your assessments should consider a single site-specific simulator that you have recently performed examinations on. Please use only the same site-specific simulator and the TTC simulator for the same-vendor reactor to answer ALL questions that ask about differences.

6. In terms of physical fidelity, how different are the CONTROLLER/SWITCHES in the TTC simulator from the sitespecific simulator? Example differences may include the look, feel, layout, location, and existence of CONTROLLER/SWITCHES. *

\begin{tabular}{|l|l|l|l|l|l|l|}
\hline & 1 & 2 & 3 & 4 & 5 & \\
No Difference & $\mathrm{O}$ & $\mathrm{O}$ & $\mathrm{O}$ & $\mathrm{O}$ & $\mathrm{O}$ & Entirely Different \\
\hline
\end{tabular}

7. What impact did these differences in CONTROLLERS/SWITCHES have on your ability to perform your job duties when you first arrived at the site-specific simulator after your initial TTC simulator training? *

\begin{tabular}{|c|c|c|c|c|c|c|}
\hline & 1 & 2 & 3 & 4 & 5 & High Impact \\
\hline
\end{tabular}


8. What impact did these differences in CONTROLLERS/SWITCHES have on how difficult it was for you to learn course lessons during the refresher training? *

\begin{tabular}{|c|l|l|l|l|l|l|}
\hline No Impact & 1 & 2 & 3 & 4 & 5 & High Impact \\
\hline
\end{tabular}

9. Please describe the differences in CONTROLLERS/SWITCHES that had the most significant effect on your ability to perform your job duties after your initial simulator training or learn course lessons during refresher training:

[Space for response text]

\section{Effect of Differences in Instrumentation (Refresher)}

Please consider the TTC simulator training you have received associated with your current job position only. Your assessments should consider a single site-specific simulator that you have recently performed examinations on. Please use only the same site-specific simulator and the TTC simulator for the same-vendor reactor to answer ALL questions that ask about differences.

10. In terms of physical fidelity, how different is the INSTRUMENTATION in the TTC simulator from the site-specific simulator? Example differences may include the look, sound, layout, location, and existence of INSTRUMENTATION. *

\begin{tabular}{|l|l|l|l|l|l|l|}
\hline No Difference & 1 & 2 & 3 & 4 & 5 & Entirely Different \\
\hline
\end{tabular}

11. What impact did these differences in INSTRUMENTATION have on your ability to perform your job duties when you first arrived at the site-specific simulator after your initial TTC simulator training? *

\begin{tabular}{|c|c|c|c|c|c|c|}
\hline No Impact & 1 & 2 & 3 & 4 & 5 & High Impact \\
\hline
\end{tabular}

12. What impact did these differences in INSTRUMENTATION have on how difficult it was for you to learn course lessons during the refresher training? *

\begin{tabular}{|c|l|l|l|l|l|l|}
\hline No Impact & 1 & 2 & 3 & 4 & 5 & High Impact \\
\hline
\end{tabular}

13. Please describe the differences in INSTRUMENTATION that had the most significant effect on your ability to perform your job duties after your initial simulator training or learn course lessons during refresher training:

[Space for response text] 


\section{Effect of Differences in Alarmsl Annunciators (Refresher)}

Please consider the TTC simulator training you have received associated with your current job position only. Your assessments should consider a single site-specific simulator that you have recently performed examinations on. Please use only the same site-specific simulator and the TTC simulator for the same-vendor reactor to answer ALL questions that ask about differences.

14. In terms of physical fidelity, how different is the ALARMS/ ANNUNCIATORS in the TTC simulator from the sitespecific simulator? Example differences may include the look, sound, layout, location, and existence of ALARMS/ ANNUNCIATORS. *

\begin{tabular}{|l|l|l|l|l|l|l|}
\hline & 1 & 2 & 3 & 4 & 5 & \\
No Difference & $\mathrm{O}$ & $\mathrm{O}$ & $\mathrm{O}$ & $\mathrm{O}$ & $\mathrm{O}$ & Entirely Different \\
\hline
\end{tabular}

15. What impact did these differences in ALARMS/ ANNUNCIATORS have on your ability to perform your job duties when you first arrived at the site-specific simulator after your initial TTC simulator training? *

\begin{tabular}{|c|c|c|c|c|c|c|}
\hline & 1 & 2 & 3 & 4 & 5 & High Impact \\
\hline
\end{tabular}

16. What impact did these differences in ALARMS/ ANNUNCIATORS have on how difficult it was for you to learn course lessons during the refresher training? *

\begin{tabular}{|c|c|c|c|c|c|c|}
\hline & 1 & 2 & 3 & 4 & 5 & High Impact \\
\hline
\end{tabular}

17. Please describe the differences in ALARMSI ANNUNCIATORS that had the most significant effect on your ability to perform your job duties after your initial simulator training or learn course lessons during refresher training:

[Space for response text]

\section{Effect of Differences in Displays (Refresher)}

Please consider the TTC simulator training you have received associated with your current job position only. Your assessments should consider a single site-specific simulator that you have recently performed examinations on. Please use only the same site-specific simulator and the TTC simulator for the same-vendor reactor to answer ALL questions that ask about differences.

18. In terms of physical fidelity, how different is the COMPUTER DISPLAYS in the TTC simulator from the sitespecific simulator? Example differences may include the look, sound, layout, location, and existence of COMPUTER DISPLAYS. *

\begin{tabular}{|l|l|l|l|l|l|l|}
\hline & 1 & 2 & 3 & 4 & 5 & Entirely Different \\
\hline
\end{tabular}

19. What impact did these differences in COMPUTER DISPLAYS have on your ability to perform your job duties when you first arrived at the site-specific simulator after your initial TTC simulator training? *

\begin{tabular}{|c|c|c|c|c|c|c|}
\hline & 1 & 2 & 3 & 4 & 5 & High Impact \\
\hline
\end{tabular}


20. What impact did these differences in COMPUTER DISPLAYS have on how difficult it was for you to learn course lessons during the refresher training? *

\begin{tabular}{|c|c|c|c|c|c|c|}
\hline & 1 & 2 & 3 & 4 & 5 & High Impact \\
\hline
\end{tabular}

21. Please describe the differences in COMPUTER DISPLAYS that had the most significant effect on your ability to perform your job duties after your initial simulator training or learn course lessons during refresher training:

[Space for response text]

\section{Effect of Differences in Procedures (Refresher)}

Please consider the TTC simulator training you have received associated with your current job position only. Your assessments should consider a single site-specific simulator that you have recently performed examinations on. Please use only the same site-specific simulator and the TTC simulator for the same-vendor reactor to answer ALL questions that ask about differences.

22. In terms of physical fidelity, how different is the PROCEDURES in the TTC simulator from the site-specific simulator? Example differences may include the look, sound, layout, location, and existence of PROCEDURES. *

\begin{tabular}{|l|l|l|l|l|l|l|}
\hline & 1 & 2 & 3 & 4 & 5 & Entirely Different \\
\hline
\end{tabular}

23. What impact did these differences in PROCEDURES have on your ability to perform your job duties when you first arrived at the site-specific simulator after your initial TTC simulator training? *

\begin{tabular}{|c|c|c|c|c|c|c|}
\hline & 1 & 2 & 3 & 4 & 5 & High Impact \\
\hline
\end{tabular}

24. What impact did these differences in PROCEDURES have on how difficult it was for you to learn course lessons during the refresher training? *

\begin{tabular}{|c|c|c|c|c|c|c|}
\hline & 1 & 2 & 3 & 4 & 5 & High Impact \\
\hline
\end{tabular}

25. Please describe the differences in PROCEDURES that had the most significant effect on your ability to perform your job duties after your initial simulator training or learn course lessons during refresher training:

[Space for response text] 


\section{Effect of Differences in Control Room Environment (Refresher)}

Please consider the TTC simulator training you have received associated with your current job position only. Your assessments should consider a single site-specific simulator that you have recently performed examinations on. Please use only the same site-specific simulator and the TTC simulator for the same-vendor reactor to answer ALL questions that ask about differences.

26. In terms of physical fidelity, how different is the CONTROL ROOM ENVIRONMENT in the TTC simulator from the site-specific simulator? Example differences may include the look, sound, layout, location, and existence of CONTROL ROOM ENVIRONMENT. *

\begin{tabular}{|l|l|l|l|l|l|l|}
\hline & 1 & 2 & 3 & 4 & 5 & \\
No Difference & $\mathrm{O}$ & $\mathrm{O}$ & $\mathrm{O}$ & $\mathrm{O}$ & $\mathrm{O}$ & Entirely Different \\
\hline
\end{tabular}

27. What impact did these differences in CONTROL ROOM ENVIRONMENT have on your ability to perform your job duties when you first arrived at the site-specific simulator after your initial TTC simulator training? *

\begin{tabular}{|c|c|c|c|c|c|c|}
\hline & 1 & 2 & 3 & 4 & 5 & High Impact \\
\hline
\end{tabular}

28. What impact did these differences in CONTROL ROOM ENVIRONMENT have on how difficult it was for you to learn course lessons during the refresher training? *

\begin{tabular}{|c|c|c|c|c|c|c|}
\hline & 1 & 2 & 3 & 4 & 5 & High Impact \\
\hline
\end{tabular}

29. Please describe the differences in CONTROL ROOM ENVIRONMENT that had the most significant effect on your ability to perform your job duties after your initial simulator training or learn course lessons during refresher training:

[Space for response text]

\section{Effect of Differences in Panels (Non-refresher)}

Please consider the TTC simulator training you have received associated with your current job position only. Your assessments should consider a single site-specific simulator that you have recently performed examinations on. Please use only the same site-specific simulator and the TTC simulator for the same-vendor reactor to answer ALL questions that ask about differences.

30. In terms of physical fidelity, how different are the PANELS in the TTC simulator from the site-specific simulator? Example differences may include the look, sound, layout, location, and existence of PANELS. *

\begin{tabular}{|l|l|l|l|l|l|l|}
\hline & 1 & 2 & 3 & 4 & 5 & Entirely Different \\
\hline
\end{tabular}

31. What impact did these differences in PANELS have on your ability to perform your job duties when you first arrived at the site-specific simulator after your initial TTC simulator training? *

\begin{tabular}{|c|c|c|c|c|c|c|}
\hline & 1 & 2 & 3 & 4 & 5 & High Impact \\
\hline
\end{tabular}


32. Please describe the differences in PANELS that had the most significant effect on your ability to perform your job duties after your initial simulator training or learn course lessons during refresher training:

[Space for response text]

\section{Effect of Differences in Controllers/Switches (Non-refresher)}

Please consider the TTC simulator training you have received associated with your current job position only. Your assessments should consider a single site-specific simulator that you have recently performed examinations on.

Please use only the same site-specific simulator and the TTC simulator for the same-vendor reactor to answer ALL questions that ask about differences.

33. In terms of physical fidelity, how different are the CONTROLLER/SWITCHES in the TTC simulator from the sitespecific simulator? Example differences may include the look, feel, layout, location, and existence of CONTROLLER/SWITCHES. *

\begin{tabular}{|l|l|l|l|l|l|l|}
\hline & 1 & 2 & 3 & 4 & 5 & \\
No Difference & 0 & 0 & 0 & 0 & 0 & Entirely Different \\
\hline
\end{tabular}

34. What impact did these differences in CONTROLLERS/SWITCHES have on your ability to perform your job duties when you first arrived at the site-specific simulator after your initial TTC simulator training? *

\begin{tabular}{|c|c|c|c|c|c|c|}
\hline & 1 & 2 & 3 & 4 & 5 & High Impact \\
\hline
\end{tabular}

35. Please describe the differences in CONTROLLERS/SWITCHES that had the most significant effect on your ability to perform your job duties after your initial simulator training or learn course lessons during refresher training:

[Space for response text]

\section{Effect of Differences in Instrumentation (Non-refresher)}

Please consider the TTC simulator training you have received associated with your current job position only. Your assessments should consider a single site-specific simulator that you have recently performed examinations on. Please use only the same site-specific simulator and the TTC simulator for the same-vendor reactor to answer ALL questions that ask about differences.

36. In terms of physical fidelity, how different is the INSTRUMENTATION in the TTC simulator from the site-specific simulator? Example differences may include the look, sound, layout, location, and existence of INSTRUMENTATION. *

\begin{tabular}{|l|l|l|l|l|l|l|}
\hline & 1 & 2 & 3 & 4 & 5 & Entirely Different \\
\hline
\end{tabular}


37. What impact did these differences in INSTRUMENTATION have on your ability to perform your job duties when you first arrived at the site-specific simulator after your initial TTC simulator training? *

\begin{tabular}{|c|c|c|c|c|c|c|}
\hline & 1 & 2 & 3 & 4 & 5 & High Impact \\
\hline
\end{tabular}

38. Please describe the differences in INSTRUMENTATION that had the most significant effect on your ability to perform your job duties after your initial simulator training or learn course lessons during refresher training:

[Space for response text]

\section{Effect of Differences in Alarms/ Annunciators (Non-refresher)}

Please consider the TTC simulator training you have received associated with your current job position only. Your assessments should consider a single site-specific simulator that you have recently performed examinations on. Please use only the same site-specific simulator and the TTC simulator for the same-vendor reactor to answer ALL questions that ask about differences.

39. In terms of physical fidelity, how different is the ALARMS/ ANNUNCIATORS in the TTC simulator from the sitespecific simulator? Example differences may include the look, sound, layout, location, and existence of ALARMS/ ANNUNCIATORS. *

\begin{tabular}{|l|l|l|l|l|l|l|}
\hline & 1 & 2 & 3 & 4 & 5 & Entirely Different \\
\hline
\end{tabular}

40. What impact did these differences in ALARMS/ ANNUNCIATORS have on your ability to perform your job duties when you first arrived at the site-specific simulator after your initial TTC simulator training? *

\begin{tabular}{|c|c|c|c|c|c|c|}
\hline & 1 & 2 & 3 & 4 & 5 & High Impact \\
\hline
\end{tabular}

41. Please describe the differences in ALARMS/ ANNUNCIATORS that had the most significant effect on your ability to perform your job duties after your initial simulator training or learn course lessons during refresher training:

[Space for response text]

\section{Effect of Differences in Displays (Non-refresher)}

Please consider the TTC simulator training you have received associated with your current job position only. Your assessments should consider a single site-specific simulator that you have recently performed examinations on. Please use only the same site-specific simulator and the TTC simulator for the same-vendor reactor to answer ALL questions that ask about differences.

42. In terms of physical fidelity, how different is the COMPUTER DISPLAYS in the TTC simulator from the sitespecific simulator? Example differences may include the look, sound, layout, location, and existence of COMPUTER DISPLAYS. *

\begin{tabular}{|l|l|l|l|l|l|l|}
\hline & 1 & 2 & 3 & 4 & 5 & \\
No Difference & 0 & 0 & 0 & 0 & 0 & Entirely Different \\
\hline
\end{tabular}


43. What impact did these differences in COMPUTER DISPLAYS have on your ability to perform your job duties when you first arrived at the site-specific simulator after your initial TTC simulator training? *

\begin{tabular}{|c|c|c|c|c|c|c|}
\hline & 1 & 2 & 3 & 4 & 5 & High Impact \\
\hline
\end{tabular}

44. Please describe the differences in COMPUTER DISPLAYS that had the most significant effect on your ability to perform your job duties after your initial simulator training or learn course lessons during refresher training:

[Space for response text]

\section{Effect of Differences in Procedures (Non-refresher)}

Please consider the TTC simulator training you have received associated with your current job position only. Your assessments should consider a single site-specific simulator that you have recently performed examinations on. Please use only the same site-specific simulator and the TTC simulator for the same-vendor reactor to answer ALL questions that ask about differences.

45. In terms of physical fidelity, how different is the PROCEDURES in the TTC simulator from the site-specific simulator? Example differences may include the look, sound, layout, location, and existence of PROCEDURES. *

\begin{tabular}{|l|l|l|l|l|l|l|}
\hline & 1 & 2 & 3 & 4 & 5 & Entirely Different \\
\hline
\end{tabular}

46. What impact did these differences in PROCEDURES have on your ability to perform your job duties when you first arrived at the site-specific simulator after your initial TTC simulator training? *

\begin{tabular}{|c|c|c|c|c|c|c|}
\hline & 1 & 2 & 3 & 4 & 5 & High Impact \\
\hline
\end{tabular}

47. Please describe the differences in PROCEDURES that had the most significant effect on your ability to perform your job duties after your initial simulator training or learn course lessons during refresher training:

[Space for response text]

\section{Effect of Differences in Control Room Environment (Non-refresher)}

Please consider the TTC simulator training you have received associated with your current job position only. Your assessments should consider a single site-specific simulator that you have recently performed examinations on. Please use only the same site-specific simulator and the TTC simulator for the same-vendor reactor to answer ALL questions that ask about differences. 
48. In terms of physical fidelity, how different is the CONTROL ROOM ENVIRONMENT in the TTC simulator from the site-specific simulator? Example differences may include the look, sound, layout, location, and existence of CONTROL ROOM ENVIRONMENT. *

\begin{tabular}{|c|c|c|c|c|c|c|}
\hline & 1 & 2 & 3 & 4 & 5 & Entirely Different \\
\hline
\end{tabular}

49. What impact did these differences in CONTROL ROOM ENVIRONMENT have on your ability to perform your job duties when you first arrived at the site-specific simulator after your initial TTC simulator training? *

\begin{tabular}{|c|c|c|c|c|c|c|}
\hline & 1 & 2 & 3 & 4 & 5 & High Impact \\
\hline
\end{tabular}

50. Please describe the differences in CONTROL ROOM ENVIRONMENT that had the most significant effect on your ability to perform your job duties after your initial simulator training or learn course lessons during refresher training:

[Space for response text]

\section{Differences Training}

Please consider the TTC simulator training you have received associated with your current job position only. Your assessments should consider a single site-specific simulator that you have recently performed examinations on. Please use only the same site-specific simulator and the TTC simulator for the same-vendor reactor to answer ALL questions that ask about differences.

51. In the TTC simulator training classes you received which methods were used to address the potential physical fidelity differences between the TTC simulator on which you were trained and the site-specific simulator. *

\begin{tabular}{|l|c|c|c|c|}
\hline & $\begin{array}{l}\text { Differences were } \\
\text { neither explained } \\
\text { nor pointed out }\end{array}$ & $\begin{array}{l}\text { Differences were } \\
\text { pointed out } \\
\text { without detail }\end{array}$ & $\begin{array}{l}\text { Differences were } \\
\text { explained in some } \\
\text { detail }\end{array}$ & $\begin{array}{l}\text { Differences were } \\
\text { explained in great } \\
\text { detail }\end{array}$ \\
\hline $\begin{array}{l}\text { Instructor } \\
\text { addressed } \\
\text { differences }\end{array}$ & 0 & 0 & 0 & 0 \\
\hline $\begin{array}{l}\text { Training materials } \\
\text { and/or } \\
\text { handouts } \\
\text { addressed } \\
\text { differences }\end{array}$ & 0 & 0 & 0 & 0 \\
\hline $\begin{array}{l}\text { Students } \\
\text { discussed } \\
\text { differences } \\
\text { among } \\
\text { themselves }\end{array}$ & 0 & 0 & 0 & 0 \\
\hline
\end{tabular}

52. Once at the site-specific simulator what did you have to do, if anything, to deal with the differences?

[Space for response text] 


\section{Background}

53. Years in License Examiner position (round to nearest year):

54. Type of reactor(s) associated with current assignment: *

Yes No

Babcock and Wilcox

Combustion Engineering

General Electric

Westinghouse

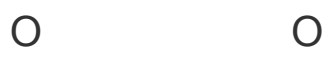

$\mathrm{O} \quad \mathrm{O}$

$\mathrm{O} \quad \mathrm{O}$

$0 \quad 0$

55. Please select the job positions for which you have previous experience:

Have previous experience?

Yes No

Operator Licensing Examiner

Years

$\mathrm{O}$

O

Shift Technical Advisor

$\mathrm{O}$

O

Reactor Operator

O

O

Senior Reactor Operator

O

$\mathrm{O}$

Shift Supervisor

$\mathrm{O}$

$\mathrm{O}$

56. Please list other experience. Other experience may include assignments in the nuclear navy, as a nuclear instructor/trainer, or any other relevant experience.

\section{Position Years}

6.

7.

8.

9.

10. 


\section{TTC Training Experience}

57. Types of TTC System (classroom) Training Received

Received

Yes No

Babcock and Wilcox

$0 \quad 0$

Combustion Engineering

$\mathrm{O}$

$\mathrm{O}$

General Electric

$\mathrm{O}$

$\mathrm{O}$

Westinghouse

$\mathrm{O}$

$\mathrm{O}$

58. Types of TTC Simulator Training Received

\section{Received Simulator Refresher \\ Courses Attended \\ Yes No}

Babcock and Wilcox

Combustion Engineering

General Electric

Westinghouse

\section{Number of TTC}

$\mathrm{O}$

O

$\mathrm{O}$

$\mathrm{O}$

O

O

O

O

59. Years since your last TTC simulator training class (round to nearest year; if less than 6 months, respond "0")? *

\section{Comments}

60. Please add any comments you have on the physical fidelity of simulators for training resident inspectors:

[Space for response text] 


\section{TTC Simulator Training Appraisal}

The following questions seek your appraisal of your TTC simulator training. Response should appraise your TTC simulator training separately from (a) any simulator training not provided at the TTC, (b) individual studies, (c) classroom or computer-delivered courses, or (d) on-the-job-training.

Please use the following scale to appraise how the simulator training you received at the TTC contributed to your success in performing your job responsibilities.

Simulator training was:

Counter-productive (i.e., negative impact on my performance and readiness for off-normal events)

Unnecessary (i.e., had no impact on my performance and readiness)

Positive impact, but too little value (i.e., had some positive impact, but not worth my time/effort)

Valuable (i.e., had enough positive impact to be worth my time/effort)

Essential (i.e., could not have achieved successful job performance and readiness without it)

\section{How necessary was your TTC simulator training for:}

61. Developing your knowledge of nuclear facility operations including system performance and interrelationships. * (show/hide trigger question)

\begin{tabular}{|c|c|c|c|c|}
\hline Counter-productive & Unnecessary & $\begin{array}{c}\text { Positive impact, but } \\
\text { too little value }\end{array}$ & Valuable & Essential \\
\hline $\mathrm{O}$ & $\mathrm{O}$ & $\mathrm{O}$ & $\mathrm{O}$ & $\mathrm{O}$ \\
\hline
\end{tabular}

62. [Skip link] Why was your TTC simulator training counter-productive for developing your knowledge of nuclear facility operations?

[Space for response text]

63. [Skip link] Why was your TTC simulator training unnecessary for developing your knowledge of operators' roles and responsibilities in the control room during both normal operations and off-normal events?

I already knew this before the TTC simulator training.

Other training was sufficient.

Other (please state):

64. Developing your knowledge of operators' roles and responsibilities in the control room during both normal operations and off-normal events. * (show/hide trigger question)

\begin{tabular}{|c|c|c|c|c|}
\hline Counter-productive & Unnecessary & $\begin{array}{c}\text { Positive impact, but } \\
\text { too little value }\end{array}$ & Valuable & Essential \\
\hline $\mathrm{O}$ & $\mathrm{O}$ & $\mathrm{O}$ & $\mathrm{O}$ & $\mathrm{O}$ \\
\hline
\end{tabular}

65. [Skip link] Why was your TTC simulator training counter-productive for developing your knowledge of operators' roles and responsibilities in the control room during both normal operations and off-normal events?

[Space for response text] 
66. [Skip link] Why was your TTC simulator Training unnecessary for developing your knowledge of nuclear facility operations including system performance and interrelationships?

I already knew this before the TTC simulator training.

Other training was sufficient.

Other (please state):

67. Developing your regulatory skills for administering, documenting, and justifying a licensing evaluation of operator and crew performance in the control room during normal and off-normal plant operation. * (show/hide trigger question)

\begin{tabular}{|c|c|c|c|c|}
\hline Counter-productive & Unnecessary & $\begin{array}{c}\text { Positive impact, but } \\
\text { too little value }\end{array}$ & Valuable & Essential \\
\hline $\mathrm{O}$ & $\mathrm{O}$ & $\mathrm{O}$ & $\mathrm{O}$ & $\mathrm{O}$ \\
\hline
\end{tabular}

68. [Skip link] Why was your TTC simulator training counter-productive for developing your regulatory skills for administering, documenting, and justifying a licensing evaluation of operator and crew performance?

[Space for response text]

69. [Skip link] Why was your TTC simulator training unnecessary for developing your regulatory skills for administering, documenting, and justifying a licensing evaluation of operator and crew performance?

I already knew this before the TTC simulator training.

Other training was sufficient.

Other (please state):

71. How might the TTC simulator training be changed so that it is more beneficial to your success in performing your job responsibilities?

[Space for response text] 


\title{
24. Contact
}

71. Would you be willing to discuss your responses with project staff or participate in a focus group of your peers and TTC training instructors? *
O Yes
O No

\begin{abstract}
A Note on Privacy
Unless you voluntarily provide your name or telephone number for follow up questions by project staff or potential participation in a focus group, this survey is anonymous and the record kept of your survey responses does not contain any identifying information about you. Aggregated survey results may be shared publicly. Such aggregated results will contain no personally identifying information.
\end{abstract}

[Skip link] Please provide your contact information:

72. Name:

73. Phone number:

74. Email address: 


\section{Familiarity with New Control Room Technology}

75. In the context of reactor control rooms only, please rate your familiarity with the human-systems interface technology proposed for new reactor control rooms: *

\begin{tabular}{|c|c|c|c|c|c|}
\hline & $\begin{array}{l}\text { Unfa- } \\
\text { miliar } \\
1 \\
1\end{array}$ & 2 & 3 & 4 & $\begin{array}{c}\text { Very } \\
\text { Familiar } \\
5 \\
5\end{array}$ \\
\hline $\begin{array}{l}\text { Graphical User Interfaces (displays using graphical } \\
\text { elements such as icons, menus, and windows } \\
\text { which operators interact with using pointing devices } \\
\text { such as a mouse, trackball, or touchscreen) }\end{array}$ & $\mathrm{O}$ & 0 & 0 & 0 & 0 \\
\hline $\begin{array}{l}\text { Group-view display systems using large panel } \\
\text { computer displays that allow multiple personnel to } \\
\text { view the same information simultaneously. }\end{array}$ & 0 & 0 & 0 & 0 & 0 \\
\hline $\begin{array}{l}\text { Soft Control Systems (control interfaces are in } \\
\text { software and are not hardware knobs, buttons, } \\
\text { switches, etc.) }\end{array}$ & 0 & 0 & 0 & 0 & 0 \\
\hline $\begin{array}{l}\text { Computer-based Procedure Systems (procedures } \\
\text { are presented and monitored on a computer } \\
\text { display) }\end{array}$ & 0 & 0 & 0 & 0 & 0 \\
\hline $\begin{array}{l}\text { Computerized Operator Support Systems } \\
\text { (computer technology used to support operator } \\
\text { performance in activities such as situation } \\
\text { assessment, fault detection and diagnosis, safety } \\
\text { function and plant performance monitoring, and } \\
\text { response planning) }\end{array}$ & 0 & 0 & 0 & $\mathrm{O}$ & 0 \\
\hline $\begin{array}{l}\text { Electronic messaging (email, instant messaging, } \\
\text { electronic discussion }\end{array}$ & 0 & 0 & 0 & 0 & 0 \\
\hline
\end{tabular}

\section{Thank You'/Redirect Page}

Thank you for completing the survey. Your response is very important to us.

For more information on this project please contact:

Mark Miller Chief, Technical Training Support Technical Training Center NRC

(423) 855-6507 


\section{Appendix F}

\section{Draft NRC Advanced Reactor Simulator Fidelity Requirements}




\section{APPENDIX F}

\section{Draft NRC Advanced Reactor Simulator Fidelity Requirements}

Appendix F provides some draft requirements that might be applied to simulators that NRC would obtain for training Resident Inspectors and Operator Licensing Examiners on the advanced Gen III/ Gen III+ reactors. This draft is intended to lay out general requirements for simulator fidelity, not to provide a comprehensive specification.

\subsubsection{Physical Fidelity and Human Factors}

\subsubsection{Scope of Panel Simulation.}

The simulator shall have the capability to represent those panels, consoles, and operating stations required to provide the controls, instrumentation, alarms, and other human-system interfaces necessary for the Nuclear Regulatory Commission (NRC) staff member (hence with referred to as "staff") to become familiar with their design, understand the basis for their use, and have the ability to recognize the correct use by operators in the "reference unit" to conduct the flowing normal evolutions:

(1) Unit startup from cold shutdown to rated power conditions;

(2) Unit shutdown from rated power to cold shutdown conditions; and

(3) Power operations and Load changes.

The simulator shall have the capability to demonstrate the concept of operator conducted surveillance testing on safety-related equipment or systems such that the staff can become familiar with their purpose, understand the basis for their performance, and have the ability to recognize the correct use of the surveillance test procedures by operators in the "reference unit".

Evolutions not listed above, such as reactor core end-of-cycle coastdown, mid-loop operations, refueling operations, evolutions in which the reactor vessel head is removed, may be considered; but are not considered to be within the scope of simulator training.

The determination of the type and number of malfunctions simulated shall be part of a systematic approach to training process for the design of performance-based trainee training programs. The malfunction selection process should utilize the following references:

(1) Industry wide Licensee Event Reports (LERs), Significant Event Reports, and Significant Operating Experience Reports;

(2) Probabilistic risk assessment (PRA) studies;

(3) Nuclear steams supply system and balance of plant manufacturer equipment availability and reliability data, as well as technical information service bulletins;

(4) "Reference unit-specific" operating experiences;

(5) U.S. Nuclear Regulatory Commission bulletins, circulars, and generic letters; and

(6) Reference Unit Safety Analysis Report 
The specific malfunction capability required of the simulator shall meet the requirements specified in the NRC Technical Training Center (TTC) accredited staff training programs.

The simulator shall include, as a minimum, the malfunctions listed below:

(1) Loss of coolant; significant pressurized water reactor (PWR) steam generator tube leaks; inside and outside primary containment; large and small loss of coolant accidents (LOCA) demonstrating multi phase flow; and failure of safety and relief valves;

(2) Loss of instrument air to the extent that the whole system or isolable portions can lose pressure and affect the "reference unit's" static or dynamic performance;

(3) Degraded electrical power to the station, including loss of offsite power, loss of emergency power, loss of emergency generators, loss of power to the unit's electrical distribution buses, and loss of power to the individual instrumentation buses (including AC as well as DC) that provide power to control room instrumentation or unit control functions affecting the unit's response;

(4) Loss of forced core coolant flow due to single or multiple pump failure;

(5) Loss of condenser vacuum, including loss of condenser level control;

(6) Loss of service water or cooling to individual components;

(7) Loss of shutdown cooling;

(8) Loss of component cooling system or cooling to individual components;

(9) Loss of normal feedwater or normal feedwater system failure;

(10) Loss of all feedwater, both normal and emergency;

(11) Loss of a protective system channel;

(12) Control rod failure, including stuck rods, uncoupled rods, drifting rods, rod drops, and misaligned rods;

(13) Inability to drive control rods;

(14) Fuel cladding failure resulting in high activity in reactor coolant or off-gas and the associated high radiation alarms;

(15) Turbine trip;

(16) Generator trip;

(17) Failure in automatic control systems that affect reactivity and core heat removal;

(18) Failure of reactor coolant pressure and volume control systems for PWRs;

(19) Reactor trip;

(20) Main steamline break, as well as main feedline breaks, both inside and outside containment;

(21) Nuclear instrumentation failures;

(22) Process instrumentation, alarms, and control system failures;

(23) Passive failures of components in systems, such as engineered safety features or emergency feedwater systems;

(24) Failure of the automatic reactor trip system; and

(25) Reactor pressure control system failure, including turbine bypass failure for boiling water reactors (BWRs).

The simulator shall support the ability to demonstrate abnormal, off-normal, and emergency events, including simultaneous or sequential malfunctions, for the purpose of the staff member to become familiar with the "reference unit's response and automatic control functions, understand the basis for the response, and have the ability to recognize the correct response by operators in the "reference unit" to conduct the normal and abnormal evolutions list above. Where operator actions vary based on severity of the event, the simulator shall have adjustable malfunction severity of a sufficient range to represent the 
potential "reference unit" conditions. The simulator shall support consequential failures of systems and equipment due to operator action or malfunction of supporting systems where supported by training needs assessment.

The response of the simulator shall be comply with current industry standard compared to actual "reference unit" response or best estimate unit response.

The simulator shall support the ability of the staff to become familiar with the proper operator response, understand the basis for the operator's actions, and have the ability to recognize the correct use of "reference unit" procedures to recover from or mitigate the consequences of malfunctions. The scope of simulation shall be such that a stable, controllable, and safe condition is attained, which can be continued either to cold shutdown conditions or until the limits of simulation are reached.

\subsubsection{Instrumentation, Controls, Markings, and Operator Aids.}

The simulator shall depict panels, consoles, and operating stations shall include instrumentation, controls, markings, operator aids, and other components or displays that are used during normal, abnormal, offnormal, and emergency evolutions in such a manner that staff have the ability to understand the identification, basis, and correct implementation of the following during their training:

- Critical components

- ESF system components

- Critical safety parameters

- Normal operating procedures

- Administrative procedures including tagging, criticality predictions, reactivity balance calculations, temporary change authorizations, jumpers, etc.)

- Annunciator response procedures

- Abnormal operating procedures

- Emergency operating procedures

- Surveillance and test procedures

- Technical Specification

The following items or facsimiles shall be considered:

- Switches

- Controllers

- Meters

- Recorders

- Mimics

- Demarcation lines

- Engravings

- Color

- Panel layout

- Plant computer

- Lights 
- Annunciators

- Labels

- Tactile cues

- Display systems

The simulator shall support replication and simulation of systems, equipment, individual components, and instrumentation, etc. where supported by a training needs assessment.

\subsubsection{Control Room Environment}

The "reference unit" control room environmental features that support the staff experiencing and becoming familiar with crew interaction during normal, abnormal, off-normal, and emergency evolutions shall be simulated. Communication systems that an operator would typically use to direct remote "reference unit" activities shall be operational at least to the extent that the instructor, when performing these activities, is able to communicate over the appropriate operator's communication system.

The following items shall be considered:

- Crew designated workstations

- Communications

- Audible cues

\subsubsection{4 "Reference Unit" and TTC Simulator (TTCS) Deviations.}

The following terms apply:

- "Reference Unit" - Vendors' typical site specific unit and associated full-scope simulator used for license operator training

- NRC Technical Training Center Simulator (TTCS) - Simulator used to train staff at the NRC Technical Training Center

As a minimum, there shall be a minimum of one (1) TTCS capable of training staff on each thermalhydraulic principal (i.e., pressurized water reactor - PWR and boiling water reactor - BWR. The TTCS shall have the capability to replicate one of the existing vendors" "reference units" per the scope and specifications described in Sections 3.2.1.3. Deviations in physical fidelity and human factors may exist between the "reference units" and the TTCS provided the design and operation of the TTCS meets the training learning objectives defined in the TTC systematic approach to training (SAT) program.

\subsubsection{Systems to be Simulated and the Degree of Completeness}

\subsubsection{Systems Controlled or Monitored from the Control Room.}

The scope of simulation shall include systems of the "reference unit" to the extent necessary to allow the operator to perform the normal evolutions described in 3.2.1.1 and respond to the malfunctions described in 3.2.1.1. These systems shall be complete to the extent that staff can gain an understanding and basis for the control manipulations and observe simulated unit response as if in the "reference unit". The scope of simulation shall include system interactions with other simulated systems to provide a total integrated unit response. 


\subsubsection{Systems Controlled or Monitored External to the Control Room.}

The scope of simulation shall include the systems that are operated or monitored external to the control room that are necessary for staff to gain an understanding and basis for the normal evolutions described in 3.2.1.1 and the expected response to malfunctions described in 3.2.1.1. The operator shall be able to interface with the remote activity utilizing the TTCS communication systems in a manner similar to the "reference unit".

\subsubsection{Physical Fidelity and Human Factors}

\subsubsection{Scope of Panel Simulation.}

The simulator shall include those panels, consoles, and operating stations required to provide the controls, instrumentation, alarms, and other human-system interfaces used by operators in the "reference unit" to conduct the flowing normal evolutions:

(1) Unit startup from cold shutdown to rated power conditions;

(2) Unit shutdown from rated power to cold shutdown conditions;

(3) Power operations and Load changes; and

(4) Operator-conducted surveillance testing on safety-related equipment or systems.

For evolutions not listed above, such as reactor core end-of-cycle coastdown, mid-loop operations, refueling operations, or evolutions in which the reactor vessel head is removed, conditions may be achieved in a non-continuous manner, and mathematical model or initial condition changes are permitted.

The determination of the type and number of malfunctions simulated shall be part of a systematic approach to training process for the design of performance-based operator training programs. The malfunction selection process should utilize the following references:

(1) Licensee Event Reports (LERs), Significant Event Reports, and Significant Operating Experience Reports;

(2) Probabilistic risk assessment (PRA) studies;

(3) Nuclear steams supply system and balance of plant manufacturer equipment availability and reliability data, as well as technical information service bulletins;

(4) Local site considerations and "reference unit-specific" operating experiences;

(5) U.S. Nuclear Regulatory Commission bulletins, circulars, and generic letters; and

(6) Reference Unit Safety Analysis Report

The specific malfunction capability required of the simulator shall meet the requirements specified in the "reference unit's" accredited licensed operator training programs.

The simulator shall include the malfunctions listed below:

(1) Loss of coolant; significant pressurized water reactor (PWR) steam generator tube leaks; inside and outside primary containment; large and small loss of coolant accidents (LOCA) demonstrating multi phase flow; and failure of safety and relief valves;

(2) Loss of instrument air to the extent that the whole system or isolable portions can lose pressure and affect the "reference unit's" static or dynamic performance; 
(3) Degraded electrical power to the station, including loss of offsite power, loss of emergency power, loss of emergency generators, loss of power to the unit's electrical distribution buses, and loss of power to the individual instrumentation buses (including AC as well as DC) that provide power to control room instrumentation or unit control functions affecting the unit's response;

(4) Loss of forced core coolant flow due to single or multiple pump failure;

(5) Loss of condenser vacuum, including loss of condenser level control;

(6) Loss of service water or cooling to individual components;

(7) Loss of shutdown cooling;

(8) Loss of component cooling system or cooling to individual components;

(9) Loss of normal feedwater or normal feedwater system failure;

(10) Loss of all feedwater, both normal and emergency;

(11) Loss of a protective system channel;

(12) Control rod failure, including stuck rods, uncoupled rods, drifting rods, rod drops, and misaligned rods;

(13) Inability to drive control rods;

(14) Fuel cladding failure resulting in high activity in reactor coolant or off-gas and the associated high radiation alarms;

(15) Turbine trip;

(16) Generator trip;

(17) Failure in automatic control systems that affect reactivity and core heat removal;

(18) Failure of reactor coolant pressure and volume control systems for PWRs;

(19) Reactor trip;

(20) Main steamline break, as well as main feedline breaks, both inside and outside containment;

(21) Nuclear instrumentation failures;

(22) Process instrumentation, alarms, and control system failures;

(23) Passive failures of components in systems, such as engineered safety features or emergency feedwater systems;

(24) Failure of the automatic reactor trip system; and

(25) Reactor pressure control system failure, including turbine bypass failure for boiling water reactors (BWRs).

The simulator shall support the conduct of abnormal, off-normal, and emergency events, including simultaneous or sequential malfunctions, to demonstrate inherent "reference unit" response and automatic control functions. Where operator actions vary based on severity of the event, the simulator shall have adjustable malfunction severity of a sufficient range to represent the potential "reference unit" conditions. The simulator shall support consequential failures of systems and equipment due to operator action or malfunction of supporting systems where supposed by a training needs assessment.

The response of the simulator shall be compared to actual "reference unit" response or best estimate unit response. The simulator shall support operator action to recover from or mitigate the consequences of malfunctions. The scope of simulation shall be such that a stable, controllable, and safe condition is attained, which can be continued either to cold shutdown conditions or until the limits of simulation are reached. 


\subsubsection{Instrumentation, Controls, Markings, and Operator Aids.}

[This is from Draft Fidelity Requirements - 1-28-08.doc]The simulator panels, consoles, and operating stations shall include instrumentation, controls, markings, operator aids, and other components or displays that are used during normal, abnormal, off-normal, and emergency evolutions. The following items shall be considered:

- Switches

- Controllers

- Meters

- Recorders

- Mimics

- Demarcation lines

- Engravings

- Color

- Panel layout

- Plant computer

- Lights

- Annunciators

- Labels

- Tactile cues

- Display systems

\subsubsection{Control Room Environment}

The "reference unit" control room environmental features that support normal, abnormal, off-normal, and emergency evolutions shall be simulated. Communication systems that an operator would use to direct remote "reference unit" activities shall be operational at least to the extent that the instructor, when performing these activities, is able to communicate over the appropriate operator's communication system.

The following items shall be considered:

- Floor plan

- Lighting characteristics

- Communications

- Furnishings

- General appearance

- Audible cues

- Obstructions 


\subsubsection{Simulator Control Room Deviations}

Where physical fidelity and human factors deviations exist between the "reference unit" and the simulator, such deviations may remain if a training needs assessment is performed.

\subsubsection{Systems to be Simulated and the Degree of Completeness}

\subsubsection{Systems Controlled or Monitored from the Control Room.}

The scope of simulation shall include systems of the "reference unit" to the extent necessary to allow the operator to perform the normal evolutions described in 3.2.1.1 and respond to the malfunctions described in 3.2.1.1. These systems shall be complete to the extent that the operator can perform these control manipulations and observe simulated unit response as in the "reference unit". The scope of simulation shall include system interactions with other simulated systems to provide a total integrated unit response.

\subsubsection{Systems Controlled or Monitored External to the Control Room.}

The scope of simulation shall include the systems that are operated or monitored external to the control room that are necessary to perform the normal evolutions described in 3.2.1.1 and to respond to malfunctions described in 3.2.1.1. The operator shall be able to interface with the remote activity in the same general way that they would in the actual power plant. 